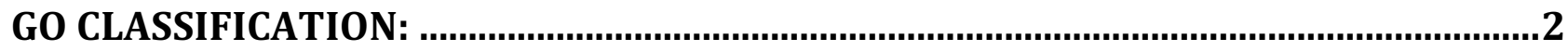

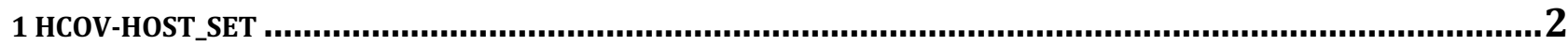

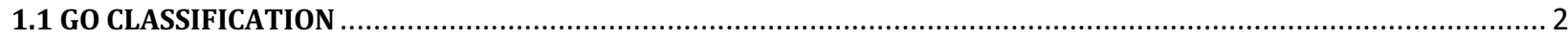

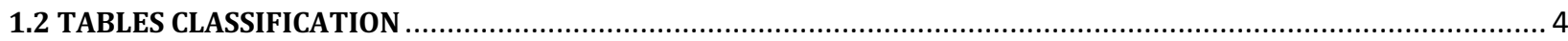

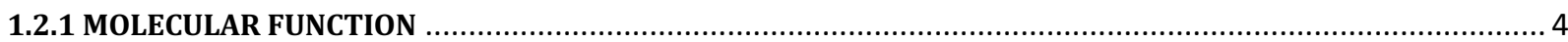

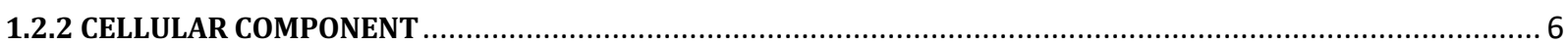

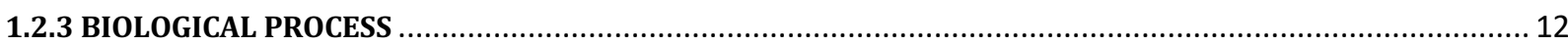

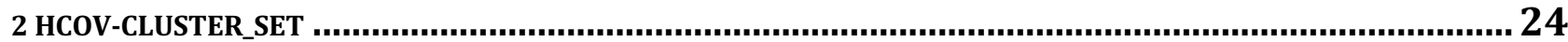

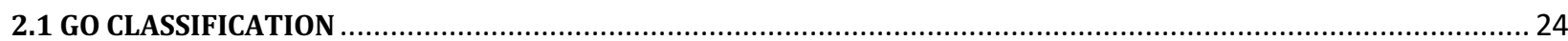

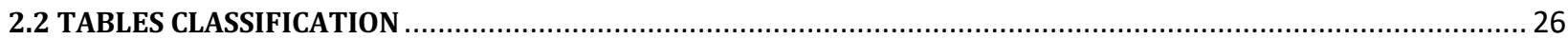

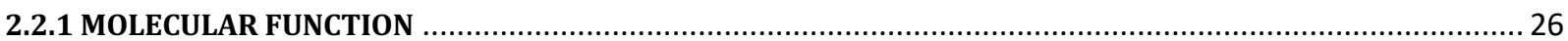

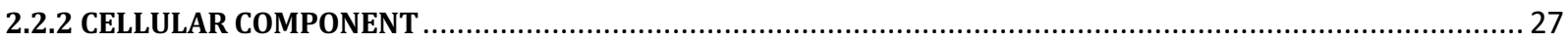

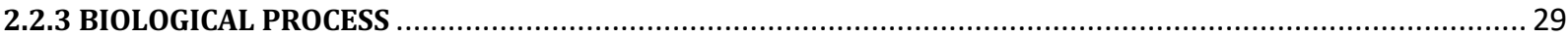

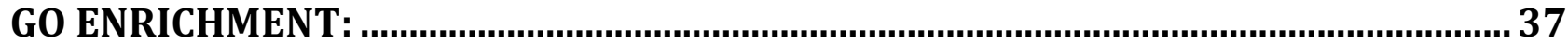

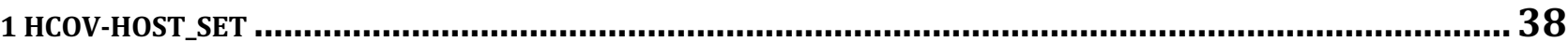

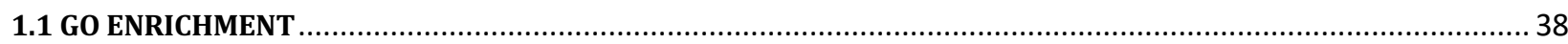

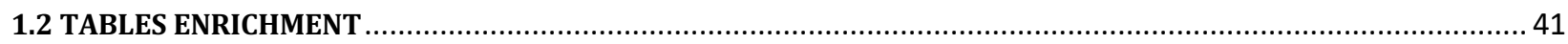

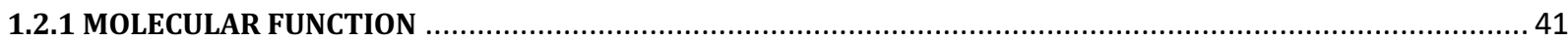

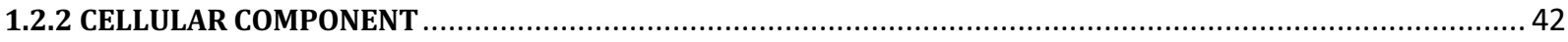

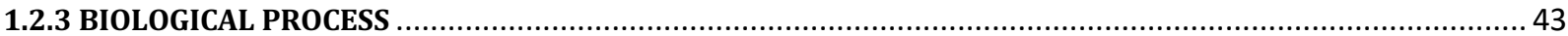

2 HCOV-CLUSTER_SET ........................................................................................................ 54

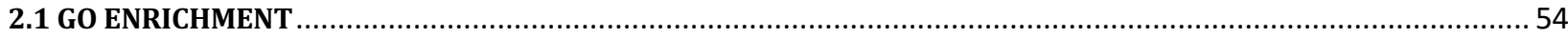

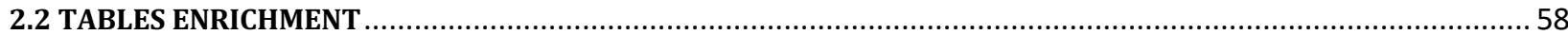

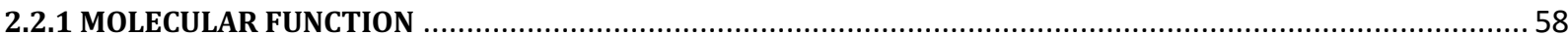

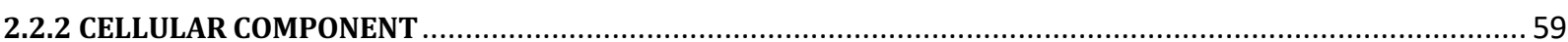

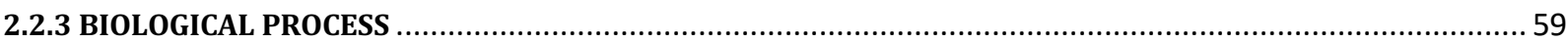

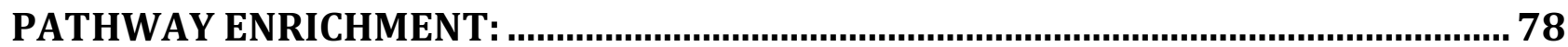

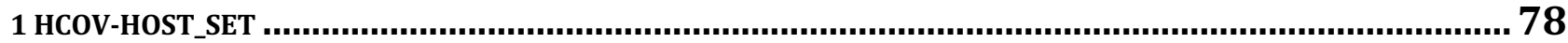

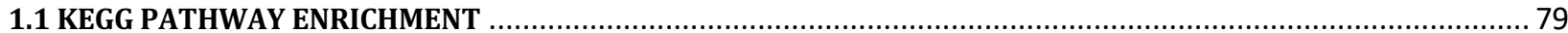

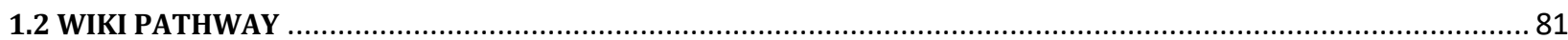

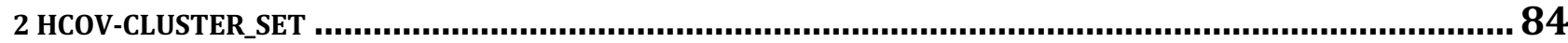

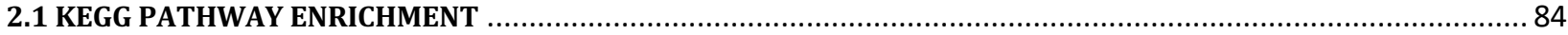

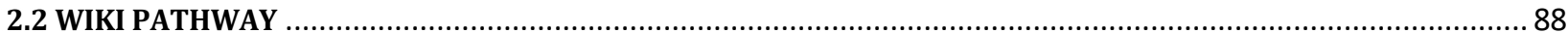

CLUSTER ANALYSIS.............................................................................. 93

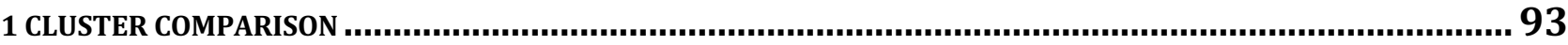

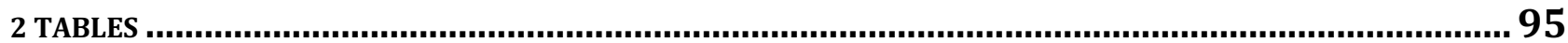

2.1 GO

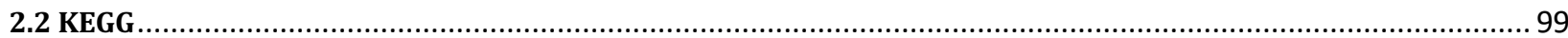

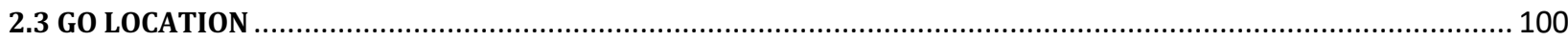




\section{GO CLASSIFICATION:}

\section{HCOV-HOST_SET}

\subsection{GO CLASSIFICATION}

Functional Profile of a gene set at specific GO level. Genes are classified according to their $\mathrm{GO}$ distribution at a specific level.

\section{MOLECULAR FUNCTION}

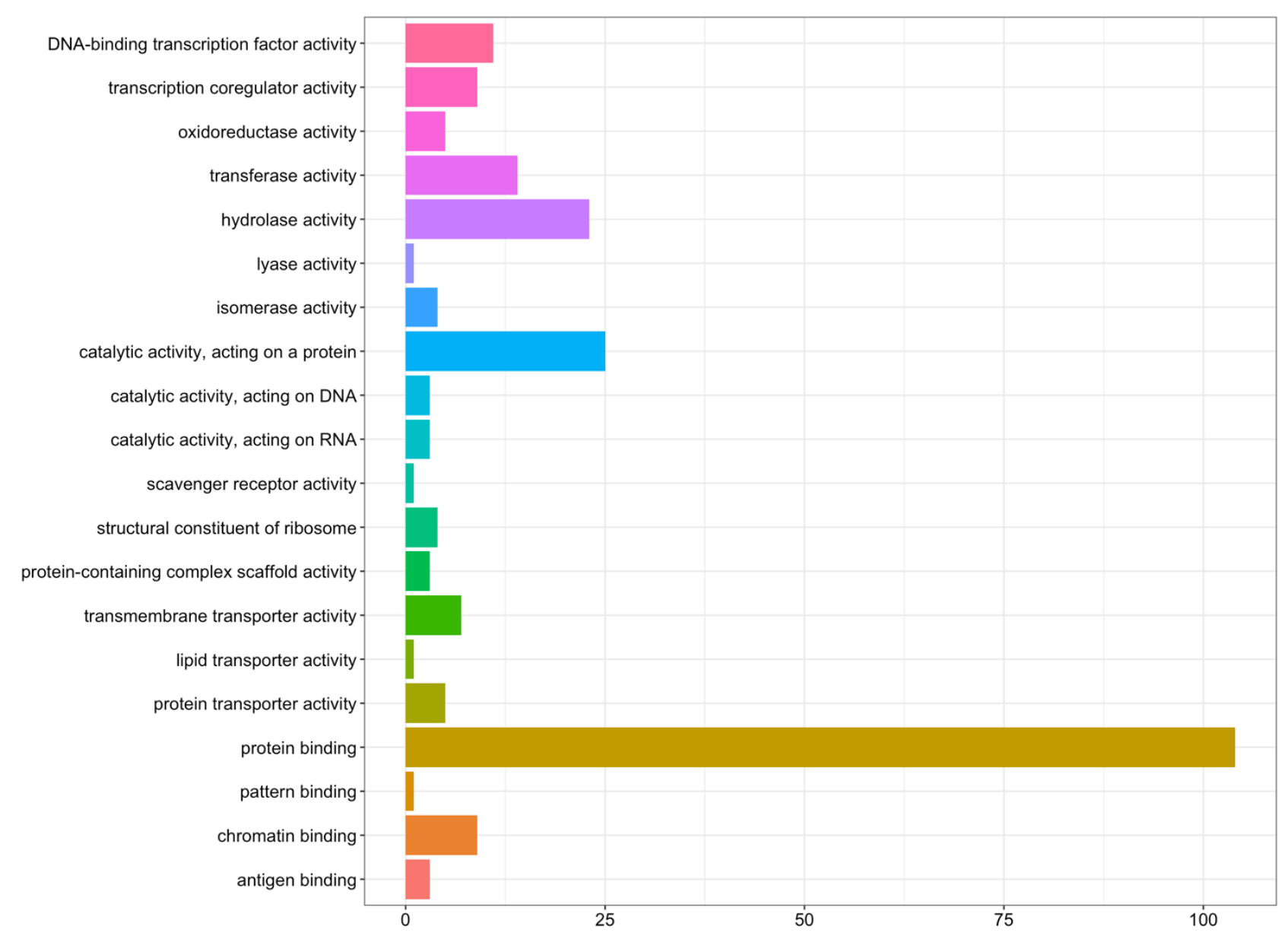




\section{CELLULAR COMPONENT}

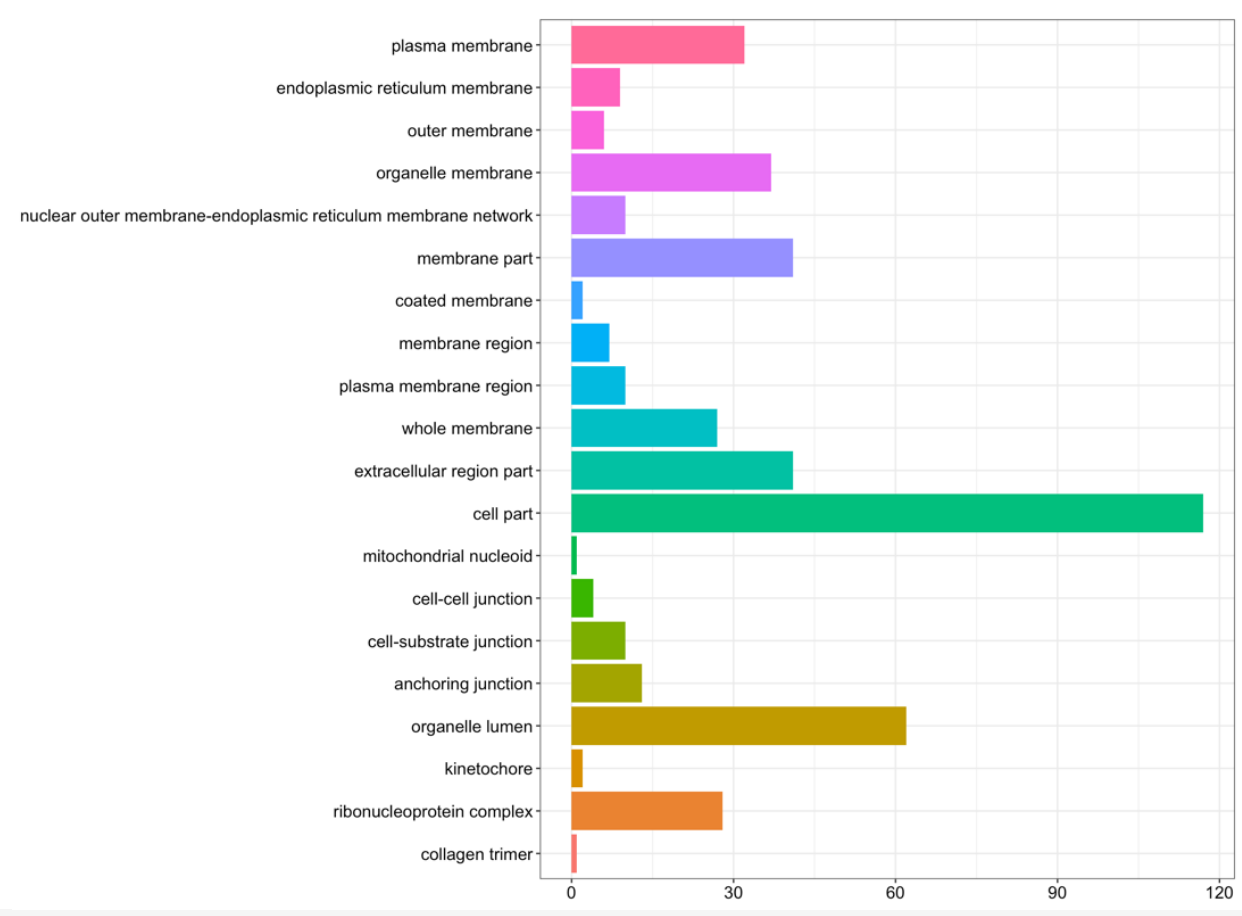

\section{BIOLOGICAL PROCESS}

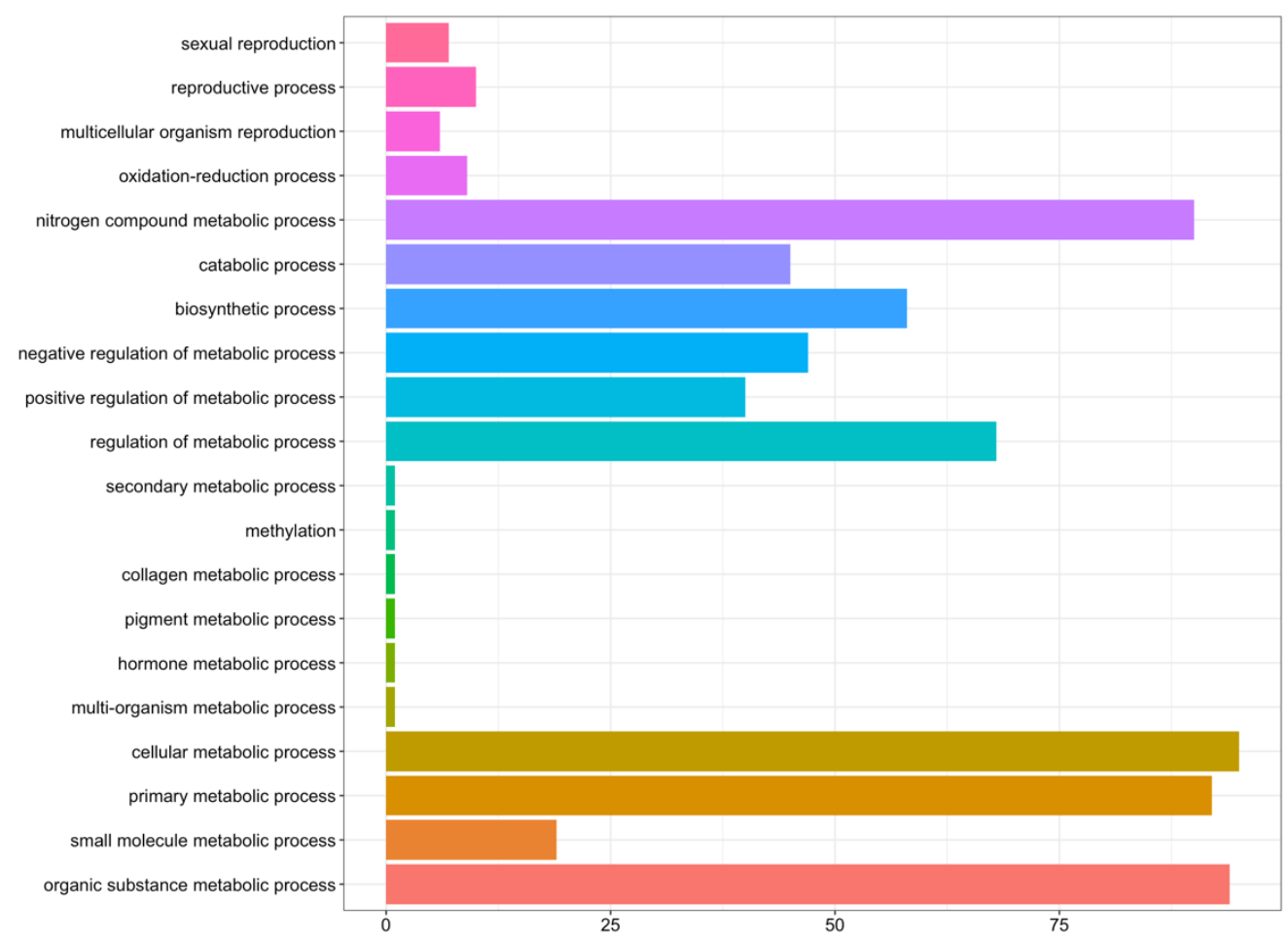




\subsubsection{MOLECULAR FUNCTION}

\begin{tabular}{|c|c|c|c|}
\hline ID & Description & GeneRatio & geneID \\
\hline GO:0003700 & $\begin{array}{l}\text { DNA-binding transcription } \\
\text { factor activity }\end{array}$ & $11 / 119$ & TFEB STAT5A PHB JUN STAT3 SMAD3 ATF5 IRF3 NONO PARP1 TERF1 \\
\hline GO:0003712 & $\begin{array}{l}\text { transcription coregulator } \\
\text { activity }\end{array}$ & $9 / 119$ & JUN SMAD3 ATF5 IRF3 DNAJB1 NPM1 DDX1 RYBP PFDN5 \\
\hline GO:0016491 & oxidoreductase activity & $5 / 119$ & ND4L COX2 RRM2 NDUFA10 VKORC1 \\
\hline GO:0016740 & transferase activity & $14 / 119$ & $\begin{array}{l}\text { YKT6 STAT5A SKP2 IKBKB NMT1 GSK3A GSK3B PARP1 MARK3 MKRN2 MKRN3 } \\
\text { CHEK2 TERF1 UBE2I }\end{array}$ \\
\hline GO:0016787 & hydrolase activity & $23 / 119$ & $\begin{array}{l}\text { PSMC2 PPP1CA FGL2 ACE2 TMPRSS2 ANPEP DPP4 HSPD1 HSPA9 EIF3F KIF11 PSMD1 } \\
\text { SRP54 G3BP1 DDX1 DDAH2 TPSAB1 PSMA2 ABHD17A DCTN2 DDX5 EEF1A1 } \\
\text { ATP6V1G1 }\end{array}$ \\
\hline GO:0016829 & lyase activity & $1 / 119$ & $\mathrm{ACO} 2$ \\
\hline GO:0016853 & isomerase activity & $4 / 119$ & PPIA FKBP1A PPIG PPIH \\
\hline GO:0140096 & $\begin{array}{c}\text { catalytic activity, acting on a } \\
\text { protein }\end{array}$ & $25 / 119$ & $\begin{array}{l}\text { YKT6 STAT5A PPP1CA FGL2 ACE2 PPIA SKP2 TMPRSS2 IKBKB ANPEP DPP4 EIF3F } \\
\text { PSMD1 GSK3A GSK3B PARP1 FKBP1A PPIG MARK3 PPIH TPSAB1 PSMA2 ABHD17A } \\
\text { CHEK2 UBE2I }\end{array}$ \\
\hline GO:0140097 & $\begin{array}{c}\text { catalytic activity, acting on } \\
\text { DNA }\end{array}$ & $3 / 119$ & G3BP1 DDX1 TERF1 \\
\hline GO:0140098 & $\begin{array}{c}\text { catalytic activity, acting on } \\
\text { RNA }\end{array}$ & $3 / 119$ & G3BP1 DDX1 DDX5 \\
\hline GO:0005044 & scavenger receptor activity & $1 / 119$ & TMPRSS2 \\
\hline GO:0003735 & $\begin{array}{l}\text { structural constituent of } \\
\text { ribosome }\end{array}$ & $4 / 119$ & RSL24D1 RPL13A RPL19 RPS20 \\
\hline GO:0032947 & $\begin{array}{l}\text { protein-containing complex } \\
\text { scaffold activity }\end{array}$ & $3 / 119$ & G3BP2 CAV1 SGTA \\
\hline GO:0022857 & $\begin{array}{l}\text { transmembrane transporter } \\
\text { activity }\end{array}$ & $7 / 119$ & COX2 MCL1 BCL2 CEACAM1 ANXA2 HNRNPA3 ATP6V1G1 \\
\hline GO:0005319 & lipid transporter activity & $1 / 119$ & CEACAM1 \\
\hline GO:0008565 & protein transporter activity & $5 / 119$ & KPNB1 XPO1 KPNA2 MCL1 KPNA4 \\
\hline GO:0005515 & protein binding & $104 / 119$ & $\begin{array}{l}\text { BTF3 SCFD1 PSMC2 TFEB TWF2 YKT6 KPNB1 STX5 STAT5A RSL24D1 PHB JUN } \\
\text { STAT3 PPP1CA XPO1 TGFB1 SMAD3 ATF5 COX2 HNRNPA1 ACE2 CLEC4G CD209 } \\
\text { CLEC4M IRF3 KPNA2 SFTPD PPIA BCL2L1 BCL2L2 MCL1 BCL2A1 BCL2 SKP2 KPNA4 } \\
\text { PRKRA CD9 TMPRSS2 IKBKB DPP4 SYNCRIP PTBP1 CEACAM1 ANXA2 HNRNPA2B1 } \\
\text { HNRNPA3 DNAJB1 HSPD1 HSPA9 COPB2 EIF3E EIF3I CHMP4B EIF3F GBF1 RRM2 } \\
\text { KIF11 PSMD1 SRP54 NUDCD1 SNX9 NONO GSK3A GSK3B PABPC1 PABPC4 NPM1 } \\
\text { G3BP1 G3BP2 RPL19 PARP1 NCL DDX1 RYBP FKBP1A PPIG MARK3 PPIH RCAN3 } \\
\text { HGS BAG6 DDAH2 CAMLG CHMP2B SNAP47 MKRN2 TPSAB1 SERPING1 MKRN3 } \\
\text { PSMA2 ABHD17A PFDN5 MIF4GD H2AFY2 RPS20 CHEK2 TERF1 DCTN2 DDX5 } \\
\text { EEF1A1 CAV1 UBE2I SGTA ATP6V1G1 }\end{array}$ \\
\hline GO:0001871 & pattern binding & $1 / 119$ & CLEC4G \\
\hline
\end{tabular}




\begin{tabular}{|c|c|c|c|}
\hline ID & Description & GeneRatio & geneID \\
\hline GO:0003682 & chromatin binding & $9 / 119$ & JUN STAT3 SMAD3 ATF5 NONO NPM1 DDX1 H2AFY2 DDX5 \\
\hline GO:0003823 & antigen binding & $3 / 119$ & TGFB1 CD209 CLEC4M \\
\hline GO:0030246 & carbohydrate binding & $5 / 119$ & CLEC4G CD209 CLEC4M SFTPD NOMO3 \\
\hline GO:0098631 & $\begin{array}{l}\text { cell adhesion mediator } \\
\text { activity }\end{array}$ & $2 / 119$ & PPP1CA ANXA2 \\
\hline GO:0008289 & lipid binding & $8 / 119$ & TWF2 ACBD5 SFTPD ANXA2 HSPD1 GBF1 SNX9 CAV1 \\
\hline GO:0008144 & drug binding & $19 / 119$ & $\begin{array}{l}\text { PSMC2 TWF2 PPIA IKBKB HSPD1 HSPA9 KIF11 SRP54 GSK3A GSK3B G3BP1 DDX1 } \\
\text { FKBP1A PPIG MARK3 PPIH CHEK2 DDX5 UBE2I }\end{array}$ \\
\hline GO:0050840 & extracellular matrix binding & $1 / 119$ & ANXA2 \\
\hline GO:0033218 & amide binding & $11 / 119$ & KPNB1 ACBD5 CD209 CLEC4M KPNA2 PPIA ANPEP HSPD1 SRP54 PPIG PPIH \\
\hline GO:0036094 & small molecule binding & 23/119 & $\begin{array}{l}\text { PSMC2 TWF2 ACBD5 CD209 CLEC4M SFTPD IKBKB HSPD1 HSPA9 KIF11 SRP54 } \\
\text { GSK3A GSK3B G3BP1 PARP1 DDX1 MARK3 DDAH2 CHEK2 DDX5 EEF1A1 CAV1 } \\
\text { UBE2I }\end{array}$ \\
\hline GO:0042562 & hormone binding & $1 / 119$ & HSPD1 \\
\hline GO:0043167 & ion binding & $37 / 119$ & $\begin{array}{c}\text { PSMC2 TWF2 KPNB1 ACBD5 PPP1CA SMAD3 COX2 ACE2 CD209 CLEC4M IKBKB } \\
\text { ANPEP ZCRB1 ANXA2 ACO2 HSPD1 HSPA9 GBF1 RRM2 KIF11 SRP54 SNX9 GSK3A } \\
\text { GSK3B G3BP1 PARP1 DDX1 RYBP MARK3 HGS DDAH2 MKRN2 MKRN3 CHEK2 } \\
\text { DDX5 EEF1A1 UBE2I }\end{array}$ \\
\hline GO:0044877 & $\begin{array}{l}\text { protein-containing complex } \\
\text { binding }\end{array}$ & $15 / 119$ & $\begin{array}{l}\text { SCFD1 TWF2 PPP1CA SMAD3 CD9 HSPD1 KIF11 SRP54 SNX9 NPM1 FKBP1A PPIH } \\
\text { BAG6 DDX5 SGTA }\end{array}$ \\
\hline GO:0046790 & virion binding & $4 / 119$ & CD209 CLEC4M PPIA ANXA2 \\
\hline GO:0048037 & cofactor binding & $4 / 119$ & ACBD5 ACO2 PARP1 VKORC1 \\
\hline GO:0051540 & metal cluster binding & $1 / 119$ & $\mathrm{ACO} 2$ \\
\hline GO:0060090 & molecular adaptor activity & $1 / 119$ & CAV1 \\
\hline GO:0072341 & modified amino acid binding & $1 / 119$ & ANXA2 \\
\hline GO:0097159 & $\begin{array}{l}\text { organic cyclic compound } \\
\text { binding }\end{array}$ & $64 / 119$ & $\begin{array}{l}\text { BTF3 PSMC2 TFEB TWF2 KPNB1 STAT5A RSL24D1 ACBD5 PHB JUN STAT3 XPO1 } \\
\text { SMAD3 ATF5 HNRNPA1 IRF3 KPNA2 PPIA BCL2 PRKRA IKBKB ZCRB1 SYNCRIP } \\
\text { PTBP1 ANXA2 HNRNPA2B1 HNRNPA3 HSPD1 HSPA9 RPL13A EIF3E EIF3I EIF3F } \\
\text { KIF11 SRP54 NACA NONO GSK3A GSK3B PABPC1 PABPC4 NPM1 G3BP1 G3BP2 } \\
\text { RPL19 PARP1 NCL DDX1 RYBP FKBP1A PPIG MARK3 RCAN3 MKRN2 MIF4GD LAS1L } \\
\text { H2AFY2 RPS20 CHEK2 TERF1 DDX5 EEF1A1 CAV1 UBE2I }\end{array}$ \\
\hline GO:0097367 & $\begin{array}{l}\text { carbohydrate derivative } \\
\text { binding }\end{array}$ & $18 / 119$ & $\begin{array}{l}\text { PSMC2 TWF2 ACBD5 SFTPD IKBKB HSPD1 HSPA9 KIF11 SRP54 GSK3A GSK3B G3BP1 } \\
\text { DDX1 MARK3 CHEK2 DDX5 EEF1A1 UBE2I }\end{array}$ \\
\hline GO:1901363 & $\begin{array}{l}\text { heterocyclic compound } \\
\text { binding }\end{array}$ & $63 / 119$ & $\begin{array}{l}\text { BTF3 PSMC2 TFEB TWF2 KPNB1 STAT5A RSL24D1 ACBD5 PHB JUN STAT3 XPO1 } \\
\text { SMAD3 ATF5 HNRNPA1 IRF3 KPNA2 PPIA BCL2 PRKRA IKBKB ZCRB1 SYNCRIP } \\
\text { PTBP1 ANXA2 HNRNPA2B1 HNRNPA3 HSPD1 HSPA9 RPL13A EIF3E EIF3I EIF3F } \\
\text { KIF11 SRP54 NACA NONO GSK3A GSK3B PABPC1 PABPC4 NPM1 G3BP1 G3BP2 } \\
\text { RPL19 PARP1 NCL DDX1 RYBP FKBP1A PPIG MARK3 RCAN3 MKRN2 MIF4GD LAS1L } \\
\text { H2AFY2 RPS20 CHEK2 TERF1 DDX5 EEF1A1 UBE2I }\end{array}$ \\
\hline GO:1901567 & fatty acid derivative binding & $1 / 119$ & ACBD5 \\
\hline GO:1901681 & sulfur compound binding & $1 / 119$ & ACBD5 \\
\hline GO:0005085 & $\begin{array}{l}\text { guanyl-nucleotide exchange } \\
\text { factor activity }\end{array}$ & $1 / 119$ & GBF1 \\
\hline
\end{tabular}




\begin{tabular}{|c|c|c|c|}
\hline ID & Description & GeneRatio & geneID \\
\hline GO:0016247 & channel regulator activity & $2 / 119$ & BCL2 CAV1 \\
\hline GO:0030234 & enzyme regulator activity & $10 / 119$ & JUN TGFB1 PRKRA ANXA2 DNAJB1 PSMD1 NPM1 RCAN3 SERPING1 CAV1 \\
\hline GO:0030545 & receptor regulator activity & $1 / 119$ & TGFB1 \\
\hline GO:0090079 & $\begin{array}{l}\text { translation regulator activity, } \\
\text { nucleic acid binding }\end{array}$ & $2 / 119$ & PABPC1 MIF4GD \\
\hline GO:0038023 & signaling receptor activity & $2 / 119$ & CLEC4M ANPEP \\
\hline GO:0005072 & $\begin{array}{l}\text { transforming growth factor } \\
\text { beta receptor, cytoplasmic } \\
\text { mediator activity }\end{array}$ & $1 / 119$ & SMAD3 \\
\hline GO:0001618 & virus receptor activity & $6 / 119$ & ACE2 CLEC4G CD209 CLEC4M ANPEP DPP4 \\
\hline GO:0140142 & $\begin{array}{l}\text { nucleocytoplasmic carrier } \\
\text { activity }\end{array}$ & $3 / 119$ & XPO1 KPNA2 KPNA4 \\
\hline
\end{tabular}

\subsubsection{CELLULAR COMPONENT}

\begin{tabular}{|c|c|c|c|}
\hline ID & Description & GeneRatio & geneID \\
\hline GO:0005886 & plasma membrane & $32 / 119$ & $\begin{array}{l}\text { SCFD1 YKT6 PHB STAT3 PPP1CA TGFB1 SMAD3 ACE2 CLEC4G CD209 CLEC4M } \\
\text { KPNA2 CD9 TMPRSS2 IKBKB ANPEP DPP4 CEACAM1 ANXA2 HSPD1 NMT1 CHMP4B } \\
\text { SNX9 GSK3B FKBP1A MARK3 CHMP2B SNAP47 ABHD17A EEF1A1 CAV1 ATP6V1G1 }\end{array}$ \\
\hline GO:0005789 & $\begin{array}{l}\text { endoplasmic reticulum } \\
\text { membrane }\end{array}$ & $9 / 119$ & SCFD1 STX5 SFTPD BCL2 COPB2 NOMO3 FKBP1A VKORC1 CAV1 \\
\hline GO:0019867 & outer membrane & $6 / 119$ & PHB BCL2L1 BCL2L2 MCL1 BCL2A1 BCL2 \\
\hline GO:0031090 & organelle membrane & $37 / 119$ & $\begin{array}{l}\text { SCFD1 YKT6 KPNB1 STX5 ACBD5 PHB STAT3 XPO1 SMAD3 ND4L COX2 BCL2L1 } \\
\text { BCL2L2 MCL1 BCL2A1 BCL2 KPNA4 CD9 ANPEP DPP4 CEACAM1 ANXA2 HSPD1 } \\
\text { COPB2 CHMP4B GBF1 SNX9 FKBP1A HGS CHMP2B SNAP47 ABHD17A NDUFA10 } \\
\text { EEF1A1 CAV1 SGTA ATP6V1G1 }\end{array}$ \\
\hline GO:0042175 & $\begin{array}{l}\text { nuclear outer membrane- } \\
\text { endoplasmic reticulum } \\
\text { membrane network }\end{array}$ & $10 / 119$ & SCFD1 STX5 XPO1 SFTPD BCL2 COPB2 NOMO3 FKBP1A VKORC1 CAV1 \\
\hline GO:0044425 & membrane part & $41 / 119$ & $\begin{array}{l}\text { SCFD1 YKT6 STX5 ACBD5 PHB XPO1 ND4L COX2 ACE2 CLEC4G CD209 CLEC4M } \\
\text { KPNA2 SFTPD BCL2L1 MCL1 BCL2 CD9 TMPRSS2 IKBKB ANPEP DPP4 CEACAM1 } \\
\text { ANXA2 HSPD1 COPB2 NMT1 CHMP4B SNX9 NOMO3 FKBP1A CAMLG CHMP2B } \\
\text { SNAP47 ABHD17A NDUFA10 VKORC1 EEF1A1 CAV1 SGTA ATP6V1G1 }\end{array}$ \\
\hline GO:0048475 & coated membrane & $2 / 119$ & COPB2 CHMP4B \\
\hline GO:0098589 & membrane region & $7 / 119$ & XPO1 ACE2 IKBKB DPP4 ANXA2 HSPD1 CAV1 \\
\hline GO:0098590 & plasma membrane region & $10 / 119$ & PHB ACE2 KPNA2 CD9 DPP4 CEACAM1 ANXA2 ABHD17A EEF1A1 CAV1 \\
\hline GO:0098805 & whole membrane & $27 / 119$ & $\begin{array}{c}\text { STX5 ACBD5 PHB XPO1 ACE2 BCL2L1 BCL2L2 MCL1 BCL2A1 BCL2 CD9 IKBKB } \\
\text { ANPEP DPP4 CEACAM1 ANXA2 HSPD1 COPB2 CHMP4B HGS CHMP2B SNAP47 } \\
\text { ABHD17A EEF1A1 CAV1 SGTA ATP6V1G1 }\end{array}$ \\
\hline GO:0044421 & extracellular region part & $41 / 119$ & $\begin{array}{l}\text { TWF2 KPNB1 PHB PPP1CA FGL2 TGFB1 HNRNPA1 ACE2 SFTPD PPIA CD9 TMPRSS2 } \\
\text { ANPEP DPP4 PTBP1 CEACAM1 ANXA2 HNRNPA2B1 DNAJB1 HSPD1 HSPA9 EIF3E }\end{array}$ \\
\hline
\end{tabular}




\begin{tabular}{|c|c|c|c|}
\hline ID & Description & GeneRatio & geneID \\
\hline & & & $\begin{array}{l}\text { EIF3I CHMP4B SNX9 PABPC1 NCL MARK3 HGS BAG6 DDAH2 CHMP2B TPSAB1 } \\
\text { SERPING1 PSMA2 H2AFY2 RPS20 DCTN2 DDX5 EEF1A1 ATP6V1G1 }\end{array}$ \\
\hline GO:0044464 & cell part & $117 / 119$ & $\begin{array}{l}\text { BTF3 SCFD1 PSMC2 TFEB TWF2 YKT6 KPNB1 STX5 STAT5A RSL24D1 ACBD5 PHB } \\
\text { JUN STAT3 PPP1CA SPECC1 FGL2 XPO1 TGFB1 SMAD3 ATF5 ND4L COX2 HNRNPA1 } \\
\text { ACE2 CLEC4G CD209 CLEC4M IRF3 KPNA2 SFTPD PPIA BCL2L1 BCL2L2 MCL1 } \\
\text { BCL2A1 BCL2 SKP2 KPNA4 PRKRA CD9 TMPRSS2 IKBKB ANPEP ZCRB1 DPP4 } \\
\text { SYNCRIP PTBP1 CEACAM1 ANXA2 HNRNPA2B1 HNRNPA3 ACO2 DNAJB1 HSPD1 } \\
\text { HSPA9 COPB2 RPL13A EIF3E EIF3I NMT1 CHMP4B EIF3F GBF1 RRM2 KIF11 PSMD1 } \\
\text { SRP54 NUDCD1 NACA SNX9 NONO GSK3A GSK3B PABPC1 PABPC4 NPM1 G3BP1 } \\
\text { G3BP2 RPL19 PARP1 NCL DDX1 RYBP NOMO3 FKBP1A PPIG MARK3 PPIH RCAN3 } \\
\text { HGS BAG6 DDAH2 CAMLG CHMP2B SNAP47 MKRN2 SERPING1 PSMA2 ABHD17A } \\
\text { PFDN5 MIF4GD NDUFA10 VKORC1 LAS1L H2AFY2 RPS20 CHEK2 TERF1 DCTN2 } \\
\text { DDX5 C11 orf74 EEF1A1 CAV1 UBE2I SGTA ATP6V1G1 }\end{array}$ \\
\hline GO:0042645 & mitochondrial nucleoid & $1 / 119$ & HSPA9 \\
\hline GO:0005911 & cell-cell junction & $4 / 119$ & PPP1CA DPP4 CEACAM1 ANXA2 \\
\hline GO:0030055 & cell-substrate junction & $10 / 119$ & PPIA CD9 DPP4 HSPA9 RPL13A PABPC1 NPM1 G3BP1 RPL19 CAV1 \\
\hline GO:0070161 & anchoring junction & $13 / 119$ & $\begin{array}{l}\text { PPP1CA PPIA CD9 DPP4 CEACAM1 ANXA2 HSPA9 RPL13A PABPC1 NPM1 G3BP1 } \\
\text { RPL19 CAV1 }\end{array}$ \\
\hline GO:0043233 & organelle lumen & $62 / 119$ & $\begin{array}{l}\text { PSMC2 KPNB1 STAT5A RSL24D1 ACBD5 PHB JUN STAT3 PPP1CA FGL2 XPO1 TGFB1 } \\
\text { SMAD3 ATF5 HNRNPA1 IRF3 KPNA2 PPIA BCL2L1 MCL1 BCL2 SKP2 KPNA4 PRKRA } \\
\text { ZCRB1 SYNCRIP PTBP1 ANXA2 HNRNPA2B1 HNRNPA3 ACO2 DNAJB1 HSPD1 HSPA9 } \\
\text { RPL13A EIF3E GBF1 PSMD1 SRP54 NUDCD1 NONO GSK3B NPM1 PARP1 NCL DDX1 } \\
\text { RYBP PPIG PPIH BAG6 SERPING1 PSMA2 MIF4GD NDUFA10 LAS1L H2AFY2 RPS20 } \\
\text { CHEK2 TERF1 DDX5 EEF1A1 UBE2I }\end{array}$ \\
\hline GO:0000776 & kinetochore & $2 / 119$ & XPO1 DCTN2 \\
\hline GO:1990904 & ribonucleoprotein complex & $28 / 119$ & $\begin{array}{l}\text { PSMC2 KPNB1 RSL24D1 XPO1 HNRNPA1 ZCRB1 SYNCRIP HNRNPA2B1 HNRNPA3 } \\
\text { RPL13A EIF3E EIF3I EIF3F SRP54 PABPC1 PABPC4 NPM1 G3BP1 G3BP2 RPL19 NCL } \\
\text { DDX1 PPIH MKRN3 PSMA2 LAS1L RPS20 DDX5 }\end{array}$ \\
\hline GO:0005581 & collagen trimer & $1 / 119$ & SFTPD \\
\hline GO:0005577 & fibrinogen complex & $1 / 119$ & FGL2 \\
\hline GO:0005643 & nuclear pore & $1 / 119$ & KPNB1 \\
\hline GO:0005667 & transcription factor complex & $6 / 119$ & JUN STAT3 SMAD3 ATF5 NONO PARP1 \\
\hline GO:0005838 & $\begin{array}{l}\text { proteasome regulatory } \\
\text { particle }\end{array}$ & $2 / 119$ & PSMC2 PSMD1 \\
\hline GO:0005839 & proteasome core complex & $1 / 119$ & PSMA2 \\
\hline GO:0005852 & $\begin{array}{l}\text { eukaryotic translation } \\
\text { initiation factor } 3 \text { complex }\end{array}$ & $3 / 119$ & EIF3E EIF3I EIF3F \\
\hline GO:0005853 & $\begin{array}{l}\text { eukaryotic translation } \\
\text { elongation factor } 1 \text { complex }\end{array}$ & $1 / 119$ & EEF1A1 \\
\hline GO:0005854 & $\begin{array}{l}\text { nascent polypeptide- } \\
\text { associated complex }\end{array}$ & $1 / 119$ & NACA \\
\hline GO:0005875 & $\begin{array}{l}\text { microtubule associated } \\
\text { complex }\end{array}$ & $2 / 119$ & KIF11 DCTN2 \\
\hline GO:0008540 & $\begin{array}{l}\text { proteasome regulatory } \\
\text { particle, base subcomplex }\end{array}$ & $2 / 119$ & PSMC2 PSMD1 \\
\hline GO:0016272 & prefoldin complex & $1 / 119$ & PFDN5 \\
\hline
\end{tabular}




\begin{tabular}{|c|c|c|c|}
\hline ID & Description & GeneRatio & geneID \\
\hline GO:0017053 & $\begin{array}{l}\text { transcriptional repressor } \\
\text { complex }\end{array}$ & $1 / 119$ & JUN \\
\hline GO:0019773 & $\begin{array}{l}\text { proteasome core complex, } \\
\text { alpha-subunit complex }\end{array}$ & $1 / 119$ & PSMA2 \\
\hline GO:0022624 & $\begin{array}{l}\text { proteasome accessory } \\
\text { complex }\end{array}$ & $2 / 119$ & PSMC2 PSMD1 \\
\hline GO:0030877 & $\begin{array}{l}\text { beta-catenin destruction } \\
\text { complex }\end{array}$ & $2 / 119$ & GSK3A GSK3B \\
\hline GO:0031082 & BLOC complex & $1 / 119$ & SNAP47 \\
\hline GO:0031519 & PcG protein complex & $1 / 119$ & RYBP \\
\hline GO:0031941 & filamentous actin & $1 / 119$ & SPECC1 \\
\hline GO:0032993 & protein-DNA complex & $4 / 119$ & NPM1 PARP1 H2AFY2 TERF1 \\
\hline GO:0036452 & ESCRT complex & $3 / 119$ & CHMP4B HGS CHMP2B \\
\hline GO:0043235 & receptor complex & $4 / 119$ & SMAD3 IKBKB CEACAM1 HSPD1 \\
\hline GO:0044815 & DNA packaging complex & $1 / 119$ & H2AFY2 \\
\hline GO:0070069 & cytochrome complex & $1 / 119$ & $\operatorname{COX} 2$ \\
\hline GO:0070937 & $\begin{array}{l}\text { CRD-mediated mRNA } \\
\text { stability complex }\end{array}$ & $1 / 119$ & SYNCRIP \\
\hline GO:0071141 & SMAD protein complex & $1 / 119$ & SMAD3 \\
\hline GO:0072379 & $\begin{array}{l}\text { ER membrane insertion } \\
\text { complex }\end{array}$ & $2 / 119$ & BAG6 SGTA \\
\hline GO:0072669 & $\begin{array}{l}\text { tRNA-splicing ligase } \\
\text { complex }\end{array}$ & $1 / 119$ & DDX1 \\
\hline GO:0097136 & $\begin{array}{l}\text { Bcl-2 family protein } \\
\text { complex }\end{array}$ & $3 / 119$ & BCL2L1 BCL2L2 MCL1 \\
\hline GO:0097452 & GAIT complex & $2 / 119$ & SYNCRIP RPL13A \\
\hline GO:0098796 & membrane protein complex & $16 / 119$ & $\begin{array}{l}\text { YKT6 STX5 ND4L COX2 BCL2 IKBKB CEACAM1 HSPD1 COPB2 CHMP4B FKBP1A } \\
\text { CHMP2B SNAP47 NDUFA10 CAV1 ATP6V1G1 }\end{array}$ \\
\hline GO:0098798 & $\begin{array}{l}\text { mitochondrial protein } \\
\text { complex }\end{array}$ & $3 / 119$ & ND4L COX2 NDUFA10 \\
\hline GO:0120114 & $\begin{array}{l}\text { Sm-like protein family } \\
\text { complex }\end{array}$ & $1 / 119$ & PPIH \\
\hline GO:1902494 & catalytic complex & $18 / 119$ & $\begin{array}{l}\text { PSMC2 PPP1CA ND4L HNRNPA1 SKP2 PRKRA IKBKB SYNCRIP HNRNPA2B1 } \\
\text { HNRNPA3 PSMD1 PABPC1 PSMA2 NDUFA10 LAS1L DCTN2 DDX5 UBE2I }\end{array}$ \\
\hline GO:1990351 & transporter complex & $1 / 119$ & FKBP1A \\
\hline GO:1990356 & $\begin{array}{l}\text { sumoylated E2 ligase } \\
\text { complex }\end{array}$ & $1 / 119$ & UBE2I \\
\hline GO: 1990667 & PCSK9-AnxA2 complex & $1 / 119$ & ANXA2 \\
\hline
\end{tabular}




\begin{tabular}{|c|c|c|c|}
\hline ID & Description & GeneRatio & geneID \\
\hline GO:1990909 & Wnt signalosome & $1 / 119$ & GSK3B \\
\hline GO:0005929 & cilium & $2 / 119$ & C11orf74 CAV1 \\
\hline GO:0043227 & $\begin{array}{l}\text { membrane-bounded } \\
\text { organelle }\end{array}$ & $107 / 119$ & $\begin{array}{l}\text { BTF3 SCFD1 PSMC2 TFEB TWF2 YKT6 KPNB1 STX5 STAT5A RSL24D1 ACBD5 PHB } \\
\text { JUN STAT3 PPP1CA SPECC1 FGL2 XPO1 TGFB1 SMAD3 ATF5 ND4L COX2 HNRNPA1 } \\
\text { ACE2 IRF3 KPNA2 SFTPD PPIA BCL2L1 BCL2L2 MCL1 BCL2A1 BCL2 SKP2 KPNA4 } \\
\text { PRKRA CD9 TMPRSS2 IKBKB ANPEP ZCRB1 DPP4 SYNCRIP PTBP1 CEACAM1 } \\
\text { ANXA2 HNRNPA2B1 HNRNPA3 ACO2 DNAJB1 HSPD1 HSPA9 COPB2 RPL13A EIF3E } \\
\text { EIF3I NMT1 CHMP4B GBF1 KIF11 PSMD1 SRP54 NUDCD1 NACA SNX9 NONO GSK3A } \\
\text { GSK3B PABPC1 PABPC4 NPM1 G3BP1 PARP1 NCL DDX1 RYBP NOMO3 FKBP1A PPIG } \\
\text { MARK3 PPIH HGS BAG6 DDAH2 CAMLG CHMP2B SNAP47 SERPING1 PSMA2 } \\
\text { ABHD17A PFDN5 MIF4GD NDUFA10 VKORC1 LAS1L H2AFY2 RPS20 CHEK2 TERF1 } \\
\text { DCTN2 DDX5 EEF1A1 CAV1 UBE2I SGTA ATP6V1G1 }\end{array}$ \\
\hline GO:0043228 & $\begin{array}{l}\text { non-membrane-bounded } \\
\text { organelle }\end{array}$ & $47 / 119$ & $\begin{array}{c}\text { PSMC2 TWF2 KPNB1 RSL24D1 JUN STAT3 PPP1CA SPECC1 XPO1 SMAD3 ATF5 } \\
\text { BCL2L1 SKP2 PTBP1 ANXA2 HNRNPA2B1 HNRNPA3 DNAJB1 HSPA9 RPL13A EIF3E } \\
\text { GBF1 KIF11 NONO GSK3B PABPC1 PABPC4 NPM1 G3BP1 RPL19 PARP1 NCL DDX1 } \\
\text { FKBP1A DDAH2 PSMA2 MIF4GD LAS1L H2AFY2 RPS20 CHEK2 TERF1 DCTN2 DDX5 } \\
\text { EEF1A1 CAV1 UBE2I }\end{array}$ \\
\hline GO:0043229 & intracellular organelle & $104 / 119$ & $\begin{array}{l}\text { BTF3 SCFD1 PSMC2 TFEB TWF2 YKT6 KPNB1 STX5 STAT5A RSL24D1 ACBD5 PHB } \\
\text { JUN STAT3 PPP1CA SPECC1 FGL2 XPO1 TGFB1 SMAD3 ATF5 ND4L COX2 HNRNPA1 } \\
\text { IRF3 KPNA2 SFTPD PPIA BCL2L1 BCL2L2 MCL1 BCL2A1 BCL2 SKP2 KPNA4 PRKRA } \\
\text { CD9 IKBKB ANPEP ZCRB1 DPP4 SYNCRIP PTBP1 CEACAM1 ANXA2 HNRNPA2B1 } \\
\text { HNRNPA3 ACO2 DNAJB1 HSPD1 HSPA9 COPB2 RPL13A EIF3E NMT1 CHMP4B GBF1 } \\
\text { KIF11 PSMD1 SRP54 NUDCD1 NACA SNX9 NONO GSK3A GSK3B PABPC1 PABPC4 } \\
\text { NPM1 G3BP1 RPL19 PARP1 NCL DDX1 RYBP NOMO3 FKBP1A PPIG PPIH HGS BAG6 } \\
\text { DDAH2 CAMLG CHMP2B SNAP47 SERPING1 PSMA2 ABHD17A PFDN5 MIF4GD } \\
\text { NDUFA10 VKORC1 LAS1L H2AFY2 RPS20 CHEK2 TERF1 DCTN2 DDX5 EEF1A1 CAV1 } \\
\text { UBE2I SGTA ATP6V1G1 }\end{array}$ \\
\hline GO:0043230 & extracellular organelle & $38 / 119$ & $\begin{array}{c}\text { TWF2 KPNB1 PHB PPP1CA FGL2 HNRNPA1 ACE2 PPIA CD9 TMPRSS2 ANPEP DPP4 } \\
\text { PTBP1 CEACAM1 ANXA2 HNRNPA2B1 DNAJB1 HSPD1 HSPA9 EIF3E EIF3I CHMP4B } \\
\text { SNX9 PABPC1 NCL MARK3 HGS BAG6 DDAH2 CHMP2B SERPING1 PSMA2 H2AFY2 } \\
\text { RPS20 DCTN2 DDX5 EEF1A1 ATP6V1G1 }\end{array}$ \\
\hline GO:0044422 & organelle part & $94 / 119$ & $\begin{array}{l}\text { SCFD1 PSMC2 TWF2 YKT6 KPNB1 STX5 STAT5A RSL24D1 ACBD5 PHB JUN STAT3 } \\
\text { PPP1CA SPECC1 FGL2 XPO1 TGFB1 SMAD3 ATF5 ND4L COX2 HNRNPA1 IRF3 KPNA2 } \\
\text { SFTPD PPIA BCL2L1 BCL2L2 MCL1 BCL2A1 BCL2 SKP2 KPNA4 PRKRA CD9 ANPEP } \\
\text { ZCRB1 DPP4 SYNCRIP PTBP1 CEACAM1 ANXA2 HNRNPA2B1 HNRNPA3 ACO2 } \\
\text { DNAJB1 HSPD1 HSPA9 COPB2 RPL13A EIF3E CHMP4B GBF1 KIF11 PSMD1 SRP54 } \\
\text { NUDCD1 SNX9 NONO GSK3B PABPC1 NPM1 RPL19 PARP1 NCL DDX1 RYBP NOMO3 } \\
\text { FKBP1A PPIG PPIH HGS BAG6 DDAH2 CHMP2B SNAP47 SERPING1 PSMA2 ABHD17A } \\
\text { MIF4GD NDUFA10 VKORC1 LAS1L H2AFY2 RPS20 CHEK2 TERF1 DCTN2 DDX5 } \\
\text { EEF1A1 CAV1 UBE2I SGTA ATP6V1G1 }\end{array}$ \\
\hline GO:0099572 & postsynaptic specialization & $7 / 119$ & PHB STAT3 KPNA2 HNRNPA3 DNAJB1 CHMP2B ABHD17A \\
\hline GO:0018995 & host & $4 / 119$ & KPNB1 XPO1 CD209 CLEC4M \\
\hline GO:0044217 & other organism part & $4 / 119$ & KPNB1 XPO1 CD209 CLEC4M \\
\hline GO:0044216 & other organism cell & $4 / 119$ & KPNB1 XPO1 CD209 CLEC4M \\
\hline GO:0005615 & extracellular space & $41 / 119$ & $\begin{array}{l}\text { TWF2 KPNB1 PHB PPP1CA FGL2 TGFB1 HNRNPA1 ACE2 SFTPD PPIA CD9 TMPRSS2 } \\
\text { ANPEP DPP4 PTBP1 CEACAM1 ANXA2 HNRNPA2B1 DNAJB1 HSPD1 HSPA9 EIF3E } \\
\text { EIF3I CHMP4B SNX9 PABPC1 NCL MARK3 HGS BAG6 DDAH2 CHMP2B TPSAB1 } \\
\text { SERPING1 PSMA2 H2AFY2 RPS20 DCTN2 DDX5 EEF1A1 ATP6V1G1 }\end{array}$ \\
\hline GO:0031012 & extracellular matrix & $4 / 119$ & TGFB1 ANXA2 TPSAB1 SERPING1 \\
\hline GO:0072562 & blood microparticle & $2 / 119$ & TGFB1 SERPING1 \\
\hline GO:0031300 & $\begin{array}{l}\text { intrinsic component of } \\
\text { organelle membrane }\end{array}$ & $1 / 119$ & ABHD17A \\
\hline GO:0032420 & stereocilium & $1 / 119$ & TWF2 \\
\hline
\end{tabular}




\begin{tabular}{|c|c|c|c|}
\hline ID & Description & GeneRatio & geneID \\
\hline GO:0044446 & intracellular organelle part & $94 / 119$ & $\begin{array}{l}\text { SCFD1 PSMC2 TWF2 YKT6 KPNB1 STX5 STAT5A RSL24D1 ACBD5 PHB JUN STAT3 } \\
\text { PPP1CA SPECC1 FGL2 XPO1 TGFB1 SMAD3 ATF5 ND4L COX2 HNRNPA1 IRF3 KPNA2 } \\
\text { SFTPD PPIA BCL2L1 BCL2L2 MCL1 BCL2A1 BCL2 SKP2 KPNA4 PRKRA CD9 ANPEP } \\
\text { ZCRB1 DPP4 SYNCRIP PTBP1 CEACAM1 ANXA2 HNRNPA2B1 HNRNPA3 ACO2 } \\
\text { DNAJB1 HSPD1 HSPA9 COPB2 RPL13A EIF3E CHMP4B GBF1 KIF11 PSMD1 SRP54 } \\
\text { NUDCD1 SNX9 NONO GSK3B PABPC1 NPM1 RPL19 PARP1 NCL DDX1 RYBP NOMO3 } \\
\text { FKBP1A PPIG PPIH HGS BAG6 DDAH2 CHMP2B SNAP47 SERPING1 PSMA2 ABHD17A } \\
\text { MIF4GD NDUFA10 VKORC1 LAS1L H2AFY2 RPS20 CHEK2 TERF1 DCTN2 DDX5 } \\
\text { EEF1A1 CAV1 UBE2I SGTA ATP6V1G1 }\end{array}$ \\
\hline GO:0044449 & contractile fiber part & $1 / 119$ & FKBP1A \\
\hline GO:0098892 & $\begin{array}{l}\text { extrinsic component of } \\
\text { postsynaptic specialization } \\
\text { membrane }\end{array}$ & $1 / 119$ & KPNA2 \\
\hline GO:0098948 & $\begin{array}{l}\text { intrinsic component of } \\
\text { postsynaptic specialization } \\
\text { membrane }\end{array}$ & $1 / 119$ & ABHD17A \\
\hline GO:0099634 & $\begin{array}{l}\text { postsynaptic specialization } \\
\text { membrane }\end{array}$ & $2 / 119$ & KPNA2 ABHD17A \\
\hline GO:0019898 & $\begin{array}{l}\text { extrinsic component of } \\
\text { membrane }\end{array}$ & $7 / 119$ & PHB KPNA2 ANXA2 NMT1 SNX9 FKBP1A SGTA \\
\hline GO:0005905 & clathrin-coated pit & $1 / 119$ & HSPD1 \\
\hline GO:0031224 & $\begin{array}{l}\text { intrinsic component of } \\
\text { membrane }\end{array}$ & $26 / 119$ & $\begin{array}{l}\text { YKT6 STX5 ACBD5 PHB ND4L COX2 ACE2 CLEC4G CD209 CLEC4M BCL2L1 MCL1 } \\
\text { BCL2 CD9 TMPRSS2 IKBKB ANPEP DPP4 CEACAM1 HSPD1 NOMO3 FKBP1A CAMLG } \\
\text { ABHD17A VKORC1 CAV1 }\end{array}$ \\
\hline GO:0044455 & $\begin{array}{l}\text { mitochondrial membrane } \\
\text { part }\end{array}$ & $5 / 119$ & PHB ND4L COX2 HSPD1 NDUFA10 \\
\hline GO:0044459 & plasma membrane part & $19 / 119$ & $\begin{array}{l}\text { YKT6 PHB ACE2 CD209 CLEC4M KPNA2 CD9 TMPRSS2 IKBKB ANPEP DPP4 } \\
\text { CEACAM1 ANXA2 CHMP4B SNX9 FKBP1A ABHD17A EEF1A1 CAV1 }\end{array}$ \\
\hline GO:0070469 & respiratory chain & $3 / 119$ & ND4L COX2 NDUFA10 \\
\hline GO:0098552 & side of membrane & $8 / 119$ & CD209 CD9 IKBKB ANPEP CHMP4B SNX9 FKBP1A EEF1A1 \\
\hline GO:0008021 & synaptic vesicle & $3 / 119$ & BCL2L1 SNAP47 SGTA \\
\hline GO:0030672 & synaptic vesicle membrane & $3 / 119$ & BCL2L1 SNAP47 SGTA \\
\hline GO:0048786 & presynaptic active zone & $1 / 119$ & PHB \\
\hline GO:0097060 & synaptic membrane & $3 / 119$ & PHB KPNA2 ABHD17A \\
\hline GO:0098793 & presynapse & $5 / 119$ & PHB PPP1CA BCL2L1 SNAP47 SGTA \\
\hline GO:0098794 & postsynapse & $11 / 119$ & $\begin{array}{l}\text { PSMC2 PHB STAT3 PPP1CA KPNA2 HNRNPA3 DNAJB1 GSK3A GSK3B CHMP2B } \\
\text { ABHD17A }\end{array}$ \\
\hline GO:0098845 & postsynaptic endosome & $1 / 119$ & ABHD17A \\
\hline GO:0098850 & $\begin{array}{l}\text { extrinsic component of } \\
\text { synaptic vesicle membrane }\end{array}$ & $1 / 119$ & SGTA \\
\hline GO:0098895 & $\begin{array}{l}\text { postsynaptic endosome } \\
\text { membrane }\end{array}$ & $1 / 119$ & ABHD17A \\
\hline GO:0098949 & $\begin{array}{l}\text { intrinsic component of } \\
\text { postsynaptic endosome } \\
\text { membrane }\end{array}$ & $1 / 119$ & ABHD17A \\
\hline
\end{tabular}




\begin{tabular}{|c|c|c|c|}
\hline ID & Description & GeneRatio & geneID \\
\hline GO:0099240 & $\begin{array}{l}\text { intrinsic component of } \\
\text { synaptic membrane }\end{array}$ & $1 / 119$ & ABHD17A \\
\hline GO:0099243 & $\begin{array}{l}\text { extrinsic component of } \\
\text { synaptic membrane }\end{array}$ & $2 / 119$ & PHB KPNA2 \\
\hline GO:0005622 & intracellular & $114 / 119$ & $\begin{array}{l}\text { BTF3 SCFD1 PSMC2 TFEB TWF2 YKT6 KPNB1 STX5 STAT5A RSL24D1 ACBD5 PHB } \\
\text { JUN STAT3 PPP1CA SPECC1 FGL2 XPO1 TGFB1 SMAD3 ATF5 ND4L COX2 HNRNPA1 } \\
\text { ACE2 CD209 CLEC4M IRF3 KPNA2 SFTPD PPIA BCL2L1 BCL2L2 MCL1 BCL2A1 BCL2 } \\
\text { SKP2 KPNA4 PRKRA CD9 IKBKB ANPEP ZCRB1 DPP4 SYNCRIP PTBP1 CEACAM1 } \\
\text { ANXA2 HNRNPA2B1 HNRNPA3 ACO2 DNAJB1 HSPD1 HSPA9 COPB2 RPL13A EIF3E } \\
\text { EIF3I NMT1 CHMP4B EIF3F GBF1 RRM2 KIF11 PSMD1 SRP54 NUDCD1 NACA SNX9 } \\
\text { NONO GSK3A GSK3B PABPC1 PABPC4 NPM1 G3BP1 G3BP2 RPL19 PARP1 NCL DDX1 } \\
\text { RYBP NOMO3 FKBP1A PPIG MARK3 PPIH RCAN3 HGS BAG6 DDAH2 CAMLG } \\
\text { CHMP2B SNAP47 MKRN2 SERPING1 PSMA2 ABHD17A PFDN5 MIF4GD NDUFA10 } \\
\text { VKORC1 LAS1L H2AFY2 RPS20 CHEK2 TERF1 DCTN2 DDX5 EEF1A1 CAV1 UBE2I } \\
\text { SGTA ATP6V1G1 }\end{array}$ \\
\hline GO:0005642 & annulate lamellae & $1 / 119$ & XPO1 \\
\hline GO:0008287 & $\begin{array}{l}\text { protein serine/threonine } \\
\text { phosphatase complex }\end{array}$ & $1 / 119$ & PPP1CA \\
\hline GO:0009986 & cell surface & $10 / 119$ & PHB TGFB1 ACE2 CD209 CD9 ANPEP DPP4 CEACAM1 ANXA2 HSPD1 \\
\hline GO:0012505 & endomembrane system & $42 / 119$ & $\begin{array}{l}\text { SCFD1 PSMC2 YKT6 KPNB1 STX5 PHB FGL2 XPO1 TGFB1 SMAD3 SFTPD PPIA } \\
\text { BCL2L1 BCL2 KPNA4 CD9 ANPEP SYNCRIP CEACAM1 ANXA2 HSPD1 COPB2 } \\
\text { CHMP4B GBF1 PSMD1 SNX9 PARP1 NOMO3 FKBP1A HGS CAMLG CHMP2B SNAP47 } \\
\text { SERPING1 PSMA2 ABHD17A VKORC1 CHEK2 EEF1A1 CAV1 UBE2I SGTA }\end{array}$ \\
\hline GO:0030427 & site of polarized growth & $2 / 119$ & TWF2 DCTN2 \\
\hline GO:0030496 & midbody & $2 / 119$ & ANXA2 CHMP4B \\
\hline GO:0031252 & cell leading edge & $6 / 119$ & TWF2 DPP4 ANXA2 GBF1 SNX9 EEF1A1 \\
\hline GO:0031975 & envelope & $18 / 119$ & $\begin{array}{l}\text { KPNB1 PHB STAT3 XPO1 SMAD3 ND4L COX2 BCL2L1 BCL2L2 MCL1 BCL2A1 BCL2 } \\
\text { KPNA4 HSPD1 CHMP4B PARP1 NDUFA10 UBE2I }\end{array}$ \\
\hline GO:0035749 & $\begin{array}{l}\text { myelin sheath adaxonal } \\
\text { region }\end{array}$ & $1 / 119$ & ANXA2 \\
\hline GO:0042995 & cell projection & $22 / 119$ & $\begin{array}{l}\text { PSMC2 TWF2 YKT6 PPP1CA TGFB1 ACE2 DPP4 CEACAM1 ANXA2 HNRNPA3 } \\
\text { DNAJB1 SNX9 GSK3A GSK3B MARK3 SNAP47 ABHD17A DCTN2 C11 orf74 EEF1A1 } \\
\text { CAV1 UBE2I }\end{array}$ \\
\hline GO:0043209 & myelin sheath & $6 / 119$ & PHB BCL2 ANXA2 ACO2 HSPD1 NDUFA10 \\
\hline GO:0043218 & compact myelin & $1 / 119$ & ANXA2 \\
\hline GO:0043220 & Schmidt-Lanterman incisure & $1 / 119$ & ANXA2 \\
\hline GO:0044297 & cell body & $7 / 119$ & YKT6 PPP1CA TGFB1 DNAJB1 GSK3A G3BP1 SNAP47 \\
\hline GO:0044424 & intracellular part & $113 / 119$ & $\begin{array}{l}\text { BTF3 SCFD1 PSMC2 TFEB TWF2 YKT6 KPNB1 STX5 STAT5A RSL24D1 ACBD5 PHB } \\
\text { JUN STAT3 PPP1CA SPECC1 FGL2 XPO1 TGFB1 SMAD3 ATF5 ND4L COX2 HNRNPA1 } \\
\text { ACE2 CD209 CLEC4M IRF3 KPNA2 SFTPD PPIA BCL2L1 BCL2L2 MCL1 BCL2A1 BCL2 } \\
\text { SKP2 KPNA4 PRKRA CD9 IKBKB ANPEP ZCRB1 DPP4 SYNCRIP PTBP1 CEACAM1 } \\
\text { ANXA2 HNRNPA2B1 HNRNPA3 ACO2 DNAJB1 HSPD1 HSPA9 COPB2 RPL13A EIF3E } \\
\text { EIF3I NMT1 CHMP4B EIF3F GBF1 RRM2 KIF11 PSMD1 SRP54 NUDCD1 NACA SNX9 } \\
\text { NONO GSK3A GSK3B PABPC1 PABPC4 NPM1 G3BP1 G3BP2 RPL19 PARP1 NCL DDX1 } \\
\text { RYBP NOMO3 FKBP1A PPIG MARK3 PPIH RCAN3 HGS BAG6 DDAH2 CAMLG } \\
\text { CHMP2B SNAP47 SERPING1 PSMA2 ABHD17A PFDN5 MIF4GD NDUFA10 VKORC1 } \\
\text { LAS1L H2AFY2 RPS20 CHEK2 TERF1 DCTN2 DDX5 EEF1A1 CAV1 UBE2I SGTA } \\
\text { ATP6V1G1 }\end{array}$ \\
\hline GO:0044463 & cell projection part & $16 / 119$ & $\begin{array}{c}\text { PSMC2 TWF2 YKT6 PPP1CA ACE2 DPP4 CEACAM1 DNAJB1 GSK3A GSK3B MARK3 } \\
\text { SNAP47 ABHD17A DCTN2 EEF1A1 UBE2I }\end{array}$ \\
\hline
\end{tabular}




\begin{tabular}{|c|c|c|c|}
\hline ID & Description & GeneRatio & geneID \\
\hline GO:0045177 & apical part of cell & $3 / 119$ & CD9 DPP4 CEACAM1 \\
\hline GO:0045178 & basal part of cell & $1 / 119$ & CEACAM1 \\
\hline GO:0071944 & cell periphery & $33 / 119$ & $\begin{array}{c}\text { SCFD1 YKT6 PHB STAT3 PPP1CA TGFB1 SMAD3 ACE2 CLEC4G CD209 CLEC4M } \\
\text { KPNA2 CD9 TMPRSS2 IKBKB ANPEP DPP4 CEACAM1 ANXA2 HSPD1 NMT1 CHMP4B } \\
\text { SNX9 GSK3B NCL FKBP1A MARK3 CHMP2B SNAP47 ABHD17A EEF1A1 CAV1 } \\
\text { ATP6V1G1 }\end{array}$ \\
\hline GO:0097223 & sperm part & $2 / 119$ & DNAJB1 CAV1 \\
\hline GO:0097458 & neuron part & $20 / 119$ & $\begin{array}{l}\text { PSMC2 TWF2 YKT6 PHB STAT3 PPP1CA TGFB1 BCL2L1 HNRNPA3 DNAJB1 GSK3A } \\
\text { GSK3B G3BP1 MARK3 CHMP2B SNAP47 ABHD17A DCTN2 UBE2I SGTA }\end{array}$ \\
\hline GO:0098862 & $\begin{array}{l}\text { cluster of actin-based cell } \\
\text { projections }\end{array}$ & $2 / 119$ & TWF2 ACE2 \\
\hline GO:1990204 & oxidoreductase complex & $2 / 119$ & ND4L NDUFA10 \\
\hline GO:0044456 & synapse part & $14 / 119$ & $\begin{array}{c}\text { PSMC2 PHB STAT3 PPP1CA KPNA2 BCL2L1 HNRNPA3 DNAJB1 GSK3A GSK3B } \\
\text { CHMP2B SNAP47 ABHD17A SGTA }\end{array}$ \\
\hline GO:0098685 & $\begin{array}{l}\text { Schaffer collateral - CA1 } \\
\text { synapse }\end{array}$ & $1 / 119$ & STAT3 \\
\hline GO:0098978 & glutamatergic synapse & $9 / 119$ & PHB STAT3 PPP1CA KPNA2 DNAJB1 GSK3B CHMP2B SNAP47 ABHD17A \\
\hline GO:0098982 & GABA-ergic synapse & $1 / 119$ & PHB \\
\hline GO:0098984 & neuron to neuron synapse & $6 / 119$ & PHB STAT3 HNRNPA3 DNAJB1 CHMP2B ABHD17A \\
\hline GO:0099081 & supramolecular polymer & $5 / 119$ & TWF2 SPECC1 KIF11 FKBP1A DCTN2 \\
\hline
\end{tabular}

\subsubsection{BIOLOGICAL PROCESS}

\begin{tabular}{|c|c|c|c|}
\hline ID & Description & GeneRatio & geneID \\
\hline GO:0019953 & sexual reproduction & $7 / 119$ & STAT3 TGFB1 BCL2L1 BCL2L2 BCL2 CD9 BAG6 \\
\hline GO:0022414 & reproductive process & $10 / 119$ & TFEB PHB STAT3 TGFB1 BCL2L1 BCL2L2 BCL2 CD9 BAG6 TERF1 \\
\hline GO:0032504 & $\begin{array}{l}\text { multicellular organism } \\
\text { reproduction }\end{array}$ & $6 / 119$ & TGFB1 BCL2L1 BCL2L2 BCL2 CD9 BAG6 \\
\hline GO:0055114 & oxidation-reduction process & $9 / 119$ & PPP1CA ND4L COX2 ACO2 RRM2 GSK3A GSK3B NDUFA10 VKORC1 \\
\hline GO:0006807 & $\begin{array}{l}\text { nitrogen compound metabolic } \\
\text { process }\end{array}$ & $90 / 119$ & $\begin{array}{l}\text { BTF3 PSMC2 TFEB KPNB1 STX5 STAT5A RSL24D1 PHB JUN STAT3 PPP1CA FGL2 } \\
\text { XPO1 TGFB1 SMAD3 ATF5 ND4L COX2 HNRNPA1 ACE2 IRF3 KPNA2 SFTPD PPIA } \\
\text { BCL2 SKP2 PRKRA TMPRSS2 IKBKB ANPEP ZCRB1 DPP4 SYNCRIP PTBP1 } \\
\text { CEACAM1 ANXA2 HNRNPA2B1 HNRNPA3 DNAJB1 HSPD1 RPL13A EIF3E EIF3I } \\
\text { NMT1 CHMP4B EIF3F RRM2 PSMD1 SNX9 NONO GSK3A GSK3B PABPC1 PABPC4 } \\
\text { NPM1 G3BP1 G3BP2 RPL19 PARP1 NCL DDX1 RYBP FKBP1A PPIG MARK3 PPIH } \\
\text { RCAN3 HGS BAG6 DDAH2 MKRN2 TPSAB1 SERPING1 MKRN3 PSMA2 ABHD17A } \\
\text { PFDN5 MIF4GD NDUFA10 VKORC1 LAS1L H2AFY2 RPS20 CHEK2 TERF1 DDX5 } \\
\text { EEF1A1 CAV1 UBE2I SGTA }\end{array}$ \\
\hline GO:0009056 & catabolic process & $45 / 119$ & $\begin{array}{l}\text { SCFD1 PSMC2 TFEB KPNB1 STX5 ACBD5 PHB STAT3 PPP1CA XPO1 TGFB1 } \\
\text { SMAD3 MCL1 BCL2 SKP2 ANPEP SYNCRIP CEACAM1 ANXA2 RPL13A EIF3E } \\
\text { CHMP4B PSMD1 SNX9 GSK3A GSK3B PABPC1 PABPC4 NPM1 RPL19 RYBP HGS }\end{array}$ \\
\hline
\end{tabular}




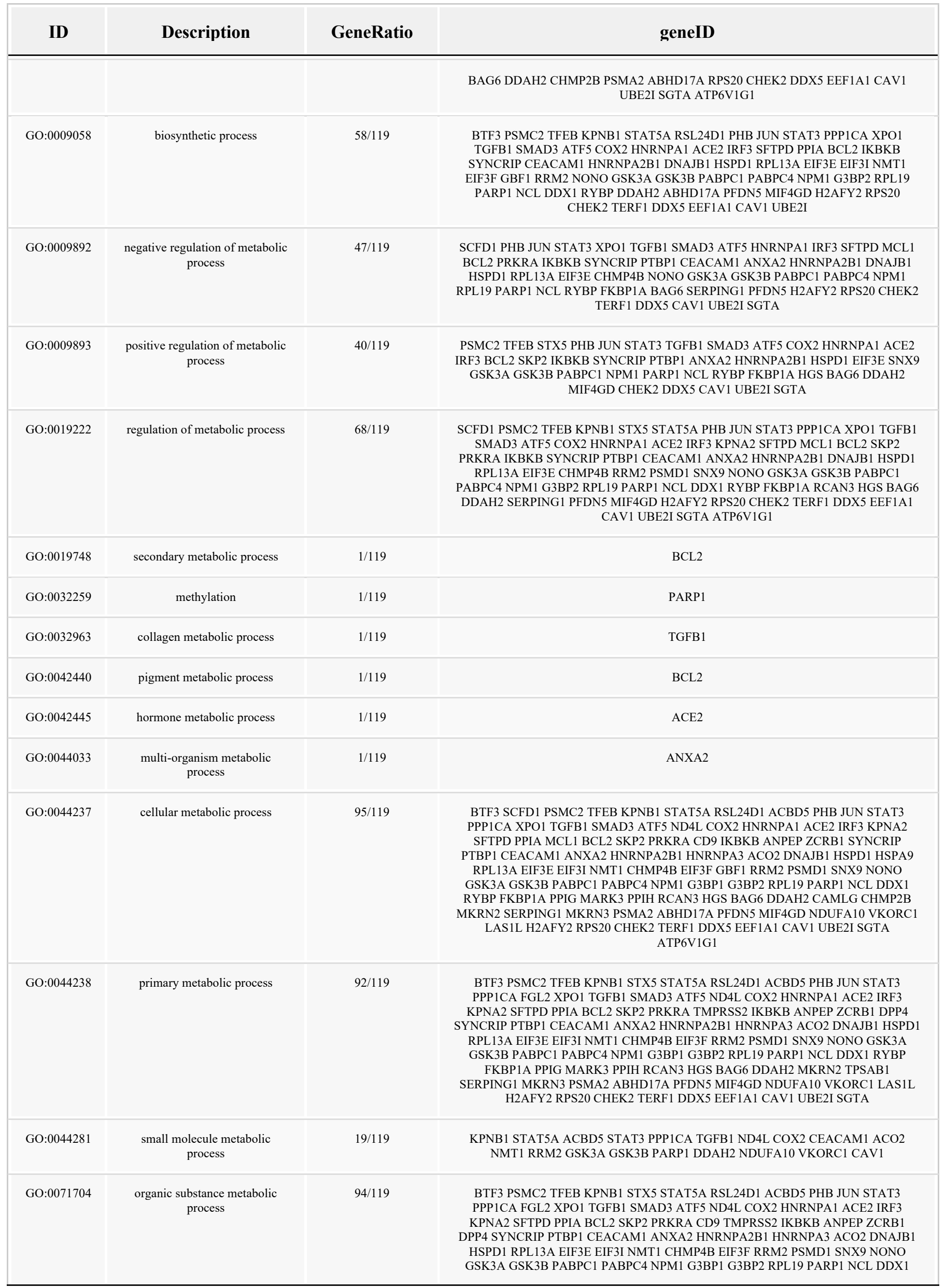




\begin{tabular}{|c|c|c|c|}
\hline ID & Description & GeneRatio & geneID \\
\hline & & & $\begin{array}{l}\text { RYBP FKBP1A PPIG MARK3 PPIH RCAN3 HGS BAG6 DDAH2 CAMLG MKRN2 } \\
\text { TPSAB1 SERPING1 MKRN3 PSMA2 ABHD17A PFDN5 MIF4GD NDUFA10 VKORC1 } \\
\text { LAS1L H2AFY2 RPS20 CHEK2 TERF1 DDX5 EEF1A1 CAV1 UBE2I SGTA }\end{array}$ \\
\hline GO:1990845 & adaptive thermogenesis & $1 / 119$ & CAV1 \\
\hline GO:0001909 & leukocyte mediated cytotoxicity & $1 / 119$ & CEACAM1 \\
\hline GO:0031341 & regulation of cell killing & $1 / 119$ & CEACAM1 \\
\hline GO:0031342 & negative regulation of cell killing & $1 / 119$ & CEACAM1 \\
\hline GO:0001776 & leukocyte homeostasis & $2 / 119$ & TGFB1 BCL2 \\
\hline GO:0002200 & $\begin{array}{l}\text { somatic diversification of } \\
\text { immune receptors }\end{array}$ & $2 / 119$ & TGFB1 HSPD1 \\
\hline GO:0002252 & immune effector process & $21 / 119$ & $\begin{array}{l}\text { PSMC2 KPNB1 PHB STAT3 FGL2 TGFB1 CLEC4G IRF3 PPIA BCL2 SKP2 ANPEP } \\
\text { CEACAM1 ANXA2 HSPD1 GBF1 PSMD1 DDX1 SERPING1 PSMA2 EEF1A1 }\end{array}$ \\
\hline GO:0002253 & activation of immune response & $12 / 119$ & $\begin{array}{l}\text { PHB CD209 IRF3 SFTPD BCL2 IKBKB CEACAM1 HSPD1 NONO BAG6 SERPING1 } \\
\text { CAV1 }\end{array}$ \\
\hline GO:0002262 & myeloid cell homeostasis & $2 / 119$ & STAT3 HSPA9 \\
\hline GO:0002440 & $\begin{array}{l}\text { production of molecular } \\
\text { mediator of immune response }\end{array}$ & $3 / 119$ & TGFB1 HSPD1 DDX1 \\
\hline GO:0002507 & tolerance induction & $1 / 119$ & TGFB1 \\
\hline GO:0002520 & immune system development & $11 / 119$ & $\begin{array}{l}\text { JUN STAT3 TGFB1 SMAD3 BCL2 CEACAM1 ANXA2 HSPD1 HSPA9 PABPC4 } \\
\text { PARP1 }\end{array}$ \\
\hline GO:0002682 & $\begin{array}{l}\text { regulation of immune system } \\
\text { process }\end{array}$ & $21 / 119$ & $\begin{array}{l}\text { PHB JUN STAT3 TGFB1 SMAD3 CLEC4G CD209 IRF3 SFTPD BCL2 IKBKB DPP4 } \\
\text { CEACAM1 HSPD1 HSPA9 NONO DDX1 FKBP1A BAG6 SERPING1 CAV1 }\end{array}$ \\
\hline GO:0002683 & $\begin{array}{l}\text { negative regulation of immune } \\
\text { system process }\end{array}$ & $6 / 119$ & TGFB1 CLEC4G SFTPD CEACAM1 HSPA9 SERPING1 \\
\hline GO:0002684 & $\begin{array}{l}\text { positive regulation of immune } \\
\text { system process }\end{array}$ & $17 / 119$ & $\begin{array}{l}\text { PHB JUN STAT3 TGFB1 CD209 IRF3 SFTPD BCL2 IKBKB DPP4 CEACAM1 HSPD1 } \\
\text { NONO DDX1 BAG6 SERPING1 CAV1 }\end{array}$ \\
\hline GO:0006955 & immune response & $35 / 119$ & $\begin{array}{l}\text { PSMC2 TFEB KPNB1 PHB JUN STAT3 FGL2 TGFB1 SMAD3 CLEC4G CD209 } \\
\text { CLEC4M IRF3 SFTPD PPIA BCL2 SKP2 PRKRA IKBKB ANPEP SYNCRIP CEACAM1 } \\
\text { ANXA2 HSPD1 RPL13A GBF1 PSMD1 NONO DDX1 FKBP1A BAG6 SERPING1 } \\
\text { PSMA2 EEF1A1 CAV1 }\end{array}$ \\
\hline GO:0019882 & $\begin{array}{l}\text { antigen processing and } \\
\text { presentation }\end{array}$ & $5 / 119$ & CD209 CLEC4M IKBKB KIF11 DCTN2 \\
\hline GO:0031294 & lymphocyte costimulation & $2 / 119$ & DPP4 CAV1 \\
\hline GO:0045058 & $\mathrm{T}$ cell selection & $2 / 119$ & STAT3 BCL2 \\
\hline GO:0045321 & leukocyte activation & $23 / 119$ & $\begin{array}{l}\text { PSMC2 KPNB1 JUN STAT3 FGL2 TGFB1 SMAD3 CLEC4G CD209 SFTPD PPIA BCL2 } \\
\text { ANPEP DPP4 CEACAM1 ANXA2 HSPD1 PSMD1 FKBP1A BAG6 PSMA2 EEF1A1 } \\
\text { CAV1 }\end{array}$ \\
\hline GO:0050900 & leukocyte migration & $6 / 119$ & TGFB1 SFTPD PPIA CEACAM1 GBF1 CAV1 \\
\hline GO:0016049 & cell growth & $8 / 119$ & TWF2 PHB TGFB1 SMAD3 BCL2 CEACAM1 GSK3A GSK3B \\
\hline GO:0040008 & regulation of growth & $11 / 119$ & $\begin{array}{l}\text { TWF2 STAT5A PHB STAT3 TGFB1 SMAD3 BCL2L1 BCL2 CEACAM1 GSK3A } \\
\text { GSK3B }\end{array}$ \\
\hline
\end{tabular}




\begin{tabular}{|c|c|c|c|}
\hline ID & Description & GeneRatio & geneID \\
\hline GO:0045926 & negative regulation of growth & $5 / 119$ & PHB TGFB1 SMAD3 BCL2 GSK3A \\
\hline GO:0045927 & positive regulation of growth & $2 / 119$ & TWF2 BCL2 \\
\hline GO:0048589 & developmental growth & $10 / 119$ & TWF2 STAT5A STAT3 TGFB1 SMAD3 ATF5 BCL2 ANXA2 GSK3A GSK3B \\
\hline GO:0002209 & behavioral defense response & $2 / 119$ & BCL2 DPP4 \\
\hline GO:0007611 & learning or memory & $1 / 119$ & JUN \\
\hline GO:0007626 & locomotory behavior & $1 / 119$ & DPP4 \\
\hline GO:0007631 & feeding behavior & $2 / 119$ & STAT3 ACE2 \\
\hline GO:0035640 & exploration behavior & $1 / 119$ & DPP4 \\
\hline GO:0050795 & regulation of behavior & $1 / 119$ & STAT3 \\
\hline GO:0061744 & motor behavior & $1 / 119$ & DPP4 \\
\hline GO:0008284 & $\begin{array}{l}\text { positive regulation of cell } \\
\text { proliferation }\end{array}$ & $11 / 119$ & STAT5A JUN STAT3 TGFB1 CD209 BCL2L1 BCL2 SKP2 DPP4 ANXA2 NPM1 \\
\hline GO:0008285 & $\begin{array}{l}\text { negative regulation of cell } \\
\text { proliferation }\end{array}$ & $15 / 119$ & $\begin{array}{l}\text { PHB JUN STAT3 TGFB1 SMAD3 ATF5 CLEC4G SFTPD BCL2 PRKRA CD9 } \\
\text { CEACAM1 NPM1 HGS CAV1 }\end{array}$ \\
\hline GO:0033002 & muscle cell proliferation & $3 / 119$ & JUN STAT3 SKP2 \\
\hline GO:0033687 & osteoblast proliferation & $2 / 119$ & SMAD3 BCL2 \\
\hline GO:0035726 & $\begin{array}{l}\text { common myeloid progenitor cell } \\
\text { proliferation }\end{array}$ & $1 / 119$ & CEACAM1 \\
\hline GO:0042127 & regulation of cell proliferation & $23 / 119$ & $\begin{array}{l}\text { STAT5A PHB JUN STAT3 TGFB1 SMAD3 ATF5 ACE2 CLEC4G CD209 SFTPD } \\
\text { BCL2L1 BCL2 SKP2 PRKRA CD9 DPP4 CEACAM1 ANXA2 NPM1 HGS BAG6 CAV1 }\end{array}$ \\
\hline GO:0048144 & fibroblast proliferation & $3 / 119$ & JUN TGFB1 ANXA2 \\
\hline GO:0050673 & epithelial cell proliferation & $8 / 119$ & STAT5A JUN STAT3 TGFB1 SMAD3 BCL2L2 CEACAM1 CAV1 \\
\hline GO:0061351 & $\begin{array}{l}\text { neural precursor cell } \\
\text { proliferation }\end{array}$ & $2 / 119$ & TGFB1 ATF5 \\
\hline GO:0070661 & leukocyte proliferation & $6 / 119$ & TGFB1 CLEC4G CD209 SFTPD BCL2 HSPD1 \\
\hline GO:0072089 & stem cell proliferation & $1 / 119$ & TGFB1 \\
\hline GO:0007059 & chromosome segregation & $6 / 119$ & KPNB1 CHMP4B BAG6 CHMP2B TERF1 UBE2I \\
\hline GO:0007017 & microtubule-based process & $11 / 119$ & KPNB1 XPO1 ATF5 CHMP4B KIF11 GSK3B NPM1 MARK3 CHMP2B CHEK2 DCTN2 \\
\hline GO:0000075 & cell cycle checkpoint & $3 / 119$ & TGFB1 BCL2L1 CHEK2 \\
\hline GO:0000920 & $\begin{array}{l}\text { septum digestion after } \\
\text { cytokinesis }\end{array}$ & $1 / 119$ & CHMP2B \\
\hline GO:0001775 & cell activation & $25 / 119$ & $\begin{array}{l}\text { PSMC2 KPNB1 JUN STAT3 FGL2 TGFB1 SMAD3 CLEC4G CD209 SFTPD PPIA BCL2 } \\
\text { CD9 ANPEP DPP4 CEACAM1 ANXA2 HSPD1 GBF1 PSMD1 FKBP1A BAG6 PSMA2 } \\
\text { EEF1A1 CAV1 }\end{array}$ \\
\hline GO:0006457 & protein folding & $9 / 119$ & PPIA DNAJB1 HSPD1 HSPA9 FKBP1A PPIG PPIH PFDN5 SGTA \\
\hline
\end{tabular}




\begin{tabular}{|c|c|c|c|}
\hline ID & Description & GeneRatio & geneID \\
\hline GO:0007165 & signal transduction & $53 / 119$ & $\begin{array}{l}\text { KPNB1 STAT5A PHB JUN STAT3 PPP1CA TGFB1 SMAD3 HNRNPA1 CD209 } \\
\text { CLEC4M IRF3 KPNA2 SFTPD PPIA BCL2L1 BCL2L2 MCL1 BCL2A1 BCL2 SKP2 } \\
\text { PRKRA CD9 IKBKB PTBP1 CEACAM1 ANXA2 HNRNPA2B1 HSPD1 HSPA9 NMT1 } \\
\text { GBF1 NONO GSK3A GSK3B NPM1 G3BP1 G3BP2 PARP1 DDX1 FKBP1A MARK3 } \\
\text { RCAN3 HGS BAG6 DDAH2 CAMLG PFDN5 CHEK2 DDX5 CAV1 UBE2I ATP6V1G1 }\end{array}$ \\
\hline GO:0006903 & vesicle targeting & $4 / 119$ & SCFD1 YKT6 STX5 GBF1 \\
\hline GO:0006928 & $\begin{array}{l}\text { movement of cell or subcellular } \\
\text { component }\end{array}$ & $15 / 119$ & $\begin{array}{l}\text { KPNB1 STAT5A JUN STAT3 TGFB1 SMAD3 SFTPD PPIA BCL2 CD9 DPP4 } \\
\text { CEACAM1 GBF1 KIF11 CAV1 }\end{array}$ \\
\hline GO:0007049 & cell cycle & $24 / 119$ & $\begin{array}{l}\text { KPNB1 JUN STAT3 PPP1CA XPO1 TGFB1 SMAD3 ATF5 BCL2L1 BCL2 SKP2 } \\
\text { CHMP4B GBF1 RRM2 KIF11 SNX9 NPM1 RYBP BAG6 CHMP2B CHEK2 TERF1 } \\
\text { DCTN2 UBE2I }\end{array}$ \\
\hline GO:0007154 & cell communication & $55 / 119$ & $\begin{array}{l}\text { KPNB1 STAT5A PHB JUN STAT3 PPP1CA TGFB1 SMAD3 HNRNPA1 CD209 } \\
\text { CLEC4M IRF3 KPNA2 SFTPD PPIA BCL2L1 BCL2L2 MCL1 BCL2A1 BCL2 SKP2 } \\
\text { PRKRA CD9 IKBKB DPP4 PTBP1 CEACAM1 ANXA2 HNRNPA2B1 HSPD1 HSPA9 } \\
\text { NMT1 GBF1 NONO GSK3A GSK3B NPM1 G3BP1 G3BP2 PARP1 DDX1 FKBP1A } \\
\text { MARK3 RCAN3 HGS BAG6 DDAH2 CAMLG SNAP47 PFDN5 CHEK2 DDX5 CAV1 } \\
\text { UBE2I ATP6V1G1 }\end{array}$ \\
\hline GO:0007163 & $\begin{array}{l}\text { establishment or maintenance of } \\
\text { cell polarity }\end{array}$ & $3 / 119$ & GBF1 GSK3B MARK3 \\
\hline GO:0007272 & ensheathment of neurons & $2 / 119$ & TGFB1 CD9 \\
\hline GO:0008219 & cell death & $33 / 119$ & $\begin{array}{l}\text { KPNB1 PHB JUN STAT3 PPP1CA TGFB1 SMAD3 ATF5 COX2 IRF3 BCL2L1 BCL2L2 } \\
\text { MCL1 BCL2A1 BCL2 SKP2 PRKRA IKBKB HSPD1 HSPA9 NMT1 CHMP4B NONO } \\
\text { GSK3A GSK3B NPM1 PARP1 RYBP BAG6 DDAH2 CHEK2 DDX5 CAV1 }\end{array}$ \\
\hline GO:0008037 & cell recognition & $2 / 119$ & CD209 CLEC4M \\
\hline GO:0016043 & cellular component organization & $66 / 119$ & $\begin{array}{l}\text { SCFD1 PSMC2 TFEB TWF2 YKT6 KPNB1 STX5 RSL24D1 PHB JUN STAT3 PPP1CA } \\
\text { SPECC1 XPO1 TGFB1 SMAD3 ATF5 HNRNPA1 ACE2 SFTPD PPIA BCL2L1 BCL2 } \\
\text { CD9 IKBKB DPP4 CEACAM1 ANXA2 HNRNPA2B1 DNAJB1 HSPD1 HSPA9 RPL13A } \\
\text { EIF3E EIF3I NMT1 CHMP4B EIF3F GBF1 KIF11 SRP54 SNX9 GSK3A GSK3B NPM1 } \\
\text { G3BP1 G3BP2 PARP1 DDX1 RYBP FKBP1A MARK3 PPIH HGS BAG6 CHMP2B } \\
\text { SNAP47 TPSAB1 ABHD17A NDUFA10 H2AFY2 CHEK2 TERF1 DCTN2 CAV1 SGTA }\end{array}$ \\
\hline GO:0016458 & gene silencing & $7 / 119$ & STAT3 TGFB1 SMAD3 PRKRA HNRNPA2B1 PABPC1 DDX5 \\
\hline GO:0019725 & cellular homeostasis & $10 / 119$ & TGFB1 SMAD3 MCL1 BCL2 ANXA2 FKBP1A CHMP2B TERF1 CAV1 ATP6V1G1 \\
\hline GO:0022402 & cell cycle process & $19 / 119$ & $\begin{array}{l}\text { KPNB1 XPO1 TGFB1 SMAD3 ATF5 BCL2L1 BCL2 SKP2 CHMP4B RRM2 KIF11 } \\
\text { SNX9 NPM1 RYBP BAG6 CHMP2B CHEK2 TERF1 DCTN2 }\end{array}$ \\
\hline GO:0022406 & membrane docking & $4 / 119$ & SCFD1 YKT6 STX5 DCTN2 \\
\hline GO:0022412 & $\begin{array}{l}\text { cellular process involved in } \\
\text { reproduction in multicellular } \\
\text { organism }\end{array}$ & $4 / 119$ & TGFB1 BCL2L1 BCL2 CD9 \\
\hline GO:0030029 & actin filament-based process & $7 / 119$ & TWF2 SPECC1 TGFB1 SMAD3 BCL2 IKBKB CAV1 \\
\hline GO:0032940 & secretion by cell & $21 / 119$ & $\begin{array}{l}\text { SCFD1 PSMC2 YKT6 KPNB1 FGL2 TGFB1 IRF3 PPIA CD9 ANPEP DPP4 CEACAM1 } \\
\text { ANXA2 HSPD1 PSMD1 GSK3B HGS SNAP47 SERPING1 PSMA2 EEF1A1 }\end{array}$ \\
\hline GO:0033059 & cellular pigmentation & $2 / 119$ & BCL2 DCTN2 \\
\hline GO:0044764 & multi-organism cellular process & $2 / 119$ & IRF3 PPIA \\
\hline GO:0048522 & $\begin{array}{l}\text { positive regulation of cellular } \\
\text { process }\end{array}$ & $54 / 119$ & $\begin{array}{l}\text { PSMC2 TFEB TWF2 STAT5A PHB JUN STAT3 PPP1CA TGFB1 SMAD3 ATF5 COX2 } \\
\text { HNRNPA1 ACE2 CD209 IRF3 SFTPD PPIA BCL2L1 MCL1 BCL2 SKP2 PRKRA } \\
\text { IKBKB DPP4 PTBP1 CEACAM1 ANXA2 HNRNPA2B1 HSPD1 EIF3E NMT1 SNX9 } \\
\text { GSK3A GSK3B PABPC1 NPM1 PARP1 NCL DDX1 RYBP FKBP1A HGS BAG6 } \\
\text { DDAH2 SNAP47 MIF4GD H2AFY2 CHEK2 TERF1 DDX5 CAV1 UBE2I SGTA }\end{array}$ \\
\hline
\end{tabular}




\begin{tabular}{|c|c|c|c|}
\hline ID & Description & GeneRatio & geneID \\
\hline GO:0048523 & $\begin{array}{l}\text { negative regulation of cellular } \\
\text { process }\end{array}$ & $57 / 119$ & $\begin{array}{c}\text { SCFD1 TWF2 PHB JUN STAT3 XPO1 TGFB1 SMAD3 ATF5 HNRNPA1 CLEC4G IRF3 } \\
\text { SFTPD BCL2L1 BCL2L2 MCL1 BCL2A1 BCL2 PRKRA CD9 IKBKB DPP4 SYNCRIP } \\
\text { PTBP1 CEACAM1 ANXA2 HNRNPA2B1 DNAJB1 HSPD1 HSPA9 RPL13A EIF3E } \\
\text { CHMP4B RRM2 NONO GSK3A GSK3B PABPC1 NPM1 G3BP1 PARP1 NCL RYBP } \\
\text { FKBP1A MARK3 HGS BAG6 DDAH2 SERPING1 PFDN5 H2AFY2 CHEK2 TERF1 } \\
\text { DDX5 CAV1 UBE2I SGTA }\end{array}$ \\
\hline GO:0048869 & cellular developmental process & $30 / 119$ & $\begin{array}{l}\text { SCFD1 PSMC2 TWF2 PHB JUN STAT3 TGFB1 SMAD3 ATF5 BCL2L1 MCL1 BCL2 } \\
\text { CD9 IKBKB ANPEP SYNCRIP PTBP1 CEACAM1 ANXA2 HSPA9 GSK3A GSK3B } \\
\text { PABPC4 NPM1 PARP1 BAG6 H2AFY2 CHEK2 DDX5 CAV1 }\end{array}$ \\
\hline GO:0050794 & regulation of cellular process & $86 / 119$ & $\begin{array}{l}\text { SCFD1 PSMC2 TFEB TWF2 KPNB1 STX5 STAT5A PHB JUN STAT3 PPP1CA XPO1 } \\
\text { TGFB1 SMAD3 ATF5 COX2 HNRNPA1 ACE2 CLEC4G CD209 CLEC4M IRF3 KPNA2 } \\
\text { SFTPD PPIA BCL2L1 BCL2L2 MCL1 BCL2A1 BCL2 SKP2 PRKRA CD9 IKBKB DPP4 } \\
\text { SYNCRIP PTBP1 CEACAM1 ANXA2 HNRNPA2B1 DNAJB1 HSPD1 HSPA9 RPL13A } \\
\text { EIF3E NMT1 CHMP4B GBF1 RRM2 KIF11 SNX9 NONO GSK3A GSK3B PABPC1 } \\
\text { PABPC4 NPM1 G3BP1 G3BP2 PARP1 NCL DDX1 RYBP FKBP1A MARK3 RCAN3 } \\
\text { HGS BAG6 DDAH2 CAMLG CHMP2B SNAP47 SERPING1 ABHD17A PFDN5 } \\
\text { MIF4GD H2AFY2 CHEK2 TERF1 DCTN2 DDX5 EEF1A1 CAV1 UBE2I SGTA } \\
\text { ATP6V1G1 }\end{array}$ \\
\hline GO:0051301 & cell division & $10 / 119$ & PPP1CA TGFB1 BCL2L1 CHMP4B KIF11 SNX9 CHMP2B CHEK2 TERF1 UBE2I \\
\hline GO:0051651 & maintenance of location in cell & $2 / 119$ & TWF2 CAV1 \\
\hline GO:0051716 & cellular response to stimulus & $60 / 119$ & $\begin{array}{l}\text { TWF2 KPNB1 STAT5A PHB JUN STAT3 PPP1CA TGFB1 SMAD3 HNRNPA1 CD209 } \\
\text { CLEC4M IRF3 KPNA2 SFTPD PPIA BCL2L1 BCL2L2 MCL1 BCL2A1 BCL2 SKP2 } \\
\text { PRKRA CD9 IKBKB SYNCRIP PTBP1 CEACAM1 ANXA2 HNRNPA2B1 DNAJB1 } \\
\text { HSPD1 HSPA9 RPL13A NMT1 GBF1 NONO GSK3A GSK3B NPM1 G3BP1 G3BP2 } \\
\text { PARP1 NCL DDX1 FKBP1A MARK3 RCAN3 HGS BAG6 DDAH2 CAMLG PFDN5 } \\
\text { CHEK2 DDX5 EEF1A1 CAV1 UBE2I SGTA ATP6V1G1 }\end{array}$ \\
\hline GO:0060352 & $\begin{array}{l}\text { cell adhesion molecule } \\
\text { production }\end{array}$ & $1 / 119$ & CAV1 \\
\hline GO:0061919 & $\begin{array}{l}\text { process utilizing autophagic } \\
\text { mechanism }\end{array}$ & $11 / 119$ & $\begin{array}{l}\text { SCFD1 TFEB ACBD5 MCL1 BCL2 CHMP4B GSK3A HGS CHMP2B EEF1A1 } \\
\text { ATP6V1G1 }\end{array}$ \\
\hline GO:0071804 & cellular potassium ion transport & $1 / 119$ & CAV1 \\
\hline GO:0097194 & execution phase of apoptosis & $2 / 119$ & KPNB1 BCL2L1 \\
\hline GO:0140029 & exocytic process & $3 / 119$ & SCFD1 YKT6 SNAP47 \\
\hline GO:0006808 & regulation of nitrogen utilization & $1 / 119$ & BCL2 \\
\hline GO:0003006 & $\begin{array}{l}\text { developmental process involved } \\
\text { in reproduction }\end{array}$ & $6 / 119$ & TFEB PHB TGFB1 BCL2L1 BCL2L2 BCL2 \\
\hline GO:0009566 & fertilization & $2 / 119$ & BCL2L1 CD9 \\
\hline GO:0044703 & $\begin{array}{l}\text { multi-organism reproductive } \\
\text { process }\end{array}$ & $7 / 119$ & STAT3 TGFB1 BCL2L1 BCL2L2 BCL2 CD9 BAG6 \\
\hline GO:0048609 & $\begin{array}{l}\text { multicellular organismal } \\
\text { reproductive process }\end{array}$ & $5 / 119$ & TGFB1 BCL2L1 BCL2L2 BCL2 BAG6 \\
\hline GO:0051321 & meiotic cell cycle & $2 / 119$ & BAG6 TERF1 \\
\hline GO:0060011 & Sertoli cell proliferation & $1 / 119$ & BCL2L2 \\
\hline GO:0090220 & $\begin{array}{l}\text { chromosome localization to } \\
\text { nuclear envelope involved in } \\
\text { homologous chromosome } \\
\text { segregation }\end{array}$ & $1 / 119$ & TERF1 \\
\hline GO:1903046 & meiotic cell cycle process & $2 / 119$ & BAG6 TERF1 \\
\hline
\end{tabular}




\begin{tabular}{|c|c|c|c|}
\hline ID & Description & GeneRatio & geneID \\
\hline GO:0007155 & cell adhesion & $15 / 119$ & $\begin{array}{l}\text { PPP1CA TGFB1 SMAD3 CLEC4G CD209 CLEC4M SFTPD BCL2 CD9 DPP4 } \\
\text { CEACAM1 ANXA2 HSPD1 GSK3B CAV1 }\end{array}$ \\
\hline GO:0044406 & adhesion of symbiont to host & $4 / 119$ & ACE2 CD209 CLEC4M SFTPD \\
\hline GO:0007267 & cell-cell signaling & $11 / 119$ & STAT3 PPP1CA SMAD3 KPNA2 DPP4 GSK3A GSK3B G3BP1 SNAP47 PFDN5 CAV1 \\
\hline GO:0023051 & regulation of signaling & $39 / 119$ & $\begin{array}{c}\text { PHB JUN STAT3 PPP1CA TGFB1 SMAD3 ACE2 IRF3 BCL2L1 BCL2L2 MCL1 } \\
\text { BCL2A1 BCL2 SKP2 PRKRA IKBKB DPP4 PTBP1 CEACAM1 ANXA2 NMT1 GBF1 } \\
\text { NONO GSK3A GSK3B G3BP1 PARP1 DDX1 FKBP1A MARK3 RCAN3 HGS CAMLG } \\
\text { SNAP47 PFDN5 CHEK2 DDX5 CAV1 UBE2I }\end{array}$ \\
\hline GO:0023056 & positive regulation of signaling & $25 / 119$ & $\begin{array}{l}\text { PHB JUN STAT3 PPP1CA TGFB1 SMAD3 IRF3 BCL2L1 MCL1 BCL2 SKP2 PRKRA } \\
\text { IKBKB PTBP1 ANXA2 NMT1 GSK3A GSK3B PARP1 DDX1 FKBP1A SNAP47 DDX5 } \\
\text { CAV1 UBE2I }\end{array}$ \\
\hline GO:0023057 & negative regulation of signaling & $17 / 119$ & $\begin{array}{l}\text { PHB TGFB1 SMAD3 BCL2L1 BCL2L2 MCL1 BCL2A1 BCL2 CEACAM1 NONO } \\
\text { GSK3A GSK3B G3BP1 MARK3 HGS PFDN5 CAV1 }\end{array}$ \\
\hline GO:0035637 & $\begin{array}{l}\text { multicellular organismal } \\
\text { signaling }\end{array}$ & $2 / 119$ & ACE2 CAV1 \\
\hline GO:0001503 & ossification & $7 / 119$ & PSMC2 PHB TGFB1 SMAD3 BCL2 SYNCRIP DDX5 \\
\hline GO:0001763 & $\begin{array}{l}\text { morphogenesis of a branching } \\
\text { structure }\end{array}$ & $3 / 119$ & PPP1CA TGFB1 BCL2 \\
\hline GO:0001816 & cytokine production & $8 / 119$ & TGFB1 SMAD3 ACE2 IRF3 SFTPD CEACAM1 HSPD1 DDX1 \\
\hline GO:0003008 & system process & $12 / 119$ & $\begin{array}{l}\text { JUN TGFB1 SMAD3 ACE2 BCL2 CEACAM1 GSK3A GSK3B PARP1 CHMP2B } \\
\text { SERPING1 CAV1 }\end{array}$ \\
\hline GO:0007275 & $\begin{array}{l}\text { multicellular organism } \\
\text { development }\end{array}$ & $44 / 119$ & $\begin{array}{l}\text { TFEB TWF2 STAT5A PHB JUN STAT3 PPP1CA TGFB1 SMAD3 ATF5 COX2 SFTPD } \\
\text { BCL2L1 BCL2L2 MCL1 BCL2A1 BCL2 PRKRA CD9 IKBKB ANPEP CEACAM1 } \\
\text { ANXA2 ACO2 DNAJB1 HSPD1 HSPA9 NMT1 GSK3A GSK3B PABPC4 RPL19 PARP1 } \\
\text { NCL DDX1 RYBP FKBP1A HGS BAG6 PFDN5 VKORC1 H2AFY2 DDX5 CAV1 }\end{array}$ \\
\hline GO:0007389 & pattern specification process & $1 / 119$ & SMAD3 \\
\hline GO:0007585 & respiratory gaseous exchange & $1 / 119$ & SFTPD \\
\hline GO:0009791 & post-embryonic development & $2 / 119$ & ATF5 BCL2 \\
\hline GO:0019827 & stem cell population maintenance & $1 / 119$ & STAT3 \\
\hline GO:0022404 & molting cycle process & $1 / 119$ & BCL2 \\
\hline GO:0032922 & $\begin{array}{l}\text { circadian regulation of gene } \\
\text { expression }\end{array}$ & $1 / 119$ & PPP1CA \\
\hline GO:0033555 & $\begin{array}{l}\text { multicellular organismal } \\
\text { response to stress }\end{array}$ & $2 / 119$ & BCL2 DPP4 \\
\hline GO:0034381 & $\begin{array}{l}\text { plasma lipoprotein particle } \\
\text { clearance }\end{array}$ & $1 / 119$ & ANXA2 \\
\hline GO:0035264 & multicellular organism growth & $4 / 119$ & STAT5A STAT3 ATF5 BCL2 \\
\hline GO:0035265 & organ growth & $3 / 119$ & BCL2 ANXA2 GSK3A \\
\hline GO:0042303 & molting cycle & $1 / 119$ & BCL2 \\
\hline GO:0044706 & $\begin{array}{l}\text { multi-multicellular organism } \\
\text { process }\end{array}$ & $2 / 119$ & TGFB1 BCL2 \\
\hline
\end{tabular}




\begin{tabular}{|c|c|c|c|}
\hline ID & Description & GeneRatio & geneID \\
\hline GO:0048771 & tissue remodeling & $3 / 119$ & TGFB1 CEACAM1 CAV1 \\
\hline GO:0048871 & $\begin{array}{l}\text { multicellular organismal } \\
\text { homeostasis }\end{array}$ & $5 / 119$ & STAT3 SFTPD BCL2 CHMP4B CAV1 \\
\hline GO:0050817 & coagulation & $7 / 119$ & CD9 CEACAM1 ANXA2 PABPC4 SERPING1 VKORC1 CAV1 \\
\hline GO:0051239 & $\begin{array}{l}\text { regulation of multicellular } \\
\text { organismal process }\end{array}$ & $27 / 119$ & $\begin{array}{c}\text { TWF2 STAT5A JUN STAT3 TGFB1 SMAD3 ACE2 IRF3 SFTPD BCL2 CD9 IKBKB } \\
\text { CEACAM1 ANXA2 HSPD1 HSPA9 GSK3A GSK3B PARP1 DDX1 HGS BAG6 } \\
\text { SERPING1 VKORC1 H2AFY2 DDX5 CAV1 }\end{array}$ \\
\hline GO:0051240 & $\begin{array}{l}\text { positive regulation of } \\
\text { multicellular organismal process }\end{array}$ & $17 / 119$ & $\begin{array}{c}\text { TWF2 STAT5A JUN STAT3 TGFB1 SMAD3 ACE2 IRF3 BCL2 CEACAM1 ANXA2 } \\
\text { HSPD1 GSK3A PARP1 DDX1 H2AFY2 CAV1 }\end{array}$ \\
\hline GO:0051241 & $\begin{array}{l}\text { negative regulation of } \\
\text { multicellular organismal process }\end{array}$ & $14 / 119$ & $\begin{array}{l}\text { STAT3 TGFB1 SMAD3 IRF3 SFTPD BCL2 CD9 CEACAM1 ANXA2 HSPA9 GSK3A } \\
\text { GSK3B HGS SERPING1 }\end{array}$ \\
\hline GO:0090130 & tissue migration & $5 / 119$ & STAT5A JUN TGFB1 DPP4 CEACAM1 \\
\hline GO:0009653 & $\begin{array}{l}\text { anatomical structure } \\
\text { morphogenesis }\end{array}$ & $21 / 119$ & $\begin{array}{l}\text { SCFD1 TWF2 JUN STAT3 PPP1CA TGFB1 SMAD3 BCL2L1 BCL2 PRKRA CD9 } \\
\text { ANPEP CEACAM1 ANXA2 GSK3A GSK3B NCL FKBP1A RCAN3 HGS CAV1 }\end{array}$ \\
\hline GO:0007568 & aging & $8 / 119$ & JUN STAT3 TGFB1 BCL2A1 BCL2 NPM1 SERPING1 CHEK2 \\
\hline GO:0048646 & $\begin{array}{l}\text { anatomical structure formation } \\
\text { involved in morphogenesis }\end{array}$ & $12 / 119$ & $\begin{array}{c}\text { JUN STAT3 TGFB1 SMAD3 CD9 ANPEP CEACAM1 ANXA2 NCL FKBP1A HGS } \\
\text { CAV1 }\end{array}$ \\
\hline GO:0021700 & developmental maturation & $1 / 119$ & BCL2 \\
\hline GO:0044111 & $\begin{array}{l}\text { development involved in } \\
\text { symbiotic interaction }\end{array}$ & $1 / 119$ & ANXA2 \\
\hline GO:0048856 & $\begin{array}{l}\text { anatomical structure } \\
\text { development }\end{array}$ & $47 / 119$ & $\begin{array}{c}\text { SCFD1 TFEB TWF2 STAT5A PHB JUN STAT3 PPP1CA TGFB1 SMAD3 ATF5 COX2 } \\
\text { SFTPD BCL2L1 BCL2L2 MCL1 BCL2A1 BCL2 PRKRA CD9 IKBKB ANPEP PTBP1 } \\
\text { CEACAM1 ANXA2 ACO2 DNAJB1 HSPD1 HSPA9 NMT1 GSK3A GSK3B PABPC4 } \\
\text { RPL19 PARP1 NCL DDX1 RYBP FKBP1A RCAN3 HGS BAG6 PFDN5 VKORC1 } \\
\text { H2AFY2 DDX5 CAV1 }\end{array}$ \\
\hline GO:0050793 & $\begin{array}{l}\text { regulation of developmental } \\
\text { process }\end{array}$ & $20 / 119$ & $\begin{array}{l}\text { TWF2 STAT5A JUN STAT3 TGFB1 SMAD3 BCL2 IKBKB PTBP1 CEACAM1 ANXA2 } \\
\text { HSPA9 GSK3A GSK3B PARP1 HGS BAG6 H2AFY2 DDX5 CAV1 }\end{array}$ \\
\hline GO:0051093 & $\begin{array}{l}\text { negative regulation of } \\
\text { developmental process }\end{array}$ & $12 / 119$ & $\begin{array}{l}\text { STAT3 TGFB1 SMAD3 BCL2 PTBP1 CEACAM1 ANXA2 HSPA9 GSK3A GSK3B HGS } \\
\text { CAV1 }\end{array}$ \\
\hline GO:0051094 & $\begin{array}{l}\text { positive regulation of } \\
\text { developmental process }\end{array}$ & $10 / 119$ & TWF2 JUN STAT3 TGFB1 SMAD3 BCL2 CEACAM1 ANXA2 PARP1 H2AFY2 \\
\hline GO:0098727 & maintenance of cell number & $1 / 119$ & STAT3 \\
\hline GO:0040012 & regulation of locomotion & $10 / 119$ & STAT5A JUN STAT3 TGFB1 SMAD3 BCL2 CEACAM1 CHMP4B CHMP2B CAV1 \\
\hline GO:0040013 & $\begin{array}{l}\text { negative regulation of } \\
\text { locomotion }\end{array}$ & $3 / 119$ & STAT3 TGFB1 BCL2 \\
\hline GO:0040017 & positive regulation of locomotion & $9 / 119$ & STAT5A JUN STAT3 TGFB1 SMAD3 BCL2 CHMP4B CHMP2B CAV1 \\
\hline GO:0042330 & taxis & $4 / 119$ & TGFB1 SMAD3 SFTPD GBF1 \\
\hline GO:0048870 & cell motility & $12 / 119$ & $\begin{array}{l}\text { STAT5A JUN STAT3 TGFB1 SMAD3 SFTPD PPIA BCL2 DPP4 CEACAM1 GBF1 } \\
\text { CAV1 }\end{array}$ \\
\hline GO:0052192 & $\begin{array}{l}\text { movement in environment of } \\
\text { other organism involved in } \\
\text { symbiotic interaction }\end{array}$ & $3 / 119$ & PPIA CHMP4B CHMP2B \\
\hline
\end{tabular}




\begin{tabular}{|c|c|c|c|}
\hline ID & Description & GeneRatio & geneID \\
\hline GO:0048066 & developmental pigmentation & $1 / 119$ & BCL2 \\
\hline GO:0007623 & circadian rhythm & $5 / 119$ & JUN PPP1CA ATF5 NONO GSK3B \\
\hline GO:0043902 & $\begin{array}{l}\text { positive regulation of multi- } \\
\text { organism process }\end{array}$ & $8 / 119$ & JUN PPIA TMPRSS2 ANXA2 CHMP4B PABPC1 PPIH CHMP2B \\
\hline GO:0044089 & $\begin{array}{l}\text { positive regulation of cellular } \\
\text { component biogenesis }\end{array}$ & $12 / 119$ & PSMC2 TWF2 JUN TGFB1 SMAD3 ACE2 SNX9 GSK3B PARP1 HGS TERF1 CAV1 \\
\hline GO:0045785 & $\begin{array}{l}\text { positive regulation of cell } \\
\text { adhesion }\end{array}$ & $7 / 119$ & TGFB1 SMAD3 CD209 DPP4 HSPD1 GSK3B CAV1 \\
\hline GO:0048087 & $\begin{array}{c}\text { positive regulation of } \\
\text { developmental pigmentation }\end{array}$ & $1 / 119$ & BCL2 \\
\hline GO:0048584 & $\begin{array}{c}\text { positive regulation of response to } \\
\text { stimulus }\end{array}$ & $31 / 119$ & $\begin{array}{l}\text { PHB JUN STAT3 PPP1CA TGFB1 SMAD3 CD209 IRF3 SFTPD BCL2L1 MCL1 BCL2 } \\
\text { SKP2 PRKRA IKBKB PTBP1 CEACAM1 HSPD1 NMT1 NONO GSK3A GSK3B PARP1 } \\
\text { DDX1 FKBP1A BAG6 SERPING1 DDX5 CAV1 UBE2I SGTA }\end{array}$ \\
\hline GO:0051050 & positive regulation of transport & $14 / 119$ & $\begin{array}{l}\text { TGFB1 SMAD3 ACE2 IRF3 SFTPD PPIA BCL2 ANXA2 HSPD1 NMT1 GSK3A GSK3B } \\
\text { HGS CAV1 }\end{array}$ \\
\hline GO:0051091 & $\begin{array}{l}\text { positive regulation of DNA- } \\
\text { binding transcription factor } \\
\text { activity }\end{array}$ & $5 / 119$ & TGFB1 SMAD3 IKBKB NPM1 UBE2I \\
\hline GO: 1903829 & $\begin{array}{l}\text { positive regulation of cellular } \\
\text { protein localization }\end{array}$ & $8 / 119$ & TGFB1 SMAD3 BCL2 NMT1 GSK3A GSK3B PARP1 ABHD17A \\
\hline GO:1904181 & $\begin{array}{l}\text { positive regulation of membrane } \\
\text { depolarization }\end{array}$ & $1 / 119$ & PARP1 \\
\hline GO:1904951 & $\begin{array}{l}\text { positive regulation of } \\
\text { establishment of protein } \\
\text { localization }\end{array}$ & $9 / 119$ & TGFB1 SMAD3 IRF3 PPIA BCL2 HSPD1 NMT1 GSK3A GSK3B \\
\hline GO:1905954 & $\begin{array}{l}\text { positive regulation of lipid } \\
\text { localization }\end{array}$ & $1 / 119$ & ANXA2 \\
\hline GO:0043433 & $\begin{array}{l}\text { negative regulation of DNA- } \\
\text { binding transcription factor } \\
\text { activity }\end{array}$ & $1 / 119$ & G3BP2 \\
\hline GO:0043901 & $\begin{array}{l}\text { negative regulation of multi- } \\
\text { organism process }\end{array}$ & $2 / 119$ & JUN ANXA2 \\
\hline GO:0048585 & $\begin{array}{l}\text { negative regulation of response } \\
\text { to stimulus }\end{array}$ & $23 / 119$ & $\begin{array}{l}\text { PHB TGFB1 SMAD3 CLEC4G BCL2L1 BCL2L2 MCL1 BCL2A1 BCL2 CD9 } \\
\text { CEACAM1 ANXA2 NONO GSK3A GSK3B G3BP1 MARK3 HGS SERPING1 PFDN5 } \\
\text { CHEK2 CAV1 SGTA }\end{array}$ \\
\hline GO:0051051 & negative regulation of transport & $5 / 119$ & TGFB1 BCL2 CEACAM1 GSK3A CAV1 \\
\hline GO: 1900047 & negative regulation of hemostasis & $4 / 119$ & CD9 CEACAM1 ANXA2 SERPING1 \\
\hline GO: 1903828 & $\begin{array}{l}\text { negative regulation of cellular } \\
\text { protein localization }\end{array}$ & $5 / 119$ & TGFB1 BCL2L1 GSK3B ABHD17A TERF1 \\
\hline GO: 1904180 & $\begin{array}{l}\text { negative regulation of membrane } \\
\text { depolarization }\end{array}$ & $1 / 119$ & BCL2 \\
\hline GO:1904911 & $\begin{array}{l}\text { negative regulation of } \\
\text { establishment of RNA } \\
\text { localization to telomere }\end{array}$ & $1 / 119$ & TERF1 \\
\hline
\end{tabular}




\begin{tabular}{|c|c|c|c|}
\hline ID & Description & GeneRatio & geneID \\
\hline GO:1904914 & $\begin{array}{l}\text { negative regulation of } \\
\text { establishment of protein- } \\
\text { containing complex localization } \\
\text { to telomere }\end{array}$ & $1 / 119$ & TERF1 \\
\hline GO: 1904950 & $\begin{array}{l}\text { negative regulation of } \\
\text { establishment of protein } \\
\text { localization }\end{array}$ & $1 / 119$ & TERF1 \\
\hline GO:0030155 & regulation of cell adhesion & $12 / 119$ & $\begin{array}{l}\text { TGFB1 SMAD3 CLEC4G CD209 SFTPD BCL2 CD9 DPP4 CEACAM1 HSPD1 GSK3B } \\
\text { CAV1 }\end{array}$ \\
\hline GO:0032879 & regulation of localization & $28 / 119$ & $\begin{array}{c}\text { SCFD1 STAT5A JUN STAT3 XPO1 TGFB1 SMAD3 ACE2 IRF3 SFTPD PPIA BCL2L1 } \\
\text { BCL2 CD9 DPP4 CEACAM1 ANXA2 HSPD1 NMT1 GBF1 GSK3A GSK3B PARP1 } \\
\text { FKBP1A HGS ABHD17A TERF1 CAV1 }\end{array}$ \\
\hline GO:0042752 & regulation of circadian rhythm & $2 / 119$ & PPP1CA NONO \\
\hline GO:0043900 & $\begin{array}{l}\text { regulation of multi-organism } \\
\text { process }\end{array}$ & $12 / 119$ & $\begin{array}{l}\text { JUN SFTPD PPIA BCL2 TMPRSS2 ANXA2 CHMP4B PABPC1 PPIH CHMP2B DDX5 } \\
\text { CAV1 }\end{array}$ \\
\hline GO:0044087 & $\begin{array}{l}\text { regulation of cellular component } \\
\text { biogenesis }\end{array}$ & $19 / 119$ & $\begin{array}{l}\text { SCFD1 PSMC2 TWF2 JUN TGFB1 SMAD3 ACE2 IKBKB DNAJB1 RPL13A CHMP4B } \\
\text { SNX9 GSK3B NPM1 PARP1 HGS CHMP2B TERF1 CAV1 }\end{array}$ \\
\hline GO:0048070 & $\begin{array}{l}\text { regulation of developmental } \\
\text { pigmentation }\end{array}$ & $1 / 119$ & BCL2 \\
\hline GO:0048518 & $\begin{array}{l}\text { positive regulation of biological } \\
\text { process }\end{array}$ & $63 / 119$ & $\begin{array}{l}\text { PSMC2 TFEB TWF2 STX5 STAT5A PHB JUN STAT3 PPP1CA TGFB1 SMAD3 ATF5 } \\
\text { COX2 HNRNPA1 ACE2 CD209 IRF3 SFTPD PPIA BCL2L1 MCL1 BCL2 SKP2 PRKRA } \\
\text { TMPRSS2 IKBKB DPP4 SYNCRIP PTBP1 CEACAM1 ANXA2 HNRNPA2B1 HSPD1 } \\
\text { EIF3E NMT1 CHMP4B SNX9 NONO GSK3A GSK3B PABPC1 NPM1 PARP1 NCL } \\
\text { DDX1 RYBP FKBP1A PPIH HGS BAG6 DDAH2 CHMP2B SNAP47 SERPING1 } \\
\text { ABHD17A MIF4GD H2AFY2 CHEK2 TERF1 DDX5 CAV1 UBE2I SGTA }\end{array}$ \\
\hline GO:0048519 & $\begin{array}{l}\text { negative regulation of biological } \\
\text { process }\end{array}$ & $62 / 119$ & $\begin{array}{l}\text { SCFD1 TWF2 PHB JUN STAT3 XPO1 TGFB1 SMAD3 ATF5 HNRNPA1 CLEC4G IRF3 } \\
\text { SFTPD BCL2L1 BCL2L2 MCL1 BCL2A1 BCL2 PRKRA CD9 IKBKB DPP4 SYNCRIP } \\
\text { PTBP1 CEACAM1 ANXA2 HNRNPA2B1 DNAJB1 HSPD1 HSPA9 RPL13A EIF3E } \\
\text { CHMP4B RRM2 NONO GSK3A GSK3B PABPC1 PABPC4 NPM1 G3BP1 G3BP2 } \\
\text { RPL19 PARP1 NCL RYBP FKBP1A MARK3 HGS BAG6 DDAH2 SERPING1 } \\
\text { ABHD17A PFDN5 H2AFY2 RPS20 CHEK2 TERF1 DDX5 CAV1 UBE2I SGTA }\end{array}$ \\
\hline GO:0048583 & $\begin{array}{l}\text { regulation of response to } \\
\text { stimulus }\end{array}$ & $47 / 119$ & $\begin{array}{c}\text { PHB JUN STAT3 PPP1CA TGFB1 SMAD3 ACE2 CLEC4G CD209 IRF3 SFTPD } \\
\text { BCL2L1 BCL2L2 MCL1 BCL2A1 BCL2 SKP2 PRKRA CD9 IKBKB PTBP1 CEACAM1 } \\
\text { ANXA2 DNAJB1 HSPD1 NMT1 GBF1 NONO GSK3A GSK3B NPM1 G3BP1 PARP1 } \\
\text { DDX1 FKBP1A MARK3 RCAN3 HGS BAG6 SERPING1 PFDN5 VKORC1 CHEK2 } \\
\text { DDX5 CAV1 UBE2I SGTA }\end{array}$ \\
\hline GO:0097006 & $\begin{array}{l}\text { regulation of plasma lipoprotein } \\
\text { particle levels }\end{array}$ & $1 / 119$ & ANXA2 \\
\hline GO:0098900 & regulation of action potential & $1 / 119$ & CAV1 \\
\hline GO:1900046 & regulation of hemostasis & $6 / 119$ & CD9 CEACAM1 ANXA2 SERPING1 VKORC1 CAV1 \\
\hline GO:0006950 & response to stress & $49 / 119$ & $\begin{array}{l}\text { SCFD1 PHB JUN STAT3 PPP1CA TGFB1 SMAD3 COX2 HNRNPA1 ACE2 CD209 } \\
\text { CLEC4M IRF3 SFTPD BCL2L1 BCL2L2 MCL1 BCL2A1 BCL2 SKP2 KPNA4 PRKRA } \\
\text { CD9 IKBKB DPP4 SYNCRIP CEACAM1 ANXA2 ACO2 DNAJB1 HSPD1 HSPA9 } \\
\text { RPL13A NONO GSK3A GSK3B PABPC4 NPM1 PARP1 DDX1 BAG6 CAMLG } \\
\text { TPSAB1 SERPING1 VKORC1 CHEK2 DDX5 CAV1 SGTA }\end{array}$ \\
\hline GO:0009605 & response to external stimulus & $25 / 119$ & $\begin{array}{c}\text { PHB JUN PPP1CA TGFB1 SMAD3 HNRNPA1 ACE2 IRF3 SFTPD BCL2L1 BCL2 SKP2 } \\
\text { PRKRA CD9 IKBKB CEACAM1 ANXA2 HSPD1 NMT1 GBF1 DDX1 SERPING1 } \\
\text { PSMA2 VKORC1 CAV1 }\end{array}$ \\
\hline GO:0009607 & response to biotic stimulus & $17 / 119$ & $\begin{array}{l}\text { JUN TGFB1 SMAD3 IRF3 SFTPD BCL2L1 BCL2 SKP2 PRKRA IKBKB CEACAM1 } \\
\text { HSPD1 GBF1 GSK3B DDX1 PSMA2 CAV1 }\end{array}$ \\
\hline GO:0009628 & response to abiotic stimulus & $20 / 119$ & $\begin{array}{l}\text { SCFD1 JUN PPP1CA TGFB1 SMAD3 COX2 BCL2L1 BCL2 CD9 DPP4 DNAJB1 } \\
\text { HSPD1 HSPA9 NMT1 GSK3B NPM1 PARP1 CHEK2 CAV1 ATP6V1G1 }\end{array}$ \\
\hline
\end{tabular}




\begin{tabular}{|c|c|c|c|}
\hline ID & Description & GeneRatio & geneID \\
\hline GO:0009719 & response to endogenous stimulus & $25 / 119$ & $\begin{array}{c}\text { STAT5A PHB JUN STAT3 TGFB1 SMAD3 HNRNPA1 BCL2L1 BCL2 SKP2 CD9 } \\
\text { PTBP1 CEACAM1 ANXA2 HSPD1 GSK3A GSK3B PARP1 NCL CHEK2 DDX5 } \\
\text { EEF1A1 CAV1 UBE2I ATP6V1G1 }\end{array}$ \\
\hline GO:0014823 & response to activity & $1 / 119$ & HSPD1 \\
\hline GO:0042221 & response to chemical & $51 / 119$ & $\begin{array}{l}\text { SCFD1 TWF2 STAT5A PHB JUN STAT3 PPP1CA XPO1 TGFB1 SMAD3 HNRNPA1 } \\
\text { IRF3 SFTPD PPIA BCL2L1 MCL1 BCL2 SKP2 KPNA4 PRKRA CD9 IKBKB SYNCRIP } \\
\text { PTBP1 CEACAM1 ANXA2 HNRNPA2B1 DNAJB1 HSPD1 HSPA9 RPL13A GBF1 } \\
\text { SRP54 NONO GSK3A GSK3B NPM1 PARP1 NCL DDX1 HGS BAG6 VKORC1 CHEK2 } \\
\text { TERF1 DDX5 EEF1A1 CAV1 UBE2I SGTA ATP6V1G1 }\end{array}$ \\
\hline GO:0043500 & muscle adaptation & $3 / 119$ & SMAD3 GSK3A PARP1 \\
\hline GO:0051606 & detection of stimulus & $2 / 119$ & NMT1 PARP1 \\
\hline GO:0072376 & protein activation cascade & $2 / 119$ & PHB SERPING1 \\
\hline GO:0031503 & $\begin{array}{l}\text { protein-containing complex } \\
\text { localization }\end{array}$ & $5 / 119$ & XPO1 HNRNPA2B1 NPM1 SNAP47 TERF1 \\
\hline GO:0033036 & macromolecule localization & $52 / 119$ & $\begin{array}{l}\text { BTF3 SCFD1 TWF2 YKT6 KPNB1 STX5 STAT3 XPO1 TGFB1 SMAD3 HNRNPA1 } \\
\text { IRF3 KPNA2 PPIA BCL2L1 MCL1 BCL2 KPNA4 IKBKB DPP4 CEACAM1 ANXA2 } \\
\text { HNRNPA2B1 HNRNPA3 HSPD1 COPB2 RPL13A NMT1 CHMP4B GBF1 SRP54 SNX9 } \\
\text { GSK3A GSK3B NPM1 G3BP2 RPL19 PARP1 DDX1 FKBP1A HGS BAG6 CHMP2B } \\
\text { SNAP47 ABHD17A H2AFY2 RPS20 TERF1 DCTN2 CAV1 SGTA ATP6V1G1 }\end{array}$ \\
\hline GO:0051234 & establishment of localization & $64 / 119$ & $\begin{array}{l}\text { BTF3 SCFD1 PSMC2 YKT6 KPNB1 STX5 STAT5A PHB STAT3 FGL2 XPO1 TGFB1 } \\
\text { SMAD3 COX2 HNRNPA1 ACE2 CD209 CLEC4M IRF3 KPNA2 SFTPD PPIA BCL2L1 } \\
\text { MCL1 BCL2 KPNA4 CD9 TMPRSS2 ANPEP DPP4 CEACAM1 ANXA2 HNRNPA2B1 } \\
\text { HNRNPA3 HSPD1 COPB2 RPL13A NMT1 CHMP4B GBF1 KIF11 PSMD1 SRP54 } \\
\text { SNX9 GSK3A GSK3B NPM1 G3BP2 RPL19 FKBP1A HGS BAG6 CHMP2B SNAP47 } \\
\text { SERPING1 PSMA2 H2AFY2 RPS20 TERF1 DCTN2 EEF1A1 CAV1 SGTA ATP6V1G1 }\end{array}$ \\
\hline GO:0051235 & maintenance of location & $5 / 119$ & TWF2 TGFB1 G3BP2 FKBP1A CAV1 \\
\hline GO:0051641 & cellular localization & $43 / 119$ & $\begin{array}{c}\text { SCFD1 TWF2 YKT6 KPNB1 STX5 STAT3 XPO1 TGFB1 SMAD3 HNRNPA1 KPNA2 } \\
\text { BCL2L1 BCL2 KPNA4 IKBKB ANXA2 HNRNPA2B1 HSPD1 COPB2 RPL13A NMT1 } \\
\text { CHMP4B GBF1 SRP54 SNX9 GSK3A GSK3B NPM1 RPL19 PARP1 DDX1 FKBP1A } \\
\text { HGS BAG6 CHMP2B SNAP47 ABHD17A H2AFY2 RPS20 TERF1 DCTN2 CAV1 } \\
\text { SGTA }\end{array}$ \\
\hline GO:0051674 & localization of cell & $12 / 119$ & $\begin{array}{l}\text { STAT5A JUN STAT3 TGFB1 SMAD3 SFTPD PPIA BCL2 DPP4 CEACAM1 GBF1 } \\
\text { CAV1 }\end{array}$ \\
\hline GO:1902579 & multi-organism localization & $5 / 119$ & KPNB1 XPO1 CD209 CLEC4M CAV1 \\
\hline GO:0051707 & response to other organism & $15 / 119$ & $\begin{array}{l}\text { JUN TGFB1 SMAD3 IRF3 SFTPD BCL2L1 BCL2 SKP2 PRKRA IKBKB HSPD1 GBF1 } \\
\text { DDX1 PSMA2 CAV1 }\end{array}$ \\
\hline GO:0044419 & $\begin{array}{l}\text { interspecies interaction between } \\
\text { organisms }\end{array}$ & $38 / 119$ & $\begin{array}{c}\text { KPNB1 JUN STAT3 XPO1 TGFB1 SMAD3 HNRNPA1 ACE2 CLEC4G CD209 } \\
\text { CLEC4M IRF3 KPNA2 SFTPD PPIA BCL2L1 BCL2 KPNA4 TMPRSS2 IKBKB ANPEP } \\
\text { DPP4 SYNCRIP PTBP1 ANXA2 HSPD1 CHMP4B EIF3F GBF1 PABPC1 NPM1 PPIH } \\
\text { CAMLG CHMP2B DDX5 CAV1 UBE2I SGTA }\end{array}$ \\
\hline GO:0050789 & regulation of biological process & $92 / 119$ & $\begin{array}{l}\text { SCFD1 PSMC2 TFEB TWF2 KPNB1 STX5 STAT5A PHB JUN STAT3 PPP1CA XPO1 } \\
\text { TGFB1 SMAD3 ATF5 COX2 HNRNPA1 ACE2 CLEC4G CD209 CLEC4M IRF3 KPNA2 } \\
\text { SFTPD PPIA BCL2L1 BCL2L2 MCL1 BCL2A1 BCL2 SKP2 PRKRA CD9 TMPRSS2 } \\
\text { IKBKB DPP4 SYNCRIP PTBP1 CEACAM1 ANXA2 HNRNPA2B1 DNAJB1 HSPD1 } \\
\text { HSPA9 RPL13A EIF3E NMT1 CHMP4B GBF1 RRM2 KIF11 PSMD1 SNX9 NONO } \\
\text { GSK3A GSK3B PABPC1 PABPC4 NPM1 G3BP1 G3BP2 RPL19 PARP1 NCL DDX1 } \\
\text { RYBP FKBP1A MARK3 PPIH RCAN3 HGS BAG6 DDAH2 CAMLG CHMP2B SNAP47 } \\
\text { SERPING1 ABHD17A PFDN5 MIF4GD VKORC1 H2AFY2 RPS20 CHEK2 TERF1 } \\
\text { DCTN2 DDX5 EEF1A1 CAV1 UBE2I SGTA ATP6V1G1 }\end{array}$ \\
\hline
\end{tabular}




\begin{tabular}{|c|c|c|c|}
\hline ID & Description & GeneRatio & geneID \\
\hline & & & $\begin{array}{l}\text { NPM1 G3BP2 PARP1 FKBP1A BAG6 DDAH2 CHMP2B SNAP47 SERPING1 } \\
\text { ABHD17A VKORC1 CHEK2 TERF1 CAV1 ATP6V1G1 }\end{array}$ \\
\hline GO:0065009 & regulation of molecular function & $31 / 119$ & $\begin{array}{c}\text { JUN STAT3 PPP1CA TGFB1 SMAD3 SFTPD BCL2 PRKRA IKBKB CEACAM1 } \\
\text { ANXA2 HNRNPA2B1 DNAJB1 HSPD1 EIF3E PSMD1 SNX9 GSK3A GSK3B NPM1 } \\
\text { G3BP2 PARP1 FKBP1A MARK3 RCAN3 HGS DDAH2 SERPING1 TERF1 CAV1 } \\
\text { UBE2I }\end{array}$ \\
\hline GO:0044085 & cellular component biogenesis & $45 / 119$ & $\begin{array}{l}\text { SCFD1 PSMC2 TWF2 KPNB1 STX5 RSL24D1 JUN XPO1 TGFB1 SMAD3 ACE2 BCL2 } \\
\text { CD9 IKBKB ANXA2 DNAJB1 HSPD1 HSPA9 RPL13A EIF3E EIF3I CHMP4B EIF3F } \\
\text { KIF11 SRP54 SNX9 GSK3B NPM1 G3BP1 G3BP2 PARP1 DDX1 FKBP1A PPIH HGS } \\
\text { BAG6 CHMP2B SNAP47 NDUFA10 LAS1L H2AFY2 CHEK2 TERF1 DCTN2 CAV1 }\end{array}$ \\
\hline
\end{tabular}




\section{HCOV-CLUSTER_SET}

\subsection{GO CLASSIFICATION}

Functional Profile of a gene set at specific GO level. Genes are classified according to their GO distribution at a specific level.

\section{MOLECULAR FUNCTION}

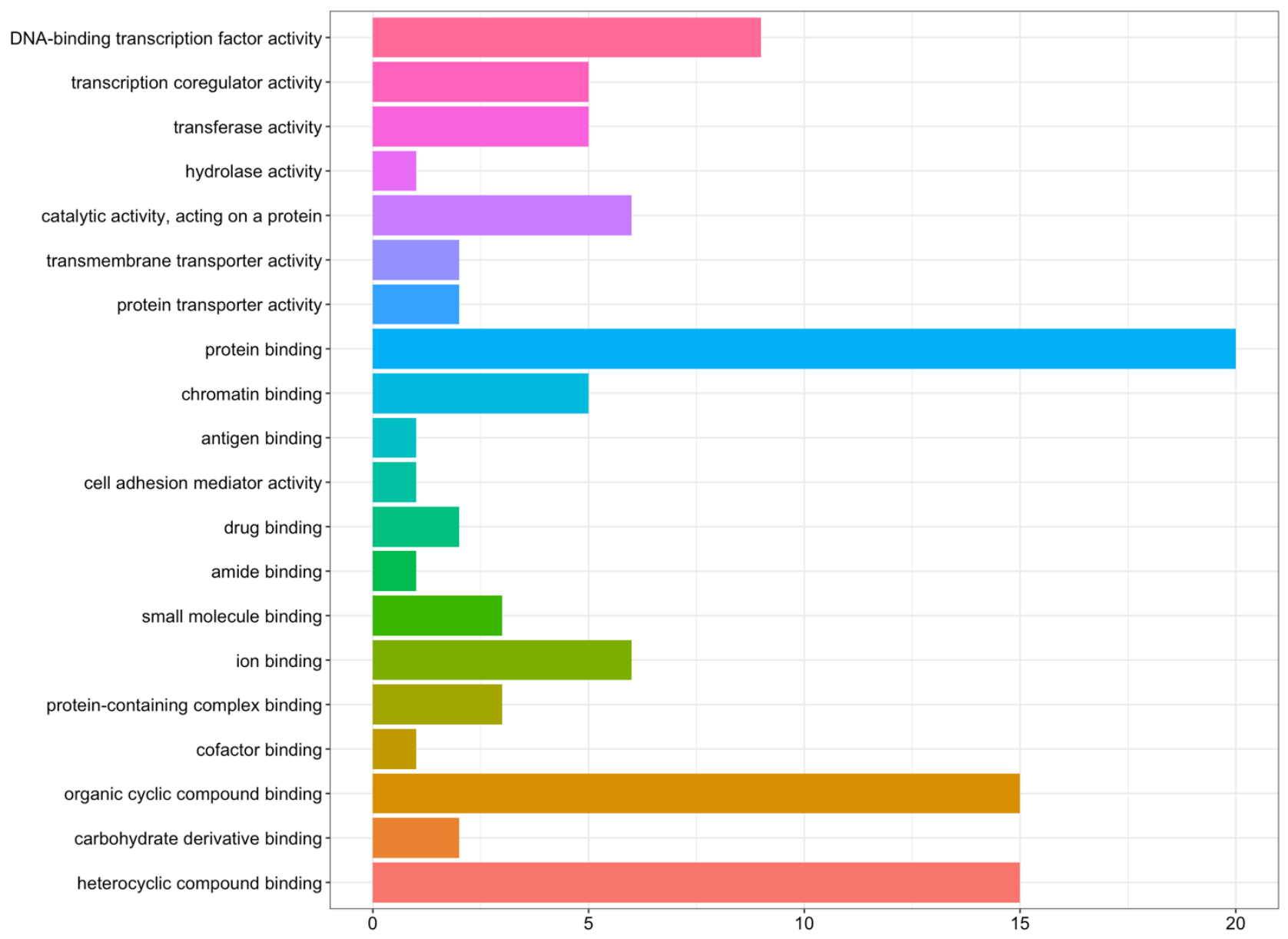




\section{CELLULAR COMPONENT}

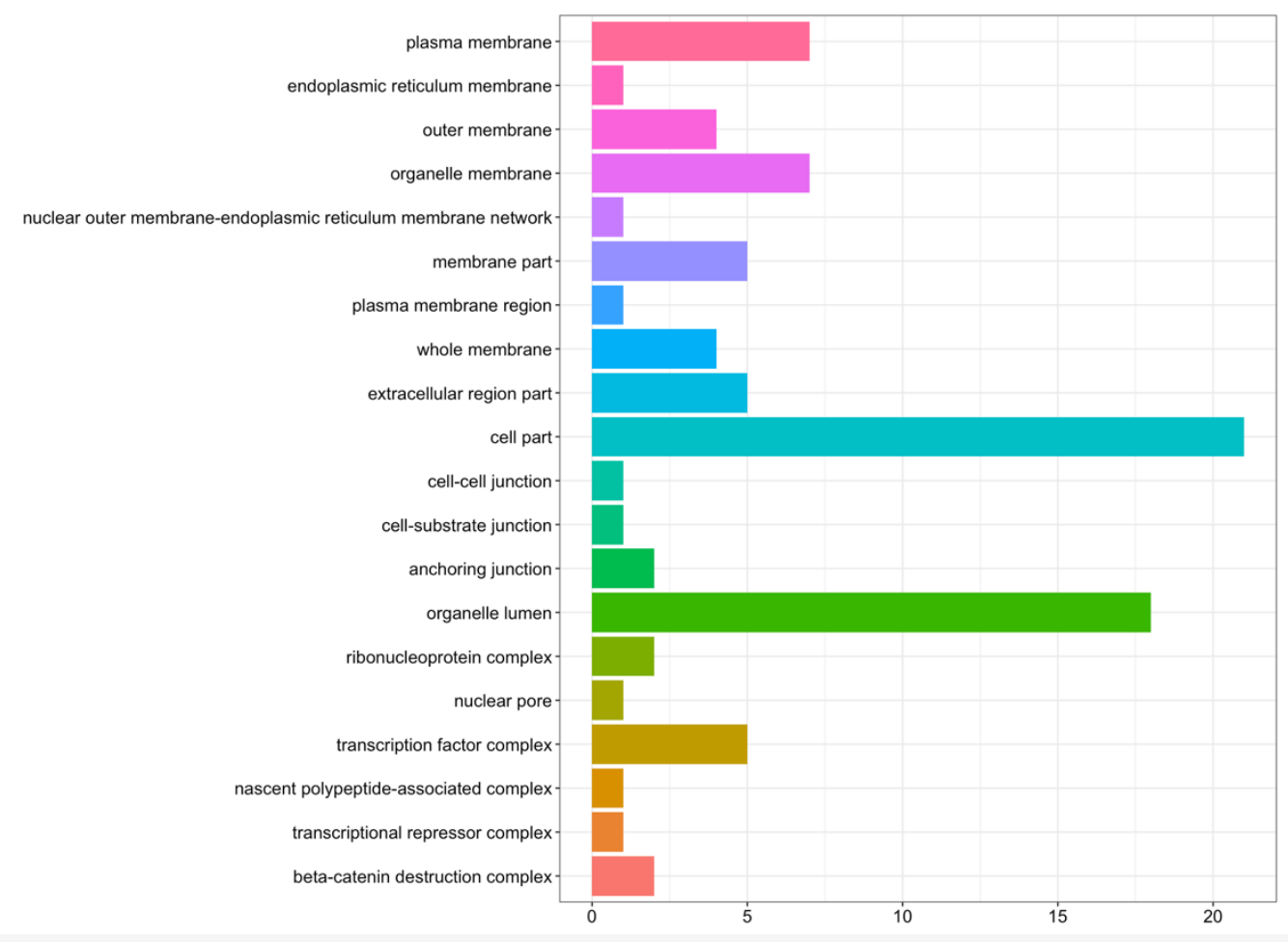

\section{BIOLOGICAL PROCESS}

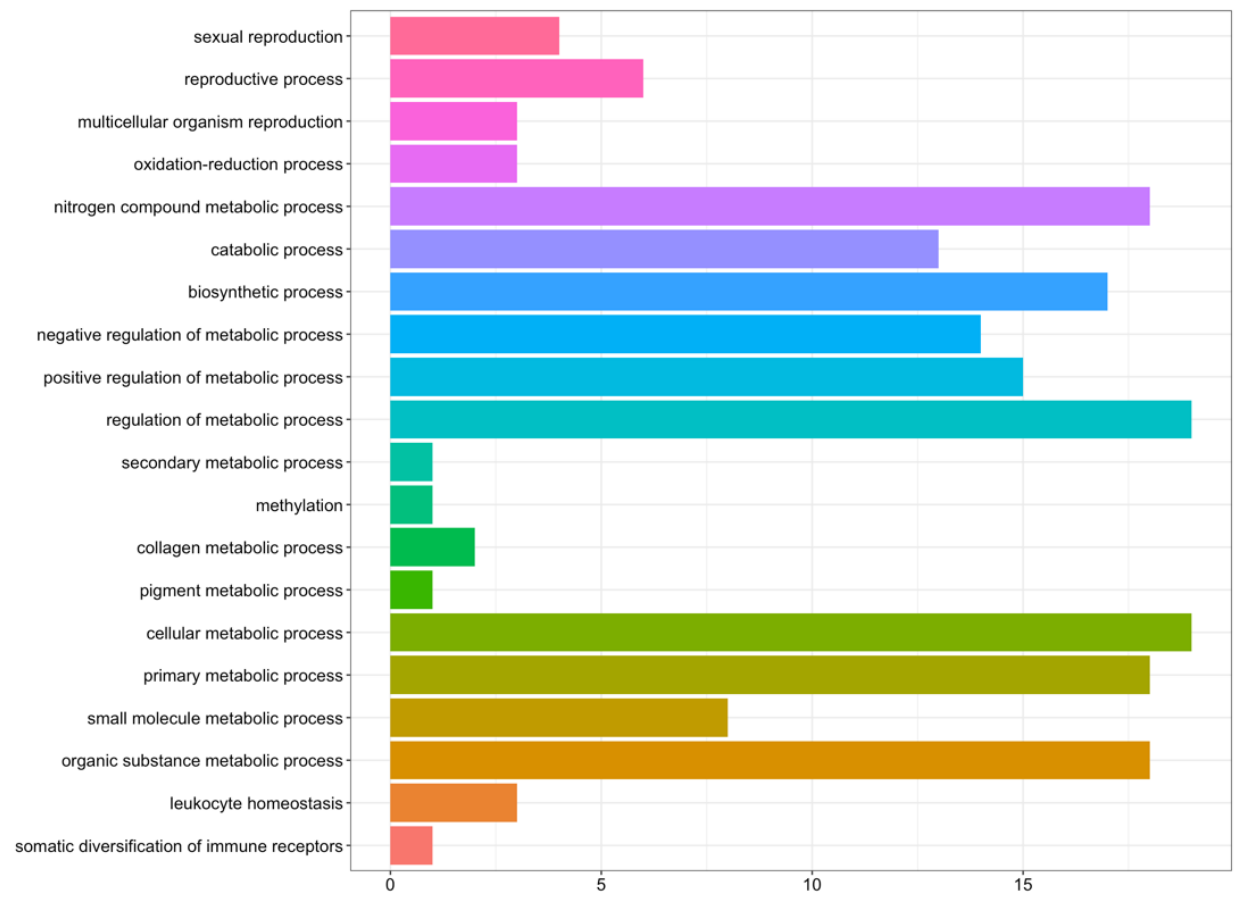




\subsubsection{MOLECULAR FUNCTION}

\begin{tabular}{|c|c|c|c|}
\hline ID & Description & GeneRatio & geneID \\
\hline GO:0003700 & DNA-binding transcription factor activity & $9 / 21$ & TFEB STAT5A PHB JUN STAT3 SMAD3 ATF5 IRF3 PARP1 \\
\hline GO:0003712 & transcription coregulator activity & $5 / 21$ & JUN SMAD3 ATF5 IRF3 NPM1 \\
\hline GO:0016740 & transferase activity & $5 / 21$ & STAT5A SKP2 GSK3A GSK3B PARP1 \\
\hline GO:0016787 & hydrolase activity & $1 / 21$ & PPP1CA \\
\hline GO:0140096 & catalytic activity, acting on a protein & $6 / 21$ & STAT5A PPP1CA SKP2 GSK3A GSK3B PARP1 \\
\hline GO:0022857 & transmembrane transporter activity & $2 / 21$ & MCL1 BCL2 \\
\hline GO:0008565 & protein transporter activity & $2 / 21$ & KPNB1 MCL1 \\
\hline GO:0005515 & protein binding & $20 / 21$ & $\begin{array}{l}\text { TFEB KPNB1 STAT5A PHB JUN STAT3 PPP1CA TGFB1 SMAD3 ATF5 } \\
\text { IRF3 BCL2L1 MCL1 BCL2 SKP2 GSK3A GSK3B NPM1 PARP1 IL6 }\end{array}$ \\
\hline GO:0003682 & chromatin binding & $5 / 21$ & JUN STAT3 SMAD3 ATF5 NPM1 \\
\hline GO:0003823 & antigen binding & $1 / 21$ & TGFB1 \\
\hline GO:0098631 & cell adhesion mediator activity & $1 / 21$ & PPP1CA \\
\hline GO:0008144 & drug binding & $2 / 21$ & GSK3A GSK3B \\
\hline GO:0033218 & amide binding & $1 / 21$ & KPNB1 \\
\hline GO:0036094 & small molecule binding & $3 / 21$ & GSK3A GSK3B PARP1 \\
\hline GO:0043167 & ion binding & $6 / 21$ & KPNB1 PPP1CA SMAD3 GSK3A GSK3B PARP1 \\
\hline GO:0044877 & protein-containing complex binding & $3 / 21$ & PPP1CA SMAD3 NPM1 \\
\hline GO:0048037 & cofactor binding & $1 / 21$ & PARP1 \\
\hline GO:0097159 & organic cyclic compound binding & $15 / 21$ & $\begin{array}{l}\text { TFEB KPNB1 STAT5A PHB JUN STAT3 SMAD3 ATF5 IRF3 BCL2 } \\
\text { NACA GSK3A GSK3B NPM1 PARP1 }\end{array}$ \\
\hline GO:0097367 & carbohydrate derivative binding & $2 / 21$ & GSK3A GSK3B \\
\hline GO:1901363 & heterocyclic compound binding & $15 / 21$ & $\begin{array}{l}\text { TFEB KPNB1 STAT5A PHB JUN STAT3 SMAD3 ATF5 IRF3 BCL2 } \\
\text { NACA GSK3A GSK3B NPM1 PARP1 }\end{array}$ \\
\hline GO:0016247 & channel regulator activity & $1 / 21$ & BCL2 \\
\hline GO:0030234 & enzyme regulator activity & $3 / 21$ & JUN TGFB1 NPM1 \\
\hline GO:0030545 & receptor regulator activity & $2 / 21$ & TGFB1 IL6 \\
\hline GO:0005072 & $\begin{array}{l}\text { transforming growth factor beta receptor, } \\
\text { cytoplasmic mediator activity }\end{array}$ & $1 / 21$ & SMAD3 \\
\hline
\end{tabular}




\subsubsection{CELLULAR COMPONENT}

\begin{tabular}{|c|c|c|c|}
\hline ID & Description & GeneRatio & geneID \\
\hline GO:0005886 & plasma membrane & $7 / 21$ & PHB STAT3 PPP1CA TGFB1 SMAD3 GSK3B IL6 \\
\hline GO:0005789 & endoplasmic reticulum membrane & $1 / 21$ & BCL2 \\
\hline GO:0019867 & outer membrane & $4 / 21$ & PHB BCL2L1 MCL1 BCL2 \\
\hline GO:0031090 & organelle membrane & $7 / 21$ & KPNB1 PHB STAT3 SMAD3 BCL2L1 MCL1 BCL2 \\
\hline GO:0042175 & $\begin{array}{l}\text { nuclear outer membrane-endoplasmic } \\
\text { reticulum membrane network }\end{array}$ & $1 / 21$ & BCL2 \\
\hline GO:0044425 & membrane part & $5 / 21$ & PHB BCL2L1 MCL1 BCL2 IL6 \\
\hline GO:0098590 & plasma membrane region & $1 / 21$ & PHB \\
\hline GO:0098805 & whole membrane & $4 / 21$ & PHB BCL2L1 MCL1 BCL2 \\
\hline GO:0044421 & extracellular region part & $5 / 21$ & KPNB1 PHB PPP1CA TGFB1 IL6 \\
\hline GO:0044464 & cell part & $21 / 21$ & $\begin{array}{l}\text { TFEB KPNB1 STAT5A PHB JUN STAT3 PPP1CA TGFB1 SMAD3 ATF5 } \\
\text { IRF3 BCL2L1 MCL1 BCL2 SKP2 NACA GSK3A GSK3B NPM1 PARP1 IL6 }\end{array}$ \\
\hline GO:0005911 & cell-cell junction & $1 / 21$ & PPP1CA \\
\hline GO:0030055 & cell-substrate junction & $1 / 21$ & NPM1 \\
\hline GO:0070161 & anchoring junction & $2 / 21$ & PPP1CA NPM1 \\
\hline GO:0043233 & organelle lumen & $18 / 21$ & $\begin{array}{l}\text { KPNB1 STAT5A PHB JUN STAT3 PPP1CA TGFB1 SMAD3 ATF5 IRF3 } \\
\text { BCL2L1 MCL1 BCL2 SKP2 GSK3B NPM1 PARP1 IL6 }\end{array}$ \\
\hline GO:1990904 & ribonucleoprotein complex & $2 / 21$ & KPNB1 NPM1 \\
\hline GO:0005643 & nuclear pore & $1 / 21$ & KPNB1 \\
\hline GO:0005667 & transcription factor complex & $5 / 21$ & JUN STAT3 SMAD3 ATF5 PARP1 \\
\hline GO:0005854 & nascent polypeptide-associated complex & $1 / 21$ & NACA \\
\hline GO:0017053 & transcriptional repressor complex & $1 / 21$ & JUN \\
\hline GO:0030877 & beta-catenin destruction complex & $2 / 21$ & GSK3A GSK3B \\
\hline GO:0032993 & protein-DNA complex & $2 / 21$ & NPM1 PARP1 \\
\hline GO:0043235 & receptor complex & $2 / 21$ & SMAD3 IL6 \\
\hline GO:0071141 & SMAD protein complex & $1 / 21$ & SMAD3 \\
\hline GO:0097136 & Bcl-2 family protein complex & $2 / 21$ & BCL2L1 MCL1 \\
\hline GO:0098796 & membrane protein complex & $2 / 21$ & BCL2 IL6 \\
\hline GO:1902494 & catalytic complex & $2 / 21$ & PPP1CA SKP2 \\
\hline GO:1990909 & Wnt signalosome & $1 / 21$ & GSK3B \\
\hline
\end{tabular}




\begin{tabular}{|c|c|c|c|}
\hline ID & Description & GeneRatio & geneID \\
\hline GO:0043227 & membrane-bounded organelle & $21 / 21$ & $\begin{array}{l}\text { TFEB KPNB1 STAT5A PHB JUN STAT3 PPP1CA TGFB1 SMAD3 ATF5 } \\
\text { IRF3 BCL2L1 MCL1 BCL2 SKP2 NACA GSK3A GSK3B NPM1 PARP1 IL6 }\end{array}$ \\
\hline GO:0043228 & non-membrane-bounded organelle & $11 / 21$ & $\begin{array}{l}\text { KPNB1 JUN STAT3 PPP1CA SMAD3 ATF5 BCL2L1 SKP2 GSK3B NPM1 } \\
\text { PARP1 }\end{array}$ \\
\hline GO:0043229 & intracellular organelle & $21 / 21$ & $\begin{array}{l}\text { TFEB KPNB1 STAT5A PHB JUN STAT3 PPP1CA TGFB1 SMAD3 ATF5 } \\
\text { IRF3 BCL2L1 MCL1 BCL2 SKP2 NACA GSK3A GSK3B NPM1 PARP1 IL6 }\end{array}$ \\
\hline GO:0043230 & extracellular organelle & $3 / 21$ & KPNB1 PHB PPP1CA \\
\hline GO:0044422 & organelle part & $18 / 21$ & $\begin{array}{l}\text { KPNB1 STAT5A PHB JUN STAT3 PPP1CA TGFB1 SMAD3 ATF5 IRF3 } \\
\text { BCL2L1 MCL1 BCL2 SKP2 GSK3B NPM1 PARP1 IL6 }\end{array}$ \\
\hline GO:0099572 & postsynaptic specialization & $2 / 21$ & PHB STAT3 \\
\hline GO:0018995 & host & $1 / 21$ & KPNB1 \\
\hline GO:0044217 & other organism part & $1 / 21$ & KPNB1 \\
\hline GO:0044216 & other organism cell & $1 / 21$ & KPNB1 \\
\hline GO:0005615 & extracellular space & $5 / 21$ & KPNB1 PHB PPP1CA TGFB1 IL6 \\
\hline GO:0031012 & extracellular matrix & $1 / 21$ & TGFB1 \\
\hline GO:0072562 & blood microparticle & $1 / 21$ & TGFB1 \\
\hline GO:0044446 & intracellular organelle part & $18 / 21$ & $\begin{array}{l}\text { KPNB1 STAT5A PHB JUN STAT3 PPP1CA TGFB1 SMAD3 ATF5 IRF3 } \\
\text { BCL2L1 MCL1 BCL2 SKP2 GSK3B NPM1 PARP1 IL6 }\end{array}$ \\
\hline GO:0019898 & extrinsic component of membrane & $1 / 21$ & PHB \\
\hline GO:0031224 & intrinsic component of membrane & $5 / 21$ & PHB BCL2L1 MCL1 BCL2 IL6 \\
\hline GO:0044455 & mitochondrial membrane part & $1 / 21$ & PHB \\
\hline GO:0044459 & plasma membrane part & $2 / 21$ & PHB IL6 \\
\hline GO:0008021 & synaptic vesicle & $1 / 21$ & BCL2L1 \\
\hline GO:0030672 & synaptic vesicle membrane & $1 / 21$ & BCL2L1 \\
\hline GO:0048786 & presynaptic active zone & $1 / 21$ & PHB \\
\hline GO:0097060 & synaptic membrane & $1 / 21$ & PHB \\
\hline GO:0098793 & presynapse & $3 / 21$ & PHB PPP1CA BCL2L1 \\
\hline GO:0098794 & postsynapse & $5 / 21$ & PHB STAT3 PPP1CA GSK3A GSK3B \\
\hline GO:0099243 & extrinsic component of synaptic membrane & $1 / 21$ & PHB \\
\hline GO:0005622 & intracellular & $21 / 21$ & $\begin{array}{l}\text { TFEB KPNB1 STAT5A PHB JUN STAT3 PPP1CA TGFB1 SMAD3 ATF5 } \\
\text { IRF3 BCL2L1 MCL1 BCL2 SKP2 NACA GSK3A GSK3B NPM1 PARP1 IL6 }\end{array}$ \\
\hline GO:0008287 & $\begin{array}{l}\text { protein serine/threonine phosphatase } \\
\text { complex }\end{array}$ & $1 / 21$ & PPP1CA \\
\hline GO:0009986 & cell surface & $2 / 21$ & PHB TGFB1 \\
\hline GO:0012505 & endomembrane system & $8 / 21$ & KPNB1 PHB TGFB1 SMAD3 BCL2L1 BCL2 PARP1 IL6 \\
\hline
\end{tabular}




\begin{tabular}{|c|c|c|c|}
\hline ID & Description & GeneRatio & geneID \\
\hline GO:0031975 & envelope & $8 / 21$ & KPNB1 PHB STAT3 SMAD3 BCL2L1 MCL1 BCL2 PARP1 \\
\hline GO:0042995 & cell projection & $4 / 21$ & PPP1CA TGFB1 GSK3A GSK3B \\
\hline GO:0043209 & myelin sheath & $2 / 21$ & PHB BCL2 \\
\hline GO:0044297 & cell body & $3 / 21$ & PPP1CA TGFB1 GSK3A \\
\hline GO:0044424 & intracellular part & $21 / 21$ & $\begin{array}{l}\text { TFEB KPNB1 STAT5A PHB JUN STAT3 PPP1CA TGFB1 SMAD3 ATF5 } \\
\text { IRF3 BCL2L1 MCL1 BCL2 SKP2 NACA GSK3A GSK3B NPM1 PARP1 IL6 }\end{array}$ \\
\hline GO:0044463 & cell projection part & $3 / 21$ & PPP1CA GSK3A GSK3B \\
\hline GO:0071944 & cell periphery & $7 / 21$ & PHB STAT3 PPP1CA TGFB1 SMAD3 GSK3B IL6 \\
\hline GO:0097458 & neuron part & $7 / 21$ & PHB STAT3 PPP1CA TGFB1 BCL2L1 GSK3A GSK3B \\
\hline GO:0044456 & synapse part & $6 / 21$ & PHB STAT3 PPP1CA BCL2L1 GSK3A GSK3B \\
\hline GO:0098685 & Schaffer collateral - CA1 synapse & $1 / 21$ & STAT3 \\
\hline GO:0098978 & glutamatergic synapse & $4 / 21$ & PHB STAT3 PPP1CA GSK3B \\
\hline GO:0098982 & GABA-ergic synapse & $1 / 21$ & PHB \\
\hline GO:0098984 & neuron to neuron synapse & $2 / 21$ & PHB STAT3 \\
\hline
\end{tabular}

\subsubsection{BIOLOGICAL PROCESS}

\begin{tabular}{|c|c|c|c|}
\hline ID & Description & GeneRatio & geneID \\
\hline GO:0019953 & sexual reproduction & $4 / 21$ & STAT3 TGFB1 BCL2L1 BCL2 \\
\hline GO:0022414 & reproductive process & $6 / 21$ & TFEB PHB STAT3 TGFB1 BCL2L1 BCL2 \\
\hline GO:0032504 & multicellular organism reproduction & $3 / 21$ & TGFB1 BCL2L1 BCL2 \\
\hline GO:0055114 & oxidation-reduction process & $3 / 21$ & PPP1CA GSK3A GSK3B \\
\hline GO:0006807 & nitrogen compound metabolic process & $18 / 21$ & $\begin{array}{l}\text { TFEB KPNB1 STAT5A PHB JUN STAT3 PPP1CA TGFB1 SMAD3 ATF5 } \\
\text { IRF3 BCL2 SKP2 GSK3A GSK3B NPM1 PARP1 IL6 }\end{array}$ \\
\hline GO:0009056 & catabolic process & $13 / 21$ & $\begin{array}{l}\text { TFEB KPNB1 PHB STAT3 PPP1CA TGFB1 SMAD3 MCL1 BCL2 SKP2 } \\
\text { GSK3A GSK3B NPM1 }\end{array}$ \\
\hline GO:0009058 & biosynthetic process & $17 / 21$ & $\begin{array}{c}\text { TFEB KPNB1 STAT5A PHB JUN STAT3 PPP1CA TGFB1 SMAD3 ATF5 } \\
\text { IRF3 BCL2 GSK3A GSK3B NPM1 PARP1 IL6 }\end{array}$ \\
\hline GO:0009892 & negative regulation of metabolic process & $14 / 21$ & $\begin{array}{l}\text { PHB JUN STAT3 TGFB1 SMAD3 ATF5 IRF3 MCL1 BCL2 GSK3A GSK3B } \\
\text { NPM1 PARP1 IL6 }\end{array}$ \\
\hline GO:0009893 & positive regulation of metabolic process & $15 / 21$ & $\begin{array}{l}\text { TFEB PHB JUN STAT3 TGFB1 SMAD3 ATF5 IRF3 BCL2 SKP2 GSK3A } \\
\text { GSK3B NPM1 PARP1 IL6 }\end{array}$ \\
\hline GO:0019222 & regulation of metabolic process & $19 / 21$ & $\begin{array}{l}\text { TFEB KPNB1 STAT5A PHB JUN STAT3 PPP1CA TGFB1 SMAD3 ATF5 } \\
\text { IRF3 MCL1 BCL2 SKP2 GSK3A GSK3B NPM1 PARP1 IL6 }\end{array}$ \\
\hline
\end{tabular}




\begin{tabular}{|c|c|c|c|}
\hline ID & Description & GeneRatio & geneID \\
\hline GO:0019748 & secondary metabolic process & $1 / 21$ & BCL2 \\
\hline GO:0032259 & methylation & $1 / 21$ & PARP1 \\
\hline GO:0032963 & collagen metabolic process & $2 / 21$ & TGFB1 IL6 \\
\hline GO:0042440 & pigment metabolic process & $1 / 21$ & BCL2 \\
\hline GO:0044237 & cellular metabolic process & $19 / 21$ & $\begin{array}{l}\text { TFEB KPNB1 STAT5A PHB JUN STAT3 PPP1CA TGFB1 SMAD3 ATF5 } \\
\text { IRF3 MCL1 BCL2 SKP2 GSK3A GSK3B NPM1 PARP1 IL6 }\end{array}$ \\
\hline GO:0044238 & primary metabolic process & $18 / 21$ & $\begin{array}{l}\text { TFEB KPNB1 STAT5A PHB JUN STAT3 PPP1CA TGFB1 SMAD3 ATF5 } \\
\text { IRF3 BCL2 SKP2 GSK3A GSK3B NPM1 PARP1 IL6 }\end{array}$ \\
\hline GO:0044281 & small molecule metabolic process & $8 / 21$ & KPNB1 STAT5A STAT3 PPP1CA TGFB1 GSK3A GSK3B PARP1 \\
\hline GO:0071704 & organic substance metabolic process & $18 / 21$ & $\begin{array}{l}\text { TFEB KPNB1 STAT5A PHB JUN STAT3 PPP1CA TGFB1 SMAD3 ATF5 } \\
\text { IRF3 BCL2 SKP2 GSK3A GSK3B NPM1 PARP1 IL6 }\end{array}$ \\
\hline GO:0001776 & leukocyte homeostasis & $3 / 21$ & TGFB1 BCL2 IL6 \\
\hline GO:0002200 & somatic diversification of immune receptors & $1 / 21$ & TGFB1 \\
\hline GO:0002252 & immune effector process & $8 / 21$ & KPNB1 PHB STAT3 TGFB1 IRF3 BCL2 SKP2 IL6 \\
\hline GO:0002253 & activation of immune response & $3 / 21$ & PHB IRF3 BCL2 \\
\hline GO:0002262 & myeloid cell homeostasis & $2 / 21$ & STAT3 IL6 \\
\hline GO:0002440 & $\begin{array}{l}\text { production of molecular mediator of immune } \\
\text { response }\end{array}$ & $2 / 21$ & TGFB1 IL6 \\
\hline GO:0002507 & tolerance induction & $1 / 21$ & TGFB1 \\
\hline GO:0002520 & immune system development & $7 / 21$ & JUN STAT3 TGFB1 SMAD3 BCL2 PARP1 IL6 \\
\hline GO:0002682 & regulation of immune system process & $8 / 21$ & PHB JUN STAT3 TGFB1 SMAD3 IRF3 BCL2 IL6 \\
\hline GO:0002683 & $\begin{array}{l}\text { negative regulation of immune system } \\
\text { process }\end{array}$ & $1 / 21$ & TGFB1 \\
\hline GO:0002684 & positive regulation of immune system process & $7 / 21$ & PHB JUN STAT3 TGFB1 IRF3 BCL2 IL6 \\
\hline GO:0006955 & immune response & $11 / 21$ & TFEB KPNB1 PHB JUN STAT3 TGFB1 SMAD3 IRF3 BCL2 SKP2 IL6 \\
\hline GO:0045058 & $\mathrm{T}$ cell selection & $3 / 21$ & STAT3 BCL2 IL6 \\
\hline GO:0045321 & leukocyte activation & $7 / 21$ & KPNB1 JUN STAT3 TGFB1 SMAD3 BCL2 IL6 \\
\hline GO:0050900 & leukocyte migration & $2 / 21$ & TGFB1 IL6 \\
\hline GO:0016049 & cell growth & $6 / 21$ & PHB TGFB1 SMAD3 BCL2 GSK3A GSK3B \\
\hline GO:0040008 & regulation of growth & $9 / 21$ & STAT5A PHB STAT3 TGFB1 SMAD3 BCL2L1 BCL2 GSK3A GSK3B \\
\hline GO:0045926 & negative regulation of growth & $5 / 21$ & PHB TGFB1 SMAD3 BCL2 GSK3A \\
\hline GO:0045927 & positive regulation of growth & $1 / 21$ & BCL2 \\
\hline GO:0048589 & developmental growth & $8 / 21$ & STAT5A STAT3 TGFB1 SMAD3 ATF5 BCL2 GSK3A GSK3B \\
\hline
\end{tabular}




\begin{tabular}{|c|c|c|c|}
\hline ID & Description & GeneRatio & geneID \\
\hline GO:0002209 & behavioral defense response & $1 / 21$ & BCL2 \\
\hline GO:0007611 & learning or memory & $1 / 21$ & JUN \\
\hline GO:0007631 & feeding behavior & $1 / 21$ & STAT3 \\
\hline GO:0050795 & regulation of behavior & $1 / 21$ & STAT3 \\
\hline GO:0008284 & positive regulation of cell proliferation & $9 / 21$ & STAT5A JUN STAT3 TGFB1 BCL2L1 BCL2 SKP2 NPM1 IL6 \\
\hline GO:0008285 & negative regulation of cell proliferation & $9 / 21$ & PHB JUN STAT3 TGFB1 SMAD3 ATF5 BCL2 NPM1 IL6 \\
\hline GO:0014009 & glial cell proliferation & $1 / 21$ & IL6 \\
\hline GO:0033002 & muscle cell proliferation & $4 / 21$ & JUN STAT3 SKP2 IL6 \\
\hline GO:0033687 & osteoblast proliferation & $2 / 21$ & SMAD3 BCL2 \\
\hline GO:0042127 & regulation of cell proliferation & $12 / 21$ & $\begin{array}{l}\text { STAT5A PHB JUN STAT3 TGFB1 SMAD3 ATF5 BCL2L1 BCL2 SKP2 } \\
\text { NPM1 IL6 }\end{array}$ \\
\hline GO:0048144 & fibroblast proliferation & $2 / 21$ & JUN TGFB1 \\
\hline GO:0050673 & epithelial cell proliferation & $5 / 21$ & STAT5A JUN STAT3 TGFB1 SMAD3 \\
\hline GO:0061351 & neural precursor cell proliferation & $2 / 21$ & TGFB1 ATF5 \\
\hline GO:0070661 & leukocyte proliferation & $3 / 21$ & TGFB1 BCL2 IL6 \\
\hline GO:0072089 & stem cell proliferation & $1 / 21$ & TGFB1 \\
\hline GO:0007059 & chromosome segregation & $1 / 21$ & KPNB1 \\
\hline GO:0007017 & microtubule-based process & $4 / 21$ & KPNB1 ATF5 GSK3B NPM1 \\
\hline GO:0000075 & cell cycle checkpoint & $2 / 21$ & TGFB1 BCL2L1 \\
\hline GO:0001775 & cell activation & $7 / 21$ & KPNB1 JUN STAT3 TGFB1 SMAD3 BCL2 IL6 \\
\hline GO:0007165 & signal transduction & $18 / 21$ & $\begin{array}{l}\text { KPNB1 STAT5A PHB JUN STAT3 PPP1CA TGFB1 SMAD3 IRF3 BCL2L1 } \\
\text { MCL1 BCL2 SKP2 GSK3A GSK3B NPM1 PARP1 IL6 }\end{array}$ \\
\hline GO:0006928 & movement of cell or subcellular component & $8 / 21$ & KPNB1 STAT5A JUN STAT3 TGFB1 SMAD3 BCL2 IL6 \\
\hline GO:0007049 & cell cycle & $11 / 21$ & $\begin{array}{l}\text { KPNB1 JUN STAT3 PPP1CA TGFB1 SMAD3 ATF5 BCL2L1 BCL2 SKP2 } \\
\text { NPM1 }\end{array}$ \\
\hline GO:0007154 & cell communication & $18 / 21$ & $\begin{array}{l}\text { KPNB1 STAT5A PHB JUN STAT3 PPP1CA TGFB1 SMAD3 IRF3 BCL2L1 } \\
\text { MCL1 BCL2 SKP2 GSK3A GSK3B NPM1 PARP1 IL6 }\end{array}$ \\
\hline GO:0007163 & establishment or maintenance of cell polarity & $1 / 21$ & GSK3B \\
\hline GO:0007272 & ensheathment of neurons & $1 / 21$ & TGFB1 \\
\hline GO:0008219 & cell death & $18 / 21$ & $\begin{array}{l}\text { KPNB1 PHB JUN STAT3 PPP1CA TGFB1 SMAD3 ATF5 IRF3 BCL2L1 } \\
\text { MCL1 BCL2 SKP2 GSK3A GSK3B NPM1 PARP1 IL6 }\end{array}$ \\
\hline GO:0016043 & cellular component organization & $16 / 21$ & $\begin{array}{l}\text { TFEB KPNB1 PHB JUN STAT3 PPP1CA TGFB1 SMAD3 ATF5 BCL2L1 } \\
\text { BCL2 GSK3A GSK3B NPM1 PARP1 IL6 }\end{array}$ \\
\hline GO:0016458 & gene silencing & $3 / 21$ & STAT3 TGFB1 SMAD3 \\
\hline
\end{tabular}




\begin{tabular}{|c|c|c|c|}
\hline ID & Description & GeneRatio & geneID \\
\hline GO:0019725 & cellular homeostasis & $4 / 21$ & TGFB1 SMAD3 MCL1 BCL2 \\
\hline GO:0022402 & cell cycle process & $8 / 21$ & KPNB1 TGFB1 SMAD3 ATF5 BCL2L1 BCL2 SKP2 NPM1 \\
\hline GO:0022412 & $\begin{array}{l}\text { cellular process involved in reproduction in } \\
\text { multicellular organism }\end{array}$ & $3 / 21$ & TGFB1 BCL2L1 BCL2 \\
\hline GO:0030029 & actin filament-based process & $3 / 21$ & TGFB1 SMAD3 BCL2 \\
\hline GO:0032940 & secretion by cell & $5 / 21$ & KPNB1 TGFB1 IRF3 GSK3B IL6 \\
\hline GO:0033059 & cellular pigmentation & $1 / 21$ & BCL2 \\
\hline GO:0044764 & multi-organism cellular process & $1 / 21$ & IRF3 \\
\hline GO:0048522 & positive regulation of cellular process & $19 / 21$ & $\begin{array}{l}\text { TFEB STAT5A PHB JUN STAT3 PPP1CA TGFB1 SMAD3 ATF5 IRF3 } \\
\text { BCL2L1 MCL1 BCL2 SKP2 GSK3A GSK3B NPM1 PARP1 IL6 }\end{array}$ \\
\hline GO:0048523 & negative regulation of cellular process & $15 / 21$ & $\begin{array}{l}\text { PHB JUN STAT3 TGFB1 SMAD3 ATF5 IRF3 BCL2L1 MCL1 BCL2 } \\
\text { GSK3A GSK3B NPM1 PARP1 IL6 }\end{array}$ \\
\hline GO:0048869 & cellular developmental process & $14 / 21$ & $\begin{array}{l}\text { PHB JUN STAT3 TGFB1 SMAD3 ATF5 BCL2L1 MCL1 BCL2 GSK3A } \\
\text { GSK3B NPM1 PARP1 IL6 }\end{array}$ \\
\hline GO:0050794 & regulation of cellular process & $20 / 21$ & $\begin{array}{l}\text { TFEB KPNB1 STAT5A PHB JUN STAT3 PPP1CA TGFB1 SMAD3 ATF5 } \\
\text { IRF3 BCL2L1 MCL1 BCL2 SKP2 GSK3A GSK3B NPM1 PARP1 IL6 }\end{array}$ \\
\hline GO:0051301 & cell division & $3 / 21$ & PPP1CA TGFB1 BCL2L1 \\
\hline GO:0051716 & cellular response to stimulus & $18 / 21$ & $\begin{array}{l}\text { KPNB1 STAT5A PHB JUN STAT3 PPP1CA TGFB1 SMAD3 IRF3 BCL2L1 } \\
\text { MCL1 BCL2 SKP2 GSK3A GSK3B NPM1 PARP1 IL6 }\end{array}$ \\
\hline GO:0061919 & process utilizing autophagic mechanism & $4 / 21$ & TFEB MCL1 BCL2 GSK3A \\
\hline GO:0097194 & execution phase of apoptosis & $2 / 21$ & KPNB1 BCL2L1 \\
\hline GO:0006808 & regulation of nitrogen utilization & $1 / 21$ & BCL2 \\
\hline GO:0003006 & $\begin{array}{l}\text { developmental process involved in } \\
\text { reproduction }\end{array}$ & $5 / 21$ & TFEB PHB TGFB1 BCL2L1 BCL2 \\
\hline GO:0009566 & fertilization & $1 / 21$ & BCL2L1 \\
\hline GO:0044703 & multi-organism reproductive process & $4 / 21$ & STAT3 TGFB1 BCL2L1 BCL2 \\
\hline GO:0048609 & multicellular organismal reproductive process & $3 / 21$ & TGFB1 BCL2L1 BCL2 \\
\hline GO:0007155 & cell adhesion & $6 / 21$ & PPP1CA TGFB1 SMAD3 BCL2 GSK3B IL6 \\
\hline GO:0007267 & cell-cell signaling & $6 / 21$ & STAT3 PPP1CA SMAD3 GSK3A GSK3B IL6 \\
\hline GO:0023051 & regulation of signaling & $15 / 21$ & $\begin{array}{c}\text { PHB JUN STAT3 PPP1CA TGFB1 SMAD3 IRF3 BCL2L1 MCL1 BCL2 } \\
\text { SKP2 GSK3A GSK3B PARP1 IL6 }\end{array}$ \\
\hline GO:0023056 & positive regulation of signaling & $15 / 21$ & $\begin{array}{c}\text { PHB JUN STAT3 PPP1CA TGFB1 SMAD3 IRF3 BCL2L1 MCL1 BCL2 } \\
\text { SKP2 GSK3A GSK3B PARP1 IL6 }\end{array}$ \\
\hline GO:0023057 & negative regulation of signaling & $9 / 21$ & PHB TGFB1 SMAD3 BCL2L1 MCL1 BCL2 GSK3A GSK3B IL6 \\
\hline GO:0001503 & ossification & $5 / 21$ & PHB TGFB1 SMAD3 BCL2 IL6 \\
\hline GO:0001763 & morphogenesis of a branching structure & $3 / 21$ & PPP1CA TGFB1 BCL2 \\
\hline
\end{tabular}




\begin{tabular}{|c|c|c|c|}
\hline ID & Description & GeneRatio & geneID \\
\hline GO:0001816 & cytokine production & $4 / 21$ & TGFB1 SMAD3 IRF3 IL6 \\
\hline GO:0003008 & system process & $7 / 21$ & JUN TGFB1 SMAD3 BCL2 GSK3A GSK3B PARP1 \\
\hline GO:0007275 & multicellular organism development & $16 / 21$ & $\begin{array}{l}\text { TFEB STAT5A PHB JUN STAT3 PPP1CA TGFB1 SMAD3 ATF5 BCL2L1 } \\
\text { MCL1 BCL2 GSK3A GSK3B PARP1 IL6 }\end{array}$ \\
\hline GO:0007389 & pattern specification process & $1 / 21$ & SMAD3 \\
\hline GO:0009791 & post-embryonic development & $2 / 21$ & ATF5 BCL2 \\
\hline GO:0019827 & stem cell population maintenance & $1 / 21$ & STAT3 \\
\hline GO:0022404 & molting cycle process & $1 / 21$ & BCL2 \\
\hline GO:0032922 & circadian regulation of gene expression & $1 / 21$ & PPP1CA \\
\hline GO:0033555 & multicellular organismal response to stress & $1 / 21$ & BCL2 \\
\hline GO:0035264 & multicellular organism growth & $4 / 21$ & STAT5A STAT3 ATF5 BCL2 \\
\hline GO:0035265 & organ growth & $2 / 21$ & BCL2 GSK3A \\
\hline GO:0042303 & molting cycle & $1 / 21$ & BCL2 \\
\hline GO:0044706 & multi-multicellular organism process & $2 / 21$ & TGFB1 BCL2 \\
\hline GO:0048771 & tissue remodeling & $2 / 21$ & TGFB1 IL6 \\
\hline GO:0048871 & multicellular organismal homeostasis & $3 / 21$ & STAT3 BCL2 IL6 \\
\hline GO:0050817 & coagulation & $1 / 21$ & IL6 \\
\hline GO:0051239 & regulation of multicellular organismal process & $11 / 21$ & $\begin{array}{l}\text { STAT5A JUN STAT3 TGFB1 SMAD3 IRF3 BCL2 GSK3A GSK3B PARP1 } \\
\text { IL6 }\end{array}$ \\
\hline GO:0051240 & $\begin{array}{l}\text { positive regulation of multicellular } \\
\text { organismal process }\end{array}$ & $10 / 21$ & STAT5A JUN STAT3 TGFB1 SMAD3 IRF3 BCL2 GSK3A PARP1 IL6 \\
\hline GO:0051241 & $\begin{array}{l}\text { negative regulation of multicellular } \\
\text { organismal process }\end{array}$ & $8 / 21$ & STAT3 TGFB1 SMAD3 IRF3 BCL2 GSK3A GSK3B IL6 \\
\hline GO:0090130 & tissue migration & $3 / 21$ & STAT5A JUN TGFB1 \\
\hline GO:0009653 & anatomical structure morphogenesis & $10 / 21$ & JUN STAT3 PPP1CA TGFB1 SMAD3 BCL2L1 BCL2 GSK3A GSK3B IL6 \\
\hline GO:0007568 & aging & $5 / 21$ & JUN STAT3 TGFB1 BCL2 NPM1 \\
\hline GO:0048646 & $\begin{array}{l}\text { anatomical structure formation involved in } \\
\text { morphogenesis }\end{array}$ & $5 / 21$ & JUN STAT3 TGFB1 SMAD3 IL6 \\
\hline GO:0021700 & developmental maturation & $1 / 21$ & BCL2 \\
\hline GO:0048856 & anatomical structure development & $16 / 21$ & $\begin{array}{l}\text { TFEB STAT5A PHB JUN STAT3 PPP1CA TGFB1 SMAD3 ATF5 BCL2L1 } \\
\text { MCL1 BCL2 GSK3A GSK3B PARP1 IL6 }\end{array}$ \\
\hline GO:0050793 & regulation of developmental process & $10 / 21$ & STAT5A JUN STAT3 TGFB1 SMAD3 BCL2 GSK3A GSK3B PARP1 IL6 \\
\hline GO:0051093 & negative regulation of developmental process & $7 / 21$ & STAT3 TGFB1 SMAD3 BCL2 GSK3A GSK3B IL6 \\
\hline GO:0051094 & positive regulation of developmental process & $7 / 21$ & JUN STAT3 TGFB1 SMAD3 BCL2 PARP1 IL6 \\
\hline
\end{tabular}




\begin{tabular}{|c|c|c|c|}
\hline ID & Description & GeneRatio & geneID \\
\hline GO:0098727 & maintenance of cell number & $1 / 21$ & STAT3 \\
\hline GO:0040012 & regulation of locomotion & $7 / 21$ & STAT5A JUN STAT3 TGFB1 SMAD3 BCL2 IL6 \\
\hline GO:0040013 & negative regulation of locomotion & $3 / 21$ & STAT3 TGFB1 BCL2 \\
\hline GO:0040017 & positive regulation of locomotion & $7 / 21$ & STAT5A JUN STAT3 TGFB1 SMAD3 BCL2 IL6 \\
\hline GO:0042330 & taxis & $3 / 21$ & TGFB1 SMAD3 IL6 \\
\hline GO:0048870 & cell motility & $7 / 21$ & STAT5A JUN STAT3 TGFB1 SMAD3 BCL2 IL6 \\
\hline GO:0007623 & circadian rhythm & $4 / 21$ & JUN PPP1CA ATF5 GSK3B \\
\hline GO:0043902 & positive regulation of multi-organism process & $1 / 21$ & JUN \\
\hline GO:0044089 & $\begin{array}{l}\text { positive regulation of cellular component } \\
\text { biogenesis }\end{array}$ & $5 / 21$ & JUN TGFB1 SMAD3 GSK3B PARP1 \\
\hline GO:0045785 & positive regulation of cell adhesion & $4 / 21$ & TGFB1 SMAD3 GSK3B IL6 \\
\hline GO:0048087 & $\begin{array}{l}\text { positive regulation of developmental } \\
\text { pigmentation }\end{array}$ & $1 / 21$ & BCL2 \\
\hline GO:0048584 & positive regulation of response to stimulus & $15 / 21$ & $\begin{array}{c}\text { PHB JUN STAT3 PPP1CA TGFB1 SMAD3 IRF3 BCL2L1 MCL1 BCL2 } \\
\text { SKP2 GSK3A GSK3B PARP1 IL6 }\end{array}$ \\
\hline GO:0051091 & $\begin{array}{l}\text { positive regulation of DNA-binding } \\
\text { transcription factor activity }\end{array}$ & $4 / 21$ & TGFB1 SMAD3 NPM1 IL6 \\
\hline GO:1903829 & $\begin{array}{l}\text { positive regulation of cellular protein } \\
\text { localization }\end{array}$ & $6 / 21$ & TGFB1 SMAD3 BCL2 GSK3A GSK3B PARP1 \\
\hline GO: 1904181 & $\begin{array}{l}\text { positive regulation of membrane } \\
\text { depolarization }\end{array}$ & $1 / 21$ & PARP1 \\
\hline GO:1904951 & $\begin{array}{l}\text { positive regulation of establishment of protein } \\
\text { localization }\end{array}$ & $7 / 21$ & TGFB1 SMAD3 IRF3 BCL2 GSK3A GSK3B IL6 \\
\hline GO:0043901 & negative regulation of multi-organism process & $1 / 21$ & JUN \\
\hline GO:0048585 & negative regulation of response to stimulus & $9 / 21$ & PHB TGFB1 SMAD3 BCL2L1 MCL1 BCL2 GSK3A GSK3B IL6 \\
\hline GO:0051051 & negative regulation of transport & $3 / 21$ & TGFB1 BCL2 GSK3A \\
\hline GO: 1903828 & $\begin{array}{l}\text { negative regulation of cellular protein } \\
\text { localization }\end{array}$ & $3 / 21$ & TGFB1 BCL2L1 GSK3B \\
\hline GO: 1904180 & $\begin{array}{l}\text { negative regulation of membrane } \\
\text { depolarization }\end{array}$ & $1 / 21$ & BCL2 \\
\hline GO: 1905953 & negative regulation of lipid localization & $1 / 21$ & IL6 \\
\hline GO:0030155 & regulation of cell adhesion & $5 / 21$ & TGFB1 SMAD3 BCL2 GSK3B IL6 \\
\hline GO:0032879 & regulation of localization & $12 / 21$ & $\begin{array}{l}\text { STAT5A JUN STAT3 TGFB1 SMAD3 IRF3 BCL2L1 BCL2 GSK3A GSK3B } \\
\text { PARP1 IL6 }\end{array}$ \\
\hline
\end{tabular}




\begin{tabular}{|c|c|c|c|}
\hline ID & Description & GeneRatio & geneID \\
\hline GO:0043900 & regulation of multi-organism process & $2 / 21$ & JUN BCL2 \\
\hline GO:0044087 & regulation of cellular component biogenesis & $6 / 21$ & JUN TGFB1 SMAD3 GSK3B NPM1 PARP1 \\
\hline GO:0048070 & regulation of developmental pigmentation & $1 / 21$ & BCL2 \\
\hline GO:0048518 & positive regulation of biological process & $19 / 21$ & $\begin{array}{l}\text { TFEB STAT5A PHB JUN STAT3 PPP1CA TGFB1 SMAD3 ATF5 IRF3 } \\
\text { BCL2L1 MCL1 BCL2 SKP2 GSK3A GSK3B NPM1 PARP1 IL6 }\end{array}$ \\
\hline GO:0048519 & negative regulation of biological process & $15 / 21$ & $\begin{array}{c}\text { PHB JUN STAT3 TGFB1 SMAD3 ATF5 IRF3 BCL2L1 MCL1 BCL2 } \\
\text { GSK3A GSK3B NPM1 PARP1 IL6 }\end{array}$ \\
\hline GO:0048583 & regulation of response to stimulus & $16 / 21$ & $\begin{array}{l}\text { PHB JUN STAT3 PPP1CA TGFB1 SMAD3 IRF3 BCL2L1 MCL1 BCL2 } \\
\text { SKP2 GSK3A GSK3B NPM1 PARP1 IL6 }\end{array}$ \\
\hline GO:0006950 & response to stress & $16 / 21$ & $\begin{array}{l}\text { PHB JUN STAT3 PPP1CA TGFB1 SMAD3 IRF3 BCL2L1 MCL1 BCL2 } \\
\text { SKP2 GSK3A GSK3B NPM1 PARP1 IL6 }\end{array}$ \\
\hline GO:0009605 & response to external stimulus & $10 / 21$ & PHB JUN PPP1CA TGFB1 SMAD3 IRF3 BCL2L1 BCL2 SKP2 IL6 \\
\hline GO:0009607 & response to biotic stimulus & $9 / 21$ & JUN TGFB1 SMAD3 IRF3 BCL2L1 BCL2 SKP2 GSK3B IL6 \\
\hline GO:0009628 & response to abiotic stimulus & $9 / 21$ & JUN PPP1CA TGFB1 SMAD3 BCL2L1 BCL2 GSK3B NPM1 PARP1 \\
\hline GO:0009719 & response to endogenous stimulus & $13 / 21$ & $\begin{array}{l}\text { STAT5A PHB JUN STAT3 TGFB1 SMAD3 BCL2L1 BCL2 SKP2 GSK3A } \\
\text { GSK3B PARP1 IL6 }\end{array}$ \\
\hline GO:0042221 & response to chemical & $17 / 21$ & $\begin{array}{l}\text { STAT5A PHB JUN STAT3 PPP1CA TGFB1 SMAD3 IRF3 BCL2L1 MCL1 } \\
\text { BCL2 SKP2 GSK3A GSK3B NPM1 PARP1 IL6 }\end{array}$ \\
\hline GO:0043500 & muscle adaptation & $3 / 21$ & SMAD3 GSK3A PARP1 \\
\hline GO:0051606 & detection of stimulus & $1 / 21$ & PARP1 \\
\hline GO:0072376 & protein activation cascade & $1 / 21$ & PHB \\
\hline GO:0031503 & protein-containing complex localization & $1 / 21$ & NPM1 \\
\hline GO:0033036 & macromolecule localization & $13 / 21$ & $\begin{array}{l}\text { KPNB1 STAT3 TGFB1 SMAD3 IRF3 BCL2L1 MCL1 BCL2 GSK3A } \\
\text { GSK3B NPM1 PARP1 IL6 }\end{array}$ \\
\hline GO:0051234 & establishment of localization & $14 / 21$ & $\begin{array}{l}\text { KPNB1 STAT5A PHB STAT3 TGFB1 SMAD3 IRF3 BCL2L1 MCL1 BCL2 } \\
\text { GSK3A GSK3B NPM1 IL6 }\end{array}$ \\
\hline GO:0051235 & maintenance of location & $2 / 21$ & TGFB1 IL6 \\
\hline GO:0051641 & cellular localization & $10 / 21$ & $\begin{array}{l}\text { KPNB1 STAT3 TGFB1 SMAD3 BCL2L1 BCL2 GSK3A GSK3B NPM1 } \\
\text { PARP1 }\end{array}$ \\
\hline GO:0051674 & localization of cell & $7 / 21$ & STAT5A JUN STAT3 TGFB1 SMAD3 BCL2 IL6 \\
\hline GO: 1902579 & multi-organism localization & $1 / 21$ & KPNB1 \\
\hline GO:0051707 & response to other organism & $8 / 21$ & JUN TGFB1 SMAD3 IRF3 BCL2L1 BCL2 SKP2 IL6 \\
\hline GO:0044419 & interspecies interaction between organisms & $9 / 21$ & KPNB1 JUN STAT3 TGFB1 SMAD3 IRF3 BCL2L1 BCL2 NPM1 \\
\hline GO:0050789 & regulation of biological process & $20 / 21$ & $\begin{array}{l}\text { TFEB KPNB1 STAT5A PHB JUN STAT3 PPP1CA TGFB1 SMAD3 ATF5 } \\
\text { IRF3 BCL2L1 MCL1 BCL2 SKP2 GSK3A GSK3B NPM1 PARP1 IL6 }\end{array}$ \\
\hline GO:0065008 & regulation of biological quality & $15 / 21$ & $\begin{array}{l}\text { STAT5A PHB JUN STAT3 PPP1CA TGFB1 SMAD3 BCL2L1 MCL1 BCL2 } \\
\text { GSK3A GSK3B NPM1 PARP1 IL6 }\end{array}$ \\
\hline
\end{tabular}




\begin{tabular}{|c|c|c|c|}
\hline ID & Description & GeneRatio & geneID \\
\hline GO:0065009 & regulation of molecular function & $11 / 21$ & $\begin{array}{l}\text { JUN STAT3 PPP1CA TGFB1 SMAD3 BCL2 GSK3A GSK3B NPM1 PARP1 } \\
\text { IL6 }\end{array}$ \\
\hline GO:0044085 & cellular component biogenesis & $8 / 21$ & KPNB1 JUN TGFB1 SMAD3 BCL2 GSK3B NPM1 PARP1 \\
\hline
\end{tabular}


GO ENRICHMENT: 


\section{HCOV-HOST_SET}

\subsection{GO ENRICHMENT}

GO Enrichment Analysis after FDR control of a gene set.

\section{MOLECULAR FUNCTION}

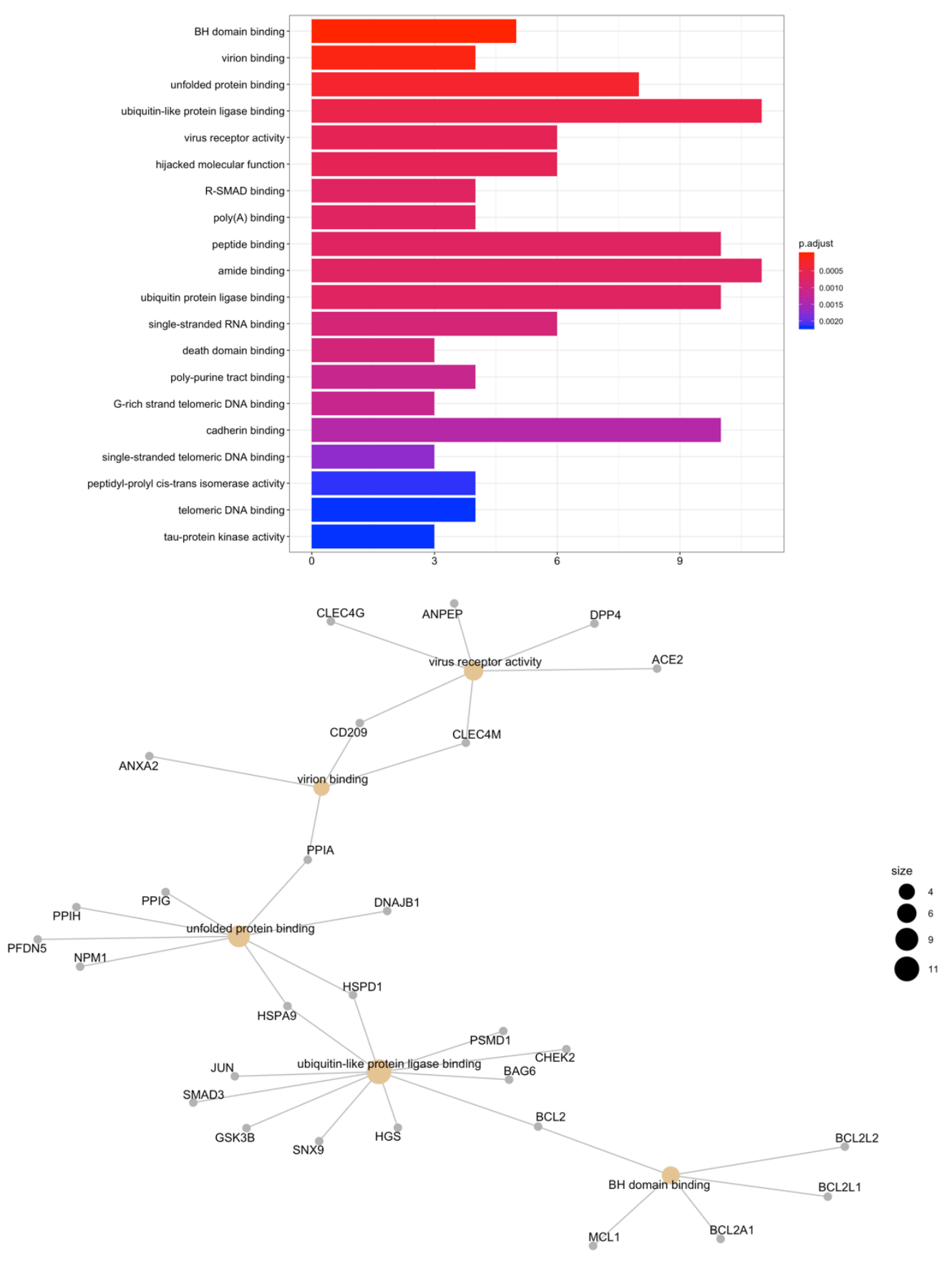




\section{CELLULAR COMPONENT}

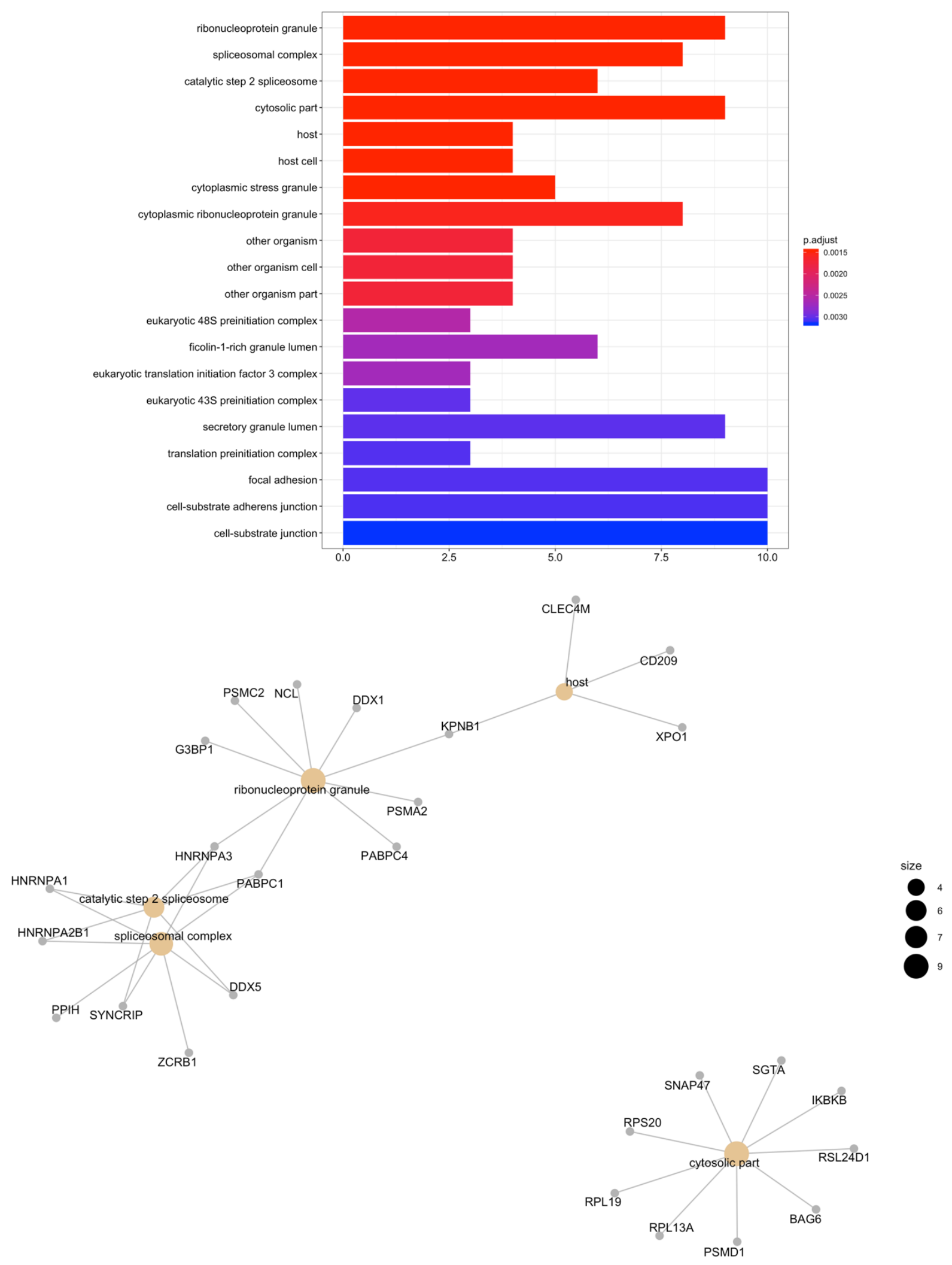




\section{BIOLOGICAL PROCESS}

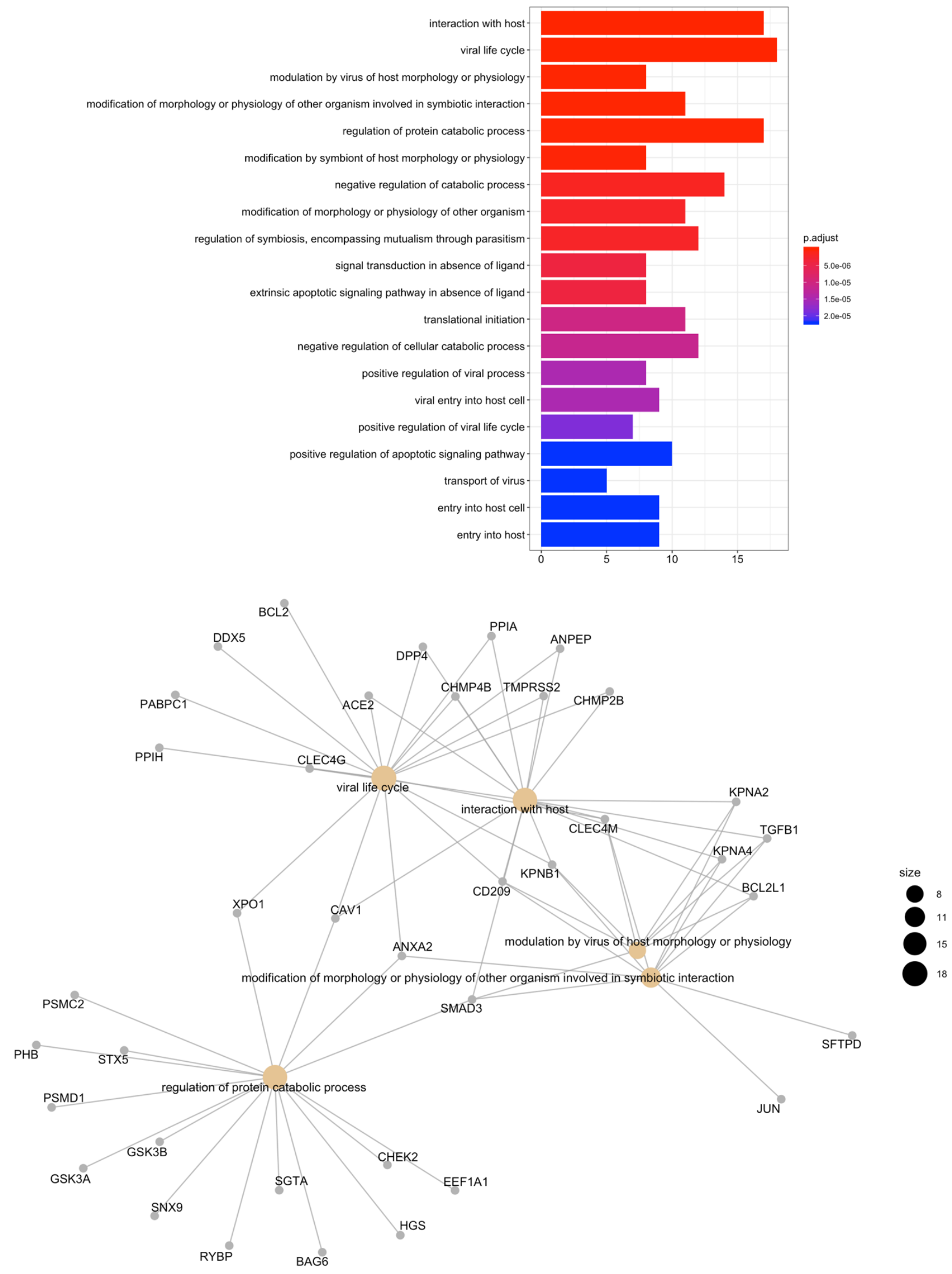




\subsubsection{MOLECULAR FUNCTION}

\begin{tabular}{|c|c|c|c|c|c|}
\hline ID & Description & GeneRatio & BgRatio & p.adjust & geneID \\
\hline GO:0051400 & $\mathrm{BH}$ domain binding & $5 / 117$ & $10 / 17632$ & 0 & BCL2L1 BCL2L2 MCL1 BCL2A1 BCL2 \\
\hline GO:0046790 & virion binding & $4 / 117$ & $10 / 17632$ & 0 & CD209 CLEC4M PPIA ANXA2 \\
\hline GO:0051082 & unfolded protein binding & $8 / 117$ & $127 / 17632$ & 0 & $\begin{array}{l}\text { PPIA DNAJB1 HSPD1 HSPA9 NPM1 PPIG } \\
\text { PPIH PFDN5 }\end{array}$ \\
\hline GO:0044389 & $\begin{array}{c}\text { ubiquitin-like protein ligase } \\
\text { binding }\end{array}$ & $11 / 117$ & $301 / 17632$ & 0 & $\begin{array}{l}\text { JUN SMAD3 BCL2 HSPD1 HSPA9 } \\
\text { PSMD1 SNX9 GSK3B HGS BAG6 CHEK2 }\end{array}$ \\
\hline GO:0001618 & virus receptor activity & $6 / 117$ & $74 / 17632$ & 0.001 & $\begin{array}{c}\text { ACE2 CLEC4G CD209 CLEC4M ANPEP } \\
\text { DPP4 }\end{array}$ \\
\hline GO:0104005 & hijacked molecular function & $6 / 117$ & $74 / 17632$ & 0.001 & $\begin{array}{c}\text { ACE2 CLEC4G CD209 CLEC4M ANPEP } \\
\text { DPP4 }\end{array}$ \\
\hline GO:0070412 & R-SMAD binding & $4 / 117$ & $23 / 17632$ & 0.001 & JUN SMAD3 PARP1 DDX5 \\
\hline GO:0008143 & poly(A) binding & $4 / 117$ & $24 / 17632$ & 0.001 & SYNCRIP PABPC1 PABPC4 DDX1 \\
\hline GO:0042277 & peptide binding & $10 / 117$ & $285 / 17632$ & 0.001 & $\begin{array}{l}\text { KPNB1 CD209 CLEC4M KPNA2 PPIA } \\
\text { ANPEP HSPD1 SRP54 PPIG PPIH }\end{array}$ \\
\hline GO:0033218 & amide binding & $11 / 117$ & $348 / 17632$ & 0.001 & $\begin{array}{l}\text { KPNB1 ACBD5 CD209 CLEC4M KPNA2 } \\
\text { PPIA ANPEP HSPD1 SRP54 PPIG PPIH }\end{array}$ \\
\hline GO:0031625 & ubiquitin protein ligase binding & $10 / 117$ & $286 / 17632$ & 0.001 & $\begin{array}{l}\text { JUN SMAD3 BCL2 HSPD1 HSPA9 } \\
\text { PSMD1 SNX9 GSK3B BAG6 CHEK2 }\end{array}$ \\
\hline GO:0003727 & single-stranded RNA binding & $6 / 117$ & $90 / 17632$ & 0.001 & $\begin{array}{l}\text { HNRNPA1 SYNCRIP PTBP1 PABPC1 } \\
\text { PABPC4 DDX1 }\end{array}$ \\
\hline GO:0070513 & death domain binding & $3 / 117$ & $10 / 17632$ & 0.001 & BCL2L1 MCL1 BCL2 \\
\hline GO:0070717 & poly-purine tract binding & $4 / 117$ & $30 / 17632$ & 0.001 & SYNCRIP PABPC1 PABPC4 DDX1 \\
\hline GO:0098505 & $\begin{array}{c}\text { G-rich strand telomeric DNA } \\
\text { binding }\end{array}$ & $3 / 117$ & $11 / 17632$ & 0.001 & HNRNPA1 HNRNPA2B1 TERF1 \\
\hline GO:0045296 & cadherin binding & $10 / 117$ & $323 / 17632$ & 0.001 & $\begin{array}{l}\text { TWF2 YKT6 STX5 PPP1CA ANXA2 } \\
\text { DNAJB1 EIF3E CHMP4B SNX9 CHMP2B }\end{array}$ \\
\hline GO:0043047 & $\begin{array}{l}\text { single-stranded telomeric DNA } \\
\text { binding }\end{array}$ & $3 / 117$ & $13 / 17632$ & 0.002 & HNRNPA1 HNRNPA2B1 TERF1 \\
\hline GO:0003755 & $\begin{array}{l}\text { peptidyl-prolyl cis-trans isomerase } \\
\text { activity }\end{array}$ & $4 / 117$ & $37 / 17632$ & 0.002 & PPIA FKBP1A PPIG PPIH \\
\hline GO:0042162 & telomeric DNA binding & $4 / 117$ & $38 / 17632$ & 0.002 & HNRNPA1 HNRNPA2B1 NCL TERF1 \\
\hline GO:0050321 & tau-protein kinase activity & $3 / 117$ & $15 / 17632$ & 0.002 & GSK3A GSK3B MARK3 \\
\hline GO:0098847 & $\begin{array}{l}\text { sequence-specific single stranded } \\
\text { DNA binding }\end{array}$ & $3 / 117$ & $15 / 17632$ & 0.002 & HNRNPA1 HNRNPA2B1 TERF1 \\
\hline
\end{tabular}




\begin{tabular}{|c|c|c|c|c|c|}
\hline ID & Description & GeneRatio & BgRatio & p.adjust & geneID \\
\hline GO:0016859 & cis-trans isomerase activity & $4 / 117$ & $41 / 17632$ & 0.003 & PPIA FKBP1A PPIG PPIH \\
\hline GO:0002020 & protease binding & $6 / 117$ & $125 / 17632$ & 0.003 & $\begin{array}{c}\text { BCL2 DPP4 ANXA2 HSPD1 GSK3B } \\
\text { BAG6 }\end{array}$ \\
\hline GO:0046332 & SMAD binding & $5 / 117$ & $80 / 17632$ & 0.003 & JUN SMAD3 PARP1 FKBP1A DDX5 \\
\hline GO:0043021 & ribonucleoprotein complex binding & $6 / 117$ & $128 / 17632$ & 0.003 & PPP1CA SRP54 NPM1 PPIH BAG6 DDX5 \\
\hline GO:0016018 & cyclosporin A binding & $3 / 117$ & $19 / 17632$ & 0.004 & PPIA PPIG PPIH \\
\hline GO:0140142 & nucleocytoplasmic carrier activity & $3 / 117$ & $19 / 17632$ & 0.004 & XPO1 KPNA2 KPNA4 \\
\hline GO:0008565 & protein transporter activity & $5 / 117$ & $88 / 17632$ & 0.004 & KPNB1 XPO1 KPNA2 MCL1 KPNA4 \\
\hline GO:0050839 & cell adhesion molecule binding & $11 / 117$ & $486 / 17632$ & 0.005 & $\begin{array}{l}\text { TWF2 YKT6 STX5 PPP1CA CD9 ANXA2 } \\
\text { DNAJB1 EIF3E CHMP4B SNX9 CHMP2B }\end{array}$ \\
\hline GO:0047485 & protein $\mathrm{N}$-terminus binding & $5 / 117$ & $107 / 17632$ & 0.009 & SCFD1 STX5 TGFB1 EIF3E PARP1 \\
\hline GO:0036002 & pre-mRNA binding & $4 / 117$ & $62 / 17632$ & 0.009 & HNRNPA1 PTBP1 HNRNPA2B1 DDX5 \\
\hline
\end{tabular}

\subsubsection{CELLULAR COMPONENT}

\begin{tabular}{|c|c|c|c|c|c|}
\hline ID & Description & GeneRatio & BgRatio & p.adjust & geneID \\
\hline GO:0035770 & ribonucleoprotein granule & $9 / 119$ & $219 / 19659$ & 0.001 & $\begin{array}{l}\text { PSMC2 KPNB1 HNRNPA3 PABPC1 } \\
\text { PABPC4 G3BP1 NCL DDX1 PSMA2 }\end{array}$ \\
\hline GO:0005681 & spliceosomal complex & $8 / 119$ & $179 / 19659$ & 0.001 & $\begin{array}{c}\text { HNRNPA1 ZCRB1 SYNCRIP } \\
\text { HNRNPA2B1 HNRNPA3 PABPC1 PPIH } \\
\text { DDX5 }\end{array}$ \\
\hline GO:0071013 & catalytic step 2 spliceosome & $6 / 119$ & $87 / 19659$ & 0.001 & $\begin{array}{c}\text { HNRNPA1 SYNCRIP HNRNPA2B1 } \\
\text { HNRNPA3 PABPC1 DDX5 }\end{array}$ \\
\hline GO:0044445 & cytosolic part & $9 / 119$ & $250 / 19659$ & 0.001 & $\begin{array}{l}\text { RSL24D1 IKBKB RPL13A PSMD1 } \\
\text { RPL19 BAG6 SNAP47 RPS20 SGTA }\end{array}$ \\
\hline GO:0018995 & host & $4 / 119$ & $29 / 19659$ & 0.001 & KPNB1 XPO1 CD209 CLEC4M \\
\hline GO:0043657 & host cell & $4 / 119$ & $29 / 19659$ & 0.001 & KPNB1 XPO1 CD209 CLEC4M \\
\hline GO:0010494 & cytoplasmic stress granule & $5 / 119$ & $60 / 19659$ & 0.001 & KPNB1 PABPC1 PABPC4 G3BP1 DDX1 \\
\hline GO:0036464 & $\begin{array}{l}\text { cytoplasmic ribonucleoprotein } \\
\text { granule }\end{array}$ & $8 / 119$ & $207 / 19659$ & 0.002 & $\begin{array}{l}\text { PSMC2 KPNB1 PABPC1 PABPC4 } \\
\text { G3BP1 NCL DDX1 PSMA2 }\end{array}$ \\
\hline GO:0044215 & other organism & $4 / 119$ & $35 / 19659$ & 0.002 & KPNB1 XPO1 CD209 CLEC4M \\
\hline GO:0044216 & other organism cell & $4 / 119$ & $35 / 19659$ & 0.002 & KPNB1 XPO1 CD209 CLEC4M \\
\hline GO:0044217 & other organism part & $4 / 119$ & $35 / 19659$ & 0.002 & KPNB1 XPO1 CD209 CLEC4M \\
\hline GO:0033290 & $\begin{array}{l}\text { eukaryotic } 48 \text { s preinitiation } \\
\text { complex }\end{array}$ & $3 / 119$ & $15 / 19659$ & 0.003 & EIF3E EIF3I EIF3F \\
\hline
\end{tabular}




\begin{tabular}{|c|c|c|c|c|c|}
\hline ID & Description & GeneRatio & BgRatio & p.adjust & geneID \\
\hline GO:1904813 & ficolin-1-rich granule lumen & $6 / 119$ & $124 / 19659$ & 0.003 & $\begin{array}{c}\text { PSMC2 KPNB1 FGL2 PPIA PSMA2 } \\
\text { EEF1A1 }\end{array}$ \\
\hline GO:0005852 & $\begin{array}{l}\text { eukaryotic translation initiation } \\
\text { factor } 3 \text { complex }\end{array}$ & $3 / 119$ & $16 / 19659$ & 0.003 & EIF3E EIF3I EIF3F \\
\hline GO:0016282 & $\begin{array}{l}\text { eukaryotic } 43 \text { S preinitiation } \\
\text { complex }\end{array}$ & $3 / 119$ & $17 / 19659$ & 0.003 & EIF3E EIF3I EIF3F \\
\hline GO:0034774 & secretory granule lumen & $9 / 119$ & $321 / 19659$ & 0.003 & $\begin{array}{l}\text { PSMC2 KPNB1 TGFB1 PPIA ANXA2 } \\
\text { PSMD1 SERPING1 PSMA2 EEF1A1 }\end{array}$ \\
\hline GO:0070993 & translation preinitiation complex & $3 / 119$ & $18 / 19659$ & 0.003 & EIF3E EIF3I EIF3F \\
\hline GO:0005925 & focal adhesion & $10 / 119$ & $402 / 19659$ & 0.003 & $\begin{array}{l}\text { PPIA CD9 DPP4 HSPA9 RPL13A } \\
\text { PABPC1 NPM1 G3BP1 RPL19 CAV1 }\end{array}$ \\
\hline GO:0005924 & cell-substrate adherens junction & $10 / 119$ & $405 / 19659$ & 0.003 & $\begin{array}{l}\text { PPIA CD9 DPP4 HSPA9 RPL13A } \\
\text { PABPC1 NPM1 G3BP1 RPL19 CAV1 }\end{array}$ \\
\hline GO:0030055 & cell-substrate junction & $10 / 119$ & $409 / 19659$ & 0.003 & $\begin{array}{l}\text { PPIA CD9 DPP4 HSPA9 RPL13A } \\
\text { PABPC1 NPM1 G3BP1 RPL19 CAV1 }\end{array}$ \\
\hline GO:0060205 & cytoplasmic vesicle lumen & $9 / 119$ & $338 / 19659$ & 0.003 & $\begin{array}{l}\text { PSMC2 KPNB1 TGFB1 PPIA ANXA2 } \\
\text { PSMD1 SERPING1 PSMA2 EEF1A1 }\end{array}$ \\
\hline GO:0031983 & vesicle lumen & $9 / 119$ & $339 / 19659$ & 0.003 & $\begin{array}{l}\text { PSMC2 KPNB1 TGFB1 PPIA ANXA2 } \\
\text { PSMD1 SERPING1 PSMA2 EEF1A1 }\end{array}$ \\
\hline GO:0098978 & glutamatergic synapse & $9 / 119$ & $350 / 19659$ & 0.004 & $\begin{array}{l}\text { PHB STAT3 PPP1CA KPNA2 DNAJB1 } \\
\text { GSK3B CHMP2B SNAP47 ABHD17A }\end{array}$ \\
\hline GO:0000781 & chromosome, telomeric region & $6 / 119$ & $158 / 19659$ & 0.006 & $\begin{array}{l}\text { PPP1CA HNRNPA2B1 PARP1 H2AFY2 } \\
\text { CHEK2 TERF1 }\end{array}$ \\
\hline GO:0043209 & myelin sheath & $6 / 119$ & $162 / 19659$ & 0.006 & $\begin{array}{l}\text { PHB BCL2 ANXA2 ACO2 HSPD1 } \\
\text { NDUFA10 }\end{array}$ \\
\hline GO:0036452 & ESCRT complex & $3 / 119$ & $27 / 19659$ & 0.007 & CHMP4B HGS CHMP2B \\
\hline GO:0005741 & mitochondrial outer membrane & $6 / 119$ & $180 / 19659$ & 0.01 & $\begin{array}{l}\text { PHB BCL2L1 BCL2L2 MCL1 BCL2A1 } \\
\text { BCL2 }\end{array}$ \\
\hline
\end{tabular}

\subsubsection{BIOLOGICAL PROCESS}

\begin{tabular}{|c|c|c|c|c|c|}
\hline ID & Description & GeneRatio & BgRatio & p.adjust & geneID \\
\hline GO:0051701 & interaction with host & $17 / 117$ & $209 / 18493$ & 0 & $\begin{array}{c}\text { KPNB1 TGFB1 SMAD3 ACE2 } \\
\text { CLEC4G CD209 CLEC4M KPNA2 } \\
\text { PPIA BCL2L1 KPNA4 TMPRSS2 } \\
\text { ANPEP DPP4 CHMP4B CHMP2B } \\
\text { CAV1 }\end{array}$ \\
\hline GO:0019058 & viral life cycle & $18 / 117$ & $289 / 18493$ & 0 & $\begin{array}{c}\text { KPNB1 XPO1 ACE2 CLEC4G } \\
\text { CD209 CLEC4M PPIA BCL2 } \\
\text { TMPRSS2 ANPEP DPP4 ANXA2 } \\
\text { CHMP4B PABPC1 PPIH CHMP2B } \\
\text { DDX5 CAV1 }\end{array}$ \\
\hline
\end{tabular}




\begin{tabular}{|c|c|c|c|c|c|}
\hline ID & Description & GeneRatio & BgRatio & p.adjust & geneID \\
\hline GO:0019048 & $\begin{array}{l}\text { modulation by virus of host morphology } \\
\text { or physiology }\end{array}$ & $8 / 117$ & $38 / 18493$ & 0 & $\begin{array}{l}\text { KPNB1 TGFB1 SMAD3 CD209 } \\
\text { CLEC4M KPNA2 BCL2L1 KPNA4 }\end{array}$ \\
\hline GO:0051817 & $\begin{array}{c}\text { modification of morphology or physiology } \\
\text { of other organism involved in symbiotic } \\
\text { interaction }\end{array}$ & $11 / 117$ & $112 / 18493$ & 0 & $\begin{array}{l}\text { KPNB1 JUN TGFB1 SMAD3 } \\
\text { CD209 CLEC4M KPNA2 SFTPD } \\
\text { BCL2L1 KPNA4 ANXA2 }\end{array}$ \\
\hline GO:0042176 & regulation of protein catabolic process & $17 / 117$ & $375 / 18493$ & 0 & $\begin{array}{c}\text { PSMC2 STX5 PHB XPO1 SMAD3 } \\
\text { ANXA2 PSMD1 SNX9 GSK3A } \\
\text { GSK3B RYBP HGS BAG6 CHEK2 } \\
\text { EEF1A1 CAV1 SGTA }\end{array}$ \\
\hline GO:0044003 & $\begin{array}{l}\text { modification by symbiont of host } \\
\text { morphology or physiology }\end{array}$ & $8 / 117$ & $46 / 18493$ & 0 & $\begin{array}{l}\text { KPNB1 TGFB1 SMAD3 CD209 } \\
\text { CLEC4M KPNA2 BCL2L1 KPNA4 }\end{array}$ \\
\hline GO:0009895 & negative regulation of catabolic process & $14 / 117$ & $287 / 18493$ & 0 & $\begin{array}{c}\text { SCFD1 PHB STAT3 SMAD3 MCL1 } \\
\text { BCL2 SYNCRIP ANXA2 CHMP4B } \\
\text { GSK3A PABPC1 RYBP BAG6 } \\
\text { SGTA }\end{array}$ \\
\hline GO:0035821 & $\begin{array}{c}\text { modification of morphology or physiology } \\
\text { of other organism }\end{array}$ & $11 / 117$ & $161 / 18493$ & 0 & $\begin{array}{l}\text { KPNB1 JUN TGFB1 SMAD3 } \\
\text { CD209 CLEC4M KPNA2 SFTPD } \\
\text { BCL2L1 KPNA4 ANXA2 }\end{array}$ \\
\hline GO:0043903 & $\begin{array}{l}\text { regulation of symbiosis, encompassing } \\
\text { mutualism through parasitism }\end{array}$ & $12 / 117$ & $203 / 18493$ & 0 & $\begin{array}{c}\text { JUN SFTPD PPIA BCL2 TMPRSS2 } \\
\text { ANXA2 CHMP4B PABPC1 PPIH } \\
\text { CHMP2B DDX5 CAV1 }\end{array}$ \\
\hline GO:0038034 & signal transduction in absence of ligand & $8 / 117$ & $71 / 18493$ & 0 & $\begin{array}{l}\text { PPP1CA BCL2L1 BCL2L2 MCL1 } \\
\text { BCL2A1 BCL2 GSK3A GSK3B }\end{array}$ \\
\hline GO:0097192 & $\begin{array}{l}\text { extrinsic apoptotic signaling pathway in } \\
\text { absence of ligand }\end{array}$ & $8 / 117$ & $71 / 18493$ & 0 & $\begin{array}{l}\text { PPP1CA BCL2L1 BCL2L2 MCL1 } \\
\text { BCL2A1 BCL2 GSK3A GSK3B }\end{array}$ \\
\hline GO:0006413 & translational initiation & $11 / 117$ & $194 / 18493$ & 0 & $\begin{array}{l}\text { PPP1CA RPL13A EIF3E EIF3I } \\
\text { EIF3F PABPC1 NPM1 RPL19 } \\
\text { DDX1 MIF4GD RPS20 }\end{array}$ \\
\hline GO:0031330 & $\begin{array}{l}\text { negative regulation of cellular catabolic } \\
\text { process }\end{array}$ & $12 / 117$ & $245 / 18493$ & 0 & $\begin{array}{c}\text { SCFD1 STAT3 MCL1 BCL2 } \\
\text { SYNCRIP ANXA2 CHMP4B } \\
\text { GSK3A PABPC1 RYBP BAG6 } \\
\text { SGTA }\end{array}$ \\
\hline GO:0048524 & positive regulation of viral process & $8 / 117$ & $86 / 18493$ & 0 & $\begin{array}{l}\text { JUN PPIA TMPRSS2 ANXA2 } \\
\text { CHMP4B PABPC1 PPIH CHMP2B }\end{array}$ \\
\hline GO:0046718 & viral entry into host cell & $9 / 117$ & $121 / 18493$ & 0 & $\begin{array}{c}\text { ACE2 CLEC4G CD209 CLEC4M } \\
\text { PPIA TMPRSS2 ANPEP DPP4 } \\
\text { CAV1 }\end{array}$ \\
\hline GO: 1903902 & positive regulation of viral life cycle & $7 / 117$ & $60 / 18493$ & 0 & $\begin{array}{c}\text { PPIA TMPRSS2 ANXA2 CHMP4B } \\
\text { PABPC1 PPIH CHMP2B }\end{array}$ \\
\hline GO:2001235 & $\begin{array}{c}\text { positive regulation of apoptotic signaling } \\
\text { pathway }\end{array}$ & $10 / 117$ & $170 / 18493$ & 0 & $\begin{array}{l}\text { PPP1CA SMAD3 BCL2L1 MCL1 } \\
\text { BCL2 PRKRA NMT1 GSK3A } \\
\text { GSK3B CAV1 }\end{array}$ \\
\hline GO:0046794 & transport of virus & $5 / 117$ & $20 / 18493$ & 0 & $\begin{array}{l}\text { KPNB1 XPO1 CD209 CLEC4M } \\
\text { CAV1 }\end{array}$ \\
\hline GO:0030260 & entry into host cell & $9 / 117$ & $133 / 18493$ & 0 & $\begin{array}{c}\text { ACE2 CLEC4G CD209 CLEC4M } \\
\text { PPIA TMPRSS2 ANPEP DPP4 } \\
\text { CAV1 }\end{array}$ \\
\hline GO:0044409 & entry into host & $9 / 117$ & $133 / 18493$ & 0 & $\begin{array}{c}\text { ACE2 CLEC4G CD209 CLEC4M } \\
\text { PPIA TMPRSS2 ANPEP DPP4 } \\
\text { CAV1 }\end{array}$ \\
\hline
\end{tabular}




\begin{tabular}{|c|c|c|c|c|c|}
\hline ID & Description & GeneRatio & BgRatio & p.adjust & geneID \\
\hline GO:0051806 & $\begin{array}{l}\text { entry into cell of other organism involved } \\
\text { in symbiotic interaction }\end{array}$ & $9 / 117$ & $133 / 18493$ & 0 & $\begin{array}{c}\text { ACE2 CLEC4G CD209 CLEC4M } \\
\text { PPIA TMPRSS2 ANPEP DPP4 } \\
\text { CAV1 }\end{array}$ \\
\hline GO:0051828 & $\begin{array}{l}\text { entry into other organism involved in } \\
\text { symbiotic interaction }\end{array}$ & $9 / 117$ & $133 / 18493$ & 0 & $\begin{array}{c}\text { ACE2 CLEC4G CD209 CLEC4M } \\
\text { PPIA TMPRSS2 ANPEP DPP4 } \\
\text { CAV1 }\end{array}$ \\
\hline GO:2001233 & regulation of apoptotic signaling pathway & $14 / 117$ & $391 / 18493$ & 0 & $\begin{array}{c}\text { PPP1CA SMAD3 BCL2L1 BCL2L2 } \\
\text { MCL1 BCL2A1 BCL2 PRKRA } \\
\text { NMT1 NONO GSK3A GSK3B } \\
\text { PARP1 CAV1 }\end{array}$ \\
\hline GO:0097191 & extrinsic apoptotic signaling pathway & $11 / 117$ & $224 / 18493$ & 0 & $\begin{array}{l}\text { PPP1CA TGFB1 SMAD3 BCL2L1 } \\
\text { BCL2L2 MCL1 BCL2A1 BCL2 } \\
\text { GSK3A GSK3B CAV1 }\end{array}$ \\
\hline GO:0050792 & regulation of viral process & $10 / 117$ & $177 / 18493$ & 0 & $\begin{array}{c}\text { JUN PPIA BCL2 TMPRSS2 ANXA2 } \\
\text { CHMP4B PABPC1 PPIH CHMP2B } \\
\text { DDX5 }\end{array}$ \\
\hline GO: 1903900 & regulation of viral life cycle & $9 / 117$ & $140 / 18493$ & 0 & $\begin{array}{c}\text { PPIA BCL2 TMPRSS2 ANXA2 } \\
\text { CHMP4B PABPC1 PPIH CHMP2B } \\
\text { DDX5 }\end{array}$ \\
\hline GO:0097193 & intrinsic apoptotic signaling pathway & $12 / 117$ & $287 / 18493$ & 0 & $\begin{array}{c}\text { BCL2L1 BCL2L2 MCL1 BCL2A1 } \\
\text { BCL2 PRKRA NONO PARP1 BAG6 } \\
\text { CHEK2 DDX5 CAV1 }\end{array}$ \\
\hline GO:0044650 & adhesion of symbiont to host cell & $4 / 117$ & $12 / 18493$ & 0 & ACE2 CD209 CLEC4M SFTPD \\
\hline GO:2001242 & $\begin{array}{l}\text { regulation of intrinsic apoptotic signaling } \\
\text { pathway }\end{array}$ & $9 / 117$ & $161 / 18493$ & 0 & $\begin{array}{l}\text { BCL2L1 BCL2L2 MCL1 BCL2A1 } \\
\text { BCL2 PRKRA NONO PARP1 CAV1 }\end{array}$ \\
\hline GO:0007183 & SMAD protein complex assembly & $4 / 117$ & $13 / 18493$ & 0 & TGFB1 SMAD3 PARP1 FKBP1A \\
\hline GO:0046605 & regulation of centrosome cycle & $6 / 117$ & $56 / 18493$ & 0 & $\begin{array}{c}\text { XPO1 ATF5 CHMP4B KIF11 NPM1 } \\
\text { CHMP2B }\end{array}$ \\
\hline GO:0072593 & reactive oxygen species metabolic process & $11 / 117$ & $276 / 18493$ & 0 & $\begin{array}{c}\text { STAT3 TGFB1 SMAD3 COX2 } \\
\text { ACE2 SFTPD BCL2 HSPD1 GBF1 } \\
\text { DDAH2 CAV1 }\end{array}$ \\
\hline GO:0044766 & multi-organism transport & $5 / 117$ & $33 / 18493$ & 0 & $\begin{array}{l}\text { KPNB1 XPO1 CD209 CLEC4M } \\
\text { CAV1 }\end{array}$ \\
\hline GO: 1902579 & multi-organism localization & $5 / 117$ & $33 / 18493$ & 0 & $\begin{array}{l}\text { KPNB1 XPO1 CD209 CLEC4M } \\
\text { CAV1 }\end{array}$ \\
\hline GO:0044406 & adhesion of symbiont to host & $4 / 117$ & $15 / 18493$ & 0 & ACE2 CD209 CLEC4M SFTPD \\
\hline GO:2000377 & $\begin{array}{l}\text { regulation of reactive oxygen species } \\
\text { metabolic process }\end{array}$ & $9 / 117$ & $185 / 18493$ & 0 & $\begin{array}{c}\text { STAT3 TGFB1 SMAD3 COX2 } \\
\text { ACE2 BCL2 HSPD1 DDAH2 CAV1 }\end{array}$ \\
\hline GO:0075733 & intracellular transport of virus & $4 / 117$ & $17 / 18493$ & 0 & KPNB1 XPO1 CD209 CLEC4M \\
\hline GO:0008630 & $\begin{array}{l}\text { intrinsic apoptotic signaling pathway in } \\
\text { response to DNA damage }\end{array}$ & $7 / 117$ & $102 / 18493$ & 0 & $\begin{array}{l}\text { BCL2L1 BCL2L2 MCL1 BCL2A1 } \\
\text { BCL2 BAG6 CHEK2 }\end{array}$ \\
\hline GO:0051098 & regulation of binding & $12 / 117$ & $369 / 18493$ & 0 & $\begin{array}{c}\text { JUN PPP1CA TGFB1 SMAD3 BCL2 } \\
\text { ANXA2 EIF3E GSK3B PARP1 } \\
\text { FKBP1A MARK3 CAV1 }\end{array}$ \\
\hline GO:0043900 & regulation of multi-organism process & $12 / 117$ & $371 / 18493$ & 0 & $\begin{array}{c}\text { JUN SFTPD PPIA BCL2 TMPRSS2 } \\
\text { ANXA2 CHMP4B PABPC1 PPIH } \\
\text { CHMP2B DDX5 CAV1 }\end{array}$ \\
\hline
\end{tabular}




\begin{tabular}{|c|c|c|c|c|c|}
\hline ID & Description & GeneRatio & BgRatio & p.adjust & geneID \\
\hline GO:0046902 & $\begin{array}{l}\text { regulation of mitochondrial membrane } \\
\text { permeability }\end{array}$ & $6 / 117$ & $73 / 18493$ & 0 & $\begin{array}{l}\text { STAT3 BCL2L1 BCL2 NMT1 } \\
\text { GSK3A GSK3B }\end{array}$ \\
\hline GO:0061614 & $\begin{array}{l}\text { pri-miRNA transcription by RNA } \\
\text { polymerase II }\end{array}$ & $5 / 117$ & $44 / 18493$ & 0.001 & JUN STAT3 TGFB1 SMAD3 DDX5 \\
\hline GO: 1903409 & $\begin{array}{l}\text { reactive oxygen species biosynthetic } \\
\text { process }\end{array}$ & $7 / 117$ & $115 / 18493$ & 0.001 & $\begin{array}{l}\text { STAT3 SMAD3 COX2 HSPD1 } \\
\text { GBF1 DDAH2 CAV1 }\end{array}$ \\
\hline GO:0043902 & $\begin{array}{l}\text { positive regulation of multi-organism } \\
\text { process }\end{array}$ & $8 / 117$ & $162 / 18493$ & 0.001 & $\begin{array}{l}\text { JUN PPIA TMPRSS2 ANXA2 } \\
\text { CHMP4B PABPC1 PPIH CHMP2B }\end{array}$ \\
\hline GO:0006446 & regulation of translational initiation & $6 / 117$ & $77 / 18493$ & 0.001 & $\begin{array}{l}\text { PPP1CA RPL13A EIF3E NPM1 } \\
\text { DDX1 MIF4GD }\end{array}$ \\
\hline GO:0030193 & regulation of blood coagulation & $6 / 117$ & $77 / 18493$ & 0.001 & $\begin{array}{l}\text { CD9 CEACAM1 ANXA2 } \\
\text { SERPING1 VKORC1 CAV1 }\end{array}$ \\
\hline GO: 1900046 & regulation of hemostasis & $6 / 117$ & $77 / 18493$ & 0.001 & $\begin{array}{l}\text { CD9 CEACAM1 ANXA2 } \\
\text { SERPING1 VKORC1 CAV1 }\end{array}$ \\
\hline GO:0019079 & viral genome replication & $7 / 117$ & $117 / 18493$ & 0.001 & $\begin{array}{l}\text { CD209 CLEC4M PPIA BCL2 } \\
\text { PABPC1 PPIH DDX5 }\end{array}$ \\
\hline GO:0006457 & protein folding & $9 / 117$ & $218 / 18493$ & 0.001 & $\begin{array}{l}\text { PPIA DNAJB1 HSPD1 HSPA9 } \\
\text { FKBP1A PPIG PPIH PFDN5 SGTA }\end{array}$ \\
\hline GO:0035196 & $\begin{array}{l}\text { production of miRNAs involved in gene } \\
\text { silencing by miRNA }\end{array}$ & $5 / 117$ & $46 / 18493$ & 0.001 & $\begin{array}{l}\text { TGFB1 SMAD3 PRKRA } \\
\text { HNRNPA2B1 DDX5 }\end{array}$ \\
\hline GO:0008637 & apoptotic mitochondrial changes & $7 / 117$ & $121 / 18493$ & 0.001 & $\begin{array}{l}\text { JUN BCL2L1 BCL2 HSPD1 NMT1 } \\
\text { GSK3A GSK3B }\end{array}$ \\
\hline GO:0090559 & regulation of membrane permeability & $6 / 117$ & $81 / 18493$ & 0.001 & $\begin{array}{l}\text { STAT3 BCL2L1 BCL2 NMT1 } \\
\text { GSK3A GSK3B }\end{array}$ \\
\hline GO:0050818 & regulation of coagulation & $6 / 117$ & $82 / 18493$ & 0.001 & $\begin{array}{l}\text { CD9 CEACAM1 ANXA2 } \\
\text { SERPING1 VKORC1 CAV1 }\end{array}$ \\
\hline GO:0051656 & establishment of organelle localization & $13 / 117$ & $491 / 18493$ & 0.001 & $\begin{array}{l}\text { SCFD1 YKT6 KPNB1 STX5 XPO1 } \\
\text { CHMP4B GBF1 GSK3B NPM1 } \\
\text { CHMP2B SNAP47 TERF1 DCTN2 }\end{array}$ \\
\hline GO:0031050 & dsRNA processing & $5 / 117$ & $49 / 18493$ & 0.001 & $\begin{array}{l}\text { TGFB1 SMAD3 PRKRA } \\
\text { HNRNPA2B1 DDX5 }\end{array}$ \\
\hline GO:0070918 & $\begin{array}{c}\text { production of small RNA involved in gene } \\
\text { silencing by RNA }\end{array}$ & $5 / 117$ & $49 / 18493$ & 0.001 & $\begin{array}{l}\text { TGFB1 SMAD3 PRKRA } \\
\text { HNRNPA2B1 DDX5 }\end{array}$ \\
\hline GO:0045862 & positive regulation of proteolysis & $11 / 117$ & $352 / 18493$ & 0.001 & $\begin{array}{c}\text { PSMC2 PHB STAT3 SMAD3 } \\
\text { HSPD1 SNX9 GSK3A GSK3B } \\
\text { BAG6 CAV1 SGTA }\end{array}$ \\
\hline GO:2000209 & regulation of anoikis & $4 / 117$ & $24 / 18493$ & 0.001 & MCL1 BCL2 CHEK2 CAV1 \\
\hline GO:0006913 & nucleocytoplasmic transport & $11 / 117$ & $357 / 18493$ & 0.001 & $\begin{array}{c}\text { KPNB1 STAT3 XPO1 TGFB1 } \\
\text { SMAD3 HNRNPA1 KPNA2 KPNA4 } \\
\text { HNRNPA2B1 GSK3B NPM1 }\end{array}$ \\
\hline GO:0019054 & modulation by virus of host process & $4 / 117$ & $25 / 18493$ & 0.001 & KPNB1 KPNA2 BCL2L1 KPNA4 \\
\hline GO:0051169 & nuclear transport & $11 / 117$ & $360 / 18493$ & 0.001 & $\begin{array}{c}\text { KPNB1 STAT3 XPO1 TGFB1 } \\
\text { SMAD3 HNRNPA1 KPNA2 KPNA4 } \\
\text { HNRNPA2B1 GSK3B NPM1 }\end{array}$ \\
\hline
\end{tabular}




\begin{tabular}{|c|c|c|c|c|c|}
\hline ID & Description & GeneRatio & BgRatio & p.adjust & geneID \\
\hline GO:1901214 & regulation of neuron death & $10 / 117$ & $296 / 18493$ & 0.001 & $\begin{array}{c}\text { JUN STAT3 BCL2L1 MCL1 BCL2 } \\
\text { HSPD1 CHMP4B NONO GSK3B } \\
\text { PARP1 }\end{array}$ \\
\hline GO:0051099 & positive regulation of binding & $8 / 117$ & $181 / 18493$ & 0.001 & $\begin{array}{l}\text { TGFB1 ANXA2 EIF3E GSK3B } \\
\text { PARP1 FKBP1A MARK3 CAV1 }\end{array}$ \\
\hline GO:0042026 & protein refolding & $4 / 117$ & $26 / 18493$ & 0.001 & HSPD1 HSPA9 FKBP1A PPIG \\
\hline GO:0061041 & regulation of wound healing & $7 / 117$ & $134 / 18493$ & 0.001 & $\begin{array}{l}\text { SMAD3 CD9 CEACAM1 ANXA2 } \\
\text { SERPING1 VKORC1 CAV1 }\end{array}$ \\
\hline GO:1902895 & $\begin{array}{l}\text { positive regulation of pri-miRNA } \\
\text { transcription by RNA polymerase II }\end{array}$ & $4 / 117$ & $27 / 18493$ & 0.001 & JUN STAT3 TGFB1 SMAD3 \\
\hline GO:0006402 & mRNA catabolic process & $10 / 117$ & $307 / 18493$ & 0.001 & $\begin{array}{l}\text { XPO1 SYNCRIP RPL13A EIF3E } \\
\text { PABPC1 PABPC4 NPM1 RPL19 } \\
\text { RPS20 DDX5 }\end{array}$ \\
\hline GO:0019062 & virion attachment to host cell & $3 / 117$ & $10 / 18493$ & 0.001 & ACE2 CD209 CLEC4M \\
\hline GO:0042110 & $\mathrm{T}$ cell activation & $12 / 117$ & $451 / 18493$ & 0.001 & $\begin{array}{l}\text { STAT3 TGFB1 SMAD3 CLEC4G } \\
\text { CD209 SFTPD BCL2 DPP4 } \\
\text { CEACAM1 HSPD1 FKBP1A CAV1 }\end{array}$ \\
\hline GO:1903426 & $\begin{array}{l}\text { regulation of reactive oxygen species } \\
\text { biosynthetic process }\end{array}$ & $6 / 117$ & $94 / 18493$ & 0.001 & $\begin{array}{c}\text { STAT3 SMAD3 COX2 HSPD1 } \\
\text { DDAH2 CAV1 }\end{array}$ \\
\hline GO:2001244 & $\begin{array}{l}\text { positive regulation of intrinsic apoptotic } \\
\text { signaling pathway }\end{array}$ & $5 / 117$ & $57 / 18493$ & 0.001 & $\begin{array}{l}\text { BCL2L1 MCL1 BCL2 PRKRA } \\
\text { CAV1 }\end{array}$ \\
\hline GO:0034248 & $\begin{array}{l}\text { regulation of cellular amide metabolic } \\
\text { process }\end{array}$ & $12 / 117$ & $453 / 18493$ & 0.001 & $\begin{array}{c}\text { STAT3 PPP1CA SYNCRIP RPL13A } \\
\text { EIF3E GSK3A PABPC1 NPM1 NCL } \\
\text { DDX1 MIF4GD EEF1A1 }\end{array}$ \\
\hline GO:0016050 & vesicle organization & $10 / 117$ & $314 / 18493$ & 0.001 & $\begin{array}{c}\text { SCFD1 STX5 BCL2 ANXA2 } \\
\text { CHMP4B GBF1 HGS CHMP2B } \\
\text { SNAP47 CAV1 }\end{array}$ \\
\hline GO:0044068 & $\begin{array}{c}\text { modulation by symbiont of host cellular } \\
\text { process }\end{array}$ & $4 / 117$ & $29 / 18493$ & 0.001 & KPNB1 KPNA2 BCL2L1 KPNA4 \\
\hline GO:2001243 & $\begin{array}{l}\text { negative regulation of intrinsic apoptotic } \\
\text { signaling pathway }\end{array}$ & $6 / 117$ & $96 / 18493$ & 0.001 & $\begin{array}{l}\text { BCL2L1 BCL2L2 MCL1 BCL2A1 } \\
\text { BCL2 NONO }\end{array}$ \\
\hline GO:0090150 & $\begin{array}{l}\text { establishment of protein localization to } \\
\text { membrane }\end{array}$ & $10 / 117$ & $317 / 18493$ & 0.001 & $\begin{array}{c}\text { BCL2 RPL13A NMT1 CHMP4B } \\
\text { SRP54 RPL19 BAG6 SNAP47 } \\
\text { RPS20 SGTA }\end{array}$ \\
\hline GO:0008380 & RNA splicing & $12 / 117$ & $463 / 18493$ & 0.001 & $\begin{array}{c}\text { HNRNPA1 ZCRB1 SYNCRIP } \\
\text { PTBP1 HNRNPA2B1 HNRNPA3 } \\
\text { NONO PABPC1 DDX1 PPIG PPIH } \\
\text { DDX5 }\end{array}$ \\
\hline GO:0090307 & mitotic spindle assembly & $5 / 117$ & $60 / 18493$ & 0.001 & $\begin{array}{c}\text { KPNB1 CHMP4B KIF11 CHMP2B } \\
\text { CHEK2 }\end{array}$ \\
\hline GO:0070972 & $\begin{array}{l}\text { protein localization to endoplasmic } \\
\text { reticulum }\end{array}$ & $7 / 117$ & $146 / 18493$ & 0.002 & $\begin{array}{l}\text { RPL13A CHMP4B GBF1 SRP54 } \\
\text { RPL19 RPS20 SGTA }\end{array}$ \\
\hline GO:0002183 & cytoplasmic translational initiation & $4 / 117$ & $31 / 18493$ & 0.002 & RPL13A EIF3E EIF3I EIF3F \\
\hline GO:1901215 & negative regulation of neuron death & $8 / 117$ & $201 / 18493$ & 0.002 & $\begin{array}{l}\text { JUN STAT3 BCL2L1 BCL2 HSPD1 } \\
\text { CHMP4B NONO GSK3B }\end{array}$ \\
\hline GO:0061025 & membrane fusion & $7 / 117$ & $149 / 18493$ & 0.002 & $\begin{array}{c}\text { YKT6 STX5 PPIA CD9 ANXA2 } \\
\text { CHMP2B SNAP47 }\end{array}$ \\
\hline
\end{tabular}




\begin{tabular}{|c|c|c|c|c|c|}
\hline ID & Description & GeneRatio & BgRatio & p.adjust & geneID \\
\hline GO:1903311 & regulation of mRNA metabolic process & $9 / 117$ & $264 / 18493$ & 0.002 & $\begin{array}{c}\text { XPO1 HNRNPA1 SYNCRIP PTBP1 } \\
\text { HNRNPA2B1 PABPC1 PABPC4 } \\
\text { NPM1 DDX5 }\end{array}$ \\
\hline GO: 1901030 & $\begin{array}{l}\text { positive regulation of mitochondrial outer } \\
\text { membrane permeabilization involved in } \\
\text { apoptotic signaling pathway }\end{array}$ & $4 / 117$ & $32 / 18493$ & 0.002 & BCL2 NMT1 GSK3A GSK3B \\
\hline GO: 1901800 & $\begin{array}{l}\text { positive regulation of proteasomal protein } \\
\text { catabolic process }\end{array}$ & $6 / 117$ & $103 / 18493$ & 0.002 & $\begin{array}{c}\text { PSMC2 GSK3A GSK3B BAG6 } \\
\text { CAV1 SGTA }\end{array}$ \\
\hline GO:0070997 & neuron death & $10 / 117$ & $335 / 18493$ & 0.002 & $\begin{array}{c}\text { JUN STAT3 BCL2L1 MCL1 BCL2 } \\
\text { HSPD1 CHMP4B NONO GSK3B } \\
\text { PARP1 }\end{array}$ \\
\hline GO:0034504 & protein localization to nucleus & $9 / 117$ & $269 / 18493$ & 0.002 & $\begin{array}{c}\text { KPNB1 STAT3 XPO1 TGFB1 } \\
\text { SMAD3 KPNA2 KPNA4 GSK3B } \\
\text { PARP1 }\end{array}$ \\
\hline GO:0006401 & RNA catabolic process & $10 / 117$ & $340 / 18493$ & 0.002 & $\begin{array}{l}\text { XPO1 SYNCRIP RPL13A EIF3E } \\
\text { PABPC1 PABPC4 NPM1 RPL19 } \\
\text { RPS20 DDX5 }\end{array}$ \\
\hline GO:0043276 & anoikis & $4 / 117$ & $34 / 18493$ & 0.002 & MCL1 BCL2 CHEK2 CAV1 \\
\hline GO:0045732 & $\begin{array}{l}\text { positive regulation of protein catabolic } \\
\text { process }\end{array}$ & $8 / 117$ & $212 / 18493$ & 0.002 & $\begin{array}{l}\text { PSMC2 STX5 SNX9 GSK3A } \\
\text { GSK3B BAG6 CAV1 SGTA }\end{array}$ \\
\hline GO:0022618 & ribonucleoprotein complex assembly & $9 / 117$ & $275 / 18493$ & 0.002 & $\begin{array}{l}\text { RSL24D1 RPL13A EIF3E EIF3I } \\
\text { EIF3F NPM1 G3BP1 G3BP2 DDX1 }\end{array}$ \\
\hline GO:0007052 & mitotic spindle organization & $6 / 117$ & $108 / 18493$ & 0.002 & $\begin{array}{l}\text { KPNB1 CHMP4B KIF11 CHMP2B } \\
\text { CHEK2 DCTN2 }\end{array}$ \\
\hline GO:0032885 & $\begin{array}{c}\text { regulation of polysaccharide biosynthetic } \\
\text { process }\end{array}$ & $4 / 117$ & $35 / 18493$ & 0.002 & PPP1CA TGFB1 GSK3A GSK3B \\
\hline GO:1903034 & regulation of response to wounding & $7 / 117$ & $160 / 18493$ & 0.002 & $\begin{array}{l}\text { SMAD3 CD9 CEACAM1 ANXA2 } \\
\text { SERPING1 VKORC1 CAV1 }\end{array}$ \\
\hline GO:0001732 & $\begin{array}{l}\text { formation of cytoplasmic translation } \\
\text { initiation complex }\end{array}$ & $3 / 117$ & $14 / 18493$ & 0.003 & EIF3E EIF3I EIF3F \\
\hline GO:1904294 & positive regulation of ERAD pathway & $3 / 117$ & $14 / 18493$ & 0.003 & BAG6 CAV1 SGTA \\
\hline GO:1902893 & $\begin{array}{l}\text { regulation of pri-miRNA transcription by } \\
\text { RNA polymerase II }\end{array}$ & $4 / 117$ & $37 / 18493$ & 0.003 & JUN STAT3 TGFB1 SMAD3 \\
\hline GO:0062012 & $\begin{array}{c}\text { regulation of small molecule metabolic } \\
\text { process }\end{array}$ & $10 / 117$ & $355 / 18493$ & 0.003 & $\begin{array}{l}\text { KPNB1 STAT3 PPP1CA TGFB1 } \\
\text { COX2 CEACAM1 GSK3A GSK3B } \\
\text { PARP1 CAV1 }\end{array}$ \\
\hline GO:0061045 & negative regulation of wound healing & $5 / 117$ & $72 / 18493$ & 0.003 & $\begin{array}{l}\text { SMAD3 CD9 CEACAM1 ANXA2 } \\
\text { SERPING1 }\end{array}$ \\
\hline GO:0071826 & $\begin{array}{c}\text { ribonucleoprotein complex subunit } \\
\text { organization }\end{array}$ & $9 / 117$ & $289 / 18493$ & 0.003 & $\begin{array}{l}\text { RSL24D1 RPL13A EIF3E EIF3I } \\
\text { EIF3F NPM1 G3BP1 G3BP2 DDX1 }\end{array}$ \\
\hline GO:0045047 & protein targeting to ER & $6 / 117$ & $117 / 18493$ & 0.003 & $\begin{array}{l}\text { RPL13A CHMP4B SRP54 RPL19 } \\
\text { RPS20 SGTA }\end{array}$ \\
\hline GO: 1903052 & $\begin{array}{l}\text { positive regulation of proteolysis involved } \\
\text { in cellular protein catabolic process }\end{array}$ & $6 / 117$ & $118 / 18493$ & 0.003 & $\begin{array}{c}\text { PSMC2 GSK3A GSK3B BAG6 } \\
\text { CAV1 SGTA }\end{array}$ \\
\hline GO:0000413 & protein peptidyl-prolyl isomerization & $4 / 117$ & $39 / 18493$ & 0.003 & PPIA FKBP1A PPIG PPIH \\
\hline
\end{tabular}




\begin{tabular}{|c|c|c|c|c|c|}
\hline ID & Description & GeneRatio & BgRatio & p.adjust & geneID \\
\hline GO:0007098 & centrosome cycle & $6 / 117$ & $119 / 18493$ & 0.003 & $\begin{array}{l}\text { XPO1 ATF5 CHMP4B KIF11 NPM1 } \\
\text { CHMP2B }\end{array}$ \\
\hline GO:0006458 & 'de novo' protein folding & $4 / 117$ & $40 / 18493$ & 0.003 & DNAJB1 HSPD1 HSPA9 FKBP1A \\
\hline GO:0032881 & $\begin{array}{l}\text { regulation of polysaccharide metabolic } \\
\text { process }\end{array}$ & $4 / 117$ & $40 / 18493$ & 0.003 & PPP1CA TGFB1 GSK3A GSK3B \\
\hline GO:0032434 & $\begin{array}{l}\text { regulation of proteasomal ubiquitin- } \\
\text { dependent protein catabolic process }\end{array}$ & $6 / 117$ & $121 / 18493$ & 0.003 & $\begin{array}{c}\text { GSK3A GSK3B RYBP BAG6 CAV1 } \\
\text { SGTA }\end{array}$ \\
\hline GO:0072599 & $\begin{array}{l}\text { establishment of protein localization to } \\
\text { endoplasmic reticulum }\end{array}$ & $6 / 117$ & $121 / 18493$ & 0.003 & $\begin{array}{l}\text { RPL13A CHMP4B SRP54 RPL19 } \\
\text { RPS20 SGTA }\end{array}$ \\
\hline GO:0070507 & $\begin{array}{c}\text { regulation of microtubule cytoskeleton } \\
\text { organization }\end{array}$ & $7 / 117$ & $175 / 18493$ & 0.003 & $\begin{array}{l}\text { XPO1 ATF5 CHMP4B KIF11 } \\
\text { GSK3B NPM1 CHMP2B }\end{array}$ \\
\hline GO: 1901028 & $\begin{array}{l}\text { regulation of mitochondrial outer } \\
\text { membrane permeabilization involved in } \\
\text { apoptotic signaling pathway }\end{array}$ & $4 / 117$ & $41 / 18493$ & 0.004 & BCL2 NMT1 GSK3A GSK3B \\
\hline GO:0000377 & $\begin{array}{l}\text { RNA splicing, via transesterification } \\
\text { reactions with bulged adenosine as } \\
\text { nucleophile }\end{array}$ & $10 / 117$ & $374 / 18493$ & 0.004 & $\begin{array}{l}\text { HNRNPA1 ZCRB1 SYNCRIP } \\
\text { PTBP1 HNRNPA2B1 HNRNPA3 } \\
\text { PABPC1 DDX1 PPIH DDX5 }\end{array}$ \\
\hline GO:0000398 & mRNA splicing, via spliceosome & $10 / 117$ & $374 / 18493$ & 0.004 & $\begin{array}{l}\text { HNRNPA1 ZCRB1 SYNCRIP } \\
\text { PTBP1 HNRNPA2B1 HNRNPA3 } \\
\text { PABPC1 DDX1 PPIH DDX5 }\end{array}$ \\
\hline GO:0051170 & import into nucleus & $7 / 117$ & $178 / 18493$ & 0.004 & $\begin{array}{l}\text { KPNB1 STAT3 TGFB1 SMAD3 } \\
\text { HNRNPA1 KPNA2 KPNA4 }\end{array}$ \\
\hline GO:0010498 & proteasomal protein catabolic process & $11 / 117$ & $452 / 18493$ & 0.004 & $\begin{array}{c}\text { PSMC2 SKP2 PSMD1 GSK3A } \\
\text { GSK3B RYBP BAG6 PSMA2 CAV1 } \\
\text { UBE2I SGTA }\end{array}$ \\
\hline GO:0010824 & regulation of centrosome duplication & $4 / 117$ & $42 / 18493$ & 0.004 & XPO1 CHMP4B NPM1 CHMP2B \\
\hline GO:0000375 & $\begin{array}{l}\text { RNA splicing, via transesterification } \\
\text { reactions }\end{array}$ & $10 / 117$ & $377 / 18493$ & 0.004 & $\begin{array}{l}\text { HNRNPA1 ZCRB1 SYNCRIP } \\
\text { PTBP1 HNRNPA2B1 HNRNPA3 } \\
\text { PABPC1 DDX1 PPIH DDX5 }\end{array}$ \\
\hline GO:0061136 & $\begin{array}{l}\text { regulation of proteasomal protein } \\
\text { catabolic process }\end{array}$ & $7 / 117$ & $179 / 18493$ & 0.004 & $\begin{array}{c}\text { PSMC2 GSK3A GSK3B RYBP } \\
\text { BAG6 CAV1 SGTA }\end{array}$ \\
\hline GO:0033143 & $\begin{array}{l}\text { regulation of intracellular steroid hormone } \\
\text { receptor signaling pathway }\end{array}$ & $5 / 117$ & $79 / 18493$ & 0.004 & PHB SKP2 PARP1 DDX5 UBE2I \\
\hline GO:1903362 & $\begin{array}{c}\text { regulation of cellular protein catabolic } \\
\text { process }\end{array}$ & $8 / 117$ & $242 / 18493$ & 0.004 & $\begin{array}{l}\text { PSMC2 ANXA2 GSK3A GSK3B } \\
\text { RYBP BAG6 CAV1 SGTA }\end{array}$ \\
\hline GO:0045912 & $\begin{array}{l}\text { negative regulation of carbohydrate } \\
\text { metabolic process }\end{array}$ & $4 / 117$ & $43 / 18493$ & 0.004 & STAT3 TGFB1 GSK3A GSK3B \\
\hline GO:0033145 & $\begin{array}{l}\text { positive regulation of intracellular steroid } \\
\text { hormone receptor signaling pathway }\end{array}$ & $3 / 117$ & $17 / 18493$ & 0.004 & SKP2 PARP1 UBE2I \\
\hline GO:2000811 & negative regulation of anoikis & $3 / 117$ & $17 / 18493$ & 0.004 & MCL1 BCL2 CAV1 \\
\hline GO:0042177 & $\begin{array}{c}\text { negative regulation of protein catabolic } \\
\text { process }\end{array}$ & $6 / 117$ & $128 / 18493$ & 0.004 & $\begin{array}{c}\text { PHB SMAD3 ANXA2 RYBP BAG6 } \\
\text { SGTA }\end{array}$ \\
\hline GO:0043112 & receptor metabolic process & $7 / 117$ & $184 / 18493$ & 0.004 & $\begin{array}{l}\text { TGFB1 ACE2 CD9 CEACAM1 } \\
\text { ANXA2 CAMLG CAV1 }\end{array}$ \\
\hline
\end{tabular}




\begin{tabular}{|c|c|c|c|c|c|}
\hline ID & Description & GeneRatio & BgRatio & p.adjust & geneID \\
\hline GO: 1902850 & $\begin{array}{l}\text { microtubule cytoskeleton organization } \\
\text { involved in mitosis }\end{array}$ & $6 / 117$ & $129 / 18493$ & 0.004 & $\begin{array}{l}\text { KPNB1 CHMP4B KIF11 CHMP2B } \\
\text { CHEK2 DCTN2 }\end{array}$ \\
\hline GO:0009615 & response to virus & $9 / 117$ & $315 / 18493$ & 0.004 & $\begin{array}{l}\text { IRF3 BCL2L1 BCL2 SKP2 PRKRA } \\
\text { IKBKB GBF1 DDX1 PSMA2 }\end{array}$ \\
\hline GO:0006914 & autophagy & $11 / 117$ & $466 / 18493$ & 0.004 & $\begin{array}{c}\text { SCFD1 TFEB ACBD5 MCL1 BCL2 } \\
\text { CHMP4B GSK3A HGS CHMP2B } \\
\text { EEF1A1 ATP6V1G1 }\end{array}$ \\
\hline GO:0061919 & process utilizing autophagic mechanism & $11 / 117$ & $466 / 18493$ & 0.004 & $\begin{array}{c}\text { SCFD1 TFEB ACBD5 MCL1 BCL2 } \\
\text { CHMP4B GSK3A HGS CHMP2B } \\
\text { EEF1A1 ATP6V1G1 }\end{array}$ \\
\hline GO:0043161 & $\begin{array}{l}\text { proteasome-mediated ubiquitin-dependent } \\
\text { protein catabolic process }\end{array}$ & $10 / 117$ & $389 / 18493$ & 0.004 & $\begin{array}{c}\text { SKP2 PSMD1 GSK3A GSK3B } \\
\text { RYBP BAG6 PSMA2 CAV1 UBE2I } \\
\text { SGTA }\end{array}$ \\
\hline GO:0007006 & mitochondrial membrane organization & $6 / 117$ & $131 / 18493$ & 0.004 & $\begin{array}{l}\text { STAT3 BCL2L1 BCL2 NMT1 } \\
\text { GSK3A GSK3B }\end{array}$ \\
\hline GO:0008631 & $\begin{array}{l}\text { intrinsic apoptotic signaling pathway in } \\
\text { response to oxidative stress }\end{array}$ & $4 / 117$ & $45 / 18493$ & 0.004 & MCL1 BCL2 NONO PARP1 \\
\hline GO:1903035 & $\begin{array}{l}\text { negative regulation of response to } \\
\text { wounding }\end{array}$ & $5 / 117$ & $84 / 18493$ & 0.004 & $\begin{array}{l}\text { SMAD3 CD9 CEACAM1 ANXA2 } \\
\text { SERPING1 }\end{array}$ \\
\hline GO:0031023 & $\begin{array}{c}\text { microtubule organizing center } \\
\text { organization }\end{array}$ & $6 / 117$ & $133 / 18493$ & 0.005 & $\begin{array}{l}\text { XPO1 ATF5 CHMP4B KIF11 NPM1 } \\
\text { CHMP2B }\end{array}$ \\
\hline GO:2001239 & $\begin{array}{l}\text { regulation of extrinsic apoptotic signaling } \\
\text { pathway in absence of ligand }\end{array}$ & $4 / 117$ & $46 / 18493$ & 0.005 & PPP1CA BCL2L1 MCL1 BCL2 \\
\hline GO:0046677 & response to antibiotic & $9 / 117$ & $323 / 18493$ & 0.005 & $\begin{array}{c}\text { PHB JUN STAT3 HNRNPA1 } \\
\text { BCL2L1 BCL2 KPNA4 HSPD1 } \\
\text { VKORC1 }\end{array}$ \\
\hline GO:0035722 & $\begin{array}{l}\text { interleukin-12-mediated signaling } \\
\text { pathway }\end{array}$ & $4 / 117$ & $47 / 18493$ & 0.005 & PPIA ANXA2 HNRNPA2B1 HSPA9 \\
\hline GO:0051205 & protein insertion into membrane & $4 / 117$ & $47 / 18493$ & 0.005 & BCL2 NMT1 BAG6 SGTA \\
\hline GO:0007159 & leukocyte cell-cell adhesion & $9 / 117$ & $327 / 18493$ & 0.005 & $\begin{array}{c}\text { TGFB1 CLEC4G CD209 CLEC4M } \\
\text { SFTPD DPP4 CEACAM1 HSPD1 } \\
\text { CAV1 }\end{array}$ \\
\hline GO:0001889 & liver development & $6 / 117$ & $137 / 18493$ & 0.005 & $\begin{array}{l}\text { JUN TGFB1 SMAD3 CEACAM1 } \\
\text { ACO2 RPL19 }\end{array}$ \\
\hline GO:0006417 & regulation of translation & $10 / 117$ & $402 / 18493$ & 0.005 & $\begin{array}{c}\text { STAT3 PPP1CA SYNCRIP RPL13A } \\
\text { EIF3E PABPC1 NPM1 NCL DDX1 } \\
\text { MIF4GD }\end{array}$ \\
\hline GO:0035304 & regulation of protein dephosphorylation & $6 / 117$ & $138 / 18493$ & 0.005 & $\begin{array}{c}\text { TGFB1 IKBKB PTBP1 GSK3B } \\
\text { FKBP1A RCAN3 }\end{array}$ \\
\hline GO:1903364 & $\begin{array}{l}\text { positive regulation of cellular protein } \\
\text { catabolic process }\end{array}$ & $6 / 117$ & $138 / 18493$ & 0.005 & $\begin{array}{c}\text { PSMC2 GSK3A GSK3B BAG6 } \\
\text { CAV1 SGTA }\end{array}$ \\
\hline GO:0022613 & ribonucleoprotein complex biogenesis & $11 / 117$ & $485 / 18493$ & 0.005 & $\begin{array}{l}\text { RSL24D1 XPO1 RPL13A EIF3E } \\
\text { EIF3I EIF3F NPM1 G3BP1 G3BP2 } \\
\text { DDX1 LAS1L }\end{array}$ \\
\hline GO:0071349 & cellular response to interleukin- 12 & $4 / 117$ & $49 / 18493$ & 0.005 & PPIA ANXA2 HNRNPA2B1 HSPA9 \\
\hline
\end{tabular}




\begin{tabular}{|c|c|c|c|c|c|}
\hline ID & Description & GeneRatio & BgRatio & p.adjust & geneID \\
\hline GO:0009896 & positive regulation of catabolic process & $10 / 117$ & $407 / 18493$ & 0.005 & $\begin{array}{c}\text { PSMC2 TFEB STX5 SNX9 GSK3A } \\
\text { GSK3B PABPC1 BAG6 CAV1 } \\
\text { SGTA }\end{array}$ \\
\hline GO:0061008 & hepaticobiliary system development & $6 / 117$ & $140 / 18493$ & 0.005 & $\begin{array}{l}\text { JUN TGFB1 SMAD3 CEACAM1 } \\
\text { ACO2 RPL19 }\end{array}$ \\
\hline GO:0045069 & regulation of viral genome replication & $5 / 117$ & $90 / 18493$ & 0.005 & PPIA BCL2 PABPC1 PPIH DDX5 \\
\hline GO:0051236 & establishment of RNA localization & $7 / 117$ & $198 / 18493$ & 0.005 & $\begin{array}{l}\text { XPO1 HNRNPA1 HNRNPA2B1 } \\
\text { HNRNPA3 NPM1 G3BP2 TERF1 }\end{array}$ \\
\hline GO:0070671 & response to interleukin- 12 & $4 / 117$ & $50 / 18493$ & 0.006 & PPIA ANXA2 HNRNPA2B1 HSPA9 \\
\hline GO:0043523 & regulation of neuron apoptotic process & $7 / 117$ & $202 / 18493$ & 0.006 & $\begin{array}{l}\text { JUN BCL2L1 MCL1 BCL2 HSPD1 } \\
\text { NONO PARP1 }\end{array}$ \\
\hline GO:0071359 & cellular response to dsRNA & $3 / 117$ & $21 / 18493$ & 0.006 & IRF3 NPM1 CAV1 \\
\hline GO:1904886 & $\begin{array}{l}\text { beta-catenin destruction complex } \\
\text { disassembly }\end{array}$ & $3 / 117$ & $21 / 18493$ & 0.006 & PPP1CA GSK3B CAV1 \\
\hline GO:0097345 & $\begin{array}{l}\text { mitochondrial outer membrane } \\
\text { permeabilization }\end{array}$ & $4 / 117$ & $51 / 18493$ & 0.006 & BCL2 NMT1 GSK3A GSK3B \\
\hline GO:0002181 & cytoplasmic translation & $5 / 117$ & $93 / 18493$ & 0.006 & RPL13A EIF3E EIF3I EIF3F RPL19 \\
\hline GO:0032886 & regulation of microtubule-based process & $7 / 117$ & $204 / 18493$ & 0.006 & $\begin{array}{l}\text { XPO1 ATF5 CHMP4B KIF11 } \\
\text { GSK3B NPM1 CHMP2B }\end{array}$ \\
\hline GO:0030195 & negative regulation of blood coagulation & $4 / 117$ & $52 / 18493$ & 0.006 & $\begin{array}{l}\text { CD9 CEACAM1 ANXA2 } \\
\text { SERPING1 }\end{array}$ \\
\hline GO: 1900047 & negative regulation of hemostasis & $4 / 117$ & $52 / 18493$ & 0.006 & $\begin{array}{l}\text { CD9 CEACAM1 ANXA2 } \\
\text { SERPING1 }\end{array}$ \\
\hline GO:0032092 & positive regulation of protein binding & $5 / 117$ & $94 / 18493$ & 0.006 & $\begin{array}{c}\text { ANXA2 GSK3B FKBP1A MARK3 } \\
\text { CAV1 }\end{array}$ \\
\hline GO:0035303 & regulation of dephosphorylation & $7 / 117$ & $206 / 18493$ & 0.006 & $\begin{array}{l}\text { TGFB1 SMAD3 IKBKB PTBP1 } \\
\text { GSK3B FKBP1A RCAN3 }\end{array}$ \\
\hline GO:2000058 & $\begin{array}{l}\text { regulation of ubiquitin-dependent protein } \\
\text { catabolic process }\end{array}$ & $6 / 117$ & $147 / 18493$ & 0.006 & $\begin{array}{c}\text { GSK3A GSK3B RYBP BAG6 CAV1 } \\
\text { SGTA }\end{array}$ \\
\hline GO:2000637 & $\begin{array}{l}\text { positive regulation of gene silencing by } \\
\text { miRNA }\end{array}$ & $3 / 117$ & $22 / 18493$ & 0.006 & STAT3 TGFB1 DDX5 \\
\hline GO:0010332 & response to gamma radiation & $4 / 117$ & $53 / 18493$ & 0.006 & BCL2L1 BCL2 PARP1 CHEK2 \\
\hline GO:0043331 & response to dsRNA & $4 / 117$ & $53 / 18493$ & 0.006 & IRF3 NPM1 DDX1 CAV1 \\
\hline GO:0016197 & endosomal transport & $7 / 117$ & $208 / 18493$ & 0.007 & $\begin{array}{c}\text { YKT6 STX5 CHMP4B GBF1 SNX9 } \\
\text { HGS CHMP2B }\end{array}$ \\
\hline GO:0031331 & $\begin{array}{l}\text { positive regulation of cellular catabolic } \\
\text { process }\end{array}$ & $9 / 117$ & $347 / 18493$ & 0.007 & $\begin{array}{c}\text { PSMC2 TFEB SNX9 GSK3A } \\
\text { GSK3B PABPC1 BAG6 CAV1 } \\
\text { SGTA }\end{array}$ \\
\hline GO: 1903050 & $\begin{array}{l}\text { regulation of proteolysis involved in } \\
\text { cellular protein catabolic process }\end{array}$ & $7 / 117$ & $209 / 18493$ & 0.007 & $\begin{array}{c}\text { PSMC2 GSK3A GSK3B RYBP } \\
\text { BAG6 CAV1 SGTA }\end{array}$ \\
\hline GO:0062014 & $\begin{array}{l}\text { negative regulation of small molecule } \\
\text { metabolic process }\end{array}$ & $5 / 117$ & $97 / 18493$ & 0.007 & $\begin{array}{c}\text { STAT3 TGFB1 CEACAM1 GSK3A } \\
\text { PARP1 }\end{array}$ \\
\hline
\end{tabular}




\begin{tabular}{|c|c|c|c|c|c|}
\hline ID & Description & GeneRatio & BgRatio & p.adjust & geneID \\
\hline GO:2000379 & $\begin{array}{l}\text { positive regulation of reactive oxygen } \\
\text { species metabolic process }\end{array}$ & $5 / 117$ & $98 / 18493$ & 0.007 & $\begin{array}{c}\text { TGFB1 SMAD3 COX2 ACE2 } \\
\text { DDAH2 }\end{array}$ \\
\hline GO:0030856 & regulation of epithelial cell differentiation & $6 / 117$ & $151 / 18493$ & 0.007 & $\begin{array}{c}\text { IKBKB CEACAM1 GSK3A GSK3B } \\
\text { H2AFY2 CAV1 }\end{array}$ \\
\hline GO:0060148 & $\begin{array}{l}\text { positive regulation of posttranscriptional } \\
\text { gene silencing }\end{array}$ & $3 / 117$ & $23 / 18493$ & 0.007 & STAT3 TGFB1 DDX5 \\
\hline GO:0017038 & protein import & $7 / 117$ & $214 / 18493$ & 0.007 & $\begin{array}{l}\text { KPNB1 STAT3 TGFB1 SMAD3 } \\
\text { KPNA2 KPNA4 HSPD1 }\end{array}$ \\
\hline GO:0018208 & peptidyl-proline modification & $4 / 117$ & $56 / 18493$ & 0.007 & PPIA FKBP1A PPIG PPIH \\
\hline GO:0050819 & negative regulation of coagulation & $4 / 117$ & $56 / 18493$ & 0.007 & $\begin{array}{l}\text { CD9 CEACAM1 ANXA2 } \\
\text { SERPING1 }\end{array}$ \\
\hline GO:0048732 & gland development & $10 / 117$ & $434 / 18493$ & 0.007 & $\begin{array}{c}\text { STAT5A JUN TGFB1 SMAD3 } \\
\text { COX2 BCL2 CEACAM1 ACO2 } \\
\text { RPL19 CAV1 }\end{array}$ \\
\hline GO:1901653 & cellular response to peptide & $9 / 117$ & $356 / 18493$ & 0.007 & $\begin{array}{c}\text { STAT5A STAT3 TGFB1 } \\
\text { CEACAM1 GSK3A GSK3B PARP1 } \\
\text { CAV1 ATP6V1G1 }\end{array}$ \\
\hline GO:1903203 & $\begin{array}{c}\text { regulation of oxidative stress-induced } \\
\text { neuron death }\end{array}$ & $3 / 117$ & $24 / 18493$ & 0.008 & MCL1 NONO PARP1 \\
\hline GO:0045216 & cell-cell junction organization & $6 / 117$ & $155 / 18493$ & 0.008 & $\begin{array}{c}\text { TGFB1 SMAD3 ACE2 CD9 IKBKB } \\
\text { CAV1 }\end{array}$ \\
\hline GO:0043393 & regulation of protein binding & $7 / 117$ & $217 / 18493$ & 0.008 & $\begin{array}{l}\text { PPP1CA BCL2 ANXA2 GSK3B } \\
\text { FKBP1A MARK3 CAV1 }\end{array}$ \\
\hline GO: 1902110 & $\begin{array}{l}\text { positive regulation of mitochondrial } \\
\text { membrane permeability involved in } \\
\text { apoptotic process }\end{array}$ & $4 / 117$ & $57 / 18493$ & 0.008 & BCL2 NMT1 GSK3A GSK3B \\
\hline GO:0006606 & protein import into nucleus & $6 / 117$ & $157 / 18493$ & 0.008 & $\begin{array}{c}\text { KPNB1 STAT3 TGFB1 SMAD3 } \\
\text { KPNA2 KPNA4 }\end{array}$ \\
\hline GO:2000378 & $\begin{array}{l}\text { negative regulation of reactive oxygen } \\
\text { species metabolic process }\end{array}$ & $4 / 117$ & $58 / 18493$ & 0.008 & STAT3 BCL2 HSPD1 CAV1 \\
\hline GO:0036475 & $\begin{array}{c}\text { neuron death in response to oxidative } \\
\text { stress }\end{array}$ & $3 / 117$ & $25 / 18493$ & 0.008 & MCL1 NONO PARP1 \\
\hline GO:0045088 & regulation of innate immune response & $9 / 117$ & $365 / 18493$ & 0.009 & $\begin{array}{l}\text { CD209 IRF3 SFTPD IKBKB } \\
\text { CEACAM1 HSPD1 NONO } \\
\text { SERPING1 CAV1 }\end{array}$ \\
\hline GO: 1902686 & $\begin{array}{l}\text { mitochondrial outer membrane } \\
\text { permeabilization involved in programmed } \\
\text { cell death }\end{array}$ & $4 / 117$ & $59 / 18493$ & 0.009 & BCL2 NMT1 GSK3A GSK3B \\
\hline GO:0051225 & spindle assembly & $5 / 117$ & $105 / 18493$ & 0.009 & $\begin{array}{c}\text { KPNB1 CHMP4B KIF11 CHMP2B } \\
\text { CHEK2 }\end{array}$ \\
\hline GO:1903037 & regulation of leukocyte cell-cell adhesion & $8 / 117$ & $293 / 18493$ & 0.009 & $\begin{array}{l}\text { TGFB1 CLEC4G CD209 SFTPD } \\
\text { DPP4 CEACAM1 HSPD1 CAV1 }\end{array}$ \\
\hline GO:0061077 & chaperone-mediated protein folding & $4 / 117$ & $60 / 18493$ & 0.009 & DNAJB1 HSPA9 FKBP1A SGTA \\
\hline GO:0035794 & $\begin{array}{l}\text { positive regulation of mitochondrial } \\
\text { membrane permeability }\end{array}$ & $4 / 117$ & $61 / 18493$ & 0.01 & BCL2 NMT1 GSK3A GSK3B \\
\hline
\end{tabular}




\begin{tabular}{|c|c|c|c|c|c|}
\hline ID & Description & GeneRatio & BgRatio & p.adjust & geneID \\
\hline GO:1904356 & $\begin{array}{l}\text { regulation of telomere maintenance via } \\
\text { telomere lengthening }\end{array}$ & $4 / 117$ & $61 / 18493$ & 0.01 & $\begin{array}{l}\text { HNRNPA1 HNRNPA2B1 PARP1 } \\
\text { TERF1 }\end{array}$ \\
\hline
\end{tabular}




\section{HCOV-CLUSTER_SET}

\subsection{GO ENRICHMENT}

GO Enrichment Analysis after FDR control of a gene set. 


\section{MOLECULAR FUNCTION}
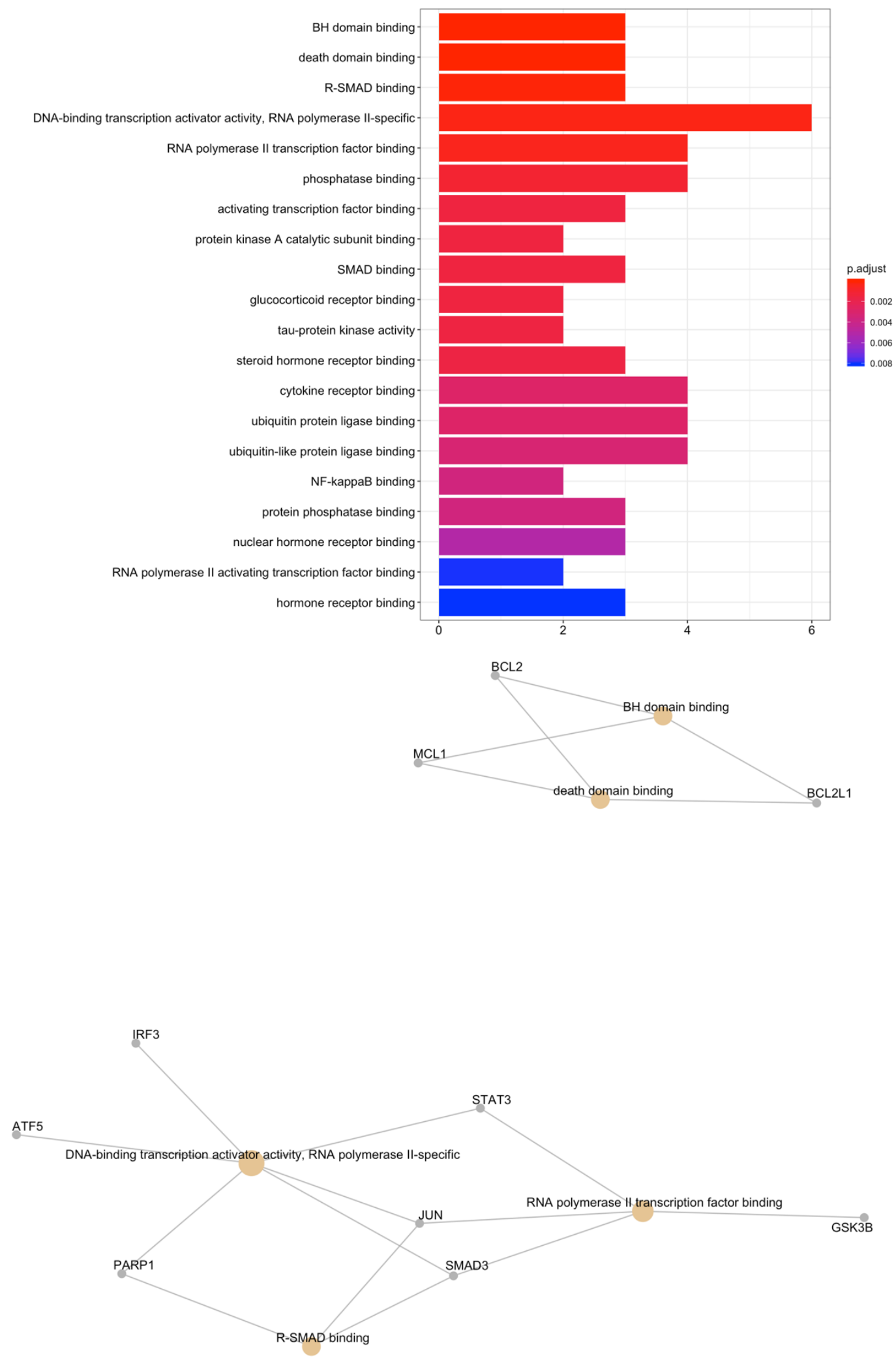


\section{CELLULAR COMPONENT}
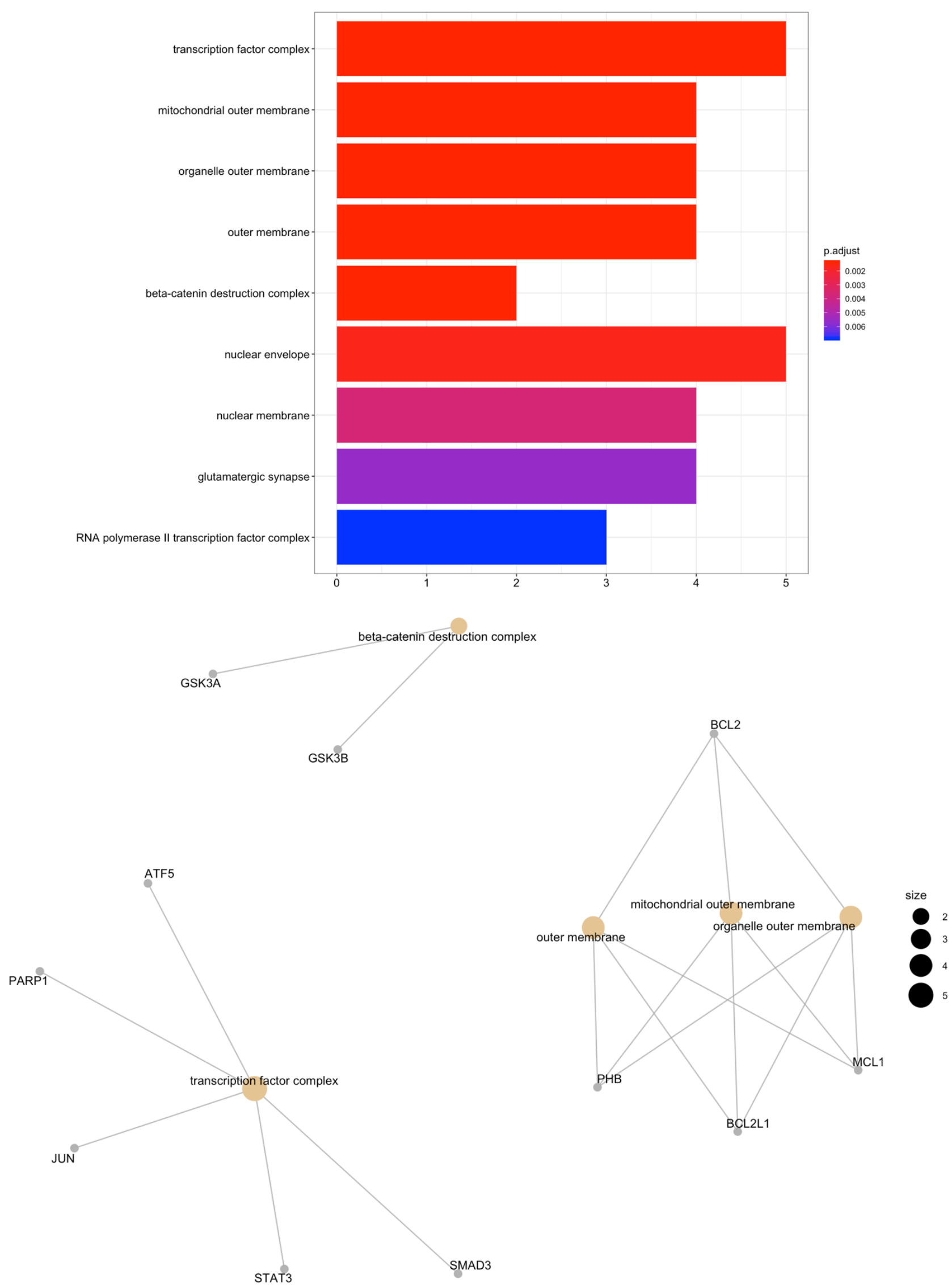


\section{BIOLOGICAL PROCESS}

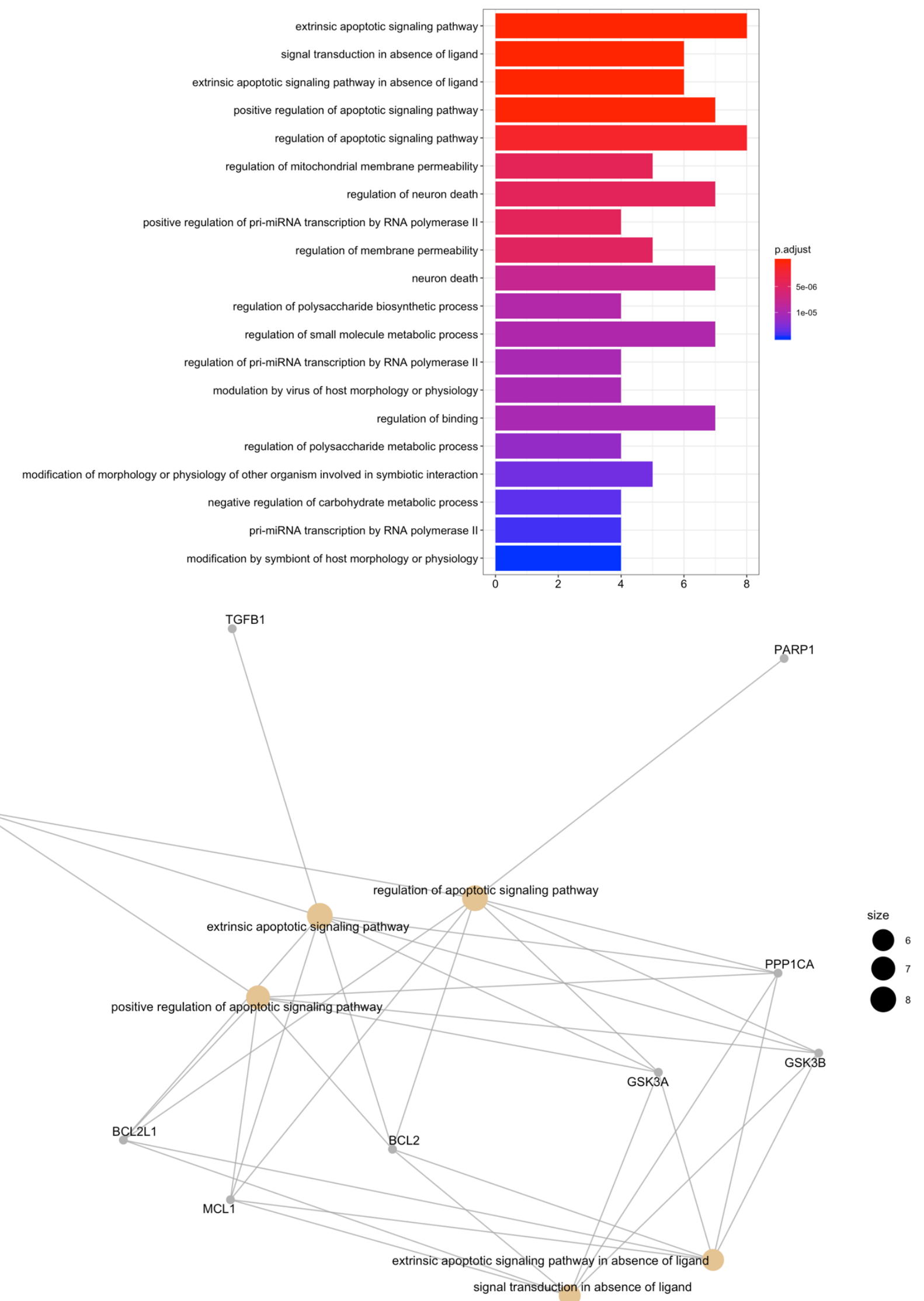




\subsubsection{MOLECULAR FUNCTION}

\begin{tabular}{|c|c|c|c|c|c|}
\hline ID & Description & GeneRatio & BgRatio & p.adjust & geneID \\
\hline GO:0051400 & BH domain binding & $3 / 21$ & $10 / 17632$ & 0 & BCL2L1 MCL1 BCL2 \\
\hline GO:0070513 & death domain binding & $3 / 21$ & $10 / 17632$ & 0 & BCL2L1 MCL1 BCL2 \\
\hline GO:0070412 & R-SMAD binding & $3 / 21$ & $23 / 17632$ & 0 & JUN SMAD3 PARP1 \\
\hline GO:0001228 & $\begin{array}{l}\text { DNA-binding transcription activator activity, RNA } \\
\text { polymerase II-specific }\end{array}$ & $6 / 21$ & $449 / 17632$ & 0 & $\begin{array}{l}\text { JUN STAT3 SMAD3 } \\
\text { ATF5 IRF3 PARP1 }\end{array}$ \\
\hline GO:0001085 & RNA polymerase II transcription factor binding & $4 / 21$ & $140 / 17632$ & 0.001 & $\begin{array}{l}\text { JUN STAT3 SMAD3 } \\
\text { GSK3B }\end{array}$ \\
\hline GO:0019902 & phosphatase binding & $4 / 21$ & $178 / 17632$ & 0.001 & $\begin{array}{l}\text { STAT3 PPP1CA } \\
\text { SMAD3 BCL2 }\end{array}$ \\
\hline GO:0033613 & activating transcription factor binding & $3 / 21$ & $76 / 17632$ & 0.001 & JUN SMAD3 NPM1 \\
\hline GO:0034236 & protein kinase A catalytic subunit binding & $2 / 21$ & $13 / 17632$ & 0.001 & GSK3A GSK3B \\
\hline GO:0046332 & SMAD binding & $3 / 21$ & $80 / 17632$ & 0.001 & JUN SMAD3 PARP1 \\
\hline GO:0035259 & glucocorticoid receptor binding & $2 / 21$ & $14 / 17632$ & 0.001 & STAT3 SMAD3 \\
\hline GO:0050321 & tau-protein kinase activity & $2 / 21$ & $15 / 17632$ & 0.002 & GSK3A GSK3B \\
\hline GO:0035258 & steroid hormone receptor binding & $3 / 21$ & $90 / 17632$ & 0.002 & $\begin{array}{l}\text { STAT3 SMAD3 } \\
\text { PARP1 }\end{array}$ \\
\hline GO:0005126 & cytokine receptor binding & $4 / 21$ & $281 / 17632$ & 0.003 & $\begin{array}{l}\text { STAT3 TGFB1 } \\
\text { SMAD3 IL6 }\end{array}$ \\
\hline GO:0031625 & ubiquitin protein ligase binding & $4 / 21$ & $286 / 17632$ & 0.003 & $\begin{array}{l}\text { JUN SMAD3 BCL2 } \\
\text { GSK3B }\end{array}$ \\
\hline GO:0044389 & ubiquitin-like protein ligase binding & $4 / 21$ & $301 / 17632$ & 0.003 & $\begin{array}{l}\text { JUN SMAD3 BCL2 } \\
\text { GSK3B }\end{array}$ \\
\hline GO:0051059 & NF-kappaB binding & $2 / 21$ & $28 / 17632$ & 0.004 & GSK3B NPM1 \\
\hline GO:0019903 & protein phosphatase binding & $3 / 21$ & $133 / 17632$ & 0.004 & STAT3 PPP1CA BCL2 \\
\hline GO:0035257 & nuclear hormone receptor binding & $3 / 21$ & $152 / 17632$ & 0.005 & $\begin{array}{l}\text { STAT3 SMAD3 } \\
\text { PARP1 }\end{array}$ \\
\hline GO:0001102 & $\begin{array}{l}\text { RNA polymerase II activating transcription factor } \\
\text { binding }\end{array}$ & $2 / 21$ & $44 / 17632$ & 0.008 & JUN SMAD3 \\
\hline GO:0051427 & hormone receptor binding & $3 / 21$ & $185 / 17632$ & 0.008 & $\begin{array}{l}\text { STAT3 SMAD3 } \\
\text { PARP1 }\end{array}$ \\
\hline GO:0051018 & protein kinase A binding & $2 / 21$ & $48 / 17632$ & 0.009 & GSK3A GSK3B \\
\hline GO:0005160 & transforming growth factor beta receptor binding & $2 / 21$ & $51 / 17632$ & 0.009 & TGFB1 SMAD3 \\
\hline
\end{tabular}




\subsubsection{CELLULAR COMPONENT}

\begin{tabular}{|c|c|c|c|c|c|}
\hline ID & Description & GeneRatio & BgRatio & p.adjust & geneID \\
\hline GO:0005667 & transcription factor complex & $5 / 21$ & $356 / 19659$ & 0.001 & $\begin{array}{c}\text { JUN STAT3 SMAD3 ATF5 } \\
\text { PARP1 }\end{array}$ \\
\hline GO:0005741 & mitochondrial outer membrane & $4 / 21$ & $180 / 19659$ & 0.001 & PHB BCL2L1 MCL1 BCL2 \\
\hline GO:0031968 & organelle outer membrane & $4 / 21$ & $204 / 19659$ & 0.001 & PHB BCL2L1 MCL1 BCL2 \\
\hline GO:0019867 & outer membrane & $4 / 21$ & $206 / 19659$ & 0.001 & PHB BCL2L1 MCL1 BCL2 \\
\hline GO:0030877 & beta-catenin destruction complex & $2 / 21$ & $12 / 19659$ & 0.001 & GSK3A GSK3B \\
\hline GO:0005635 & nuclear envelope & $5 / 21$ & $461 / 19659$ & 0.002 & $\begin{array}{l}\text { KPNB1 SMAD3 BCL2L1 } \\
\text { BCL2 PARP1 }\end{array}$ \\
\hline GO:0031965 & nuclear membrane & $4 / 21$ & $302 / 19659$ & 0.004 & $\begin{array}{l}\text { KPNB1 SMAD3 BCL2L1 } \\
\text { BCL2 }\end{array}$ \\
\hline GO:0098978 & glutamatergic synapse & $4 / 21$ & $350 / 19659$ & 0.006 & PHB STAT3 PPP1CA GSK3B \\
\hline GO:0090575 & $\begin{array}{l}\text { RNA polymerase II transcription factor } \\
\text { complex }\end{array}$ & $3 / 21$ & $161 / 19659$ & 0.007 & JUN STAT3 SMAD3 \\
\hline
\end{tabular}

\subsubsection{BIOLOGICAL PROCESS}

\begin{tabular}{|c|c|c|c|c|c|}
\hline ID & Description & GeneRatio & BgRatio & p.adjust & geneID \\
\hline GO:0097191 & extrinsic apoptotic signaling pathway & $8 / 20$ & $224 / 18493$ & 0 & $\begin{array}{c}\text { PPP1CA TGFB1 } \\
\text { SMAD3 BCL2L1 MCL1 } \\
\text { BCL2 GSK3A GSK3B }\end{array}$ \\
\hline GO:0038034 & signal transduction in absence of ligand & $6 / 20$ & $71 / 18493$ & 0 & $\begin{array}{l}\text { PPP1CA BCL2L1 MCL1 } \\
\text { BCL2 GSK3A GSK3B }\end{array}$ \\
\hline GO:0097192 & $\begin{array}{l}\text { extrinsic apoptotic signaling pathway in absence of } \\
\text { ligand }\end{array}$ & $6 / 20$ & $71 / 18493$ & 0 & $\begin{array}{l}\text { PPP1CA BCL2L1 MCL1 } \\
\text { BCL2 GSK3A GSK3B }\end{array}$ \\
\hline GO:2001235 & positive regulation of apoptotic signaling pathway & $7 / 20$ & $170 / 18493$ & 0 & $\begin{array}{l}\text { PPP1CA SMAD3 } \\
\text { BCL2L1 MCL1 BCL2 } \\
\text { GSK3A GSK3B }\end{array}$ \\
\hline GO:2001233 & regulation of apoptotic signaling pathway & $8 / 20$ & $391 / 18493$ & 0 & $\begin{array}{c}\text { PPP1CA SMAD3 } \\
\text { BCL2L1 MCL1 BCL2 } \\
\text { GSK3A GSK3B PARP1 }\end{array}$ \\
\hline GO:0046902 & regulation of mitochondrial membrane permeability & $5 / 20$ & $73 / 18493$ & 0 & $\begin{array}{l}\text { STAT3 BCL2L1 BCL2 } \\
\text { GSK3A GSK3B }\end{array}$ \\
\hline GO:1901214 & regulation of neuron death & $7 / 20$ & $296 / 18493$ & 0 & $\begin{array}{l}\text { JUN STAT3 BCL2L1 } \\
\text { MCL1 BCL2 GSK3B } \\
\text { PARP1 }\end{array}$ \\
\hline GO: 1902895 & $\begin{array}{l}\text { positive regulation of pri-miRNA transcription by } \\
\text { RNA polymerase II }\end{array}$ & $4 / 20$ & $27 / 18493$ & 0 & $\begin{array}{l}\text { JUN STAT3 TGFB1 } \\
\text { SMAD3 }\end{array}$ \\
\hline GO:0090559 & regulation of membrane permeability & $5 / 20$ & $81 / 18493$ & 0 & $\begin{array}{l}\text { STAT3 BCL2L1 BCL2 } \\
\text { GSK3A GSK3B }\end{array}$ \\
\hline
\end{tabular}




\begin{tabular}{|c|c|c|c|c|c|}
\hline ID & Description & GeneRatio & BgRatio & p.adjust & geneID \\
\hline GO:0070997 & neuron death & $7 / 20$ & $335 / 18493$ & 0 & $\begin{array}{l}\text { JUN STAT3 BCL2L1 } \\
\text { MCL1 BCL2 GSK3B } \\
\text { PARP1 }\end{array}$ \\
\hline GO:0032885 & regulation of polysaccharide biosynthetic process & $4 / 20$ & $35 / 18493$ & 0 & $\begin{array}{l}\text { PPP1CA TGFB1 GSK3A } \\
\text { GSK3B }\end{array}$ \\
\hline GO:0062012 & regulation of small molecule metabolic process & $7 / 20$ & $355 / 18493$ & 0 & $\begin{array}{c}\text { KPNB1 STAT3 PPP1CA } \\
\text { TGFB1 GSK3A GSK3B } \\
\text { PARP1 }\end{array}$ \\
\hline GO:1902893 & $\begin{array}{c}\text { regulation of pri-miRNA transcription by RNA } \\
\text { polymerase II }\end{array}$ & $4 / 20$ & $37 / 18493$ & 0 & $\begin{array}{l}\text { JUN STAT3 TGFB1 } \\
\text { SMAD3 }\end{array}$ \\
\hline GO:0019048 & modulation by virus of host morphology or physiology & $4 / 20$ & $38 / 18493$ & 0 & $\begin{array}{l}\text { KPNB1 TGFB1 SMAD3 } \\
\text { BCL2L1 }\end{array}$ \\
\hline GO:0051098 & regulation of binding & $7 / 20$ & $369 / 18493$ & 0 & $\begin{array}{c}\text { JUN PPP1CA TGFB1 } \\
\text { SMAD3 BCL2 GSK3B } \\
\text { PARP1 }\end{array}$ \\
\hline GO:0032881 & regulation of polysaccharide metabolic process & $4 / 20$ & $40 / 18493$ & 0 & $\begin{array}{l}\text { PPP1CA TGFB1 GSK3A } \\
\text { GSK3B }\end{array}$ \\
\hline GO:0051817 & $\begin{array}{l}\text { modification of morphology or physiology of other } \\
\text { organism involved in symbiotic interaction }\end{array}$ & $5 / 20$ & $112 / 18493$ & 0 & $\begin{array}{l}\text { KPNB1 JUN TGFB1 } \\
\text { SMAD3 BCL2L1 }\end{array}$ \\
\hline GO:0045912 & negative regulation of carbohydrate metabolic process & $4 / 20$ & $43 / 18493$ & 0 & $\begin{array}{l}\text { STAT3 TGFB1 GSK3A } \\
\text { GSK3B }\end{array}$ \\
\hline GO:0061614 & pri-miRNA transcription by RNA polymerase II & $4 / 20$ & $44 / 18493$ & 0 & $\begin{array}{l}\text { JUN STAT3 TGFB1 } \\
\text { SMAD3 }\end{array}$ \\
\hline GO:0044003 & $\begin{array}{l}\text { modification by symbiont of host morphology or } \\
\text { physiology }\end{array}$ & $4 / 20$ & $46 / 18493$ & 0 & $\begin{array}{l}\text { KPNB1 TGFB1 SMAD3 } \\
\text { BCL2L1 }\end{array}$ \\
\hline GO:2001239 & $\begin{array}{l}\text { regulation of extrinsic apoptotic signaling pathway in } \\
\text { absence of ligand }\end{array}$ & $4 / 20$ & $46 / 18493$ & 0 & $\begin{array}{l}\text { PPP1CA BCL2L1 MCL1 } \\
\text { BCL2 }\end{array}$ \\
\hline GO:0008637 & apoptotic mitochondrial changes & $5 / 20$ & $121 / 18493$ & 0 & $\begin{array}{l}\text { JUN BCL2L1 BCL2 } \\
\text { GSK3A GSK3B }\end{array}$ \\
\hline GO:1904951 & $\begin{array}{l}\text { positive regulation of establishment of protein } \\
\text { localization }\end{array}$ & $7 / 20$ & $437 / 18493$ & 0 & $\begin{array}{c}\text { TGFB1 SMAD3 IRF3 } \\
\text { BCL2 GSK3A GSK3B } \\
\text { IL6 }\end{array}$ \\
\hline GO:0007006 & mitochondrial membrane organization & $5 / 20$ & $131 / 18493$ & 0 & $\begin{array}{l}\text { STAT3 BCL2L1 BCL2 } \\
\text { GSK3A GSK3B }\end{array}$ \\
\hline GO:0009314 & response to radiation & $7 / 20$ & $443 / 18493$ & 0 & $\begin{array}{c}\text { JUN PPP1CA TGFB1 } \\
\text { BCL2L1 BCL2 NPM1 } \\
\text { PARP1 }\end{array}$ \\
\hline GO:0034504 & protein localization to nucleus & $6 / 20$ & $269 / 18493$ & 0 & $\begin{array}{l}\text { KPNB1 STAT3 TGFB1 } \\
\text { SMAD3 GSK3B PARP1 }\end{array}$ \\
\hline GO:0007183 & SMAD protein complex assembly & $3 / 20$ & $13 / 18493$ & 0 & TGFB1 SMAD3 PARP1 \\
\hline GO:0009895 & negative regulation of catabolic process & $6 / 20$ & $287 / 18493$ & 0 & $\begin{array}{l}\text { PHB STAT3 SMAD3 } \\
\text { MCL1 BCL2 GSK3A }\end{array}$ \\
\hline GO: 1901652 & response to peptide & $7 / 20$ & $481 / 18493$ & 0 & $\begin{array}{c}\text { STAT5A PHB STAT3 } \\
\text { TGFB1 GSK3A GSK3B } \\
\text { PARP1 }\end{array}$ \\
\hline GO:0035821 & $\begin{array}{c}\text { modification of morphology or physiology of other } \\
\text { organism }\end{array}$ & $5 / 20$ & $161 / 18493$ & 0 & $\begin{array}{l}\text { KPNB1 JUN TGFB1 } \\
\text { SMAD3 BCL2L1 }\end{array}$ \\
\hline
\end{tabular}




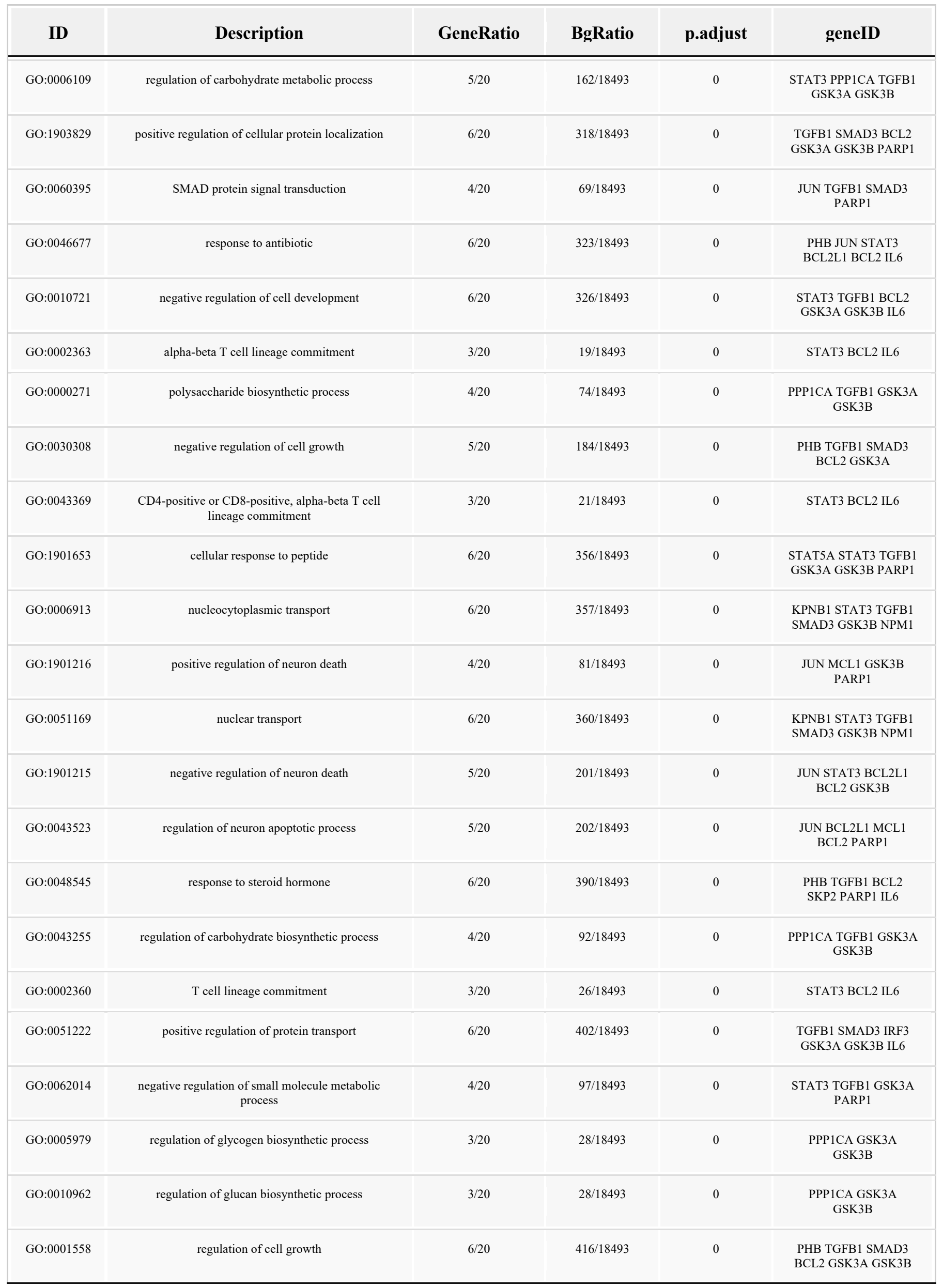




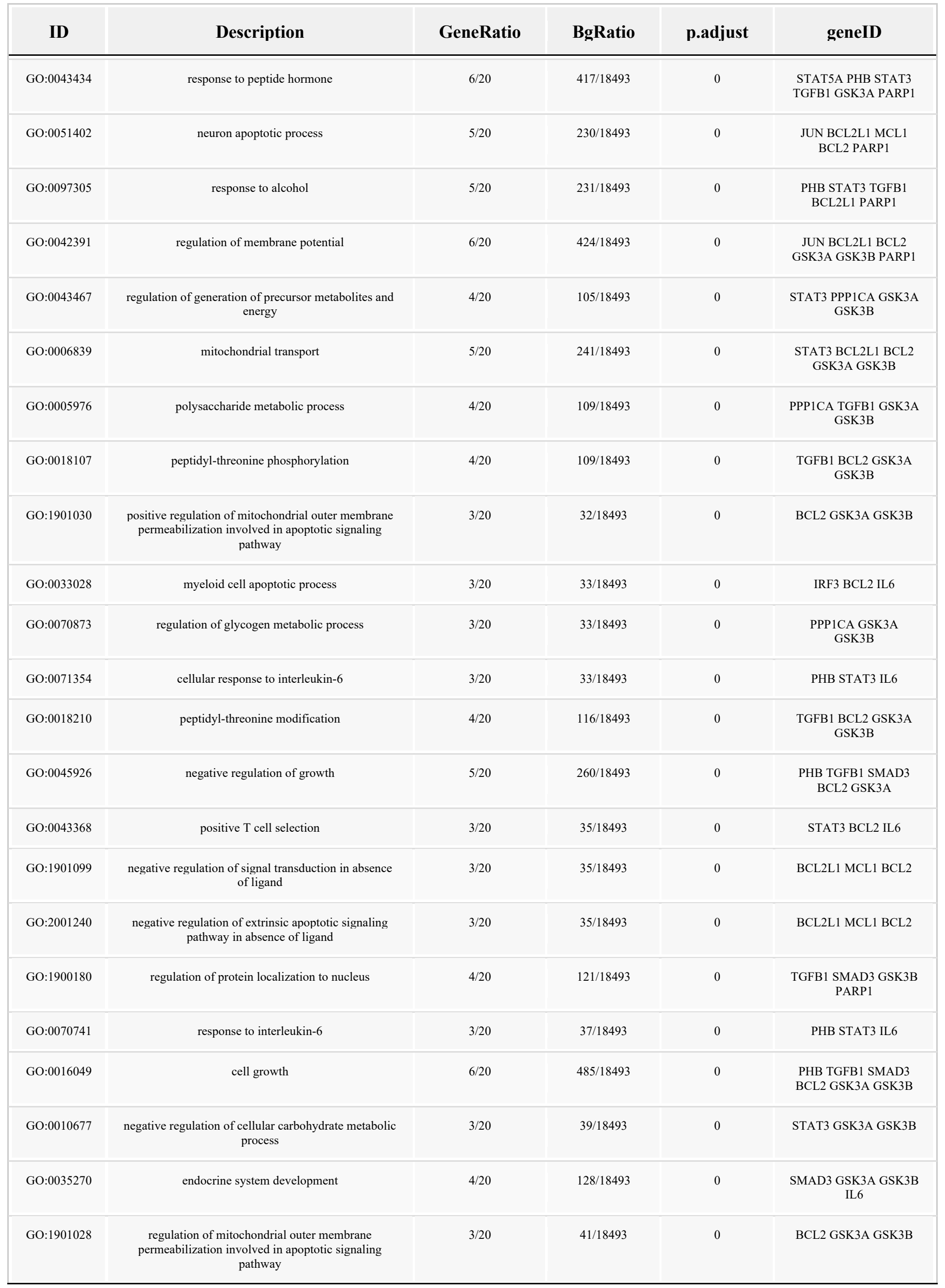




\begin{tabular}{|c|c|c|c|c|c|}
\hline ID & Description & GeneRatio & BgRatio & p.adjust & geneID \\
\hline GO:0030522 & intracellular receptor signaling pathway & $5 / 20$ & $288 / 18493$ & 0 & $\begin{array}{c}\text { PHB STAT3 IRF3 SKP2 } \\
\text { PARP1 }\end{array}$ \\
\hline GO:0034599 & cellular response to oxidative stress & $5 / 20$ & $289 / 18493$ & 0 & $\begin{array}{l}\text { JUN MCL1 BCL2 } \\
\text { PARP1 IL6 }\end{array}$ \\
\hline GO:0010675 & regulation of cellular carbohydrate metabolic process & $4 / 20$ & $134 / 18493$ & 0 & $\begin{array}{c}\text { STAT3 PPP1CA GSK3A } \\
\text { GSK3B }\end{array}$ \\
\hline GO:0018209 & peptidyl-serine modification & $5 / 20$ & $301 / 18493$ & 0 & $\begin{array}{l}\text { TGFB1 BCL2 GSK3B } \\
\text { PARP1 IL6 }\end{array}$ \\
\hline GO:0008631 & $\begin{array}{c}\text { intrinsic apoptotic signaling pathway in response to } \\
\text { oxidative stress }\end{array}$ & $3 / 20$ & $45 / 18493$ & 0 & MCL1 BCL2 PARP1 \\
\hline GO:0031018 & endocrine pancreas development & $3 / 20$ & $45 / 18493$ & 0 & GSK3A GSK3B IL6 \\
\hline GO:0071375 & cellular response to peptide hormone stimulus & $5 / 20$ & $307 / 18493$ & 0 & $\begin{array}{l}\text { STAT5A STAT3 TGFB1 } \\
\text { GSK3A PARP1 }\end{array}$ \\
\hline GO:0005978 & glycogen biosynthetic process & $3 / 20$ & $46 / 18493$ & 0 & $\begin{array}{l}\text { PPP1CA GSK3A } \\
\text { GSK3B }\end{array}$ \\
\hline GO:0009250 & glucan biosynthetic process & $3 / 20$ & $46 / 18493$ & 0 & $\begin{array}{c}\text { PPP1CA GSK3A } \\
\text { GSK3B }\end{array}$ \\
\hline GO:0010212 & response to ionizing radiation & $4 / 20$ & $146 / 18493$ & 0 & $\begin{array}{l}\text { TGFB1 BCL2L1 BCL2 } \\
\text { PARP1 }\end{array}$ \\
\hline GO:0007568 & aging & $5 / 20$ & $311 / 18493$ & 0 & $\begin{array}{l}\text { JUN STAT3 TGFB1 } \\
\text { BCL2 NPM1 }\end{array}$ \\
\hline GO:0035264 & multicellular organism growth & $4 / 20$ & $147 / 18493$ & 0 & $\begin{array}{l}\text { STAT5A STAT3 ATF5 } \\
\text { BCL2 }\end{array}$ \\
\hline GO:0009615 & response to virus & $5 / 20$ & $315 / 18493$ & 0 & $\begin{array}{l}\text { IRF3 BCL2L1 BCL2 } \\
\text { SKP2 IL6 }\end{array}$ \\
\hline GO:0016202 & regulation of striated muscle tissue development & $4 / 20$ & $149 / 18493$ & 0 & $\begin{array}{l}\text { TGFB1 SMAD3 BCL2 } \\
\text { GSK3A }\end{array}$ \\
\hline GO:0043470 & regulation of carbohydrate catabolic process & $3 / 20$ & $48 / 18493$ & 0 & STAT3 PPP1CA GSK3A \\
\hline GO:0045058 & $\mathrm{T}$ cell selection & $3 / 20$ & $48 / 18493$ & 0 & STAT3 BCL2 IL6 \\
\hline GO:1901861 & regulation of muscle tissue development & $4 / 20$ & $151 / 18493$ & 0 & $\begin{array}{l}\text { TGFB1 SMAD3 BCL2 } \\
\text { GSK3A }\end{array}$ \\
\hline GO:0045599 & negative regulation of fat cell differentiation & $3 / 20$ & $49 / 18493$ & 0 & TGFB1 SMAD3 IL6 \\
\hline GO:0048634 & regulation of muscle organ development & $4 / 20$ & $153 / 18493$ & 0 & $\begin{array}{c}\text { TGFB1 SMAD3 BCL2 } \\
\text { GSK3A }\end{array}$ \\
\hline GO:2001236 & regulation of extrinsic apoptotic signaling pathway & $4 / 20$ & $156 / 18493$ & 0 & $\begin{array}{l}\text { PPP1CA BCL2L1 MCL1 } \\
\text { BCL2 }\end{array}$ \\
\hline GO:0006606 & protein import into nucleus & $4 / 20$ & $157 / 18493$ & 0 & $\begin{array}{c}\text { KPNB1 STAT3 TGFB1 } \\
\text { SMAD3 }\end{array}$ \\
\hline GO:0097345 & mitochondrial outer membrane permeabilization & $3 / 20$ & $51 / 18493$ & 0 & BCL2 GSK3A GSK3B \\
\hline GO:0090316 & positive regulation of intracellular protein transport & $4 / 20$ & $158 / 18493$ & 0 & $\begin{array}{c}\text { TGFB1 SMAD3 GSK3A } \\
\text { GSK3B }\end{array}$ \\
\hline
\end{tabular}




\begin{tabular}{|c|c|c|c|c|c|}
\hline ID & Description & GeneRatio & BgRatio & p.adjust & geneID \\
\hline GO:0048638 & regulation of developmental growth & $5 / 20$ & $334 / 18493$ & 0 & $\begin{array}{l}\text { STAT5A STAT3 BCL2 } \\
\text { GSK3A GSK3B }\end{array}$ \\
\hline GO:2001242 & regulation of intrinsic apoptotic signaling pathway & $4 / 20$ & $161 / 18493$ & 0 & $\begin{array}{l}\text { BCL2L1 MCL1 BCL2 } \\
\text { PARP1 }\end{array}$ \\
\hline GO:0010332 & response to gamma radiation & $3 / 20$ & $53 / 18493$ & 0 & BCL2L1 BCL2 PARP1 \\
\hline GO:0031960 & response to corticosteroid & $4 / 20$ & $166 / 18493$ & 0.001 & $\begin{array}{l}\text { TGFB1 BCL2 PARP1 } \\
\text { IL6 }\end{array}$ \\
\hline GO:0001541 & ovarian follicle development & $3 / 20$ & $56 / 18493$ & 0.001 & PHB BCL2L1 BCL2 \\
\hline GO:0001654 & eye development & $5 / 20$ & $351 / 18493$ & 0.001 & $\begin{array}{l}\text { JUN STAT3 TGFB1 } \\
\text { SMAD3 BCL2 }\end{array}$ \\
\hline GO:0045862 & positive regulation of proteolysis & $5 / 20$ & $352 / 18493$ & 0.001 & $\begin{array}{l}\text { PHB STAT3 SMAD3 } \\
\text { GSK3A GSK3B }\end{array}$ \\
\hline GO:0150063 & visual system development & $5 / 20$ & $352 / 18493$ & 0.001 & $\begin{array}{l}\text { JUN STAT3 TGFB1 } \\
\text { SMAD3 BCL2 }\end{array}$ \\
\hline GO: 1902110 & $\begin{array}{l}\text { positive regulation of mitochondrial membrane } \\
\text { permeability involved in apoptotic process }\end{array}$ & $3 / 20$ & $57 / 18493$ & 0.001 & BCL2 GSK3A GSK3B \\
\hline GO:2001244 & $\begin{array}{l}\text { positive regulation of intrinsic apoptotic signaling } \\
\text { pathway }\end{array}$ & $3 / 20$ & $57 / 18493$ & 0.001 & BCL2L1 MCL1 BCL2 \\
\hline GO:0048880 & sensory system development & $5 / 20$ & $357 / 18493$ & 0.001 & $\begin{array}{l}\text { JUN STAT3 TGFB1 } \\
\text { SMAD3 BCL2 }\end{array}$ \\
\hline GO:0001836 & release of cytochrome $\mathrm{c}$ from mitochondria & $3 / 20$ & $58 / 18493$ & 0.001 & JUN BCL2L1 BCL2 \\
\hline GO:1902686 & $\begin{array}{l}\text { mitochondrial outer membrane permeabilization } \\
\text { involved in programmed cell death }\end{array}$ & $3 / 20$ & $59 / 18493$ & 0.001 & BCL2 GSK3A GSK3B \\
\hline GO:0010821 & regulation of mitochondrion organization & $4 / 20$ & $176 / 18493$ & 0.001 & $\begin{array}{l}\text { BCL2L1 BCL2 GSK3A } \\
\text { GSK3B }\end{array}$ \\
\hline GO:0051170 & import into nucleus & $4 / 20$ & $178 / 18493$ & 0.001 & $\begin{array}{l}\text { KPNB1 STAT3 TGFB1 } \\
\text { SMAD3 }\end{array}$ \\
\hline GO:0050678 & regulation of epithelial cell proliferation & $5 / 20$ & $368 / 18493$ & 0.001 & $\begin{array}{l}\text { STAT5A JUN STAT3 } \\
\text { TGFB1 SMAD3 }\end{array}$ \\
\hline GO:0035794 & $\begin{array}{l}\text { positive regulation of mitochondrial membrane } \\
\text { permeability }\end{array}$ & $3 / 20$ & $61 / 18493$ & 0.001 & BCL2 GSK3A GSK3B \\
\hline GO: 1902108 & $\begin{array}{l}\text { regulation of mitochondrial membrane permeability } \\
\text { involved in apoptotic process }\end{array}$ & $3 / 20$ & $63 / 18493$ & 0.001 & BCL2 GSK3A GSK3B \\
\hline GO: 1905710 & positive regulation of membrane permeability & $3 / 20$ & $63 / 18493$ & 0.001 & BCL2 GSK3A GSK3B \\
\hline GO:2000377 & $\begin{array}{l}\text { regulation of reactive oxygen species metabolic } \\
\text { process }\end{array}$ & $4 / 20$ & $185 / 18493$ & 0.001 & $\begin{array}{l}\text { STAT3 TGFB1 SMAD3 } \\
\text { BCL2 }\end{array}$ \\
\hline GO:0071478 & cellular response to radiation & $4 / 20$ & $186 / 18493$ & 0.001 & $\begin{array}{l}\text { TGFB1 BCL2L1 NPM1 } \\
\text { PARP1 }\end{array}$ \\
\hline GO:0001503 & ossification & $5 / 20$ & $380 / 18493$ & 0.001 & $\begin{array}{l}\text { PHB TGFB1 SMAD3 } \\
\text { BCL2 IL6 }\end{array}$ \\
\hline GO:0031099 & regeneration & $4 / 20$ & $188 / 18493$ & 0.001 & PHB JUN TGFB1 BCL2 \\
\hline
\end{tabular}




\begin{tabular}{|c|c|c|c|c|c|}
\hline ID & Description & GeneRatio & BgRatio & p.adjust & geneID \\
\hline GO:0007179 & $\begin{array}{l}\text { transforming growth factor beta receptor signaling } \\
\text { pathway }\end{array}$ & $4 / 20$ & $193 / 18493$ & 0.001 & $\begin{array}{l}\text { JUN TGFB1 SMAD3 } \\
\text { PARP1 }\end{array}$ \\
\hline GO:0007623 & circadian rhythm & $4 / 20$ & $193 / 18493$ & 0.001 & $\begin{array}{l}\text { JUN PPP1CA ATF5 } \\
\text { GSK3B }\end{array}$ \\
\hline GO:0030278 & regulation of ossification & $4 / 20$ & $193 / 18493$ & 0.001 & $\begin{array}{l}\text { TGFB1 SMAD3 BCL2 } \\
\text { IL6 }\end{array}$ \\
\hline GO:0046824 & positive regulation of nucleocytoplasmic transport & $3 / 20$ & $67 / 18493$ & 0.001 & TGFB1 SMAD3 GSK3B \\
\hline GO:0033148 & $\begin{array}{l}\text { positive regulation of intracellular estrogen receptor } \\
\text { signaling pathway }\end{array}$ & $2 / 20$ & $10 / 18493$ & 0.001 & SKP2 PARP1 \\
\hline GO:0071104 & response to interleukin-9 & $2 / 20$ & $10 / 18493$ & 0.001 & STAT5A STAT3 \\
\hline GO:0033692 & cellular polysaccharide biosynthetic process & $3 / 20$ & $68 / 18493$ & 0.001 & $\begin{array}{l}\text { PPP1CA GSK3A } \\
\text { GSK3B }\end{array}$ \\
\hline GO:0051881 & regulation of mitochondrial membrane potential & $3 / 20$ & $68 / 18493$ & 0.001 & BCL2L1 BCL2 PARP1 \\
\hline GO:0040014 & regulation of multicellular organism growth & $3 / 20$ & $69 / 18493$ & 0.001 & STAT5A STAT3 BCL2 \\
\hline GO:0050679 & positive regulation of epithelial cell proliferation & $4 / 20$ & $203 / 18493$ & 0.001 & $\begin{array}{l}\text { STAT5A JUN STAT3 } \\
\text { TGFB1 }\end{array}$ \\
\hline GO:1901201 & regulation of extracellular matrix assembly & $2 / 20$ & $11 / 18493$ & 0.001 & TGFB1 SMAD3 \\
\hline GO: 2001020 & regulation of response to DNA damage stimulus & $4 / 20$ & $206 / 18493$ & 0.001 & $\begin{array}{l}\text { BCL2L1 MCL1 BCL2 } \\
\text { PARP1 }\end{array}$ \\
\hline GO:0051090 & $\begin{array}{l}\text { regulation of DNA-binding transcription factor } \\
\text { activity }\end{array}$ & $5 / 20$ & $412 / 18493$ & 0.001 & $\begin{array}{l}\text { JUN TGFB1 SMAD3 } \\
\text { NPM1 IL6 }\end{array}$ \\
\hline GO:0005977 & glycogen metabolic process & $3 / 20$ & $73 / 18493$ & 0.001 & $\begin{array}{l}\text { PPP1CA GSK3A } \\
\text { GSK3B }\end{array}$ \\
\hline GO:0016051 & carbohydrate biosynthetic process & $4 / 20$ & $209 / 18493$ & 0.001 & $\begin{array}{l}\text { PPP1CA TGFB1 GSK3A } \\
\text { GSK3B }\end{array}$ \\
\hline GO:0051701 & interaction with host & $4 / 20$ & $209 / 18493$ & 0.001 & $\begin{array}{l}\text { KPNB1 TGFB1 SMAD3 } \\
\text { BCL2L1 }\end{array}$ \\
\hline GO:0006073 & cellular glucan metabolic process & $3 / 20$ & $74 / 18493$ & 0.001 & $\begin{array}{l}\text { PPP1CA GSK3A } \\
\text { GSK3B }\end{array}$ \\
\hline GO:0010611 & regulation of cardiac muscle hypertrophy & $3 / 20$ & $74 / 18493$ & 0.001 & SMAD3 GSK3A PARP1 \\
\hline GO:0044042 & glucan metabolic process & $3 / 20$ & $74 / 18493$ & 0.001 & $\begin{array}{l}\text { PPP1CA GSK3A } \\
\text { GSK3B }\end{array}$ \\
\hline GO:0048762 & mesenchymal cell differentiation & $4 / 20$ & $210 / 18493$ & 0.001 & $\begin{array}{l}\text { TGFB1 SMAD3 BCL2 } \\
\text { GSK3B }\end{array}$ \\
\hline GO:0050673 & epithelial cell proliferation & $5 / 20$ & $420 / 18493$ & 0.001 & $\begin{array}{l}\text { STAT5A JUN STAT3 } \\
\text { TGFB1 SMAD3 }\end{array}$ \\
\hline GO:0014743 & regulation of muscle hypertrophy & $3 / 20$ & $76 / 18493$ & 0.001 & SMAD3 GSK3A PARP1 \\
\hline GO:0060391 & $\begin{array}{l}\text { positive regulation of SMAD protein signal } \\
\text { transduction }\end{array}$ & $2 / 20$ & $12 / 18493$ & 0.001 & TGFB1 PARP1 \\
\hline GO:0072540 & T-helper 17 cell lineage commitment & $2 / 20$ & $12 / 18493$ & 0.001 & STAT3 IL6 \\
\hline
\end{tabular}




\begin{tabular}{|c|c|c|c|c|c|}
\hline ID & Description & GeneRatio & BgRatio & p.adjust & geneID \\
\hline GO:0017038 & protein import & $4 / 20$ & $214 / 18493$ & 0.001 & $\begin{array}{l}\text { KPNB1 STAT3 TGFB1 } \\
\text { SMAD3 }\end{array}$ \\
\hline GO:0032388 & positive regulation of intracellular transport & $4 / 20$ & $214 / 18493$ & 0.001 & $\begin{array}{c}\text { TGFB1 SMAD3 GSK3A } \\
\text { GSK3B }\end{array}$ \\
\hline GO:0031016 & pancreas development & $3 / 20$ & $77 / 18493$ & 0.001 & GSK3A GSK3B IL6 \\
\hline GO:0045444 & fat cell differentiation & $4 / 20$ & $217 / 18493$ & 0.001 & $\begin{array}{l}\text { TGFB1 SMAD3 ATF5 } \\
\text { IL6 }\end{array}$ \\
\hline GO: 1900182 & positive regulation of protein localization to nucleus & $3 / 20$ & $78 / 18493$ & 0.001 & TGFB1 SMAD3 PARP1 \\
\hline GO:0048732 & gland development & $5 / 20$ & $434 / 18493$ & 0.001 & $\begin{array}{l}\text { STAT5A JUN TGFB1 } \\
\text { SMAD3 BCL2 }\end{array}$ \\
\hline GO:0030279 & negative regulation of ossification & $3 / 20$ & $79 / 18493$ & 0.001 & TGFB1 SMAD3 BCL2 \\
\hline GO:0033143 & $\begin{array}{c}\text { regulation of intracellular steroid hormone receptor } \\
\text { signaling pathway }\end{array}$ & $3 / 20$ & $79 / 18493$ & 0.001 & PHB SKP2 PARP1 \\
\hline GO:0035723 & interleukin-15-mediated signaling pathway & $2 / 20$ & $13 / 18493$ & 0.001 & STAT5A STAT3 \\
\hline GO:0060397 & $\begin{array}{l}\text { JAK-STAT cascade involved in growth hormone } \\
\text { signaling pathway }\end{array}$ & $2 / 20$ & $13 / 18493$ & 0.001 & STAT5A STAT3 \\
\hline GO:0071350 & cellular response to interleukin- 15 & $2 / 20$ & $13 / 18493$ & 0.001 & STAT5A STAT3 \\
\hline GO:0006979 & response to oxidative stress & $5 / 20$ & $441 / 18493$ & 0.001 & $\begin{array}{l}\text { JUN MCL1 BCL2 } \\
\text { PARP1 IL6 }\end{array}$ \\
\hline GO:0051607 & defense response to virus & $4 / 20$ & $228 / 18493$ & 0.001 & IRF3 BCL2 SKP2 IL6 \\
\hline GO:0042326 & negative regulation of phosphorylation & $5 / 20$ & $448 / 18493$ & 0.001 & $\begin{array}{l}\text { PHB JUN STAT3 } \\
\text { TGFB1 NPM1 }\end{array}$ \\
\hline GO:0034637 & cellular carbohydrate biosynthetic process & $3 / 20$ & $83 / 18493$ & 0.001 & $\begin{array}{l}\text { PPP1CA GSK3A } \\
\text { GSK3B }\end{array}$ \\
\hline GO:0071216 & cellular response to biotic stimulus & $4 / 20$ & $229 / 18493$ & 0.001 & $\begin{array}{l}\text { TGFB1 IRF3 GSK3B } \\
\text { IL6 }\end{array}$ \\
\hline GO:0042110 & $\mathrm{T}$ cell activation & $5 / 20$ & $451 / 18493$ & 0.001 & $\begin{array}{l}\text { STAT3 TGFB1 SMAD3 } \\
\text { BCL2 IL6 }\end{array}$ \\
\hline GO:0045778 & positive regulation of ossification & $3 / 20$ & $84 / 18493$ & 0.001 & TGFB1 SMAD3 IL6 \\
\hline GO:0034248 & regulation of cellular amide metabolic process & $5 / 20$ & $453 / 18493$ & 0.001 & $\begin{array}{l}\text { STAT3 PPP1CA GSK3A } \\
\text { NPM1 IL6 }\end{array}$ \\
\hline GO:0033157 & regulation of intracellular protein transport & $4 / 20$ & $232 / 18493$ & 0.001 & $\begin{array}{c}\text { TGFB1 SMAD3 GSK3A } \\
\text { GSK3B }\end{array}$ \\
\hline GO:0043558 & $\begin{array}{l}\text { regulation of translational initiation in response to } \\
\text { stress }\end{array}$ & $2 / 20$ & $14 / 18493$ & 0.001 & PPP1CA NPM1 \\
\hline GO:0070672 & response to interleukin-15 & $2 / 20$ & $14 / 18493$ & 0.001 & STAT5A STAT3 \\
\hline GO:0030217 & $\mathrm{T}$ cell differentiation & $4 / 20$ & $234 / 18493$ & 0.001 & $\begin{array}{l}\text { STAT3 TGFB1 BCL2 } \\
\text { IL6 }\end{array}$ \\
\hline GO:0033002 & muscle cell proliferation & $4 / 20$ & $236 / 18493$ & 0.001 & JUN STAT3 SKP2 IL6 \\
\hline
\end{tabular}




\begin{tabular}{|c|c|c|c|c|c|}
\hline ID & Description & GeneRatio & BgRatio & p.adjust & geneID \\
\hline GO:0060828 & regulation of canonical Wnt signaling pathway & $4 / 20$ & $236 / 18493$ & 0.001 & $\begin{array}{l}\text { PPP1CA SMAD3 } \\
\text { GSK3A GSK3B }\end{array}$ \\
\hline GO:0006112 & energy reserve metabolic process & $3 / 20$ & $87 / 18493$ & 0.001 & $\begin{array}{l}\text { PPP1CA GSK3A } \\
\text { GSK3B }\end{array}$ \\
\hline GO:0001776 & leukocyte homeostasis & $3 / 20$ & $88 / 18493$ & 0.001 & TGFB1 BCL2 IL6 \\
\hline GO:0033689 & negative regulation of osteoblast proliferation & $2 / 20$ & $15 / 18493$ & 0.001 & SMAD3 BCL2 \\
\hline GO:2001170 & negative regulation of ATP biosynthetic process & $2 / 20$ & $15 / 18493$ & 0.001 & STAT3 PARP1 \\
\hline GO:0071560 & $\begin{array}{c}\text { cellular response to transforming growth factor beta } \\
\text { stimulus }\end{array}$ & $4 / 20$ & $242 / 18493$ & 0.001 & $\begin{array}{l}\text { JUN TGFB1 SMAD3 } \\
\text { PARP1 }\end{array}$ \\
\hline GO:0051899 & membrane depolarization & $3 / 20$ & $90 / 18493$ & 0.001 & JUN BCL2 PARP1 \\
\hline GO:0031330 & negative regulation of cellular catabolic process & $4 / 20$ & $245 / 18493$ & 0.001 & $\begin{array}{c}\text { STAT3 MCL1 BCL2 } \\
\text { GSK3A }\end{array}$ \\
\hline GO:0016311 & dephosphorylation & $5 / 20$ & $475 / 18493$ & 0.001 & $\begin{array}{c}\text { PPP1CA TGFB1 } \\
\text { SMAD3 BCL2 GSK3B }\end{array}$ \\
\hline GO:0045639 & positive regulation of myeloid cell differentiation & $3 / 20$ & $91 / 18493$ & 0.001 & JUN STAT3 TGFB1 \\
\hline GO:0048872 & homeostasis of number of cells & $4 / 20$ & $248 / 18493$ & 0.001 & $\begin{array}{l}\text { STAT3 TGFB1 BCL2 } \\
\text { IL6 }\end{array}$ \\
\hline GO:0071559 & response to transforming growth factor beta & $4 / 20$ & $249 / 18493$ & 0.001 & $\begin{array}{l}\text { JUN TGFB1 SMAD3 } \\
\text { PARP1 }\end{array}$ \\
\hline GO:0036473 & cell death in response to oxidative stress & $3 / 20$ & $93 / 18493$ & 0.001 & MCL1 BCL2 PARP1 \\
\hline GO:0002295 & T-helper cell lineage commitment & $2 / 20$ & $16 / 18493$ & 0.001 & STAT3 IL6 \\
\hline GO:0003007 & heart morphogenesis & $4 / 20$ & $253 / 18493$ & 0.002 & $\begin{array}{l}\text { JUN TGFB1 SMAD3 } \\
\text { GSK3A }\end{array}$ \\
\hline GO:0051091 & $\begin{array}{l}\text { positive regulation of DNA-binding transcription } \\
\text { factor activity }\end{array}$ & $4 / 20$ & $255 / 18493$ & 0.002 & $\begin{array}{l}\text { TGFB1 SMAD3 NPM1 } \\
\text { IL6 }\end{array}$ \\
\hline GO:0001657 & ureteric bud development & $3 / 20$ & $96 / 18493$ & 0.002 & TGFB1 SMAD3 BCL2 \\
\hline GO:2001243 & $\begin{array}{c}\text { negative regulation of intrinsic apoptotic signaling } \\
\text { pathway }\end{array}$ & $3 / 20$ & $96 / 18493$ & 0.002 & BCL2L1 MCL1 BCL2 \\
\hline GO:0045165 & cell fate commitment & $4 / 20$ & $257 / 18493$ & 0.002 & STAT3 MCL1 BCL2 IL6 \\
\hline GO:0072163 & mesonephric epithelium development & $3 / 20$ & $97 / 18493$ & 0.002 & TGFB1 SMAD3 BCL2 \\
\hline GO:0072164 & mesonephric tubule development & $3 / 20$ & $97 / 18493$ & 0.002 & TGFB1 SMAD3 BCL2 \\
\hline GO:0030809 & negative regulation of nucleotide biosynthetic process & $2 / 20$ & $17 / 18493$ & 0.002 & STAT3 PARP1 \\
\hline GO:0033145 & $\begin{array}{l}\text { positive regulation of intracellular steroid hormone } \\
\text { receptor signaling pathway }\end{array}$ & $2 / 20$ & $17 / 18493$ & 0.002 & SKP2 PARP1 \\
\hline GO:0043373 & CD4-positive, alpha-beta T cell lineage commitment & $2 / 20$ & $17 / 18493$ & 0.002 & STAT3 IL6 \\
\hline GO:0070102 & interleukin-6-mediated signaling pathway & $2 / 20$ & $17 / 18493$ & 0.002 & STAT3 IL6 \\
\hline GO: 1900372 & $\begin{array}{l}\text { negative regulation of purine nucleotide biosynthetic } \\
\text { process }\end{array}$ & $2 / 20$ & $17 / 18493$ & 0.002 & STAT3 PARP1 \\
\hline
\end{tabular}




\begin{tabular}{|c|c|c|c|c|c|}
\hline ID & Description & GeneRatio & BgRatio & p.adjust & geneID \\
\hline GO:2000811 & negative regulation of anoikis & $2 / 20$ & $17 / 18493$ & 0.002 & MCL1 BCL2 \\
\hline GO:0031334 & positive regulation of protein complex assembly & $4 / 20$ & $259 / 18493$ & 0.002 & $\begin{array}{l}\text { JUN TGFB1 GSK3B } \\
\text { PARP1 }\end{array}$ \\
\hline GO:0071383 & cellular response to steroid hormone stimulus & $4 / 20$ & $259 / 18493$ & 0.002 & $\begin{array}{l}\text { PHB TGFB1 SKP2 } \\
\text { PARP1 }\end{array}$ \\
\hline GO:0008585 & female gonad development & $3 / 20$ & $98 / 18493$ & 0.002 & PHB BCL2L1 BCL2 \\
\hline GO:0048661 & positive regulation of smooth muscle cell proliferation & $3 / 20$ & $98 / 18493$ & 0.002 & JUN SKP2 IL6 \\
\hline GO:0044264 & cellular polysaccharide metabolic process & $3 / 20$ & $99 / 18493$ & 0.002 & $\begin{array}{l}\text { PPP1CA GSK3A } \\
\text { GSK3B }\end{array}$ \\
\hline GO:0034976 & response to endoplasmic reticulum stress & $4 / 20$ & $264 / 18493$ & 0.002 & $\begin{array}{l}\text { JUN BCL2 GSK3A } \\
\text { GSK3B }\end{array}$ \\
\hline GO:0043555 & regulation of translation in response to stress & $2 / 20$ & $18 / 18493$ & 0.002 & PPP1CA NPM1 \\
\hline GO:0001823 & mesonephros development & $3 / 20$ & $101 / 18493$ & 0.002 & TGFB1 SMAD3 BCL2 \\
\hline GO:0046632 & alpha-beta $\mathrm{T}$ cell differentiation & $3 / 20$ & $101 / 18493$ & 0.002 & STAT3 BCL2 IL6 \\
\hline GO:0008630 & $\begin{array}{l}\text { intrinsic apoptotic signaling pathway in response to } \\
\text { DNA damage }\end{array}$ & $3 / 20$ & $102 / 18493$ & 0.002 & BCL2L1 MCL1 BCL2 \\
\hline GO:0060485 & mesenchyme development & $4 / 20$ & $269 / 18493$ & 0.002 & $\begin{array}{l}\text { TGFB1 SMAD3 BCL2 } \\
\text { GSK3B }\end{array}$ \\
\hline GO:0033138 & positive regulation of peptidyl-serine phosphorylation & $3 / 20$ & $103 / 18493$ & 0.002 & TGFB1 BCL2 IL6 \\
\hline GO:0046545 & development of primary female sexual characteristics & $3 / 20$ & $103 / 18493$ & 0.002 & PHB BCL2L1 BCL2 \\
\hline GO:2001237 & $\begin{array}{l}\text { negative regulation of extrinsic apoptotic signaling } \\
\text { pathway }\end{array}$ & $3 / 20$ & $104 / 18493$ & 0.002 & BCL2L1 MCL1 BCL2 \\
\hline GO:0010523 & $\begin{array}{l}\text { negative regulation of calcium ion transport into } \\
\text { cytosol }\end{array}$ & $2 / 20$ & $19 / 18493$ & 0.002 & TGFB1 BCL2 \\
\hline GO:0043502 & regulation of muscle adaptation & $3 / 20$ & $106 / 18493$ & 0.002 & SMAD3 GSK3A PARP1 \\
\hline GO:0048511 & rhythmic process & $4 / 20$ & $276 / 18493$ & 0.002 & $\begin{array}{l}\text { JUN PPP1CA ATF5 } \\
\text { GSK3B }\end{array}$ \\
\hline GO:0072593 & reactive oxygen species metabolic process & $4 / 20$ & $276 / 18493$ & 0.002 & $\begin{array}{l}\text { STAT3 TGFB1 SMAD3 } \\
\text { BCL2 }\end{array}$ \\
\hline GO:0003300 & cardiac muscle hypertrophy & $3 / 20$ & $107 / 18493$ & 0.002 & SMAD3 GSK3A PARP1 \\
\hline GO:0044262 & cellular carbohydrate metabolic process & $4 / 20$ & $278 / 18493$ & 0.002 & $\begin{array}{c}\text { STAT3 PPP1CA GSK3A } \\
\text { GSK3B }\end{array}$ \\
\hline GO:0050768 & negative regulation of neurogenesis & $4 / 20$ & $279 / 18493$ & 0.002 & $\begin{array}{c}\text { STAT3 TGFB1 GSK3B } \\
\text { IL6 }\end{array}$ \\
\hline GO:0045930 & negative regulation of mitotic cell cycle & $4 / 20$ & $281 / 18493$ & 0.002 & $\begin{array}{l}\text { TGFB1 SMAD3 } \\
\text { BCL2L1 BCL2 }\end{array}$ \\
\hline GO:0003323 & type B pancreatic cell development & $2 / 20$ & $20 / 18493$ & 0.002 & GSK3A GSK3B \\
\hline GO:0023019 & $\begin{array}{c}\text { signal transduction involved in regulation of gene } \\
\text { expression }\end{array}$ & $2 / 20$ & $20 / 18493$ & 0.002 & SMAD3 PARP1 \\
\hline
\end{tabular}




\begin{tabular}{|c|c|c|c|c|c|}
\hline ID & Description & GeneRatio & BgRatio & p.adjust & geneID \\
\hline GO:1903055 & positive regulation of extracellular matrix organization & $2 / 20$ & $20 / 18493$ & 0.002 & TGFB1 SMAD3 \\
\hline GO:0014897 & striated muscle hypertrophy & $3 / 20$ & $109 / 18493$ & 0.002 & SMAD3 GSK3A PARP1 \\
\hline GO:0018105 & peptidyl-serine phosphorylation & $4 / 20$ & $282 / 18493$ & 0.002 & $\begin{array}{l}\text { TGFB1 BCL2 GSK3B } \\
\text { IL6 }\end{array}$ \\
\hline GO:0060070 & canonical Wnt signaling pathway & $4 / 20$ & $284 / 18493$ & 0.002 & $\begin{array}{l}\text { PPP1CA SMAD3 } \\
\text { GSK3A GSK3B }\end{array}$ \\
\hline GO:0010906 & regulation of glucose metabolic process & $3 / 20$ & $111 / 18493$ & 0.002 & $\begin{array}{l}\text { PPP1CA GSK3A } \\
\text { GSK3B }\end{array}$ \\
\hline GO:0014896 & muscle hypertrophy & $3 / 20$ & $111 / 18493$ & 0.002 & SMAD3 GSK3A PARP1 \\
\hline GO: 1903828 & negative regulation of cellular protein localization & $3 / 20$ & $111 / 18493$ & 0.002 & TGFB1 BCL2L1 GSK3B \\
\hline GO:0097193 & intrinsic apoptotic signaling pathway & $4 / 20$ & $287 / 18493$ & 0.002 & $\begin{array}{l}\text { BCL2L1 MCL1 BCL2 } \\
\text { PARP1 }\end{array}$ \\
\hline GO:0001938 & positive regulation of endothelial cell proliferation & $3 / 20$ & $112 / 18493$ & 0.002 & STAT5A JUN STAT3 \\
\hline GO:0010822 & positive regulation of mitochondrion organization & $3 / 20$ & $112 / 18493$ & 0.002 & BCL2 GSK3A GSK3B \\
\hline GO:0046822 & regulation of nucleocytoplasmic transport & $3 / 20$ & $112 / 18493$ & 0.002 & TGFB1 SMAD3 GSK3B \\
\hline GO:0051900 & regulation of mitochondrial depolarization & $2 / 20$ & $21 / 18493$ & 0.002 & BCL2 PARP1 \\
\hline GO:0060390 & regulation of SMAD protein signal transduction & $2 / 20$ & $21 / 18493$ & 0.002 & TGFB1 PARP1 \\
\hline GO:0071359 & cellular response to dsRNA & $2 / 20$ & $21 / 18493$ & 0.002 & IRF3 NPM1 \\
\hline GO:1904886 & beta-catenin destruction complex disassembly & $2 / 20$ & $21 / 18493$ & 0.002 & PPP1CA GSK3B \\
\hline GO:0051153 & regulation of striated muscle cell differentiation & $3 / 20$ & $113 / 18493$ & 0.002 & TGFB1 BCL2 GSK3A \\
\hline GO:0006925 & inflammatory cell apoptotic process & $2 / 20$ & $22 / 18493$ & 0.002 & IRF3 IL6 \\
\hline GO: 1903077 & $\begin{array}{l}\text { negative regulation of protein localization to plasma } \\
\text { membrane }\end{array}$ & $2 / 20$ & $22 / 18493$ & 0.002 & TGFB1 BCL2L1 \\
\hline GO:2000637 & positive regulation of gene silencing by miRNA & $2 / 20$ & $22 / 18493$ & 0.002 & STAT3 TGFB1 \\
\hline GO:0006754 & ATP biosynthetic process & $3 / 20$ & $116 / 18493$ & 0.002 & STAT3 TGFB1 PARP1 \\
\hline GO:0001952 & regulation of cell-matrix adhesion & $3 / 20$ & $117 / 18493$ & 0.002 & SMAD3 BCL2 GSK3B \\
\hline GO:0051961 & negative regulation of nervous system development & $4 / 20$ & $298 / 18493$ & 0.002 & $\begin{array}{l}\text { STAT3 TGFB1 GSK3B } \\
\text { IL6 }\end{array}$ \\
\hline GO:0046660 & female sex differentiation & $3 / 20$ & $118 / 18493$ & 0.002 & PHB BCL2L1 BCL2 \\
\hline GO:0051882 & mitochondrial depolarization & $2 / 20$ & $23 / 18493$ & 0.002 & BCL2 PARP1 \\
\hline GO:0060148 & $\begin{array}{l}\text { positive regulation of posttranscriptional gene } \\
\text { silencing }\end{array}$ & $2 / 20$ & $23 / 18493$ & 0.002 & STAT3 TGFB1 \\
\hline GO:0060396 & growth hormone receptor signaling pathway & $2 / 20$ & $23 / 18493$ & 0.002 & STAT5A STAT3 \\
\hline GO:1903579 & negative regulation of ATP metabolic process & $2 / 20$ & $23 / 18493$ & 0.002 & STAT3 PARP1 \\
\hline
\end{tabular}




\begin{tabular}{|c|c|c|c|c|c|}
\hline ID & Description & GeneRatio & BgRatio & p.adjust & geneID \\
\hline GO:0010506 & regulation of autophagy & $4 / 20$ & $303 / 18493$ & 0.002 & $\begin{array}{l}\text { TFEB MCL1 BCL2 } \\
\text { GSK3A }\end{array}$ \\
\hline GO:0043500 & muscle adaptation & $3 / 20$ & $120 / 18493$ & 0.002 & SMAD3 GSK3A PARP1 \\
\hline GO:0051101 & regulation of DNA binding & $3 / 20$ & $122 / 18493$ & 0.002 & JUN TGFB1 PARP1 \\
\hline GO:0009416 & response to light stimulus & $4 / 20$ & $308 / 18493$ & 0.002 & $\begin{array}{l}\text { PPP1CA BCL2 NPM1 } \\
\text { PARP1 }\end{array}$ \\
\hline GO:0071378 & cellular response to growth hormone stimulus & $2 / 20$ & $24 / 18493$ & 0.002 & STAT5A STAT3 \\
\hline GO: 1903203 & regulation of oxidative stress-induced neuron death & $2 / 20$ & $24 / 18493$ & 0.002 & MCL1 PARP1 \\
\hline GO: 1904376 & $\begin{array}{l}\text { negative regulation of protein localization to cell } \\
\text { periphery }\end{array}$ & $2 / 20$ & $24 / 18493$ & 0.002 & TGFB1 BCL2L1 \\
\hline GO:2000209 & regulation of anoikis & $2 / 20$ & $24 / 18493$ & 0.002 & MCL1 BCL2 \\
\hline GO:2000679 & $\begin{array}{c}\text { positive regulation of transcription regulatory region } \\
\text { DNA binding }\end{array}$ & $2 / 20$ & $24 / 18493$ & 0.002 & TGFB1 PARP1 \\
\hline GO:0030111 & regulation of Wnt signaling pathway & $4 / 20$ & $311 / 18493$ & 0.002 & $\begin{array}{l}\text { PPP1CA SMAD3 } \\
\text { GSK3A GSK3B }\end{array}$ \\
\hline GO:0060562 & epithelial tube morphogenesis & $4 / 20$ & $313 / 18493$ & 0.003 & $\begin{array}{l}\text { PPP1CA TGFB1 } \\
\text { SMAD3 BCL2 }\end{array}$ \\
\hline GO:0002068 & glandular epithelial cell development & $2 / 20$ & $25 / 18493$ & 0.003 & GSK3A GSK3B \\
\hline GO:0003309 & type B pancreatic cell differentiation & $2 / 20$ & $25 / 18493$ & 0.003 & GSK3A GSK3B \\
\hline GO:0019054 & modulation by virus of host process & $2 / 20$ & $25 / 18493$ & 0.003 & KPNB1 BCL2L1 \\
\hline GO:0036475 & neuron death in response to oxidative stress & $2 / 20$ & $25 / 18493$ & 0.003 & MCL1 PARP1 \\
\hline GO:0085029 & extracellular matrix assembly & $2 / 20$ & $25 / 18493$ & 0.003 & TGFB1 SMAD3 \\
\hline GO: 1900543 & $\begin{array}{l}\text { negative regulation of purine nucleotide metabolic } \\
\text { process }\end{array}$ & $2 / 20$ & $25 / 18493$ & 0.003 & STAT3 PARP1 \\
\hline GO:0009206 & $\begin{array}{l}\text { purine ribonucleoside triphosphate biosynthetic } \\
\text { process }\end{array}$ & $3 / 20$ & $127 / 18493$ & 0.003 & STAT3 TGFB1 PARP1 \\
\hline GO:0006470 & protein dephosphorylation & $4 / 20$ & $318 / 18493$ & 0.003 & $\begin{array}{l}\text { PPP1CA TGFB1 BCL2 } \\
\text { GSK3B }\end{array}$ \\
\hline GO:0071214 & cellular response to abiotic stimulus & $4 / 20$ & $319 / 18493$ & 0.003 & $\begin{array}{l}\text { TGFB1 BCL2L1 NPM1 } \\
\text { PARP1 }\end{array}$ \\
\hline GO:0104004 & cellular response to environmental stimulus & $4 / 20$ & $319 / 18493$ & 0.003 & $\begin{array}{l}\text { TGFB1 BCL2L1 NPM1 } \\
\text { PARP1 }\end{array}$ \\
\hline GO:0009145 & purine nucleoside triphosphate biosynthetic process & $3 / 20$ & $128 / 18493$ & 0.003 & STAT3 TGFB1 PARP1 \\
\hline GO:0045598 & regulation of fat cell differentiation & $3 / 20$ & $128 / 18493$ & 0.003 & TGFB1 SMAD3 IL6 \\
\hline GO:0050921 & positive regulation of chemotaxis & $3 / 20$ & $130 / 18493$ & 0.003 & TGFB1 SMAD3 IL6 \\
\hline GO:0045980 & negative regulation of nucleotide metabolic process & $2 / 20$ & $26 / 18493$ & 0.003 & STAT3 PARP1 \\
\hline GO:0072539 & T-helper 17 cell differentiation & $2 / 20$ & $26 / 18493$ & 0.003 & STAT3 IL6 \\
\hline
\end{tabular}




\begin{tabular}{|c|c|c|c|c|c|}
\hline ID & Description & GeneRatio & BgRatio & p.adjust & geneID \\
\hline GO:0032496 & response to lipopolysaccharide & $4 / 20$ & $324 / 18493$ & 0.003 & JUN TGFB1 IRF3 IL6 \\
\hline GO:0048565 & digestive tract development & $3 / 20$ & $131 / 18493$ & 0.003 & TGFB1 SMAD3 BCL2 \\
\hline GO:0071496 & cellular response to external stimulus & $4 / 20$ & $328 / 18493$ & 0.003 & $\begin{array}{l}\text { JUN TGFB1 BCL2 } \\
\text { SKP2 }\end{array}$ \\
\hline GO:0009201 & ribonucleoside triphosphate biosynthetic process & $3 / 20$ & $133 / 18493$ & 0.003 & STAT3 TGFB1 PARP1 \\
\hline GO:0035883 & enteroendocrine cell differentiation & $2 / 20$ & $27 / 18493$ & 0.003 & GSK3A GSK3B \\
\hline GO:0035902 & response to immobilization stress & $2 / 20$ & $27 / 18493$ & 0.003 & PHB TGFB1 \\
\hline GO:0046631 & alpha-beta $\mathrm{T}$ cell activation & $3 / 20$ & $134 / 18493$ & 0.003 & STAT3 BCL2 IL6 \\
\hline GO:0001837 & epithelial to mesenchymal transition & $3 / 20$ & $135 / 18493$ & 0.003 & TGFB1 SMAD3 GSK3B \\
\hline GO:0033135 & regulation of peptidyl-serine phosphorylation & $3 / 20$ & $135 / 18493$ & 0.003 & TGFB1 BCL2 IL6 \\
\hline GO:0009411 & response to UV & $3 / 20$ & $136 / 18493$ & 0.003 & BCL2 NPM1 PARP1 \\
\hline GO:0033688 & regulation of osteoblast proliferation & $2 / 20$ & $28 / 18493$ & 0.003 & SMAD3 BCL2 \\
\hline GO:0072538 & T-helper 17 type immune response & $2 / 20$ & $28 / 18493$ & 0.003 & STAT3 IL6 \\
\hline GO:1904646 & cellular response to amyloid-beta & $2 / 20$ & $28 / 18493$ & 0.003 & GSK3B PARP1 \\
\hline GO:0001889 & liver development & $3 / 20$ & $137 / 18493$ & 0.003 & JUN TGFB1 SMAD3 \\
\hline GO:0002237 & response to molecule of bacterial origin & $4 / 20$ & $337 / 18493$ & 0.003 & JUN TGFB1 IRF3 IL6 \\
\hline GO:0007178 & $\begin{array}{l}\text { transmembrane receptor protein serine/threonine } \\
\text { kinase signaling pathway }\end{array}$ & $4 / 20$ & $340 / 18493$ & 0.003 & $\begin{array}{l}\text { JUN TGFB1 SMAD3 } \\
\text { PARP1 }\end{array}$ \\
\hline GO:0034250 & positive regulation of cellular amide metabolic process & $3 / 20$ & $140 / 18493$ & 0.003 & GSK3A NPM1 IL6 \\
\hline GO:0061008 & hepaticobiliary system development & $3 / 20$ & $140 / 18493$ & 0.003 & JUN TGFB1 SMAD3 \\
\hline GO:0072073 & kidney epithelium development & $3 / 20$ & $140 / 18493$ & 0.003 & TGFB1 SMAD3 BCL2 \\
\hline GO:0044068 & modulation by symbiont of host cellular process & $2 / 20$ & $29 / 18493$ & 0.003 & KPNB1 BCL2L1 \\
\hline GO:0070306 & lens fiber cell differentiation & $2 / 20$ & $29 / 18493$ & 0.003 & TGFB1 SMAD3 \\
\hline GO: 1902175 & $\begin{array}{l}\text { regulation of oxidative stress-induced intrinsic } \\
\text { apoptotic signaling pathway }\end{array}$ & $2 / 20$ & $29 / 18493$ & 0.003 & MCL1 PARP1 \\
\hline GO:0009127 & purine nucleoside monophosphate biosynthetic process & $3 / 20$ & $141 / 18493$ & 0.003 & STAT3 TGFB1 PARP1 \\
\hline GO:0009168 & $\begin{array}{l}\text { purine ribonucleoside monophosphate biosynthetic } \\
\text { process }\end{array}$ & $3 / 20$ & $141 / 18493$ & 0.003 & STAT3 TGFB1 PARP1 \\
\hline GO:0042542 & response to hydrogen peroxide & $3 / 20$ & $141 / 18493$ & 0.003 & JUN BCL2 IL6 \\
\hline GO:0030098 & lymphocyte differentiation & $4 / 20$ & $344 / 18493$ & 0.003 & $\begin{array}{l}\text { STAT3 TGFB1 BCL2 } \\
\text { IL6 }\end{array}$ \\
\hline GO:0030518 & $\begin{array}{l}\text { intracellular steroid hormone receptor signaling } \\
\text { pathway }\end{array}$ & $3 / 20$ & $142 / 18493$ & 0.003 & PHB SKP2 PARP1 \\
\hline GO:0055123 & digestive system development & $3 / 20$ & $143 / 18493$ & 0.003 & TGFB1 SMAD3 BCL2 \\
\hline
\end{tabular}




\begin{tabular}{|c|c|c|c|c|c|}
\hline ID & Description & GeneRatio & BgRatio & p.adjust & geneID \\
\hline GO:0038111 & interleukin-7-mediated signaling pathway & $2 / 20$ & $30 / 18493$ & 0.003 & STAT5A STAT3 \\
\hline GO: 1905476 & $\begin{array}{l}\text { negative regulation of protein localization to } \\
\text { membrane }\end{array}$ & $2 / 20$ & $30 / 18493$ & 0.003 & TGFB1 BCL2L1 \\
\hline GO:0006959 & humoral immune response & $4 / 20$ & $349 / 18493$ & 0.003 & TFEB PHB BCL2 IL6 \\
\hline GO:0050871 & positive regulation of $\mathrm{B}$ cell activation & $3 / 20$ & $144 / 18493$ & 0.003 & TGFB1 BCL2 IL6 \\
\hline GO:0009142 & nucleoside triphosphate biosynthetic process & $3 / 20$ & $145 / 18493$ & 0.003 & STAT3 TGFB1 PARP1 \\
\hline GO:0033687 & osteoblast proliferation & $2 / 20$ & $31 / 18493$ & 0.003 & SMAD3 BCL2 \\
\hline GO:0045745 & $\begin{array}{c}\text { positive regulation of G protein-coupled receptor } \\
\text { signaling pathway }\end{array}$ & $2 / 20$ & $31 / 18493$ & 0.003 & PHB GSK3A \\
\hline GO:0098760 & response to interleukin-7 & $2 / 20$ & $31 / 18493$ & 0.003 & STAT5A STAT3 \\
\hline GO:0098761 & cellular response to interleukin-7 & $2 / 20$ & $31 / 18493$ & 0.003 & STAT5A STAT3 \\
\hline GO: 1902230 & $\begin{array}{l}\text { negative regulation of intrinsic apoptotic signaling } \\
\text { pathway in response to DNA damage }\end{array}$ & $2 / 20$ & $31 / 18493$ & 0.003 & BCL2L1 BCL2 \\
\hline GO: 1904645 & response to amyloid-beta & $2 / 20$ & $31 / 18493$ & 0.003 & GSK3B PARP1 \\
\hline GO:0007259 & JAK-STAT cascade & $3 / 20$ & $147 / 18493$ & 0.003 & STAT5A STAT3 IL6 \\
\hline GO:0043524 & negative regulation of neuron apoptotic process & $3 / 20$ & $147 / 18493$ & 0.003 & JUN BCL2L1 BCL2 \\
\hline GO:0010038 & response to metal ion & $4 / 20$ & $358 / 18493$ & 0.003 & $\begin{array}{l}\text { JUN PPP1CA BCL2 } \\
\text { PARP1 }\end{array}$ \\
\hline GO:0051384 & response to glucocorticoid & $3 / 20$ & $149 / 18493$ & 0.003 & TGFB1 BCL2 IL6 \\
\hline GO:0048754 & branching morphogenesis of an epithelial tube & $3 / 20$ & $150 / 18493$ & 0.004 & PPP1CA TGFB1 BCL2 \\
\hline GO:0010574 & $\begin{array}{l}\text { regulation of vascular endothelial growth factor } \\
\text { production }\end{array}$ & $2 / 20$ & $32 / 18493$ & 0.004 & TGFB1 IL6 \\
\hline GO:0097696 & STAT cascade & $3 / 20$ & $151 / 18493$ & 0.004 & STAT5A STAT3 IL6 \\
\hline GO:0016052 & carbohydrate catabolic process & $3 / 20$ & $152 / 18493$ & 0.004 & STAT3 PPP1CA GSK3A \\
\hline GO:0009156 & ribonucleoside monophosphate biosynthetic process & $3 / 20$ & $155 / 18493$ & 0.004 & STAT3 TGFB1 PARP1 \\
\hline GO:0010573 & vascular endothelial growth factor production & $2 / 20$ & $34 / 18493$ & 0.004 & TGFB1 IL6 \\
\hline GO:0043276 & anoikis & $2 / 20$ & $34 / 18493$ & 0.004 & MCL1 BCL2 \\
\hline GO:0042176 & regulation of protein catabolic process & $4 / 20$ & $375 / 18493$ & 0.004 & $\begin{array}{l}\text { PHB SMAD3 GSK3A } \\
\text { GSK3B }\end{array}$ \\
\hline GO:0010614 & negative regulation of cardiac muscle hypertrophy & $2 / 20$ & $35 / 18493$ & 0.004 & SMAD3 GSK3A \\
\hline GO:0030501 & positive regulation of bone mineralization & $2 / 20$ & $35 / 18493$ & 0.004 & TGFB1 SMAD3 \\
\hline GO:0014706 & striated muscle tissue development & $4 / 20$ & $379 / 18493$ & 0.004 & $\begin{array}{l}\text { TGFB1 SMAD3 BCL2 } \\
\text { GSK3A }\end{array}$ \\
\hline GO:0060416 & response to growth hormone & $2 / 20$ & $36 / 18493$ & 0.004 & STAT5A STAT3 \\
\hline
\end{tabular}




\begin{tabular}{|c|c|c|c|c|c|}
\hline ID & Description & GeneRatio & BgRatio & p.adjust & geneID \\
\hline GO:0018108 & peptidyl-tyrosine phosphorylation & $4 / 20$ & $387 / 18493$ & 0.004 & $\begin{array}{l}\text { STAT5A STAT3 TGFB1 } \\
\text { IL6 }\end{array}$ \\
\hline GO:0010634 & positive regulation of epithelial cell migration & $3 / 20$ & $165 / 18493$ & 0.004 & STAT5A JUN TGFB1 \\
\hline GO:0051100 & negative regulation of binding & $3 / 20$ & $165 / 18493$ & 0.004 & JUN PPP1CA GSK3B \\
\hline GO:0018212 & peptidyl-tyrosine modification & $4 / 20$ & $390 / 18493$ & 0.004 & $\begin{array}{l}\text { STAT5A STAT3 TGFB1 } \\
\text { IL6 }\end{array}$ \\
\hline GO:0014741 & negative regulation of muscle hypertrophy & $2 / 20$ & $37 / 18493$ & 0.004 & SMAD3 GSK3A \\
\hline GO:0009124 & nucleoside monophosphate biosynthetic process & $3 / 20$ & $167 / 18493$ & 0.005 & STAT3 TGFB1 PARP1 \\
\hline GO:0048660 & regulation of smooth muscle cell proliferation & $3 / 20$ & $168 / 18493$ & 0.005 & JUN SKP2 IL6 \\
\hline GO:0060537 & muscle tissue development & $4 / 20$ & $395 / 18493$ & 0.005 & $\begin{array}{l}\text { TGFB1 SMAD3 BCL2 } \\
\text { GSK3A }\end{array}$ \\
\hline GO:0000060 & protein import into nucleus, translocation & $2 / 20$ & $38 / 18493$ & 0.005 & KPNB1 TGFB1 \\
\hline GO:0045785 & positive regulation of cell adhesion & $4 / 20$ & $397 / 18493$ & 0.005 & $\begin{array}{l}\text { TGFB1 SMAD3 GSK3B } \\
\text { IL6 }\end{array}$ \\
\hline GO:0048659 & smooth muscle cell proliferation & $3 / 20$ & $170 / 18493$ & 0.005 & JUN SKP2 IL6 \\
\hline GO:0033146 & $\begin{array}{l}\text { regulation of intracellular estrogen receptor signaling } \\
\text { pathway }\end{array}$ & $2 / 20$ & $39 / 18493$ & 0.005 & SKP2 PARP1 \\
\hline GO:0043029 & $\mathrm{T}$ cell homeostasis & $2 / 20$ & $39 / 18493$ & 0.005 & TGFB1 BCL2 \\
\hline GO: 1902229 & $\begin{array}{l}\text { regulation of intrinsic apoptotic signaling pathway in } \\
\text { response to DNA damage }\end{array}$ & $2 / 20$ & $39 / 18493$ & 0.005 & BCL2L1 BCL2 \\
\hline GO: 1903053 & regulation of extracellular matrix organization & $2 / 20$ & $39 / 18493$ & 0.005 & TGFB1 SMAD3 \\
\hline GO:0006417 & regulation of translation & $4 / 20$ & $402 / 18493$ & 0.005 & $\begin{array}{l}\text { STAT3 PPP1CA NPM1 } \\
\text { IL6 }\end{array}$ \\
\hline GO:0007517 & muscle organ development & $4 / 20$ & $403 / 18493$ & 0.005 & $\begin{array}{l}\text { TGFB1 SMAD3 BCL2 } \\
\text { GSK3A }\end{array}$ \\
\hline GO:0002285 & lymphocyte activation involved in immune response & $3 / 20$ & $174 / 18493$ & 0.005 & STAT3 TGFB1 IL6 \\
\hline GO:0030099 & myeloid cell differentiation & $4 / 20$ & $405 / 18493$ & 0.005 & $\begin{array}{l}\text { JUN STAT3 TGFB1 } \\
\text { PARP1 }\end{array}$ \\
\hline GO:0001936 & regulation of endothelial cell proliferation & $3 / 20$ & $175 / 18493$ & 0.005 & STAT5A JUN STAT3 \\
\hline GO:0070507 & regulation of microtubule cytoskeleton organization & $3 / 20$ & $175 / 18493$ & 0.005 & ATF5 GSK3B NPM1 \\
\hline GO:0030225 & macrophage differentiation & $2 / 20$ & $40 / 18493$ & 0.005 & TGFB1 PARP1 \\
\hline GO:0044275 & cellular carbohydrate catabolic process & $2 / 20$ & $40 / 18493$ & 0.005 & PPP1CA GSK3A \\
\hline GO:0001933 & negative regulation of protein phosphorylation & $4 / 20$ & $408 / 18493$ & 0.005 & PHB JUN TGFB1 NPM1 \\
\hline GO: 1905475 & regulation of protein localization to membrane & $3 / 20$ & $177 / 18493$ & 0.005 & TGFB1 BCL2L1 BCL2 \\
\hline GO:0051052 & regulation of DNA metabolic process & $4 / 20$ & $412 / 18493$ & 0.005 & $\begin{array}{l}\text { JUN TGFB1 NPM1 } \\
\text { PARP1 }\end{array}$ \\
\hline
\end{tabular}




\begin{tabular}{|c|c|c|c|c|c|}
\hline ID & Description & GeneRatio & BgRatio & p.adjust & geneID \\
\hline GO:1901987 & regulation of cell cycle phase transition & $4 / 20$ & $412 / 18493$ & 0.005 & $\begin{array}{l}\text { TGFB1 ATF5 BCL2 } \\
\text { NPM1 }\end{array}$ \\
\hline GO:0006611 & protein export from nucleus & $3 / 20$ & $180 / 18493$ & 0.005 & TGFB1 GSK3B NPM1 \\
\hline GO:0051099 & positive regulation of binding & $3 / 20$ & $181 / 18493$ & 0.005 & TGFB1 GSK3B PARP1 \\
\hline GO:0042307 & positive regulation of protein import into nucleus & $2 / 20$ & $42 / 18493$ & 0.005 & TGFB1 SMAD3 \\
\hline GO:0051147 & regulation of muscle cell differentiation & $3 / 20$ & $182 / 18493$ & 0.005 & TGFB1 BCL2 GSK3A \\
\hline GO:0061138 & morphogenesis of a branching epithelium & $3 / 20$ & $182 / 18493$ & 0.005 & PPP1CA TGFB1 BCL2 \\
\hline GO:0050864 & regulation of $\mathrm{B}$ cell activation & $3 / 20$ & $183 / 18493$ & 0.005 & TGFB1 BCL2 IL6 \\
\hline GO: 1903708 & positive regulation of hemopoiesis & $3 / 20$ & $183 / 18493$ & 0.005 & JUN STAT3 TGFB1 \\
\hline GO:0001819 & positive regulation of cytokine production & $4 / 20$ & $422 / 18493$ & 0.005 & $\begin{array}{l}\text { TGFB1 SMAD3 IRF3 } \\
\text { IL6 }\end{array}$ \\
\hline GO:0032386 & regulation of intracellular transport & $4 / 20$ & $422 / 18493$ & 0.005 & $\begin{array}{l}\text { TGFB1 SMAD3 GSK3A } \\
\text { GSK3B }\end{array}$ \\
\hline GO:0007212 & dopamine receptor signaling pathway & $2 / 20$ & $43 / 18493$ & 0.006 & GSK3A GSK3B \\
\hline GO:0032965 & regulation of collagen biosynthetic process & $2 / 20$ & $43 / 18493$ & 0.006 & TGFB1 IL6 \\
\hline GO:0050731 & $\begin{array}{l}\text { positive regulation of peptidyl-tyrosine } \\
\text { phosphorylation }\end{array}$ & $3 / 20$ & $186 / 18493$ & 0.006 & STAT3 TGFB1 IL6 \\
\hline GO:0048608 & reproductive structure development & $4 / 20$ & $428 / 18493$ & 0.006 & $\begin{array}{l}\text { TFEB PHB BCL2L1 } \\
\text { BCL2 }\end{array}$ \\
\hline GO:0002639 & positive regulation of immunoglobulin production & $2 / 20$ & $44 / 18493$ & 0.006 & TGFB1 IL6 \\
\hline GO:0070169 & positive regulation of biomineral tissue development & $2 / 20$ & $44 / 18493$ & 0.006 & TGFB1 SMAD3 \\
\hline GO:1904591 & positive regulation of protein import & $2 / 20$ & $44 / 18493$ & 0.006 & TGFB1 SMAD3 \\
\hline GO:0061458 & reproductive system development & $4 / 20$ & $431 / 18493$ & 0.006 & $\begin{array}{l}\text { TFEB PHB BCL2L1 } \\
\text { BCL2 }\end{array}$ \\
\hline GO:0001935 & endothelial cell proliferation & $3 / 20$ & $190 / 18493$ & 0.006 & STAT5A JUN STAT3 \\
\hline GO: 1901654 & response to ketone & $3 / 20$ & $190 / 18493$ & 0.006 & TGFB1 BCL2L1 PARP1 \\
\hline GO:0043401 & steroid hormone mediated signaling pathway & $3 / 20$ & $191 / 18493$ & 0.006 & PHB SKP2 PARP1 \\
\hline GO:0030857 & negative regulation of epithelial cell differentiation & $2 / 20$ & $46 / 18493$ & 0.006 & GSK3A GSK3B \\
\hline GO:0031103 & axon regeneration & $2 / 20$ & $46 / 18493$ & 0.006 & JUN BCL2 \\
\hline GO:0035196 & $\begin{array}{l}\text { production of miRNAs involved in gene silencing by } \\
\text { miRNA }\end{array}$ & $2 / 20$ & $46 / 18493$ & 0.006 & TGFB1 SMAD3 \\
\hline GO:0051168 & nuclear export & $3 / 20$ & $194 / 18493$ & 0.006 & TGFB1 GSK3B NPM1 \\
\hline GO:0002067 & glandular epithelial cell differentiation & $2 / 20$ & $47 / 18493$ & 0.006 & GSK3A GSK3B \\
\hline GO:0006953 & acute-phase response & $2 / 20$ & $47 / 18493$ & 0.006 & STAT3 IL6 \\
\hline GO:0150076 & neuroinflammatory response & $2 / 20$ & $47 / 18493$ & 0.006 & JUN IL6 \\
\hline
\end{tabular}




\begin{tabular}{|c|c|c|c|c|c|}
\hline ID & Description & GeneRatio & BgRatio & p.adjust & geneID \\
\hline GO:0001763 & morphogenesis of a branching structure & $3 / 20$ & $196 / 18493$ & 0.006 & PPP1CA TGFB1 BCL2 \\
\hline GO:0002573 & myeloid leukocyte differentiation & $3 / 20$ & $197 / 18493$ & 0.006 & JUN TGFB1 PARP1 \\
\hline GO:0003254 & regulation of membrane depolarization & $2 / 20$ & $48 / 18493$ & 0.006 & BCL2 PARP1 \\
\hline GO:0010712 & regulation of collagen metabolic process & $2 / 20$ & $48 / 18493$ & 0.006 & TGFB1 IL6 \\
\hline GO:0043525 & positive regulation of neuron apoptotic process & $2 / 20$ & $48 / 18493$ & 0.006 & JUN MCL1 \\
\hline GO:0098586 & cellular response to virus & $2 / 20$ & $48 / 18493$ & 0.006 & IRF3 BCL2L1 \\
\hline GO:2000725 & regulation of cardiac muscle cell differentiation & $2 / 20$ & $48 / 18493$ & 0.006 & TGFB1 GSK3A \\
\hline GO:0071222 & cellular response to lipopolysaccharide & $3 / 20$ & $199 / 18493$ & 0.006 & TGFB1 IRF3 IL6 \\
\hline GO:0043254 & regulation of protein complex assembly & $4 / 20$ & $451 / 18493$ & 0.007 & $\begin{array}{c}\text { JUN TGFB1 GSK3B } \\
\text { PARP1 }\end{array}$ \\
\hline GO:0010718 & $\begin{array}{l}\text { positive regulation of epithelial to mesenchymal } \\
\text { transition }\end{array}$ & $2 / 20$ & $49 / 18493$ & 0.007 & TGFB1 SMAD3 \\
\hline GO:0031050 & dsRNA processing & $2 / 20$ & $49 / 18493$ & 0.007 & TGFB1 SMAD3 \\
\hline GO:0048546 & digestive tract morphogenesis & $2 / 20$ & $49 / 18493$ & 0.007 & SMAD3 BCL2 \\
\hline GO:0070918 & $\begin{array}{c}\text { production of small RNA involved in gene silencing } \\
\text { by RNA }\end{array}$ & $2 / 20$ & $49 / 18493$ & 0.007 & TGFB1 SMAD3 \\
\hline GO:0006006 & glucose metabolic process & $3 / 20$ & $204 / 18493$ & 0.007 & $\begin{array}{l}\text { PPP1CA GSK3A } \\
\text { GSK3B }\end{array}$ \\
\hline GO:0032886 & regulation of microtubule-based process & $3 / 20$ & $204 / 18493$ & 0.007 & ATF5 GSK3B NPM1 \\
\hline GO:0001954 & positive regulation of cell-matrix adhesion & $2 / 20$ & $50 / 18493$ & 0.007 & SMAD3 GSK3B \\
\hline GO:0050670 & regulation of lymphocyte proliferation & $3 / 20$ & $205 / 18493$ & 0.007 & TGFB1 BCL2 IL6 \\
\hline GO:0032944 & regulation of mononuclear cell proliferation & $3 / 20$ & $206 / 18493$ & 0.007 & TGFB1 BCL2 IL6 \\
\hline GO:0035303 & regulation of dephosphorylation & $3 / 20$ & $206 / 18493$ & 0.007 & TGFB1 SMAD3 GSK3B \\
\hline GO:0071219 & cellular response to molecule of bacterial origin & $3 / 20$ & $206 / 18493$ & 0.007 & TGFB1 IRF3 IL6 \\
\hline GO:0016055 & Wnt signaling pathway & $4 / 20$ & $463 / 18493$ & 0.007 & $\begin{array}{l}\text { PPP1CA SMAD3 } \\
\text { GSK3A GSK3B }\end{array}$ \\
\hline GO:2000677 & $\begin{array}{c}\text { regulation of transcription regulatory region DNA } \\
\text { binding }\end{array}$ & $2 / 20$ & $51 / 18493$ & 0.007 & TGFB1 PARP1 \\
\hline GO:0198738 & cell-cell signaling by wnt & $4 / 20$ & $465 / 18493$ & 0.007 & $\begin{array}{l}\text { PPP1CA SMAD3 } \\
\text { GSK3A GSK3B }\end{array}$ \\
\hline GO:0006914 & autophagy & $4 / 20$ & $466 / 18493$ & 0.007 & $\begin{array}{l}\text { TFEB MCL1 BCL2 } \\
\text { GSK3A }\end{array}$ \\
\hline GO:0061919 & process utilizing autophagic mechanism & $4 / 20$ & $466 / 18493$ & 0.007 & $\begin{array}{l}\text { TFEB MCL1 BCL2 } \\
\text { GSK3A }\end{array}$ \\
\hline GO:0002699 & positive regulation of immune effector process & $3 / 20$ & $209 / 18493$ & 0.007 & PHB TGFB1 IL6 \\
\hline GO:0010810 & regulation of cell-substrate adhesion & $3 / 20$ & $209 / 18493$ & 0.007 & SMAD3 BCL2 GSK3B \\
\hline
\end{tabular}




\begin{tabular}{|c|c|c|c|c|c|}
\hline ID & Description & GeneRatio & BgRatio & p.adjust & geneID \\
\hline GO:0002763 & $\begin{array}{c}\text { positive regulation of myeloid leukocyte } \\
\text { differentiation }\end{array}$ & $2 / 20$ & $52 / 18493$ & 0.007 & JUN TGFB1 \\
\hline GO:0032964 & collagen biosynthetic process & $2 / 20$ & $52 / 18493$ & 0.007 & TGFB1 IL6 \\
\hline GO:0048641 & regulation of skeletal muscle tissue development & $2 / 20$ & $52 / 18493$ & 0.007 & TGFB1 BCL2 \\
\hline GO:2001169 & regulation of ATP biosynthetic process & $2 / 20$ & $52 / 18493$ & 0.007 & STAT3 PARP1 \\
\hline GO:0050920 & regulation of chemotaxis & $3 / 20$ & $210 / 18493$ & 0.007 & TGFB1 SMAD3 IL6 \\
\hline GO:0002526 & acute inflammatory response & $3 / 20$ & $211 / 18493$ & 0.007 & PHB STAT3 IL6 \\
\hline GO:0006091 & generation of precursor metabolites and energy & $4 / 20$ & $471 / 18493$ & 0.007 & $\begin{array}{c}\text { STAT3 PPP1CA GSK3A } \\
\text { GSK3B }\end{array}$ \\
\hline GO:0043331 & response to dsRNA & $2 / 20$ & $53 / 18493$ & 0.007 & IRF3 NPM1 \\
\hline GO:0001649 & osteoblast differentiation & $3 / 20$ & $214 / 18493$ & 0.007 & PHB SMAD3 IL6 \\
\hline GO:0008406 & gonad development & $3 / 20$ & $216 / 18493$ & 0.008 & PHB BCL2L1 BCL2 \\
\hline GO:0070663 & regulation of leukocyte proliferation & $3 / 20$ & $216 / 18493$ & 0.008 & TGFB1 BCL2 IL6 \\
\hline GO:0002009 & morphogenesis of an epithelium & $4 / 20$ & $479 / 18493$ & 0.008 & $\begin{array}{l}\text { PPP1CA TGFB1 } \\
\text { SMAD3 BCL2 }\end{array}$ \\
\hline GO:0043393 & regulation of protein binding & $3 / 20$ & $217 / 18493$ & 0.008 & PPP1CA BCL2 GSK3B \\
\hline GO:0044839 & cell cycle $\mathrm{G} 2 / \mathrm{M}$ phase transition & $3 / 20$ & $218 / 18493$ & 0.008 & ATF5 SKP2 NPM1 \\
\hline GO:0031102 & neuron projection regeneration & $2 / 20$ & $55 / 18493$ & 0.008 & JUN BCL2 \\
\hline GO:0048146 & positive regulation of fibroblast proliferation & $2 / 20$ & $55 / 18493$ & 0.008 & JUN TGFB1 \\
\hline GO:0051054 & positive regulation of DNA metabolic process & $3 / 20$ & $220 / 18493$ & 0.008 & JUN TGFB1 PARP1 \\
\hline GO:0031663 & lipopolysaccharide-mediated signaling pathway & $2 / 20$ & $56 / 18493$ & 0.008 & TGFB1 IRF3 \\
\hline GO:0035306 & positive regulation of dephosphorylation & $2 / 20$ & $56 / 18493$ & 0.008 & TGFB1 SMAD3 \\
\hline GO:0045843 & $\begin{array}{l}\text { negative regulation of striated muscle tissue } \\
\text { development }\end{array}$ & $2 / 20$ & $56 / 18493$ & 0.008 & TGFB1 GSK3A \\
\hline GO:0046605 & regulation of centrosome cycle & $2 / 20$ & $56 / 18493$ & 0.008 & ATF5 NPM1 \\
\hline GO: 1903749 & $\begin{array}{l}\text { positive regulation of establishment of protein } \\
\text { localization to mitochondrion }\end{array}$ & $2 / 20$ & $56 / 18493$ & 0.008 & BCL2 GSK3A \\
\hline GO:0007160 & cell-matrix adhesion & $3 / 20$ & $222 / 18493$ & 0.008 & SMAD3 BCL2 GSK3B \\
\hline GO:0045137 & development of primary sexual characteristics & $3 / 20$ & $222 / 18493$ & 0.008 & PHB BCL2L1 BCL2 \\
\hline GO:2001234 & negative regulation of apoptotic signaling pathway & $3 / 20$ & $222 / 18493$ & 0.008 & BCL2L1 MCL1 BCL2 \\
\hline GO:0002066 & columnar/cuboidal epithelial cell development & $2 / 20$ & $57 / 18493$ & 0.008 & GSK3A GSK3B \\
\hline GO:0042093 & T-helper cell differentiation & $2 / 20$ & $57 / 18493$ & 0.008 & STAT3 IL6 \\
\hline GO:0048635 & negative regulation of muscle organ development & $2 / 20$ & $57 / 18493$ & 0.008 & TGFB1 GSK3A \\
\hline
\end{tabular}




\begin{tabular}{|c|c|c|c|c|c|}
\hline ID & Description & GeneRatio & BgRatio & p.adjust & geneID \\
\hline GO:0000302 & response to reactive oxygen species & $3 / 20$ & $224 / 18493$ & 0.008 & JUN BCL2 IL6 \\
\hline GO:0030520 & intracellular estrogen receptor signaling pathway & $2 / 20$ & $58 / 18493$ & 0.008 & SKP2 PARP1 \\
\hline GO:0043388 & positive regulation of DNA binding & $2 / 20$ & $58 / 18493$ & 0.008 & TGFB1 PARP1 \\
\hline GO: 1901862 & negative regulation of muscle tissue development & $2 / 20$ & $58 / 18493$ & 0.008 & TGFB1 GSK3A \\
\hline GO:2000378 & $\begin{array}{l}\text { negative regulation of reactive oxygen species } \\
\text { metabolic process }\end{array}$ & $2 / 20$ & $58 / 18493$ & 0.008 & STAT3 BCL2 \\
\hline GO:0002294 & $\begin{array}{l}\text { CD4-positive, alpha-beta } \mathrm{T} \text { cell differentiation } \\
\text { involved in immune response }\end{array}$ & $2 / 20$ & $59 / 18493$ & 0.009 & STAT3 IL6 \\
\hline GO:0001658 & branching involved in ureteric bud morphogenesis & $2 / 20$ & $60 / 18493$ & 0.009 & TGFB1 BCL2 \\
\hline GO:0002287 & $\begin{array}{c}\text { alpha-beta } \mathrm{T} \text { cell activation involved in immune } \\
\text { response }\end{array}$ & $2 / 20$ & $60 / 18493$ & 0.009 & STAT3 IL6 \\
\hline GO:0002293 & $\begin{array}{c}\text { alpha-beta } \mathrm{T} \text { cell differentiation involved in immune } \\
\text { response }\end{array}$ & $2 / 20$ & $60 / 18493$ & 0.009 & STAT3 IL6 \\
\hline GO:0090092 & $\begin{array}{l}\text { regulation of transmembrane receptor protein } \\
\text { serine/threonine kinase signaling pathway }\end{array}$ & $3 / 20$ & $235 / 18493$ & 0.009 & TGFB1 SMAD3 PARP1 \\
\hline GO:0009152 & purine ribonucleotide biosynthetic process & $3 / 20$ & $239 / 18493$ & 0.01 & STAT3 TGFB1 PARP1 \\
\hline GO:0002637 & regulation of immunoglobulin production & $2 / 20$ & $63 / 18493$ & 0.01 & TGFB1 IL6 \\
\hline GO:0009755 & hormone-mediated signaling pathway & $3 / 20$ & $240 / 18493$ & 0.01 & PHB SKP2 PARP1 \\
\hline GO:0002260 & lymphocyte homeostasis & $2 / 20$ & $64 / 18493$ & 0.01 & TGFB1 BCL2 \\
\hline GO:0042306 & regulation of protein import into nucleus & $2 / 20$ & $64 / 18493$ & 0.01 & TGFB1 SMAD3 \\
\hline GO:1905207 & regulation of cardiocyte differentiation & $2 / 20$ & $64 / 18493$ & 0.01 & TGFB1 GSK3A \\
\hline GO:0019318 & hexose metabolic process & $3 / 20$ & $244 / 18493$ & 0.01 & $\begin{array}{l}\text { PPP1CA GSK3A } \\
\text { GSK3B }\end{array}$ \\
\hline GO:0050730 & regulation of peptidyl-tyrosine phosphorylation & $3 / 20$ & $245 / 18493$ & 0.01 & STAT3 TGFB1 IL6 \\
\hline GO:0070374 & positive regulation of ERK1 and ERK2 cascade & $3 / 20$ & $245 / 18493$ & 0.01 & PHB JUN TGFB1 \\
\hline GO:0051926 & negative regulation of calcium ion transport & $2 / 20$ & $65 / 18493$ & 0.01 & TGFB1 BCL2 \\
\hline GO:0060675 & ureteric bud morphogenesis & $2 / 20$ & $65 / 18493$ & 0.01 & TGFB1 BCL2 \\
\hline GO: 1904888 & cranial skeletal system development & $2 / 20$ & $65 / 18493$ & 0.01 & TGFB1 SMAD3 \\
\hline
\end{tabular}




\section{PATHWAY ENRICHMENT:}

\section{HCOV-HOST_SET}




\subsection{KEGG PATHWAY ENRICHMENT}

\section{BARPLOT}

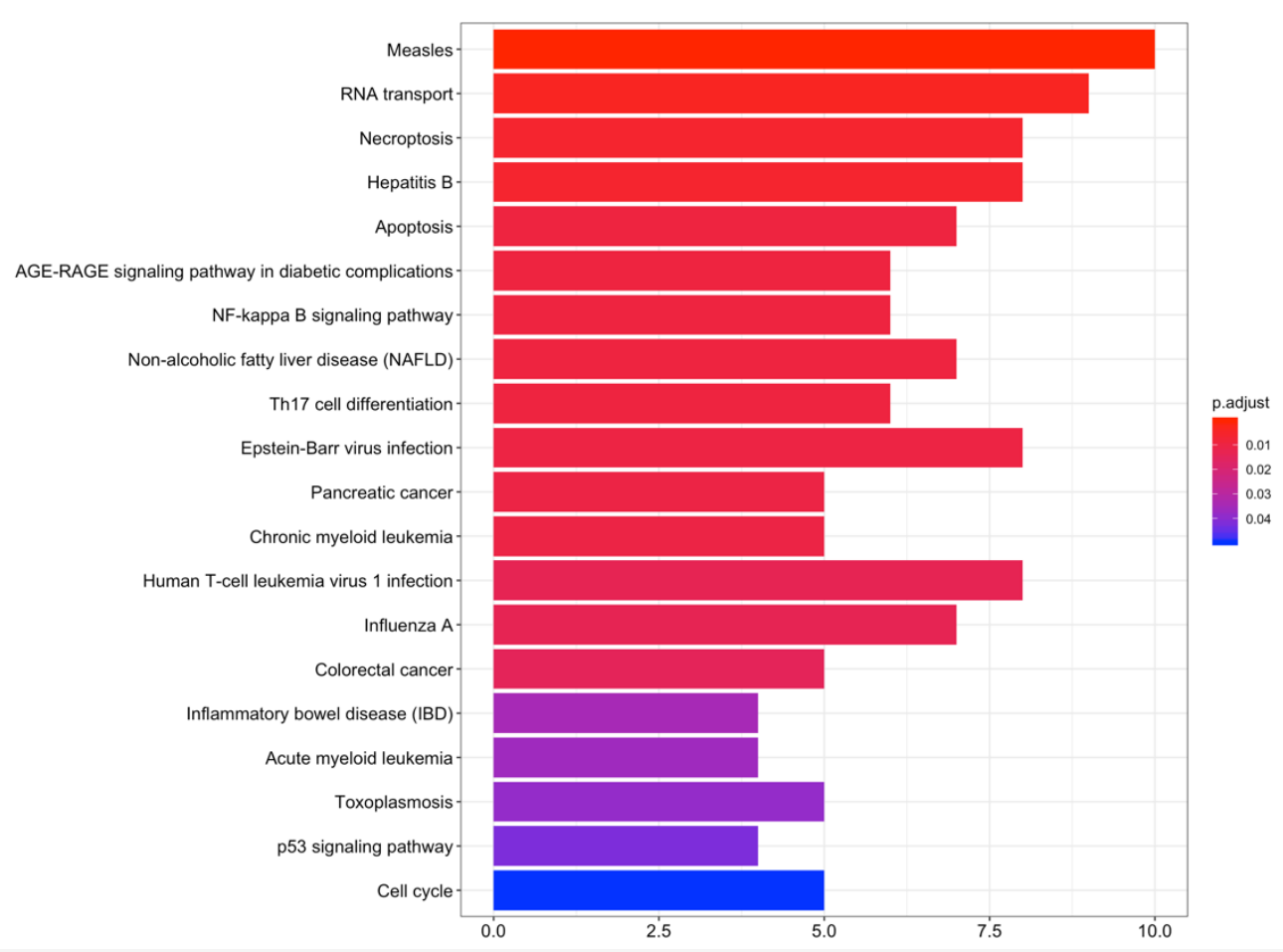

NETPLOT
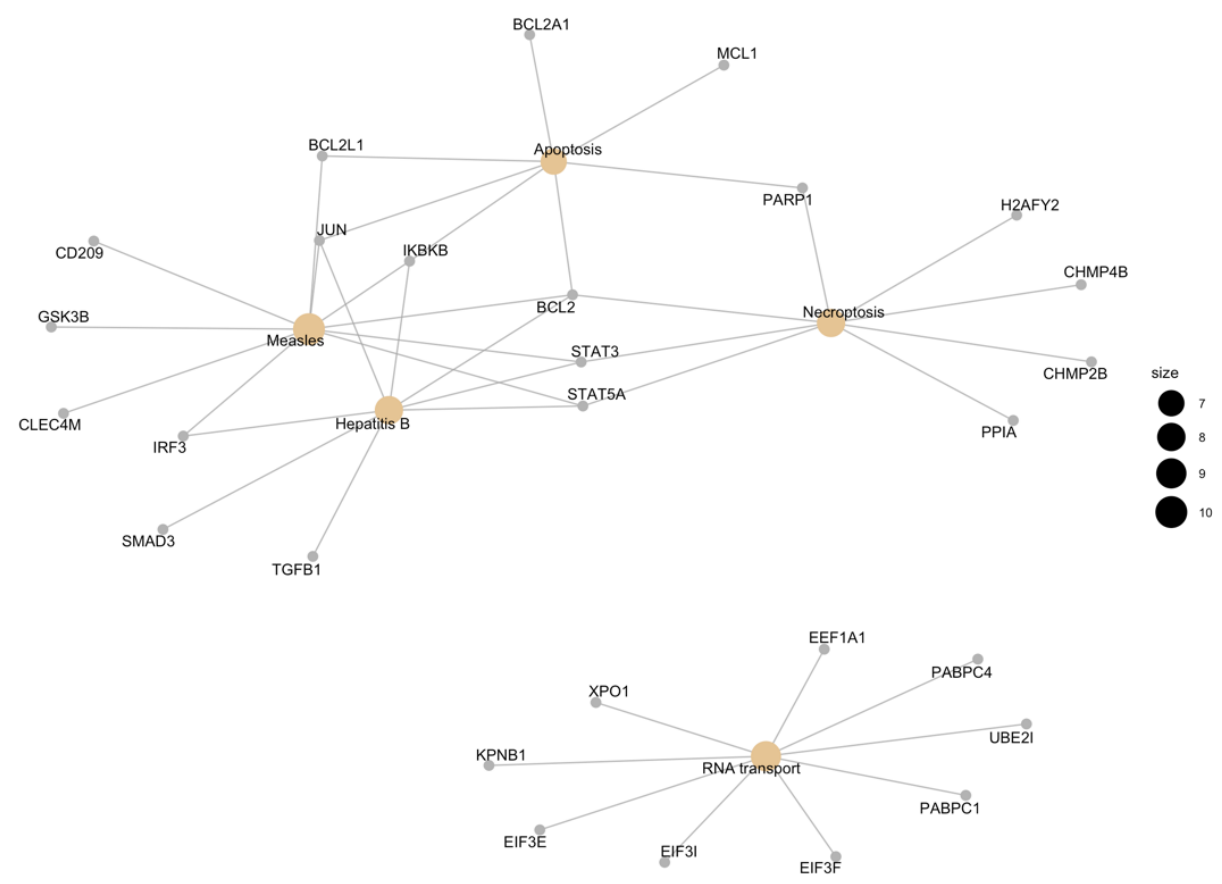


\begin{tabular}{|c|c|c|c|c|c|}
\hline ID & Description & GeneRatio & BgRatio & p.adjust & geneID \\
\hline hsa05162 & Measles & $10 / 76$ & $138 / 8018$ & 0 & $\begin{array}{l}\text { STAT5A JUN STAT3 CD209 CLEC4M } \\
\text { IRF3 BCL2L1 BCL2 IKBKB GSK3B }\end{array}$ \\
\hline hsa03013 & RNA transport & $9 / 76$ & $179 / 8018$ & 0.004 & $\begin{array}{l}\text { KPNB1 XPO1 EIF3E EIF3I EIF3F } \\
\text { PABPC1 PABPC4 EEF1A1 UBE2I }\end{array}$ \\
\hline hsa04217 & Necroptosis & $8 / 76$ & $159 / 8018$ & 0.006 & $\begin{array}{c}\text { STAT5A STAT3 PPIA BCL2 CHMP4B } \\
\text { PARP1 CHMP2B H2AFY2 }\end{array}$ \\
\hline hsa05161 & Hepatitis B & $8 / 76$ & $162 / 8018$ & 0.006 & $\begin{array}{c}\text { STAT5A JUN STAT3 TGFB1 SMAD3 } \\
\text { IRF3 BCL2 IKBKB }\end{array}$ \\
\hline hsa04210 & Apoptosis & $7 / 76$ & $136 / 8018$ & 0.01 & $\begin{array}{l}\text { JUN BCL2L1 MCL1 BCL2A1 BCL2 } \\
\text { IKBKB PARP1 }\end{array}$ \\
\hline hsa04933 & $\begin{array}{l}\text { AGE-RAGE signaling pathway in } \\
\text { diabetic complications }\end{array}$ & $6 / 76$ & $100 / 8018$ & 0.01 & $\begin{array}{l}\text { STAT5A JUN STAT3 TGFB1 SMAD3 } \\
\text { BCL2 }\end{array}$ \\
\hline hsa04064 & NF-kappa B signaling pathway & $6 / 76$ & $104 / 8018$ & 0.01 & $\begin{array}{l}\text { BCL2L1 BCL2A1 BCL2 IKBKB PARP1 } \\
\text { UBE2I }\end{array}$ \\
\hline hsa04932 & $\begin{array}{l}\text { Non-alcoholic fatty liver disease } \\
\text { (NAFLD) }\end{array}$ & $7 / 76$ & $149 / 8018$ & 0.01 & $\begin{array}{l}\text { JUN TGFB1 COX2 IKBKB GSK3A } \\
\text { GSK3B NDUFA10 }\end{array}$ \\
\hline hsa04659 & Th17 cell differentiation & $6 / 76$ & $107 / 8018$ & 0.01 & $\begin{array}{l}\text { STAT5A JUN STAT3 TGFB1 SMAD3 } \\
\text { IKBKB }\end{array}$ \\
\hline hsa05169 & Epstein-Barr virus infection & $8 / 76$ & $201 / 8018$ & 0.01 & $\begin{array}{l}\text { PSMC2 JUN STAT3 IRF3 BCL2 SKP2 } \\
\text { IKBKB PSMD1 }\end{array}$ \\
\hline hsa05212 & Pancreatic cancer & $5 / 76$ & $76 / 8018$ & 0.01 & STAT3 TGFB1 SMAD3 BCL2L1 IKBKB \\
\hline hsa05220 & Chronic myeloid leukemia & $5 / 76$ & $76 / 8018$ & 0.01 & $\begin{array}{l}\text { STAT5A TGFB1 SMAD3 BCL2L1 } \\
\text { IKBKB }\end{array}$ \\
\hline hsa05166 & Human T-cell leukemia virus 1 infection & $8 / 76$ & $219 / 8018$ & 0.013 & $\begin{array}{l}\text { STAT5A JUN XPO1 TGFB1 SMAD3 } \\
\text { BCL2L1 IKBKB CHEK2 }\end{array}$ \\
\hline hsa05164 & Influenza A & $7 / 76$ & $170 / 8018$ & 0.013 & $\begin{array}{c}\text { XPO1 IRF3 KPNA2 TMPRSS2 IKBKB } \\
\text { DNAJB1 TPSAB1 }\end{array}$ \\
\hline hsa05210 & Colorectal cancer & $5 / 76$ & $86 / 8018$ & 0.015 & JUN TGFB1 SMAD3 BCL2 GSK3B \\
\hline hsa05321 & Inflammatory bowel disease (IBD) & $4 / 76$ & $65 / 8018$ & 0.035 & JUN STAT3 TGFB1 SMAD3 \\
\hline hsa05221 & Acute myeloid leukemia & $4 / 76$ & $67 / 8018$ & 0.036 & STAT5A STAT3 BCL2A1 IKBKB \\
\hline hsa05145 & Toxoplasmosis & $5 / 76$ & $112 / 8018$ & 0.039 & STAT3 TGFB1 BCL2L1 BCL2 IKBKB \\
\hline hsa04115 & p53 signaling pathway & $4 / 76$ & $72 / 8018$ & 0.042 & BCL2L1 BCL2 RRM2 CHEK2 \\
\hline hsa04110 & Cell cycle & $5 / 76$ & $124 / 8018$ & 0.05 & TGFB1 SMAD3 SKP2 GSK3B CHEK2 \\
\hline hsa01521 & $\begin{array}{l}\text { EGFR tyrosine kinase inhibitor } \\
\text { resistance }\end{array}$ & $4 / 76$ & $79 / 8018$ & 0.05 & STAT3 BCL2L1 BCL2 GSK3B \\
\hline hsa03018 & RNA degradation & $4 / 76$ & $79 / 8018$ & 0.05 & HSPD1 HSPA9 PABPC1 PABPC4 \\
\hline hsa05131 & Shigellosis & $7 / 76$ & $236 / 8018$ & 0.05 & $\begin{array}{l}\text { JUN IRF3 BCL2L1 BCL2 IKBKB } \\
\text { GSK3A GSK3B }\end{array}$ \\
\hline hsa05152 & Tuberculosis & $6 / 76$ & $180 / 8018$ & 0.05 & $\begin{array}{c}\text { TGFB1 CD209 CLEC4M BCL2 HSPD1 } \\
\text { HSPA9 }\end{array}$ \\
\hline
\end{tabular}




\subsection{WIKI PATHWAY}

\section{BARPLOT}

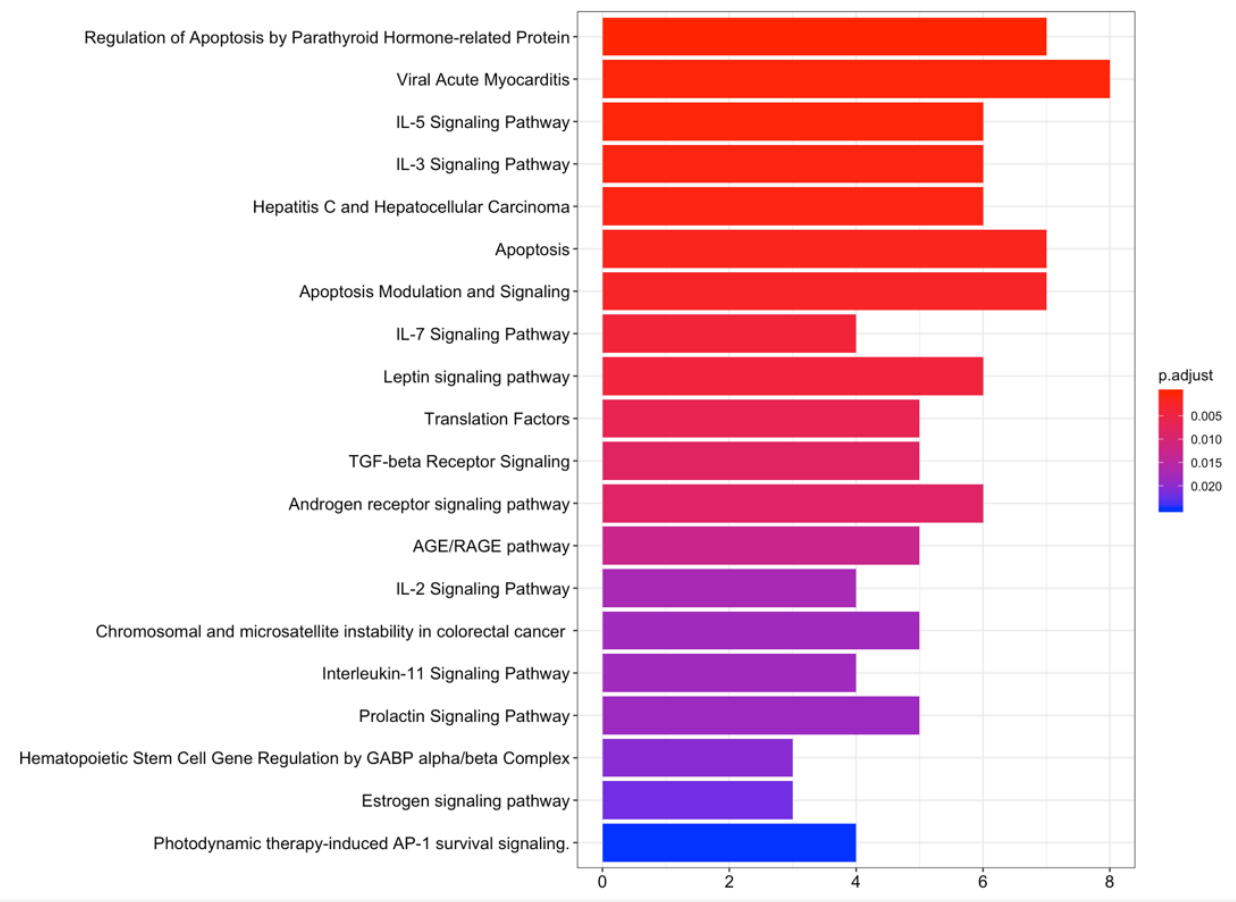

NETPLOT

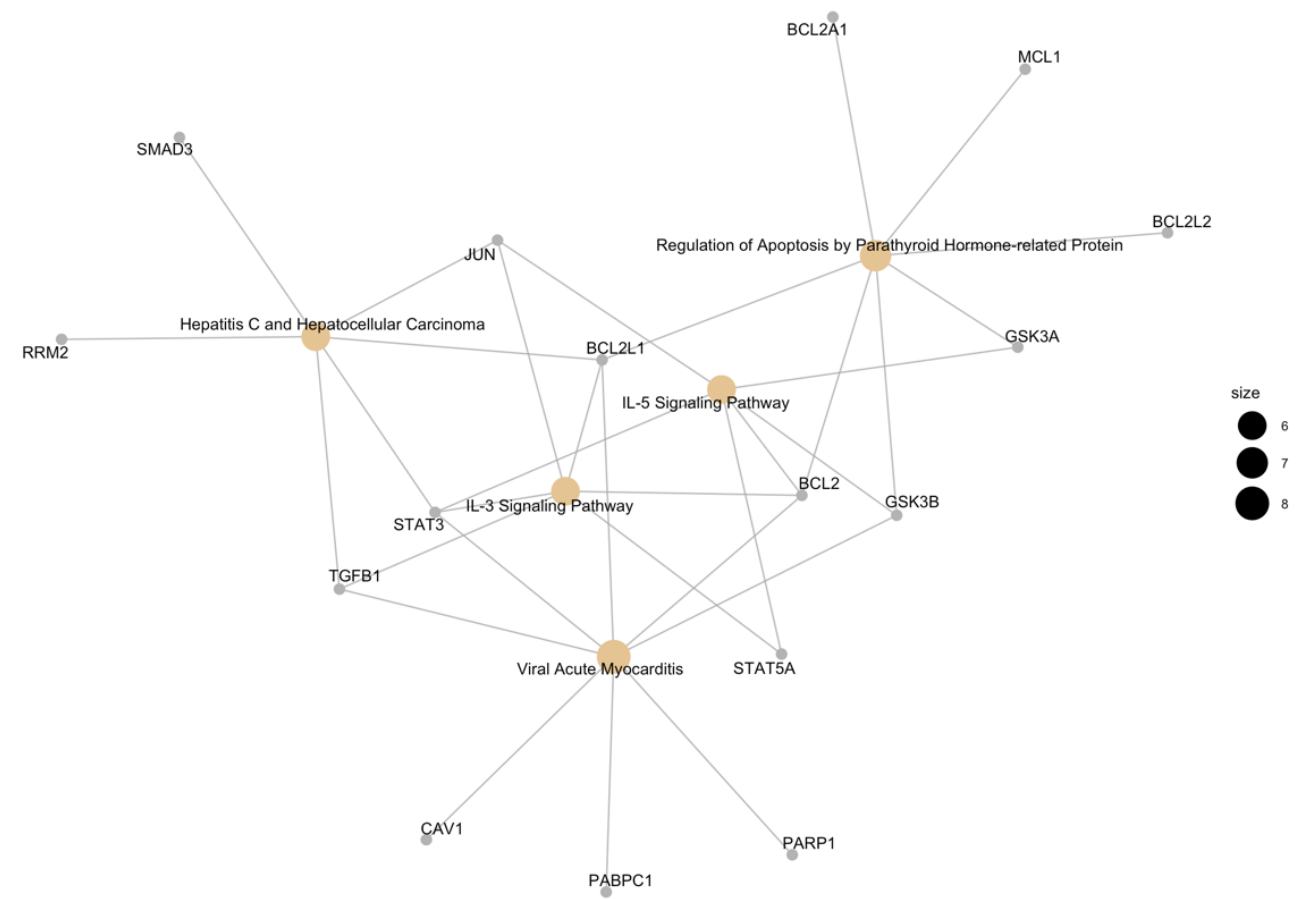




\begin{tabular}{|c|c|c|c|c|c|}
\hline ID & Description & GeneRatio & BgRatio & p.adjust & geneID \\
\hline WP3872 & $\begin{array}{c}\text { Regulation of Apoptosis by Parathyroid Hormone- } \\
\text { related Protein }\end{array}$ & $7 / 70$ & $22 / 6249$ & 0 & $\begin{array}{c}\text { BCL2L1 BCL2L2 MCL1 } \\
\text { BCL2A1 BCL2 GSK3A GSK3B }\end{array}$ \\
\hline WP4298 & Viral Acute Myocarditis & $8 / 70$ & $85 / 6249$ & 0 & $\begin{array}{l}\text { STAT3 TGFB1 BCL2L1 BCL2 } \\
\text { GSK3B PABPC1 PARP1 CAV1 }\end{array}$ \\
\hline WP127 & IL-5 Signaling Pathway & $6 / 70$ & $40 / 6249$ & 0 & $\begin{array}{c}\text { STAT5A JUN STAT3 BCL2 } \\
\text { GSK3A GSK3B }\end{array}$ \\
\hline WP286 & IL-3 Signaling Pathway & $6 / 70$ & $49 / 6249$ & 0.001 & $\begin{array}{l}\text { STAT5A JUN STAT3 TGFB1 } \\
\text { BCL2L1 BCL2 }\end{array}$ \\
\hline WP3646 & Hepatitis C and Hepatocellular Carcinoma & $6 / 70$ & $51 / 6249$ & 0.001 & $\begin{array}{l}\text { JUN STAT3 TGFB1 SMAD3 } \\
\text { BCL2L1 RRM2 }\end{array}$ \\
\hline WP254 & Apoptosis & $7 / 70$ & $86 / 6249$ & 0.001 & $\begin{array}{l}\text { JUN IRF3 BCL2L1 BCL2L2 } \\
\text { MCL1 BCL2 IKBKB }\end{array}$ \\
\hline WP1772 & Apoptosis Modulation and Signaling & $7 / 70$ & $94 / 6249$ & 0.002 & $\begin{array}{l}\text { JUN BCL2L1 BCL2L2 MCL1 } \\
\text { BCL2A1 BCL2 IKBKB }\end{array}$ \\
\hline WP205 & IL-7 Signaling Pathway & $4 / 70$ & $25 / 6249$ & 0.004 & $\begin{array}{l}\text { STAT5A STAT3 BCL2L1 } \\
\text { GSK3B }\end{array}$ \\
\hline WP2034 & Leptin signaling pathway & $6 / 70$ & $76 / 6249$ & 0.004 & $\begin{array}{c}\text { STAT3 BCL2L1 KPNA4 IKBKB } \\
\text { GSK3A GSK3B }\end{array}$ \\
\hline WP107 & Translation Factors & $5 / 70$ & $54 / 6249$ & 0.006 & $\begin{array}{l}\text { EIF3E EIF3I EIF3F PABPC1 } \\
\text { EEF1A1 }\end{array}$ \\
\hline WP560 & TGF-beta Receptor Signaling & $5 / 70$ & $58 / 6249$ & 0.008 & $\begin{array}{l}\text { JUN STAT3 TGFB1 SMAD3 } \\
\text { FKBP1A }\end{array}$ \\
\hline WP138 & Androgen receptor signaling pathway & $6 / 70$ & $91 / 6249$ & 0.009 & $\begin{array}{l}\text { JUN STAT3 SMAD3 GSK3B } \\
\text { CAV1 UBE2I }\end{array}$ \\
\hline WP2324 & AGE/RAGE pathway & $5 / 70$ & $66 / 6249$ & 0.013 & $\begin{array}{l}\text { STAT5A JUN STAT3 SMAD3 } \\
\text { IKBKB }\end{array}$ \\
\hline WP49 & IL-2 Signaling Pathway & $4 / 70$ & $42 / 6249$ & 0.017 & STAT5A JUN STAT3 BCL2 \\
\hline WP4216 & $\begin{array}{l}\text { Chromosomal and microsatellite instability in } \\
\text { colorectal cancer }\end{array}$ & $5 / 70$ & $74 / 6249$ & 0.018 & $\begin{array}{l}\text { JUN TGFB1 SMAD3 BCL2 } \\
\text { GSK3B }\end{array}$ \\
\hline WP2332 & Interleukin-11 Signaling Pathway & $4 / 70$ & $44 / 6249$ & 0.018 & STAT3 TGFB1 BCL2 IKBKB \\
\hline WP2037 & Prolactin Signaling Pathway & $5 / 70$ & $76 / 6249$ & 0.018 & $\begin{array}{l}\text { STAT5A JUN STAT3 PPIA } \\
\text { GSK3B }\end{array}$ \\
\hline WP3657 & $\begin{array}{l}\text { Hematopoietic Stem Cell Gene Regulation by } \\
\text { GABP alpha/beta Complex }\end{array}$ & $3 / 70$ & $22 / 6249$ & 0.02 & BCL2L1 MCL1 BCL2 \\
\hline WP712 & Estrogen signaling pathway & $3 / 70$ & $23 / 6249$ & 0.022 & JUN BCL2 IKBKB \\
\hline WP3611 & $\begin{array}{l}\text { Photodynamic therapy-induced AP-1 survival } \\
\text { signaling. }\end{array}$ & $4 / 70$ & $51 / 6249$ & 0.025 & JUN BCL2L1 MCL1 BCL2 \\
\hline WP4263 & Pancreatic adenocarcinoma pathway & $5 / 70$ & $89 / 6249$ & 0.029 & $\begin{array}{l}\text { STAT3 TGFB1 SMAD3 BCL2L1 } \\
\text { IKBKB }\end{array}$ \\
\hline WP4217 & Ebola Virus Pathway on Host & $6 / 70$ & $129 / 6249$ & 0.029 & $\begin{array}{c}\text { CLEC4G CD209 CLEC4M IRF3 } \\
\text { PRKRA CAV1 }\end{array}$ \\
\hline WP366 & TGF-beta Signaling Pathway & $6 / 70$ & $133 / 6249$ & 0.031 & $\begin{array}{l}\text { JUN TGFB1 SMAD3 HGS } \\
\text { CAV1 UBE2I }\end{array}$ \\
\hline
\end{tabular}




\begin{tabular}{|c|c|c|c|c|c|}
\hline ID & Description & GeneRatio & BgRatio & p.adjust & geneID \\
\hline WP411 & mRNA Processing & $6 / 70$ & $133 / 6249$ & 0.031 & $\begin{array}{l}\text { HNRNPA1 PTBP1 HNRNPA2B1 } \\
\text { SRP54 NONO DDX1 }\end{array}$ \\
\hline WP3972 & PDGFR-beta pathway & $3 / 70$ & $29 / 6249$ & 0.033 & STAT5A JUN STAT3 \\
\hline WP3888 & VEGFA-VEGFR2 Signaling Pathway & $8 / 70$ & $238 / 6249$ & 0.037 & $\begin{array}{l}\text { JUN STAT3 BCL2L1 BCL2 } \\
\text { GSK3B NCL HGS CAV1 }\end{array}$ \\
\hline WP2112 & IL17 signaling pathway & $3 / 70$ & $31 / 6249$ & 0.037 & STAT3 IKBKB GSK3B \\
\hline WP615 & Senescence and Autophagy in Cancer & $5 / 70$ & $106 / 6249$ & 0.047 & $\begin{array}{l}\text { JUN TGFB1 SMAD3 BCL2 } \\
\text { GSK3B }\end{array}$ \\
\hline WP3617 & $\begin{array}{l}\text { Photodynamic therapy-induced NF-kB survival } \\
\text { signaling }\end{array}$ & $3 / 70$ & $35 / 6249$ & 0.048 & BCL2L2 BCL2A1 IKBKB \\
\hline
\end{tabular}




\section{HCOV-CLUSTER_SET}

\subsection{KEGG PATHWAY ENRICHMENT}

\section{BARPLOT}

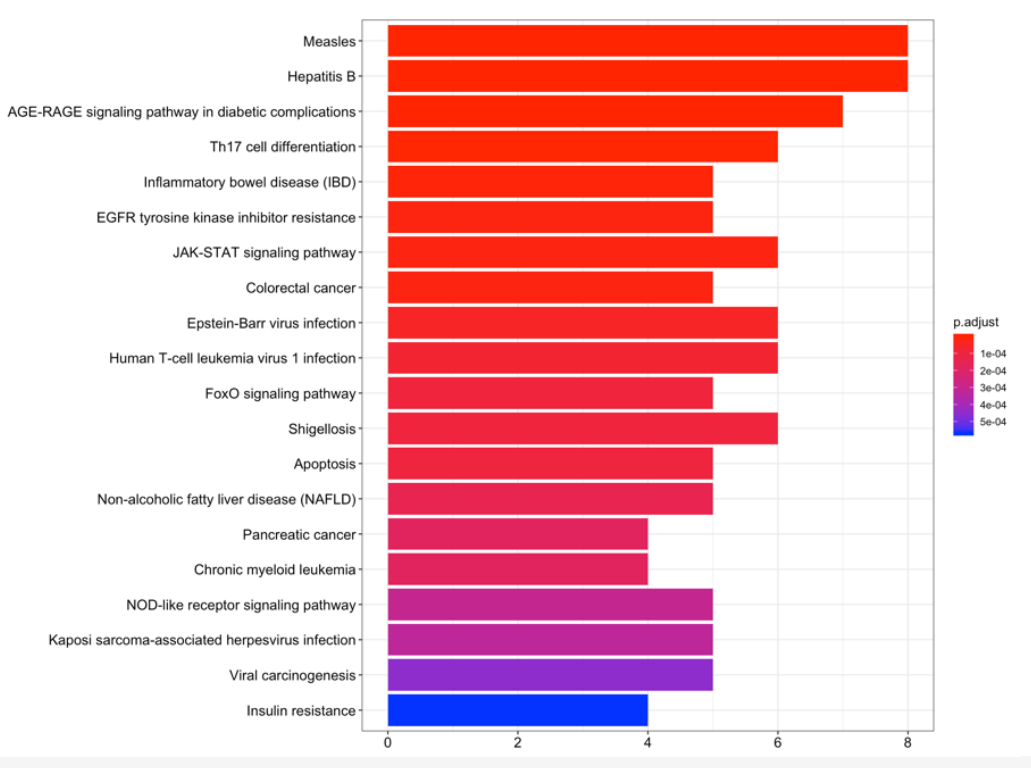

\section{NETPLOT}

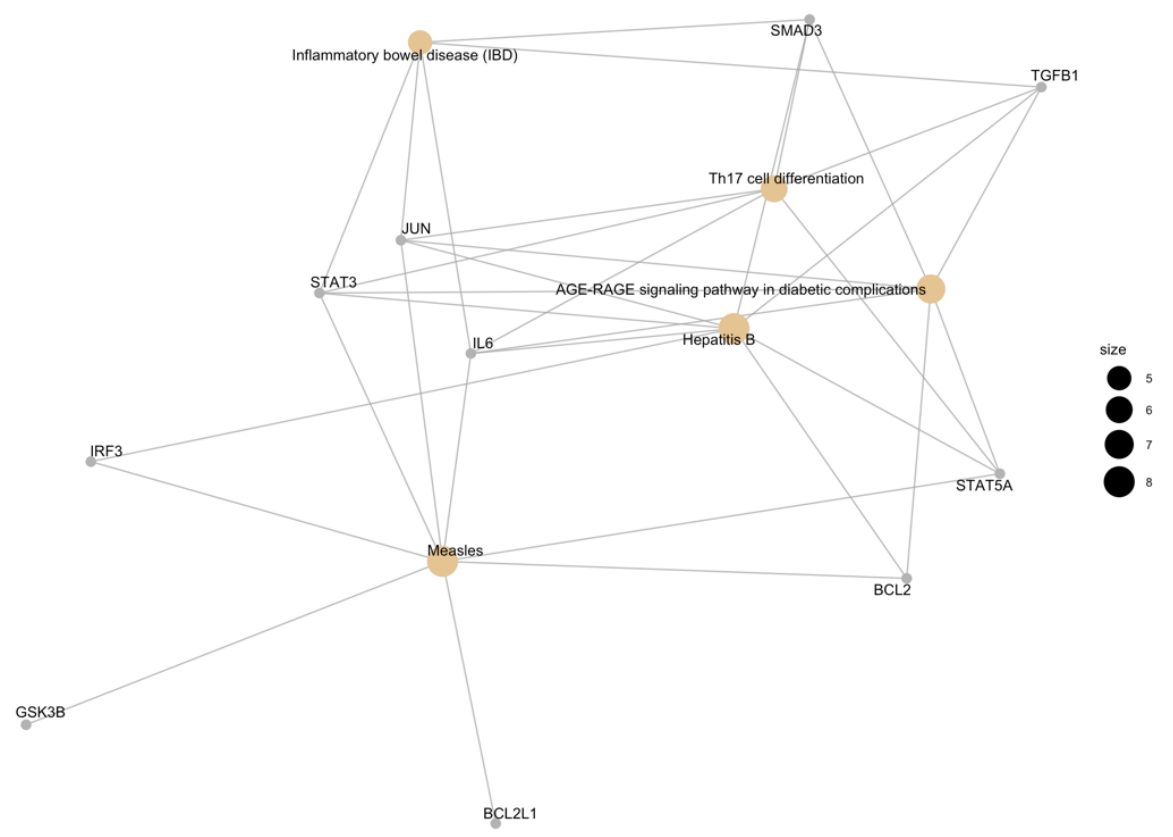




\begin{tabular}{|c|c|c|c|c|c|}
\hline ID & Description & GeneRatio & BgRatio & p.adjust & geneID \\
\hline hsa05162 & Measles & $8 / 18$ & $138 / 8018$ & 0 & $\begin{array}{l}\text { STAT5A JUN STAT3 IRF3 } \\
\text { BCL2L1 BCL2 GSK3B IL6 }\end{array}$ \\
\hline hsa05161 & Hepatitis B & $8 / 18$ & $162 / 8018$ & 0 & $\begin{array}{l}\text { STAT5A JUN STAT3 TGFB1 } \\
\text { SMAD3 IRF3 BCL2 IL6 }\end{array}$ \\
\hline hsa04933 & $\begin{array}{l}\text { AGE-RAGE signaling pathway in diabetic } \\
\text { complications }\end{array}$ & $7 / 18$ & $100 / 8018$ & 0 & $\begin{array}{l}\text { STAT5A JUN STAT3 TGFB1 } \\
\text { SMAD3 BCL2 IL6 }\end{array}$ \\
\hline hsa04659 & Th17 cell differentiation & $6 / 18$ & $107 / 8018$ & 0 & $\begin{array}{l}\text { STAT5A JUN STAT3 TGFB1 } \\
\text { SMAD3 IL6 }\end{array}$ \\
\hline hsa05321 & Inflammatory bowel disease (IBD) & $5 / 18$ & $65 / 8018$ & 0 & JUN STAT3 TGFB1 SMAD3 IL6 \\
\hline hsa01521 & EGFR tyrosine kinase inhibitor resistance & $5 / 18$ & $79 / 8018$ & 0 & STAT3 BCL2L1 BCL2 GSK3B IL6 \\
\hline hsa04630 & JAK-STAT signaling pathway & $6 / 18$ & $162 / 8018$ & 0 & $\begin{array}{c}\text { STAT5A STAT3 BCL2L1 MCL1 } \\
\text { BCL2 IL6 }\end{array}$ \\
\hline hsa05210 & Colorectal cancer & $5 / 18$ & $86 / 8018$ & 0 & $\begin{array}{c}\text { JUN TGFB1 SMAD3 BCL2 } \\
\text { GSK3B }\end{array}$ \\
\hline hsa05169 & Epstein-Barr virus infection & $6 / 18$ & $201 / 8018$ & 0 & JUN STAT3 IRF3 BCL2 SKP2 IL6 \\
\hline hsa05166 & Human T-cell leukemia virus 1 infection & $6 / 18$ & $219 / 8018$ & 0 & $\begin{array}{l}\text { STAT5A JUN TGFB1 SMAD3 } \\
\text { BCL2L1 IL6 }\end{array}$ \\
\hline hsa04068 & FoxO signaling pathway & $5 / 18$ & $131 / 8018$ & 0 & STAT3 TGFB1 SMAD3 SKP2 IL6 \\
\hline hsa05131 & Shigellosis & $6 / 18$ & $236 / 8018$ & 0 & $\begin{array}{c}\text { JUN IRF3 BCL2L1 BCL2 GSK3A } \\
\text { GSK3B }\end{array}$ \\
\hline hsa04210 & Apoptosis & $5 / 18$ & $136 / 8018$ & 0 & JUN BCL2L1 MCL1 BCL2 PARP1 \\
\hline hsa04932 & Non-alcoholic fatty liver disease (NAFLD) & $5 / 18$ & $149 / 8018$ & 0 & JUN TGFB1 GSK3A GSK3B IL6 \\
\hline hsa05212 & Pancreatic cancer & $4 / 18$ & $76 / 8018$ & 0 & STAT3 TGFB1 SMAD3 BCL2L1 \\
\hline hsa05220 & Chronic myeloid leukemia & $4 / 18$ & $76 / 8018$ & 0 & STAT5A TGFB1 SMAD3 BCL2L1 \\
\hline hsa04621 & NOD-like receptor signaling pathway & $5 / 18$ & $181 / 8018$ & 0 & JUN IRF3 BCL2L1 BCL2 IL6 \\
\hline hsa05167 & $\begin{array}{l}\text { Kaposi sarcoma-associated herpesvirus } \\
\text { infection }\end{array}$ & $5 / 18$ & $186 / 8018$ & 0 & JUN STAT3 IRF3 GSK3B IL6 \\
\hline hsa05203 & Viral carcinogenesis & $5 / 18$ & $201 / 8018$ & 0 & STAT5A JUN STAT3 IRF3 SKP2 \\
\hline hsa04931 & Insulin resistance & $4 / 18$ & $108 / 8018$ & 0.001 & STAT3 PPP1CA GSK3B IL6 \\
\hline hsa05145 & Toxoplasmosis & $4 / 18$ & $112 / 8018$ & 0.001 & STAT3 TGFB1 BCL2L1 BCL2 \\
\hline hsa05135 & Yersinia infection & $4 / 18$ & $120 / 8018$ & 0.001 & JUN IRF3 GSK3B IL6 \\
\hline hsa04110 & Cell cycle & $4 / 18$ & $124 / 8018$ & 0.001 & TGFB1 SMAD3 SKP2 GSK3B \\
\hline hsa05226 & Gastric cancer & $4 / 18$ & $149 / 8018$ & 0.002 & TGFB1 SMAD3 BCL2 GSK3B \\
\hline hsa04390 & Hippo signaling pathway & $4 / 18$ & $157 / 8018$ & 0.002 & PPP1CA TGFB1 SMAD3 GSK3B \\
\hline hsa04217 & Necroptosis & $4 / 18$ & $159 / 8018$ & 0.002 & STAT5A STAT3 BCL2 PARP1 \\
\hline hsa04218 & Cellular senescence & $4 / 18$ & $160 / 8018$ & 0.002 & PPP1CA TGFB1 SMAD3 IL6 \\
\hline
\end{tabular}




\begin{tabular}{|c|c|c|c|c|c|}
\hline ID & Description & GeneRatio & BgRatio & p.adjust & geneID \\
\hline hsa04137 & Mitophagy - animal & $3 / 18$ & $65 / 8018$ & 0.002 & TFEB JUN BCL2L1 \\
\hline hsa05225 & Hepatocellular carcinoma & $4 / 18$ & $168 / 8018$ & 0.002 & TGFB1 SMAD3 BCL2L1 GSK3B \\
\hline hsa04917 & Prolactin signaling pathway & $3 / 18$ & $70 / 8018$ & 0.002 & STAT5A STAT3 GSK3B \\
\hline hsa05133 & Pertussis & $3 / 18$ & $76 / 8018$ & 0.003 & JUN IRF3 IL6 \\
\hline hsa04012 & ErbB signaling pathway & $3 / 18$ & $85 / 8018$ & 0.004 & STAT5A JUN GSK3B \\
\hline hsa04510 & Focal adhesion & $4 / 18$ & $199 / 8018$ & 0.004 & JUN PPP1CA BCL2 GSK3B \\
\hline hsa04151 & PI3K-Akt signaling pathway & $5 / 18$ & $354 / 8018$ & 0.004 & BCL2L1 MCL1 BCL2 GSK3B IL6 \\
\hline hsa05222 & Small cell lung cancer & $3 / 18$ & $92 / 8018$ & 0.004 & BCL2L1 BCL2 SKP2 \\
\hline hsa05170 & Human immunodeficiency virus 1 infection & $4 / 18$ & $212 / 8018$ & 0.004 & JUN IRF3 BCL2L1 BCL2 \\
\hline hsa05323 & Rheumatoid arthritis & $3 / 18$ & $93 / 8018$ & 0.004 & JUN TGFB1 IL6 \\
\hline hsa04657 & IL-17 signaling pathway & $3 / 18$ & $94 / 8018$ & 0.004 & JUN GSK3B IL6 \\
\hline hsa05163 & Human cytomegalovirus infection & $4 / 18$ & $225 / 8018$ & 0.005 & STAT3 IRF3 GSK3B IL6 \\
\hline hsa05142 & Chagas disease (American trypanosomiasis) & $3 / 18$ & $102 / 8018$ & 0.005 & JUN TGFB1 IL6 \\
\hline hsa04064 & NF-kappa B signaling pathway & $3 / 18$ & $104 / 8018$ & 0.005 & BCL2L1 BCL2 PARP1 \\
\hline hsa04620 & Toll-like receptor signaling pathway & $3 / 18$ & $104 / 8018$ & 0.005 & JUN IRF3 IL6 \\
\hline hsa04066 & HIF-1 signaling pathway & $3 / 18$ & $109 / 8018$ & 0.006 & STAT3 BCL2 IL6 \\
\hline hsa04722 & Neurotrophin signaling pathway & $3 / 18$ & $119 / 8018$ & 0.007 & JUN BCL2 GSK3B \\
\hline hsa04935 & $\begin{array}{l}\text { Growth hormone synthesis, secretion and } \\
\text { action }\end{array}$ & $3 / 18$ & $119 / 8018$ & 0.007 & STAT5A STAT3 GSK3B \\
\hline hsa04215 & Apoptosis - multiple species & $2 / 18$ & $32 / 8018$ & 0.007 & BCL2L1 BCL2 \\
\hline hsa04728 & Dopaminergic synapse & $3 / 18$ & $132 / 8018$ & 0.009 & PPP1CA GSK3A GSK3B \\
\hline hsa04550 & $\begin{array}{l}\text { Signaling pathways regulating pluripotency of } \\
\text { stem cells }\end{array}$ & $3 / 18$ & $142 / 8018$ & 0.01 & STAT3 SMAD3 GSK3B \\
\hline hsa05168 & Herpes simplex virus 1 infection & $5 / 18$ & $491 / 8018$ & 0.01 & PPP1CA IRF3 BCL2L1 BCL2 IL6 \\
\hline hsa05160 & Hepatitis C & $3 / 18$ & $155 / 8018$ & 0.013 & STAT3 IRF3 GSK3B \\
\hline hsa04310 & Wnt signaling pathway & $3 / 18$ & $160 / 8018$ & 0.014 & JUN SMAD3 GSK3B \\
\hline hsa04672 & Intestinal immune network for IgA production & $2 / 18$ & $49 / 8018$ & 0.014 & TGFB1 IL6 \\
\hline hsa04340 & Hedgehog signaling pathway & $2 / 18$ & $50 / 8018$ & 0.014 & BCL2 GSK3B \\
\hline hsa05144 & Malaria & $2 / 18$ & $50 / 8018$ & 0.014 & TGFB1 IL6 \\
\hline hsa05014 & Amyotrophic lateral sclerosis (ALS) & $2 / 18$ & $57 / 8018$ & 0.017 & BCL2L1 BCL2 \\
\hline hsa05152 & Tuberculosis & $3 / 18$ & $180 / 8018$ & 0.017 & TGFB1 BCL2 IL6 \\
\hline
\end{tabular}




\begin{tabular}{|c|c|c|c|c|c|}
\hline ID & Description & GeneRatio & BgRatio & p.adjust & geneID \\
\hline hsa04062 & Chemokine signaling pathway & $3 / 18$ & $189 / 8018$ & 0.02 & STAT3 GSK3A GSK3B \\
\hline hsa04623 & Cytosolic DNA-sensing pathway & $2 / 18$ & $63 / 8018$ & 0.02 & IRF3 IL6 \\
\hline hsa05223 & Non-small cell lung cancer & $2 / 18$ & $66 / 8018$ & 0.022 & STAT5A STAT3 \\
\hline hsa05221 & Acute myeloid leukemia & $2 / 18$ & $67 / 8018$ & 0.022 & STAT5A STAT3 \\
\hline hsa05205 & Proteoglycans in cancer & $3 / 18$ & $204 / 8018$ & 0.022 & STAT3 PPP1CA TGFB1 \\
\hline hsa05031 & Amphetamine addiction & $2 / 18$ & $69 / 8018$ & 0.022 & JUN PPP1CA \\
\hline hsa05211 & Renal cell carcinoma & $2 / 18$ & $69 / 8018$ & 0.022 & JUN TGFB1 \\
\hline hsa04115 & p53 signaling pathway & $2 / 18$ & $72 / 8018$ & 0.024 & BCL2L1 BCL2 \\
\hline hsa05132 & Salmonella infection & $3 / 18$ & $214 / 8018$ & 0.024 & JUN BCL2 IL6 \\
\hline hsa01524 & Platinum drug resistance & $2 / 18$ & $73 / 8018$ & 0.024 & BCL2L1 BCL2 \\
\hline hsa05140 & Leishmaniasis & $2 / 18$ & $77 / 8018$ & 0.026 & JUN TGFB1 \\
\hline hsa04662 & B cell receptor signaling pathway & $2 / 18$ & $82 / 8018$ & 0.029 & JUN GSK3B \\
\hline hsa05235 & $\begin{array}{l}\text { PD-L1 expression and PD-1 checkpoint } \\
\text { pathway in cancer }\end{array}$ & $2 / 18$ & $89 / 8018$ & 0.033 & JUN STAT3 \\
\hline hsa05410 & Hypertrophic cardiomyopathy (HCM) & $2 / 18$ & $90 / 8018$ & 0.033 & TGFB1 IL6 \\
\hline hsa04658 & Th1 and Th2 cell differentiation & $2 / 18$ & $92 / 8018$ & 0.034 & STAT5A JUN \\
\hline hsa04350 & TGF-beta signaling pathway & $2 / 18$ & $94 / 8018$ & 0.035 & TGFB1 SMAD3 \\
\hline hsa05215 & Prostate cancer & $2 / 18$ & $97 / 8018$ & 0.037 & BCL2 GSK3B \\
\hline hsa01522 & Endocrine resistance & $2 / 18$ & $98 / 8018$ & 0.037 & JUN BCL2 \\
\hline hsa05146 & Amoebiasis & $2 / 18$ & $102 / 8018$ & 0.04 & TGFB1 IL6 \\
\hline hsa04625 & C-type lectin receptor signaling pathway & $2 / 18$ & $104 / 8018$ & 0.04 & JUN IL6 \\
\hline hsa04660 & $\mathrm{T}$ cell receptor signaling pathway & $2 / 18$ & $104 / 8018$ & 0.04 & JUN GSK3B \\
\hline hsa04928 & $\begin{array}{l}\text { Parathyroid hormone synthesis, secretion and } \\
\text { action }\end{array}$ & $2 / 18$ & $106 / 8018$ & 0.041 & BCL2 NACA \\
\hline hsa04668 & TNF signaling pathway & $2 / 18$ & $112 / 8018$ & 0.045 & JUN IL6 \\
\hline
\end{tabular}




\subsection{WIKI PATHWAY}

\section{BARPLOT}

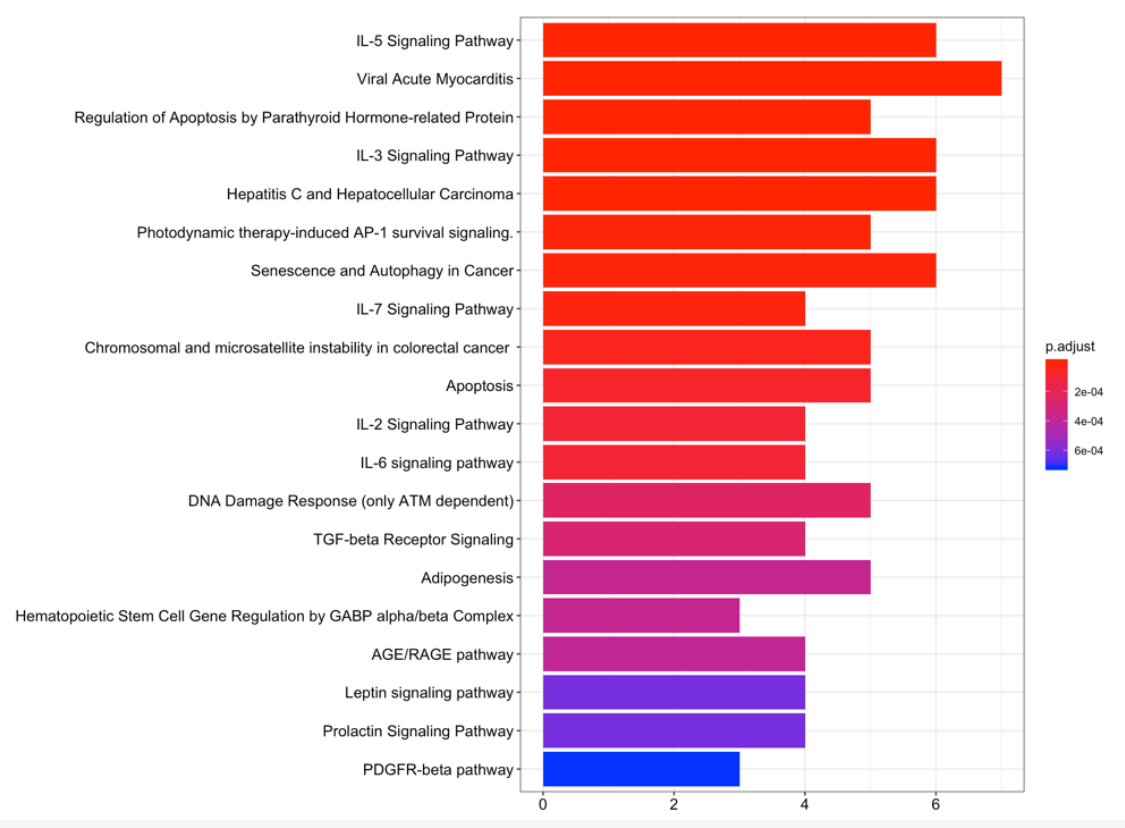

\section{NETPLOT}

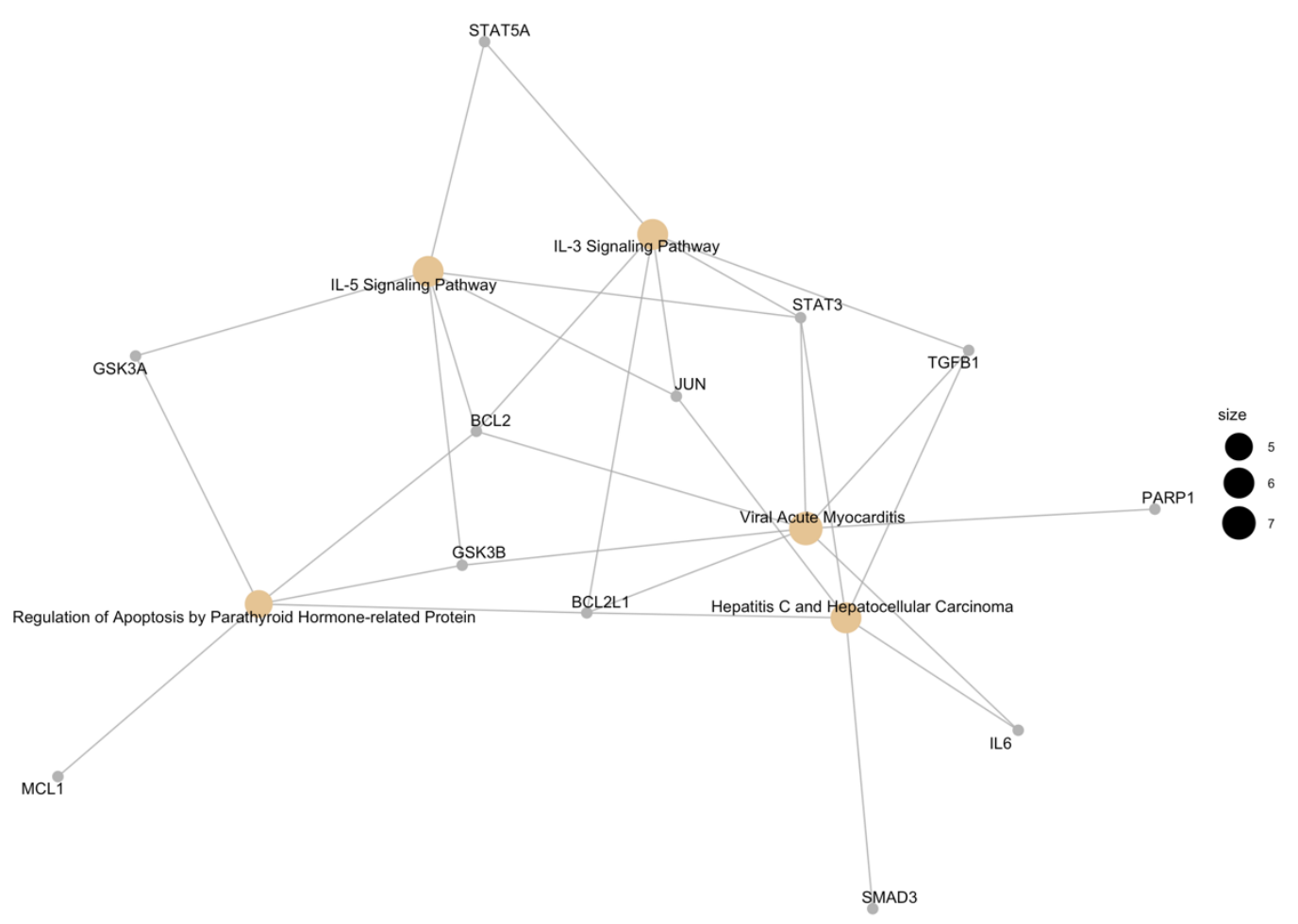




\begin{tabular}{|c|c|c|c|c|c|}
\hline ID & Description & GeneRatio & BgRatio & p.adjust & geneID \\
\hline WP127 & IL-5 Signaling Pathway & $6 / 19$ & $40 / 6249$ & 0 & $\begin{array}{l}\text { STAT5A JUN STAT3 } \\
\text { BCL2 GSK3A GSK3B }\end{array}$ \\
\hline WP4298 & Viral Acute Myocarditis & $7 / 19$ & $85 / 6249$ & 0 & $\begin{array}{c}\text { STAT3 TGFB1 BCL2L1 } \\
\text { BCL2 GSK3B PARP1 } \\
\text { IL6 }\end{array}$ \\
\hline WP3872 & $\begin{array}{c}\text { Regulation of Apoptosis by Parathyroid Hormone-related } \\
\text { Protein }\end{array}$ & $5 / 19$ & $22 / 6249$ & 0 & $\begin{array}{l}\text { BCL2L1 MCL1 BCL2 } \\
\text { GSK3A GSK3B }\end{array}$ \\
\hline WP286 & IL-3 Signaling Pathway & $6 / 19$ & $49 / 6249$ & 0 & $\begin{array}{l}\text { STAT5A JUN STAT3 } \\
\text { TGFB1 BCL2L1 BCL2 }\end{array}$ \\
\hline WP3646 & Hepatitis C and Hepatocellular Carcinoma & $6 / 19$ & $51 / 6249$ & 0 & $\begin{array}{l}\text { JUN STAT3 TGFB1 } \\
\text { SMAD3 BCL2L1 IL6 }\end{array}$ \\
\hline WP3611 & Photodynamic therapy-induced AP-1 survival signaling. & $5 / 19$ & $51 / 6249$ & 0 & $\begin{array}{l}\text { JUN BCL2L1 MCL1 } \\
\text { BCL2 IL6 }\end{array}$ \\
\hline WP615 & Senescence and Autophagy in Cancer & $6 / 19$ & $106 / 6249$ & 0 & $\begin{array}{l}\text { JUN TGFB1 SMAD3 } \\
\text { BCL2 GSK3B IL6 }\end{array}$ \\
\hline WP205 & IL-7 Signaling Pathway & $4 / 19$ & $25 / 6249$ & 0 & $\begin{array}{l}\text { STAT5A STAT3 } \\
\text { BCL2L1 GSK3B }\end{array}$ \\
\hline WP4216 & $\begin{array}{l}\text { Chromosomal and microsatellite instability in colorectal } \\
\text { cancer }\end{array}$ & $5 / 19$ & $74 / 6249$ & 0 & $\begin{array}{l}\text { JUN TGFB1 SMAD3 } \\
\text { BCL2 GSK3B }\end{array}$ \\
\hline WP254 & Apoptosis & $5 / 19$ & $86 / 6249$ & 0 & $\begin{array}{l}\text { JUN IRF3 BCL2L1 } \\
\text { MCL1 BCL2 }\end{array}$ \\
\hline WP49 & IL-2 Signaling Pathway & $4 / 19$ & $42 / 6249$ & 0 & $\begin{array}{l}\text { STAT5A JUN STAT3 } \\
\text { BCL2 }\end{array}$ \\
\hline WP364 & IL-6 signaling pathway & $4 / 19$ & $43 / 6249$ & 0 & $\begin{array}{l}\text { STAT3 BCL2L1 GSK3B } \\
\text { IL6 }\end{array}$ \\
\hline WP710 & DNA Damage Response (only ATM dependent) & $5 / 19$ & $115 / 6249$ & 0 & $\begin{array}{l}\text { JUN TGFB1 SMAD3 } \\
\text { BCL2 GSK3B }\end{array}$ \\
\hline WP560 & TGF-beta Receptor Signaling & $4 / 19$ & $58 / 6249$ & 0 & $\begin{array}{l}\text { JUN STAT3 TGFB1 } \\
\text { SMAD3 }\end{array}$ \\
\hline WP236 & Adipogenesis & $5 / 19$ & $131 / 6249$ & 0 & $\begin{array}{l}\text { STAT5A STAT3 TGFB1 } \\
\text { SMAD3 IL6 }\end{array}$ \\
\hline WP3657 & $\begin{array}{l}\text { Hematopoietic Stem Cell Gene Regulation by GABP } \\
\text { alpha/beta Complex }\end{array}$ & $3 / 19$ & $22 / 6249$ & 0 & BCL2L1 MCL1 BCL2 \\
\hline WP2324 & AGE/RAGE pathway & $4 / 19$ & $66 / 6249$ & 0 & $\begin{array}{l}\text { STAT5A JUN STAT3 } \\
\text { SMAD3 }\end{array}$ \\
\hline WP2034 & Leptin signaling pathway & $4 / 19$ & $76 / 6249$ & 0.001 & $\begin{array}{l}\text { STAT3 BCL2L1 GSK3A } \\
\text { GSK3B }\end{array}$ \\
\hline WP2037 & Prolactin Signaling Pathway & $4 / 19$ & $76 / 6249$ & 0.001 & $\begin{array}{l}\text { STAT5A JUN STAT3 } \\
\text { GSK3B }\end{array}$ \\
\hline WP3972 & PDGFR-beta pathway & $3 / 19$ & $29 / 6249$ & 0.001 & STAT5A JUN STAT3 \\
\hline WP4263 & Pancreatic adenocarcinoma pathway & $4 / 19$ & $89 / 6249$ & 0.001 & $\begin{array}{l}\text { STAT3 TGFB1 SMAD3 } \\
\text { BCL2L1 }\end{array}$ \\
\hline WP138 & Androgen receptor signaling pathway & $4 / 19$ & $91 / 6249$ & 0.001 & $\begin{array}{l}\text { JUN STAT3 SMAD3 } \\
\text { GSK3B }\end{array}$ \\
\hline
\end{tabular}




\begin{tabular}{|c|c|c|c|c|c|}
\hline ID & Description & GeneRatio & BgRatio & p.adjust & geneID \\
\hline WP2355 & Corticotropin-releasing hormone signaling pathway & $4 / 19$ & $93 / 6249$ & 0.001 & $\begin{array}{l}\text { TGFB1 BCL2 GSK3B } \\
\text { PARP1 }\end{array}$ \\
\hline WP1772 & Apoptosis Modulation and Signaling & $4 / 19$ & $94 / 6249$ & 0.001 & $\begin{array}{l}\text { JUN BCL2L1 MCL1 } \\
\text { BCL2 }\end{array}$ \\
\hline WP2036 & $\begin{array}{c}\text { TNF related weak inducer of apoptosis (TWEAK) } \\
\text { Signaling Pathway }\end{array}$ & $3 / 19$ & $42 / 6249$ & 0.002 & JUN GSK3B IL6 \\
\hline WP2332 & Interleukin-11 Signaling Pathway & $3 / 19$ & $44 / 6249$ & 0.002 & STAT3 TGFB1 BCL2 \\
\hline WP314 & $\begin{array}{c}\text { Fas Ligand (FasL) pathway and Stress induction of Heat } \\
\text { Shock Proteins (HSP) regulation }\end{array}$ & $3 / 19$ & $44 / 6249$ & 0.002 & JUN BCL2 PARP1 \\
\hline WP3594 & Circadian rythm related genes & $5 / 19$ & $207 / 6249$ & 0.002 & $\begin{array}{l}\text { JUN PPP1CA ATF5 } \\
\text { GSK3B IL6 }\end{array}$ \\
\hline WP2203 & $\begin{array}{l}\text { Thymic Stromal LymphoPoietin (TSLP) Signaling } \\
\text { Pathway }\end{array}$ & $3 / 19$ & $47 / 6249$ & 0.002 & STAT5A STAT3 IL6 \\
\hline WP179 & Cell Cycle & $4 / 19$ & $122 / 6249$ & 0.002 & $\begin{array}{l}\text { TGFB1 SMAD3 SKP2 } \\
\text { GSK3B }\end{array}$ \\
\hline WP673 & ErbB Signaling Pathway & $3 / 19$ & $54 / 6249$ & 0.003 & STAT5A JUN GSK3B \\
\hline WP1559 & TFs Regulate miRNAs related to cardiac hypertrophy & $2 / 19$ & $12 / 6249$ & 0.003 & STAT3 TGFB1 \\
\hline WP3888 & VEGFA-VEGFR2 Signaling Pathway & $5 / 19$ & $238 / 6249$ & 0.003 & $\begin{array}{l}\text { JUN STAT3 BCL2L1 } \\
\text { BCL2 GSK3B }\end{array}$ \\
\hline WP2881 & Estrogen Receptor Pathway & $2 / 19$ & $13 / 6249$ & 0.003 & JUN STAT3 \\
\hline WP304 & Kit receptor signaling pathway & $3 / 19$ & $60 / 6249$ & 0.004 & STAT5A STAT3 BCL2 \\
\hline WP2380 & $\begin{array}{l}\text { Brain-Derived Neurotrophic Factor (BDNF) signaling } \\
\text { pathway }\end{array}$ & $4 / 19$ & $144 / 6249$ & 0.004 & $\begin{array}{l}\text { STAT5A JUN STAT3 } \\
\text { GSK3B }\end{array}$ \\
\hline WP1984 & Integrated Breast Cancer Pathway & $4 / 19$ & $154 / 6249$ & 0.005 & PHB JUN BCL2 GSK3A \\
\hline WP3680 & $\begin{array}{c}\text { Association Between Physico-Chemical Features and } \\
\text { Toxicity Associated Pathways }\end{array}$ & $3 / 19$ & $68 / 6249$ & 0.005 & STAT5A JUN GSK3B \\
\hline WP3874 & Canonical and Non-Canonical TGF-B signaling & $2 / 19$ & $17 / 6249$ & 0.005 & TGFB1 SMAD3 \\
\hline WP3859 & $\begin{array}{c}\text { TGF-B Signaling in Thyroid Cells for Epithelial- } \\
\text { Mesenchymal Transition }\end{array}$ & $2 / 19$ & $18 / 6249$ & 0.006 & TGFB1 SMAD3 \\
\hline WP22 & IL-9 Signaling Pathway & $2 / 19$ & $19 / 6249$ & 0.006 & STAT5A STAT3 \\
\hline WP3287 & Overview of nanoparticle effects & $2 / 19$ & $19 / 6249$ & 0.006 & BCL2 IL6 \\
\hline WP2507 & Nanomaterial induced apoptosis & $2 / 19$ & $21 / 6249$ & 0.007 & BCL2L1 MCL1 \\
\hline WP712 & Estrogen signaling pathway & $2 / 19$ & $23 / 6249$ & 0.008 & JUN BCL2 \\
\hline WP69 & T-Cell antigen Receptor (TCR) Signaling Pathway & $3 / 19$ & $91 / 6249$ & 0.009 & JUN TGFB1 IL6 \\
\hline WP1528 & Physiological and Pathological Hypertrophy of the Heart & $2 / 19$ & $25 / 6249$ & 0.009 & JUN STAT3 \\
\hline WP231 & TNF alpha Signaling Pathway & $3 / 19$ & $94 / 6249$ & 0.01 & JUN BCL2L1 IL6 \\
\hline WP581 & EPO Receptor Signaling & $2 / 19$ & $26 / 6249$ & 0.01 & STAT5A STAT3 \\
\hline
\end{tabular}




\begin{tabular}{|c|c|c|c|c|c|}
\hline ID & Description & GeneRatio & BgRatio & p.adjust & geneID \\
\hline WP306 & Focal Adhesion & $4 / 19$ & $202 / 6249$ & 0.01 & $\begin{array}{l}\text { JUN PPP1CA BCL2 } \\
\text { GSK3B }\end{array}$ \\
\hline WP530 & Cytokines and Inflammatory Response & $2 / 19$ & $27 / 6249$ & 0.01 & TGFB1 IL6 \\
\hline WP23 & B Cell Receptor Signaling Pathway & $3 / 19$ & $98 / 6249$ & 0.01 & JUN GSK3A GSK3B \\
\hline WP4172 & PI3K-Akt Signaling Pathway & $5 / 19$ & $345 / 6249$ & 0.01 & $\begin{array}{l}\text { BCL2L1 MCL1 BCL2 } \\
\text { GSK3B IL6 }\end{array}$ \\
\hline WP4300 & Extracellular vesicles in the crosstalk of cardiac cells & $2 / 19$ & $28 / 6249$ & 0.01 & STAT3 IL6 \\
\hline WP1544 & MicroRNAs in cardiomyocyte hypertrophy & $3 / 19$ & $102 / 6249$ & 0.01 & STAT3 TGFB1 GSK3B \\
\hline WP2583 & T-Cell Receptor and Co-stimulatory Signaling & $2 / 19$ & $29 / 6249$ & 0.01 & GSK3A GSK3B \\
\hline WP75 & Toll-like Receptor Signaling Pathway & $3 / 19$ & $103 / 6249$ & 0.01 & JUN IRF3 IL6 \\
\hline WP2855 & Dopaminergic Neurogenesis & $2 / 19$ & $30 / 6249$ & 0.01 & STAT3 TGFB1 \\
\hline WP2870 & Extracellular vesicle-mediated signaling in recipient cells & $2 / 19$ & $30 / 6249$ & 0.01 & TGFB1 SMAD3 \\
\hline WP3844 & $\begin{array}{c}\text { PI3K-AKT-mTOR signaling pathway and therapeutic } \\
\text { opportunities }\end{array}$ & $2 / 19$ & $30 / 6249$ & 0.01 & TFEB GSK3B \\
\hline WP3851 & TLR4 Signaling and Tolerance & $2 / 19$ & $30 / 6249$ & 0.01 & IRF3 IL6 \\
\hline WP2112 & IL17 signaling pathway & $2 / 19$ & $31 / 6249$ & 0.011 & STAT3 GSK3B \\
\hline WP3850 & $\begin{array}{l}\text { Factors and pathways affecting insulin-like growth factor } \\
\text { (IGF1)-Akt signaling }\end{array}$ & $2 / 19$ & $31 / 6249$ & 0.011 & SMAD3 GSK3B \\
\hline WP4149 & White fat cell differentiation & $2 / 19$ & $33 / 6249$ & 0.012 & STAT5A IRF3 \\
\hline WP313 & Signaling of Hepatocyte Growth Factor Receptor & $2 / 19$ & $34 / 6249$ & 0.013 & JUN STAT3 \\
\hline WP4241 & Type 2 papillary renal cell carcinoma & $2 / 19$ & $36 / 6249$ & 0.014 & TFEB TGFB1 \\
\hline WP3931 & ESC Pluripotency Pathways & $3 / 19$ & $119 / 6249$ & 0.014 & JUN STAT3 GSK3B \\
\hline WP3614 & Photodynamic therapy-induced HIF-1 survival signaling & $2 / 19$ & $37 / 6249$ & 0.014 & BCL2L1 MCL1 \\
\hline WP3995 & Prion disease pathway & $2 / 19$ & $37 / 6249$ & 0.014 & STAT3 BCL2 \\
\hline WP2447 & Amyotrophic lateral sclerosis (ALS) & $2 / 19$ & $38 / 6249$ & 0.014 & BCL2L1 BCL2 \\
\hline WP4136 & Fibrin Complement Receptor 3 Signaling Pathway & $2 / 19$ & $38 / 6249$ & 0.014 & IRF3 IL6 \\
\hline WP2526 & PDGF Pathway & $2 / 19$ & $39 / 6249$ & 0.015 & JUN STAT3 \\
\hline WP500 & Glycogen Metabolism & $2 / 19$ & $40 / 6249$ & 0.015 & GSK3A GSK3B \\
\hline WP366 & TGF-beta Signaling Pathway & $3 / 19$ & $133 / 6249$ & 0.017 & JUN TGFB1 SMAD3 \\
\hline WP1971 & Integrated Cancer Pathway & $2 / 19$ & $46 / 6249$ & 0.02 & SMAD3 BCL2 \\
\hline WP1449 & Regulation of toll-like receptor signaling pathway & $3 / 19$ & $145 / 6249$ & 0.02 & JUN IRF3 IL6 \\
\hline WP2848 & Differentiation Pathway & $2 / 19$ & $48 / 6249$ & 0.02 & TGFB1 IL6 \\
\hline WP2873 & Aryl Hydrocarbon Receptor Pathway & $2 / 19$ & $48 / 6249$ & 0.02 & JUN TGFB1 \\
\hline
\end{tabular}




\begin{tabular}{|c|c|c|c|c|c|}
\hline ID & Description & GeneRatio & BgRatio & p.adjust & geneID \\
\hline WP2038 & Regulation of Microtubule Cytoskeleton & $2 / 19$ & $49 / 6249$ & 0.021 & STAT3 GSK3B \\
\hline WP2406 & Cardiac Progenitor Differentiation & $2 / 19$ & $53 / 6249$ & 0.024 & TGFB1 GSK3B \\
\hline WP363 & Wnt Signaling Pathway & $2 / 19$ & $53 / 6249$ & 0.024 & GSK3A GSK3B \\
\hline WP4262 & Breast cancer pathway & $3 / 19$ & $157 / 6249$ & 0.024 & JUN GSK3B PARP1 \\
\hline WP3286 & Copper homeostasis & $2 / 19$ & $54 / 6249$ & 0.024 & JUN GSK3B \\
\hline WP289 & Myometrial Relaxation and Contraction Pathways & $3 / 19$ & $158 / 6249$ & 0.024 & JUN ATF5 IL6 \\
\hline WP395 & IL-4 Signaling Pathway & $2 / 19$ & $55 / 6249$ & 0.024 & STAT5A STAT3 \\
\hline WP585 & Interferon type I signaling pathways & $2 / 19$ & $55 / 6249$ & 0.024 & STAT5A STAT3 \\
\hline WP481 & Insulin Signaling & $3 / 19$ & $161 / 6249$ & 0.024 & JUN GSK3A GSK3B \\
\hline WP4239 & Epithelial to mesenchymal transition in colorectal cancer & $3 / 19$ & $164 / 6249$ & 0.025 & TGFB1 SMAD3 GSK3B \\
\hline WP437 & EGF/EGFR Signaling Pathway & $3 / 19$ & $164 / 6249$ & 0.025 & STAT5A JUN STAT3 \\
\hline WP2795 & Cardiac Hypertrophic Response & $2 / 19$ & $57 / 6249$ & 0.025 & TGFB1 GSK3B \\
\hline WP3981 & miRNA regulation of prostate cancer signaling pathways & $2 / 19$ & $59 / 6249$ & 0.026 & BCL2 GSK3B \\
\hline WP4205 & MET in type 1 papillary renal cell carcinoma & $2 / 19$ & $59 / 6249$ & 0.026 & JUN STAT3 \\
\hline WP3863 & $\begin{array}{l}\text { T-Cell antigen Receptor (TCR) pathway during } \\
\text { Staphylococcus aureus infection }\end{array}$ & $2 / 19$ & $62 / 6249$ & 0.028 & JUN GSK3B \\
\hline WP2849 & Hematopoietic Stem Cell Differentiation & $2 / 19$ & $63 / 6249$ & 0.028 & STAT5A IL6 \\
\hline WP3624 & Lung fibrosis & $2 / 19$ & $63 / 6249$ & 0.028 & TGFB1 IL6 \\
\hline WP61 & Notch Signaling Pathway & $2 / 19$ & $63 / 6249$ & 0.028 & STAT3 GSK3B \\
\hline WP4255 & Non-small cell lung cancer & $2 / 19$ & $66 / 6249$ & 0.03 & STAT5A STAT3 \\
\hline WP2032 & $\begin{array}{l}\text { Human Thyroid Stimulating Hormone (TSH) signaling } \\
\text { pathway }\end{array}$ & $2 / 19$ & $68 / 6249$ & 0.032 & JUN STAT3 \\
\hline WP3303 & RAC1/PAK1/p38/MMP2 Pathway & $2 / 19$ & $69 / 6249$ & 0.032 & STAT5A STAT3 \\
\hline WP1982 & $\begin{array}{c}\text { Sterol Regulatory Element-Binding Proteins (SREBP) } \\
\text { signalling }\end{array}$ & $2 / 19$ & $73 / 6249$ & 0.035 & KPNB1 GSK3A \\
\hline WP4018 & Pathways in clear cell renal cell carcinoma & $2 / 19$ & $87 / 6249$ & 0.049 & STAT3 TGFB1 \\
\hline WP2840 & $\begin{array}{l}\text { Hair Follicle Development: Cytodifferentiation (Part } 3 \text { of } \\
\text { 3) }\end{array}$ & $2 / 19$ & $88 / 6249$ & 0.049 & JUN TGFB1 \\
\hline
\end{tabular}




\section{CLUSTER ANALYSIS}

\section{CLUSTER COMPARISON}

GO

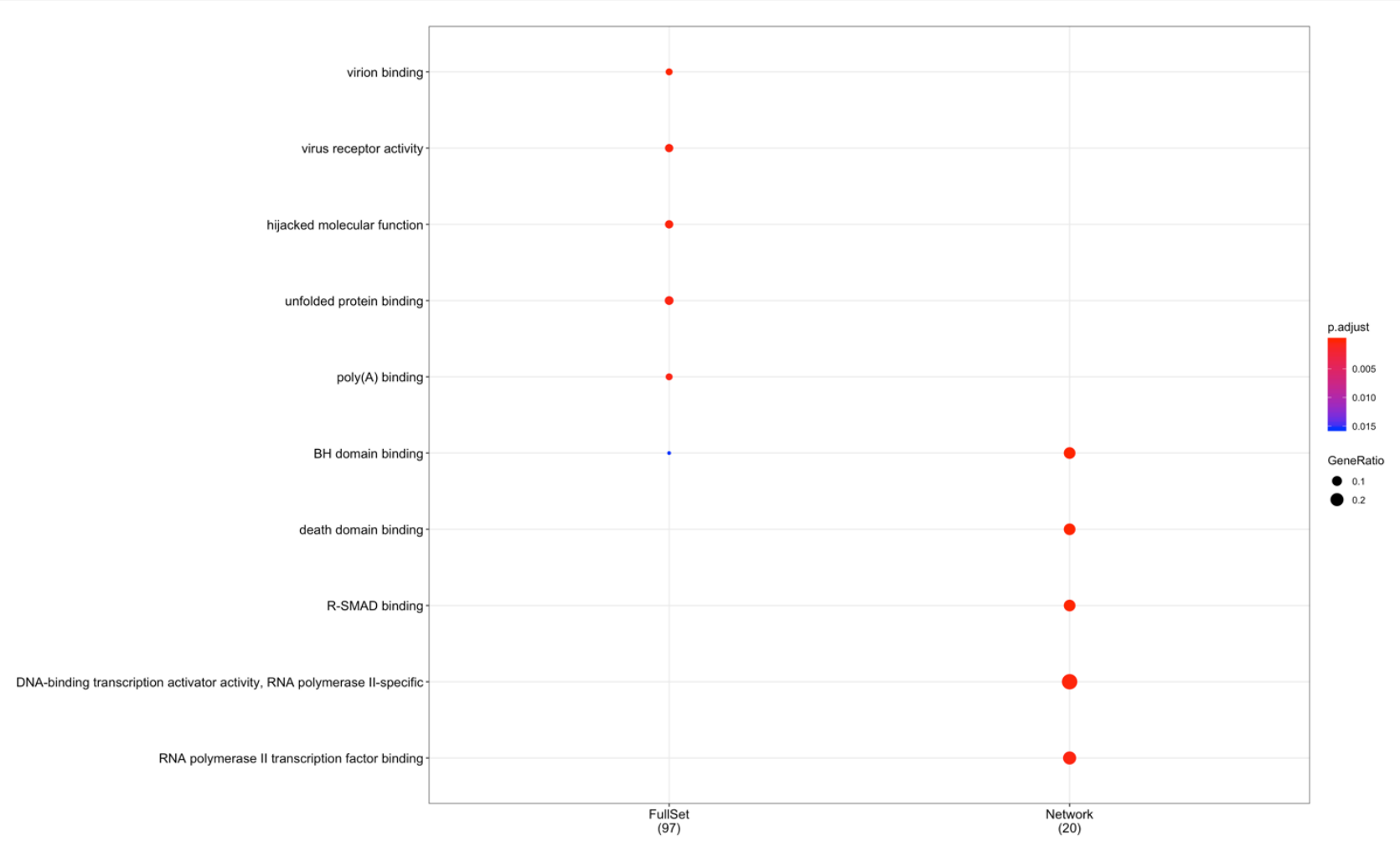




\section{KEGG PATHWAY}

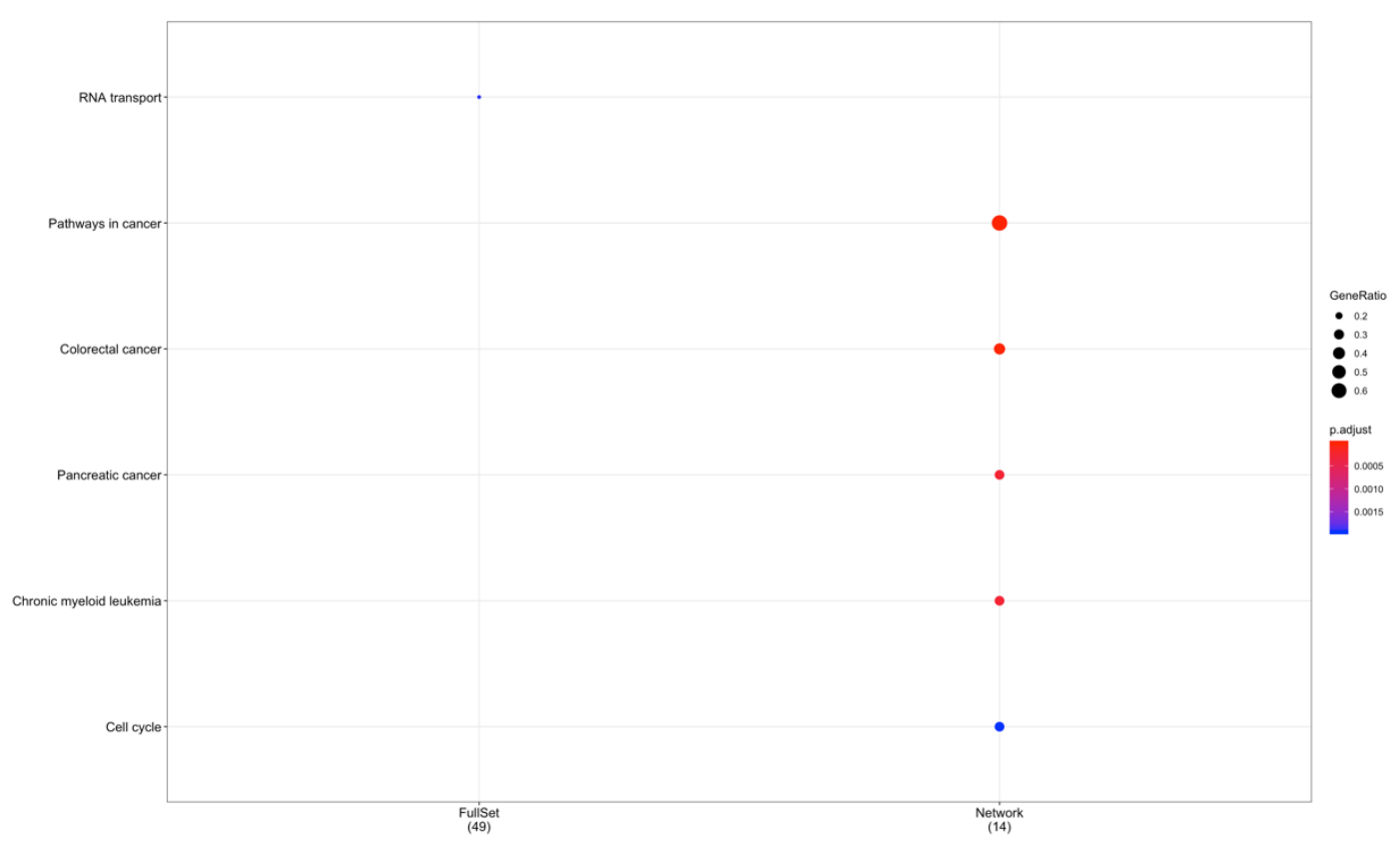

GO.LOCATION

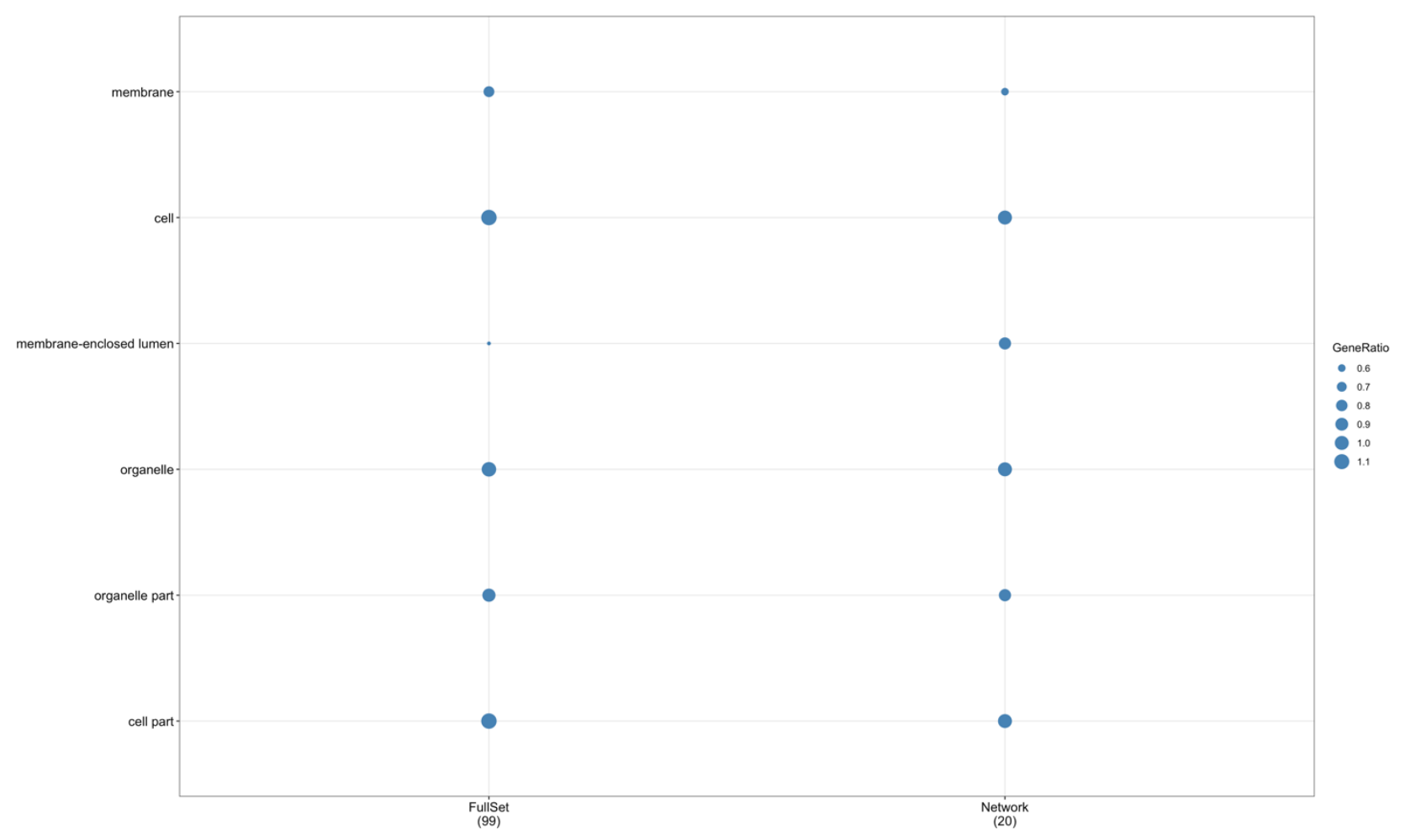




\subsection{GO}

\begin{tabular}{|c|c|c|c|c|c|c|c|}
\hline Cluster & group & ID & Description & GeneRatio & BgRatio & p.adjust & geneID \\
\hline FullSet & FullSet & GO:0046790 & virion binding & $4 / 97$ & $10 / 17632$ & 0 & $\begin{array}{c}3083510332 \\
5478302\end{array}$ \\
\hline FullSet & FullSet & GO:0001618 & virus receptor activity & $6 / 97$ & $74 / 17632$ & 0 & $\begin{array}{c}59272339390 \\
3083510332 \\
2901803\end{array}$ \\
\hline FullSet & FullSet & GO:0104005 & $\begin{array}{l}\text { hijacked molecular } \\
\text { function }\end{array}$ & $6 / 97$ & $74 / 17632$ & 0 & $\begin{array}{c}59272339390 \\
3083510332 \\
2901803\end{array}$ \\
\hline FullSet & FullSet & GO:0051082 & unfolded protein binding & $7 / 97$ & $127 / 17632$ & 0 & $\begin{array}{c}54783337 \\
33293313 \\
936010465 \\
5204\end{array}$ \\
\hline FullSet & FullSet & GO:0008143 & poly(A) binding & $4 / 97$ & $24 / 17632$ & 0.001 & $\begin{array}{c}1049226986 \\
87611653\end{array}$ \\
\hline FullSet & FullSet & GO:0003727 & $\begin{array}{l}\text { single-stranded RNA } \\
\text { binding }\end{array}$ & $6 / 97$ & $90 / 17632$ & 0.001 & $\begin{array}{c}317810492 \\
572526986 \\
8761 \quad 1653\end{array}$ \\
\hline FullSet & FullSet & GO:0070717 & poly-purine tract binding & $4 / 97$ & $30 / 17632$ & 0.001 & $\begin{array}{c}1049226986 \\
87611653\end{array}$ \\
\hline FullSet & FullSet & GO:0033218 & amide binding & $10 / 97$ & $348 / 17632$ & 0.001 & $\begin{array}{c}9145230835 \\
103323838 \\
54782903329 \\
67299360 \\
10465\end{array}$ \\
\hline FullSet & FullSet & GO:0098505 & $\begin{array}{l}\text { G-rich strand telomeric } \\
\text { DNA binding }\end{array}$ & $3 / 97$ & $11 / 17632$ & 0.001 & $\begin{array}{l}31783181 \\
7013\end{array}$ \\
\hline FullSet & FullSet & GO:0042277 & peptide binding & 9/97 & $285 / 17632$ & 0.001 & $\begin{array}{c}3083510332 \\
38385478290 \\
33296729 \\
936010465\end{array}$ \\
\hline FullSet & FullSet & GO:0043047 & $\begin{array}{l}\text { single-stranded } \\
\text { telomeric DNA binding }\end{array}$ & $3 / 97$ & $13 / 17632$ & 0.001 & $\begin{array}{l}31783181 \\
\quad 7013\end{array}$ \\
\hline FullSet & FullSet & GO:0003755 & $\begin{array}{l}\text { peptidyl-prolyl cis-trans } \\
\text { isomerase activity }\end{array}$ & $4 / 97$ & $37 / 17632$ & 0.001 & $\begin{array}{c}54782280 \\
936010465\end{array}$ \\
\hline FullSet & FullSet & GO:0042162 & telomeric DNA binding & $4 / 97$ & $38 / 17632$ & 0.001 & $\begin{array}{l}31783181 \\
46917013\end{array}$ \\
\hline FullSet & FullSet & GO:0098847 & $\begin{array}{l}\text { sequence-specific single } \\
\text { stranded DNA binding }\end{array}$ & $3 / 97$ & $15 / 17632$ & 0.001 & $\begin{array}{l}31783181 \\
7013\end{array}$ \\
\hline FullSet & FullSet & GO:0045296 & cadherin binding & 9/97 & $323 / 17632$ & 0.001 & $\begin{array}{c}1134410652 \\
68113023337 \\
3646128866 \\
5142925978\end{array}$ \\
\hline FullSet & FullSet & GO:0016859 & $\begin{array}{l}\text { cis-trans isomerase } \\
\text { activity }\end{array}$ & $4 / 97$ & $41 / 17632$ & 0.001 & $\begin{array}{c}54782280 \\
936010465\end{array}$ \\
\hline
\end{tabular}




\begin{tabular}{|c|c|c|c|c|c|c|c|}
\hline Cluster & group & ID & Description & GeneRatio & BgRatio & p.adjust & geneID \\
\hline FullSet & FullSet & GO:0016018 & cyclosporin A binding & $3 / 97$ & $19 / 17632$ & 0.003 & $\begin{array}{l}54789360 \\
10465\end{array}$ \\
\hline FullSet & FullSet & GO:0140142 & $\begin{array}{l}\text { nucleocytoplasmic } \\
\text { carrier activity }\end{array}$ & $3 / 97$ & $19 / 17632$ & 0.003 & $\begin{array}{c}75143838 \\
3840\end{array}$ \\
\hline FullSet & FullSet & GO:0003729 & mRNA binding & $10 / 97$ & $463 / 17632$ & 0.004 & $\begin{array}{c}31783181 \\
22098823521 \\
269868761 \\
101469908 \\
46911655\end{array}$ \\
\hline FullSet & FullSet & GO:0050839 & $\begin{array}{l}\text { cell adhesion molecule } \\
\text { binding }\end{array}$ & $10 / 97$ & $486 / 17632$ & 0.005 & $\begin{array}{c}1134410652 \\
6811928302 \\
33373646 \\
12886651429 \\
25978\end{array}$ \\
\hline FullSet & FullSet & GO:0036002 & pre-mRNA binding & $4 / 97$ & $62 / 17632$ & 0.005 & $\begin{array}{l}31785725 \\
31811655\end{array}$ \\
\hline FullSet & FullSet & GO:0005484 & SNAP receptor activity & $3 / 97$ & $30 / 17632$ & 0.008 & $\begin{array}{c}106526811 \\
116841\end{array}$ \\
\hline FullSet & FullSet & GO:0008187 & $\begin{array}{l}\text { poly-pyrimidine tract } \\
\text { binding }\end{array}$ & $3 / 97$ & $33 / 17632$ & 0.01 & $\begin{array}{l}572526986 \\
8761\end{array}$ \\
\hline FullSet & FullSet & GO:0003730 & mRNA 3'-UTR binding & $4 / 97$ & $80 / 17632$ & 0.013 & $\begin{array}{l}318126986 \\
87611655\end{array}$ \\
\hline FullSet & FullSet & GO:0051400 & BH domain binding & $2 / 97$ & $10 / 17632$ & 0.015 & 599597 \\
\hline FullSet & FullSet & GO:0061608 & $\begin{array}{l}\text { nuclear import signal } \\
\text { receptor activity }\end{array}$ & $2 / 97$ & $10 / 17632$ & 0.015 & 38383840 \\
\hline FullSet & FullSet & GO:0044389 & $\begin{array}{l}\text { ubiquitin-like protein } \\
\text { ligase binding }\end{array}$ & $7 / 97$ & $301 / 17632$ & 0.016 & $\begin{array}{c}33293313 \\
570751429 \\
91467917 \\
11200\end{array}$ \\
\hline FullSet & FullSet & GO:0008135 & $\begin{array}{l}\text { translation factor } \\
\text { activity, RNA binding }\end{array}$ & $4 / 97$ & $89 / 17632$ & 0.016 & $\begin{array}{l}36468668 \\
86651915\end{array}$ \\
\hline FullSet & FullSet & GO:0140104 & $\begin{array}{l}\text { molecular carrier } \\
\text { activity }\end{array}$ & $3 / 97$ & $43 / 17632$ & 0.018 & $\begin{array}{l}75143838 \\
3840\end{array}$ \\
\hline FullSet & FullSet & GO:0008494 & $\begin{array}{c}\text { translation activator } \\
\text { activity }\end{array}$ & $2 / 97$ & $13 / 17632$ & 0.023 & 2698657409 \\
\hline FullSet & FullSet & GO:0003724 & RNA helicase activity & $3 / 97$ & $48 / 17632$ & 0.023 & $\begin{array}{c}101461653 \\
1655\end{array}$ \\
\hline FullSet & FullSet & GO:0003697 & $\begin{array}{l}\text { single-stranded DNA } \\
\text { binding }\end{array}$ & $4 / 97$ & $105 / 17632$ & 0.026 & $\begin{array}{l}31783181 \\
33297013\end{array}$ \\
\hline FullSet & FullSet & GO:0003743 & $\begin{array}{l}\text { translation initiation } \\
\text { factor activity }\end{array}$ & $3 / 97$ & $51 / 17632$ & 0.026 & $\begin{array}{l}36468668 \\
\quad 8665\end{array}$ \\
\hline FullSet & FullSet & GO:0016887 & ATPase activity & $8 / 97$ & $445 / 17632$ & 0.028 & $\begin{array}{c}57013329 \\
33133832 \\
101461653 \\
16559550\end{array}$ \\
\hline FullSet & FullSet & GO:0042623 & $\begin{array}{l}\text { ATPase activity, } \\
\text { coupled }\end{array}$ & $7 / 97$ & $357 / 17632$ & 0.031 & $\begin{array}{c}57013313 \\
383210146 \\
16531655 \\
9550\end{array}$ \\
\hline
\end{tabular}




\begin{tabular}{|c|c|c|c|c|c|c|c|}
\hline Cluster & group & ID & Description & GeneRatio & BgRatio & p.adjust & geneID \\
\hline FullSet & FullSet & GO:0048306 & $\begin{array}{l}\text { calcium-dependent } \\
\text { protein binding }\end{array}$ & $3 / 97$ & $62 / 17632$ & 0.041 & $\begin{array}{c}10332302 \\
1655\end{array}$ \\
\hline FullSet & FullSet & GO:0031625 & $\begin{array}{l}\text { ubiquitin protein ligase } \\
\text { binding }\end{array}$ & $6 / 97$ & $286 / 17632$ & 0.041 & $\begin{array}{c}33293313 \\
570751429 \\
791711200\end{array}$ \\
\hline FullSet & FullSet & GO:0002020 & protease binding & $4 / 97$ & $125 / 17632$ & 0.041 & $\begin{array}{c}18033023329 \\
7917\end{array}$ \\
\hline FullSet & FullSet & GO:0043021 & $\begin{array}{l}\text { ribonucleoprotein } \\
\text { complex binding }\end{array}$ & $4 / 97$ & $128 / 17632$ & 0.043 & $\begin{array}{l}672910465 \\
79171655\end{array}$ \\
\hline FullSet & FullSet & GO:0005537 & mannose binding & $2 / 97$ & $22 / 17632$ & 0.049 & 3083510332 \\
\hline Network & Network & GO:0051400 & $\mathrm{BH}$ domain binding & $3 / 20$ & $10 / 17632$ & 0 & 5984170596 \\
\hline Network & Network & GO:0070513 & death domain binding & $3 / 20$ & $10 / 17632$ & 0 & 5984170596 \\
\hline Network & Network & GO:0070412 & R-SMAD binding & $3 / 20$ & $23 / 17632$ & 0 & 37254088142 \\
\hline Network & Network & GO:0001228 & $\begin{array}{c}\text { DNA-binding } \\
\text { transcription activator } \\
\text { activity, RNA } \\
\text { polymerase II-specific }\end{array}$ & $6 / 20$ & $449 / 17632$ & 0 & $\begin{array}{c}37256774 \\
408822809 \\
3661142\end{array}$ \\
\hline Network & Network & GO:0001085 & $\begin{array}{l}\text { RNA polymerase II } \\
\text { transcription factor } \\
\text { binding }\end{array}$ & $4 / 20$ & $140 / 17632$ & 0 & $\begin{array}{l}37256774 \\
40882932\end{array}$ \\
\hline Network & Network & GO:0019902 & phosphatase binding & $4 / 20$ & $178 / 17632$ & 0.001 & $\begin{array}{c}67745499 \\
4088596\end{array}$ \\
\hline Network & Network & GO:0033613 & $\begin{array}{l}\text { activating transcription } \\
\text { factor binding }\end{array}$ & $3 / 20$ & $76 / 17632$ & 0.001 & $\begin{array}{c}37254088 \\
4869\end{array}$ \\
\hline Network & Network & GO:0034236 & $\begin{array}{c}\text { protein kinase A } \\
\text { catalytic subunit binding }\end{array}$ & $2 / 20$ & $13 / 17632$ & 0.001 & 29312932 \\
\hline Network & Network & GO:0046332 & SMAD binding & $3 / 20$ & $80 / 17632$ & 0.001 & 37254088142 \\
\hline Network & Network & GO:0035259 & $\begin{array}{l}\text { glucocorticoid receptor } \\
\text { binding }\end{array}$ & $2 / 20$ & $14 / 17632$ & 0.001 & 67744088 \\
\hline Network & Network & GO:0050321 & $\begin{array}{l}\text { tau-protein kinase } \\
\text { activity }\end{array}$ & $2 / 20$ & $15 / 17632$ & 0.001 & 29312932 \\
\hline Network & Network & GO:0035258 & $\begin{array}{l}\text { steroid hormone } \\
\text { receptor binding }\end{array}$ & $3 / 20$ & $90 / 17632$ & 0.001 & 67744088142 \\
\hline Network & Network & GO:0031625 & $\begin{array}{l}\text { ubiquitin protein ligase } \\
\text { binding }\end{array}$ & $4 / 20$ & $286 / 17632$ & 0.003 & $\begin{array}{c}37254088596 \\
2932\end{array}$ \\
\hline Network & Network & GO:0044389 & $\begin{array}{l}\text { ubiquitin-like protein } \\
\text { ligase binding }\end{array}$ & $4 / 20$ & $301 / 17632$ & 0.003 & $\begin{array}{c}37254088596 \\
2932\end{array}$ \\
\hline Network & Network & GO:0019903 & $\begin{array}{l}\text { protein phosphatase } \\
\text { binding }\end{array}$ & $3 / 20$ & $133 / 17632$ & 0.003 & 67745499596 \\
\hline Network & Network & GO:0051059 & NF-kappaB binding & $2 / 20$ & $28 / 17632$ & 0.003 & 29324869 \\
\hline Network & Network & GO:0035257 & $\begin{array}{l}\text { nuclear hormone } \\
\text { receptor binding }\end{array}$ & $3 / 20$ & $152 / 17632$ & 0.005 & 67744088142 \\
\hline
\end{tabular}




\begin{tabular}{|c|c|c|c|c|c|c|c|}
\hline Cluster & group & ID & Description & GeneRatio & BgRatio & p.adjust & geneID \\
\hline Network & Network & GO:0001102 & $\begin{array}{l}\text { RNA polymerase II } \\
\text { activating transcription } \\
\text { factor binding }\end{array}$ & $2 / 20$ & $44 / 17632$ & 0.007 & 37254088 \\
\hline Network & Network & GO:0051427 & $\begin{array}{l}\text { hormone receptor } \\
\text { binding }\end{array}$ & $3 / 20$ & $185 / 17632$ & 0.007 & 67744088142 \\
\hline Network & Network & GO:0051018 & protein kinase A binding & $2 / 20$ & $48 / 17632$ & 0.008 & 29312932 \\
\hline Network & Network & GO:0005160 & $\begin{array}{l}\text { transforming growth } \\
\text { factor beta receptor } \\
\text { binding }\end{array}$ & $2 / 20$ & $51 / 17632$ & 0.009 & 70404088 \\
\hline Network & Network & GO:0005126 & $\begin{array}{l}\text { cytokine receptor } \\
\text { binding }\end{array}$ & $3 / 20$ & $281 / 17632$ & 0.02 & $\begin{array}{c}67747040 \\
4088\end{array}$ \\
\hline Network & Network & GO:0008013 & beta-catenin binding & $2 / 20$ & $82 / 17632$ & 0.02 & 40882932 \\
\hline Network & Network & GO:0001077 & $\begin{array}{l}\text { proximal promoter } \\
\text { DNA-binding } \\
\text { transcription activator } \\
\text { activity, RNA } \\
\text { polymerase II-specific }\end{array}$ & $3 / 20$ & $298 / 17632$ & 0.022 & $\begin{array}{c}37256774 \\
3661\end{array}$ \\
\hline Network & Network & GO:0008565 & $\begin{array}{l}\text { protein transporter } \\
\text { activity }\end{array}$ & $2 / 20$ & $88 / 17632$ & 0.022 & 38374170 \\
\hline Network & Network & GO:0047485 & $\begin{array}{l}\text { protein N-terminus } \\
\text { binding }\end{array}$ & $2 / 20$ & $107 / 17632$ & 0.03 & 7040142 \\
\hline Network & Network & GO:0042826 & $\begin{array}{l}\text { histone deacetylase } \\
\text { binding }\end{array}$ & $2 / 20$ & $112 / 17632$ & 0.032 & 5245142 \\
\hline Network & Network & GO:0031490 & chromatin DNA binding & $2 / 20$ & $119 / 17632$ & 0.035 & 67744088 \\
\hline Network & Network & GO:0002020 & protease binding & $2 / 20$ & $125 / 17632$ & 0.037 & 5962932 \\
\hline Network & Network & GO:0043021 & $\begin{array}{l}\text { ribonucleoprotein } \\
\text { complex binding }\end{array}$ & $2 / 20$ & $128 / 17632$ & 0.037 & 54994869 \\
\hline Network & Network & GO:0035326 & enhancer binding & $2 / 20$ & $133 / 17632$ & 0.039 & 37254088 \\
\hline Network & Network & GO:0005072 & $\begin{array}{l}\text { transforming growth } \\
\text { factor beta receptor, } \\
\text { cytoplasmic mediator } \\
\text { activity }\end{array}$ & $1 / 20$ & $10 / 17632$ & 0.042 & 4088 \\
\hline Network & Network & GO:0030957 & Tat protein binding & $1 / 20$ & $10 / 17632$ & 0.042 & 4869 \\
\hline Network & Network & GO:0034713 & $\begin{array}{l}\text { type I transforming } \\
\text { growth factor beta } \\
\text { receptor binding }\end{array}$ & $1 / 20$ & $11 / 17632$ & 0.045 & 7040 \\
\hline Network & Network & GO:0000982 & $\begin{array}{l}\text { transcription factor } \\
\text { activity, RNA } \\
\text { polymerase II proximal } \\
\text { promoter sequence- } \\
\text { specific DNA binding }\end{array}$ & $3 / 20$ & $447 / 17632$ & 0.045 & $\begin{array}{c}37256774 \\
3661\end{array}$ \\
\hline Network & Network & GO:0034452 & dynactin binding & $1 / 20$ & $12 / 17632$ & 0.045 & 2932 \\
\hline Network & Network & GO:0070410 & co-SMAD binding & $1 / 20$ & $12 / 17632$ & 0.045 & 4088 \\
\hline Network & Network & GO:0043023 & $\begin{array}{l}\text { ribosomal large subunit } \\
\text { binding }\end{array}$ & $1 / 20$ & $13 / 17632$ & 0.047 & 4869 \\
\hline
\end{tabular}




\begin{tabular}{|c|c|c|c|c|c|c|}
\hline Cluster & group & ID & Description & GeneRatio & BgRatio & p.adjust \\
\hline Network & Network & GO:0001846 & opsonin binding & $14 / 17632$ \\
\hline Network & Network & GO:0035497 & cAMP response element \\
& & & binding & $1 / 20$ & $14 / 17632$ \\
\hline
\end{tabular}

\subsection{KEGG}

\begin{tabular}{|c|c|c|c|c|c|c|c|}
\hline Cluster & group & ID & Description & GeneRatio & BgRatio & p.adjust & geneID \\
\hline FullSet & FullSet & hsa03013 & RNA transport & $8 / 49$ & $152 / 5894$ & 0.002 & $\begin{array}{c}75143646 \\
86688665 \\
269868761 \\
19157329\end{array}$ \\
\hline Network & Network & hsa05200 & Pathways in cancer & $9 / 14$ & $327 / 5894$ & 0 & $\begin{array}{c}67763725 \\
67747040 \\
4088598596 \\
65022932\end{array}$ \\
\hline Network & Network & hsa05210 & Colorectal cancer & $5 / 14$ & $62 / 5894$ & 0 & $\begin{array}{c}37257040 \\
40885962932\end{array}$ \\
\hline Network & Network & hsa05212 & Pancreatic cancer & $4 / 14$ & $70 / 5894$ & 0 & $\begin{array}{c}67747040 \\
4088598\end{array}$ \\
\hline Network & Network & hsa05220 & Chronic myeloid leukemia & $4 / 14$ & $73 / 5894$ & 0 & $\begin{array}{c}67767040 \\
4088598\end{array}$ \\
\hline Network & Network & hsa04110 & Cell cycle & $4 / 14$ & $128 / 5894$ & 0.002 & $\begin{array}{l}70404088 \\
65022932\end{array}$ \\
\hline Network & Network & hsa05145 & Toxoplasmosis & $4 / 14$ & $133 / 5894$ & 0.002 & $\begin{array}{c}67747040598 \\
596\end{array}$ \\
\hline Network & Network & hsa05222 & Small cell lung cancer & $3 / 14$ & $85 / 5894$ & 0.006 & 5985966502 \\
\hline Network & Network & hsa04510 & Focal adhesion & $4 / 14$ & $200 / 5894$ & 0.006 & $\begin{array}{c}37255499596 \\
2932\end{array}$ \\
\hline Network & Network & hsa04012 & ErbB signaling pathway & $3 / 14$ & $87 / 5894$ & 0.006 & $\begin{array}{l}67763725 \\
2932\end{array}$ \\
\hline Network & Network & hsa05142 & $\begin{array}{l}\text { Chagas disease (American } \\
\text { trypanosomiasis) }\end{array}$ & $3 / 14$ & $104 / 5894$ & 0.009 & $\begin{array}{l}37257040 \\
4088\end{array}$ \\
\hline Network & Network & hsa04722 & $\begin{array}{l}\text { Neurotrophin signaling } \\
\text { pathway }\end{array}$ & $3 / 14$ & $127 / 5894$ & 0.015 & 37255962932 \\
\hline Network & Network & hsa05160 & Hepatitis C & $3 / 14$ & $135 / 5894$ & 0.017 & $\begin{array}{c}67743661 \\
2932\end{array}$ \\
\hline Network & Network & hsa04310 & Wnt signaling pathway & $3 / 14$ & $151 / 5894$ & 0.021 & $\begin{array}{l}37254088 \\
2932\end{array}$ \\
\hline Network & Network & hsa04630 & $\begin{array}{l}\text { Jak-STAT signaling } \\
\text { pathway }\end{array}$ & $3 / 14$ & $155 / 5894$ & 0.021 & 67766774598 \\
\hline Network & Network & hsa05014 & $\begin{array}{l}\text { Amyotrophic lateral } \\
\text { sclerosis (ALS) }\end{array}$ & $2 / 14$ & $54 / 5894$ & 0.026 & 598596 \\
\hline Network & Network & hsa05221 & Acute myeloid leukemia & $2 / 14$ & $58 / 5894$ & 0.028 & 67766774 \\
\hline
\end{tabular}




\begin{tabular}{|c|c|c|c|c|c|c|c|}
\hline Cluster & group & ID & Description & GeneRatio & BgRatio & p.adjust & geneID \\
\hline Network & Network & hsa04062 & $\begin{array}{c}\text { Chemokine signaling } \\
\text { pathway }\end{array}$ & $3 / 14$ & $189 / 5894$ & 0.03 & $\begin{array}{c}67742931 \\
2932\end{array}$ \\
\hline Network & Network & hsa05211 & Renal cell carcinoma & $2 / 14$ & $70 / 5894$ & 0.036 & 37257040 \\
\hline Network & Network & hsa05140 & Leishmaniasis & $2 / 14$ & $73 / 5894$ & 0.037 & 37257040 \\
\hline Network & Network & hsa04662 & $\begin{array}{l}\text { B cell receptor signaling } \\
\text { pathway }\end{array}$ & $2 / 14$ & $75 / 5894$ & 0.037 & 37252932 \\
\hline Network & Network & hsa04350 & $\begin{array}{l}\text { TGF-beta signaling } \\
\text { pathway }\end{array}$ & $2 / 14$ & $85 / 5894$ & 0.044 & 70404088 \\
\hline Network & Network & hsa04210 & Apoptosis & $2 / 14$ & $89 / 5894$ & 0.044 & 598596 \\
\hline Network & Network & hsa05215 & Prostate cancer & $2 / 14$ & $89 / 5894$ & 0.044 & 5962932 \\
\hline Network & Network & hsa05323 & Rheumatoid arthritis & $2 / 14$ & $92 / 5894$ & 0.045 & 37257040 \\
\hline
\end{tabular}

\subsection{GO LOCATION}

\begin{tabular}{|c|c|c|c|c|c|}
\hline Cluster & group & ID & Description & GeneRatio & geneID \\
\hline FullSet & FullSet & GO:0016020 & membrane & $75 / 99$ & $\begin{array}{c}23256570110652681191452925217514453945133178 \\
5927233939030835103323838644154785995973840 \\
85759287113355129018031049257256343023181 \\
3329927623521364648361288668665866587293832 \\
570751429484126986614346911653408050408050 \\
2280414091467917791779177917791779177917819 \\
2597811684181926470547057900181887622410540 \\
1655191585764499550\end{array}$ \\
\hline FullSet & FullSet & GO:0005576 & extracellular region & $53 / 99$ & $\begin{array}{c}57011134410875317859272308351033264415478928 \\
71132901803572563430231813337332933133646 \\
86681288665707514292698646914140914679177917 \\
791779177917791779172356423564235642356423564 \\
23564235642597871777105683555066224105401655 \\
19159550\end{array}$ \\
\hline FullSet & FullSet & GO:0005623 & cell & $113 / 99$ & $\begin{array}{c}68923256570111344106526811511879145292521 \\
108757514453945133178592723393903083510332 \\
3838644154785995973840857592871133551290 \\
85437180310492572563430231812209885033373329 \\
3313927623521364686684836128866866586658729 \\
62413832570767298495551429484126986876110146 \\
990861434691165323429234294080504080502280 \\
936041401046511123914679177917791779177917 \\
7917791723564235642356423564235642356423564 \\
81925978116841236097105683819265204574094705 \\
47057900181887555066224112007013105401655 \\
1197101915857732964499550\end{array}$ \\
\hline FullSet & FullSet & GO:0009295 & nucleoid & $1 / 99$ & 3313 \\
\hline FullSet & FullSet & GO:0030054 & cell junction & $12 / 99$ & $\begin{array}{c}547892818036343023313235212698610146614381926 \\
857\end{array}$ \\
\hline FullSet & FullSet & GO:0031974 & $\begin{array}{l}\text { membrane-enclosed } \\
\text { lumen }\end{array}$ & $53 / 99$ & $\begin{array}{c}5701511879145210875751431783838547838408575 \\
85437104925725302318122098850333733293313 \\
2352136468729570767298495548414691165323429 \\
234299360104657917791779177917791779177917710 \\
56835740947054705818875550662241120070131655 \\
19157329\end{array}$ \\
\hline
\end{tabular}




\begin{tabular}{|c|c|c|c|c|c|}
\hline Cluster & group & ID & Description & GeneRatio & geneID \\
\hline FullSet & FullSet & GO:0032991 & $\begin{array}{l}\text { protein-containing } \\
\text { complex }\end{array}$ & $70 / 99$ & $\begin{array}{c}57011065268115118792521108757514453945133178 \\
644154785998575355185437104926343023181220988 \\
3329927623521364686681288668665866538325707 \\
6729484126986876110146990861434691165323429 \\
234292280104659146791779177917791779177917 \\
7917259781168417681568352044705470581888755506 \\
622470131054016551915857732964499550\end{array}$ \\
\hline FullSet & FullSet & GO:0043226 & organelle & $104 / 99$ & $\begin{array}{c}68923256570111344106526811511879145292521 \\
10875751445394513317859272383864415478599597 \\
3840857592871133551290854371803104925725634 \\
30231812209885033373329331392762352136468668 \\
483612886687293832570767298495551429484126986 \\
8761101466143469116532342923429408050408050 \\
228093604140104659146791779177917791779177917 \\
791723564235642356423564235642356423564819 \\
259781168417105683819265204574094705470579001 \\
818875550662241120070131054016551197101915857 \\
732964499550\end{array}$ \\
\hline FullSet & FullSet & GO:0044215 & other organism & $3 / 99$ & 75143083510332 \\
\hline FullSet & FullSet & GO:0044217 & other organism part & $3 / 99$ & 75143083510332 \\
\hline FullSet & FullSet & GO:0044421 & $\begin{array}{l}\text { extracellular region } \\
\text { part }\end{array}$ & $49 / 99$ & $\begin{array}{c}113441087531785927264415478928711329018035725 \\
634302318133373329331336468668128866651429 \\
269864691414091467917791779177917791779177917 \\
23564235642356423564235642356423564259787177 \\
710568355506622410540165519159550\end{array}$ \\
\hline FullSet & FullSet & GO:0044422 & organelle part & $92 / 99$ & $\begin{array}{c}2325657011134410652681151187914529252110875 \\
751445394513317838386441547859959738408575 \\
928290854371803104925725634302318122098850 \\
3337332933139276235213646128866872938325707 \\
672984955514294841269866143469116532342923429 \\
408050408050228093601046591467917791779177917 \\
791779177917235642356423564235642356423564 \\
23564259781168417105683819265740947054705 \\
79001818875550662241120070131054016551915857 \\
732964499550\end{array}$ \\
\hline FullSet & FullSet & GO:0044425 & membrane part & $39 / 99$ & $\begin{array}{r}232561065268119145275144539451359272339390 \\
308351033238386441928711335512901803634302 \\
332992764836128866514294080504080502280819 \\
25978116841819264705470579001191585764499550\end{array}$ \\
\hline FullSet & FullSet & GO:0044456 & synapse part & $8 / 99$ & 57013838220988333725978116841819266449 \\
\hline FullSet & FullSet & GO:0044464 & cell part & $113 / 99$ & $\begin{array}{c}68923256570111344106526811511879145292521 \\
108757514453945133178592723393903083510332 \\
3838644154785995973840857592871133551290 \\
85437180310492572563430231812209885033373329 \\
3313927623521364686684836128866866586658729 \\
62413832570767298495551429484126986876110146 \\
990861434691165323429234294080504080502280 \\
936041401046511123914679177917791779177917 \\
7917791723564235642356423564235642356423564 \\
81925978116841236097105683819265204574094705 \\
47057900181887555066224112007013105401655 \\
1197101915857732964499550\end{array}$ \\
\hline FullSet & FullSet & GO:0045202 & synapse & $11 / 99$ & $\begin{array}{c}570138382209883337614325978116841819266224 \\
73296449\end{array}$ \\
\hline FullSet & FullSet & GO:0099080 & $\begin{array}{l}\text { supramolecular } \\
\text { complex }\end{array}$ & $5 / 99$ & 11344925213832228010540 \\
\hline Network & Network & GO:0016020 & membrane & $12 / 20$ & $\begin{array}{c}383752456774549970404088598417059629324869 \\
142\end{array}$ \\
\hline Network & Network & GO:0005576 & extracellular region & $4 / 20$ & 3837524554997040 \\
\hline
\end{tabular}




\begin{tabular}{|c|c|c|c|c|c|}
\hline Cluster & group & ID & Description & GeneRatio & geneID \\
\hline Network & Network & GO:0005623 & cell & $20 / 20$ & $\begin{array}{r}794238376776524537256774549970404088228093661 \\
598417059665024666293129324869142\end{array}$ \\
\hline Network & Network & GO:0030054 & cell junction & $3 / 20$ & 54995984869 \\
\hline Network & Network & GO:0031974 & $\begin{array}{l}\text { membrane-enclosed } \\
\text { lumen }\end{array}$ & $17 / 20$ & $\begin{array}{c}38376776524537256774549970404088228093661598 \\
4170596650229324869142\end{array}$ \\
\hline Network & Network & GO:0032991 & $\begin{array}{l}\text { protein-containing } \\
\text { complex }\end{array}$ & $15 / 20$ & $\begin{array}{c}3837372567745499408822809598417059665024666 \\
293129324869142\end{array}$ \\
\hline Network & Network & GO:0043226 & organelle & $20 / 20$ & $\begin{array}{c}794238376776524537256774549970404088228093661 \\
598417059665024666293129324869142\end{array}$ \\
\hline Network & Network & GO:0044215 & other organism & $1 / 20$ & 3837 \\
\hline Network & Network & GO:0044217 & other organism part & $1 / 20$ & 3837 \\
\hline Network & Network & GO:0044421 & $\begin{array}{l}\text { extracellular region } \\
\text { part }\end{array}$ & $4 / 20$ & 3837524554997040 \\
\hline Network & Network & GO:0044422 & organelle part & $17 / 20$ & $\begin{array}{c}38376776524537256774549970404088228093661598 \\
4170596650229324869142\end{array}$ \\
\hline Network & Network & GO:0044425 & membrane part & $4 / 20$ & 52455984170596 \\
\hline Network & Network & GO:0044456 & synapse part & $6 / 20$ & 52456774549959829312932 \\
\hline Network & Network & GO:0044464 & cell part & $20 / 20$ & $\begin{array}{c}794238376776524537256774549970404088228093661 \\
598417059665024666293129324869142\end{array}$ \\
\hline Network & Network & GO:0045202 & synapse & $6 / 20$ & 52456774549959829312932 \\
\hline
\end{tabular}

